


\section{Robot-ASSISTED BIOPSIES ON MR-DETECTED LESIONS}

Marcel K. Welleweerd 



\title{
RoBOT-ASSISTED BIOPSIES ON MR-DETECTED LESIONS
}

\section{DISSERTATION}

\author{
to obtain \\ the degree of doctor at the University of Twente, \\ on the authority of the rector magnificus, \\ prof. dr. ir. A. Veldkamp, \\ on account of the decision of the Doctorate Board, \\ to be publicly defended \\ on Friday, the $21^{\text {st }}$ of January, 2022, at $14: 45$
}

Marcel Klaas Welleweerd born on the $6^{\text {th }}$ of November, 1991 in Hardenberg, The Netherlands 
This dissertation has been approved by:

Prof. dr. ir. Stefano Stramigioli

Dr. Françoise J. Siepel

\author{
Promotor \\ Co-promotor
}

Cover design: Marcel K. Welleweerd

Printed by: Ridderprint

ISBN: $978-90-365-5322-3$

DOI: $10.3990 / 1.9789036553223$

Copyright ( 2021 Marcel K. Welleweerd, The Netherlands.

All rights reserved. No parts of this thesis may be reproduced, stored in a retrieval system or transmitted in any form or by any means without permission of the author. Alle rechten voorbehouden. Niets uit deze uitgave mag worden vermenigvuldigd, in enige vorm of op enige wijze, zonder voorafgaande schriftelijke toestemming van de auteur. 


\section{Graduation Committee:}

\section{Chair:}

Prof. dr. J. N. de Kok

University of Twente

Promotor:

Prof. dr. ir. Stefano Stramigioli

University of Twente

Co-promotor:

Dr. Francoise J. Siepel

University of Twente

Committee Members:

Prof. dr. ir. D.M. Brouwer PDEng University of Twente

Prof. dr. J. Dankelman TU Delft

Prof. dr. P. Fiorini University of Verona

Prof. dr. ir. C.L. de Korte University of Twente

RMM omans

ROBOTICS
ANECHATRONICS

\section{MURER}

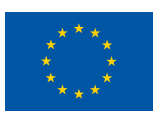

UNIVERSITY OF TWENTE.
This research was conducted at the Robotics and Mechatronics group of the faculty of Electrical Engineering, Mathematics and Computer Science of the University of Twente.

The work is part of the MRI and Ultrasound Robot-Assisted Biopsy (MURAB) project. The consortium consists of the following partners: University of Twente, Radboud UMC, University of Verona, KUKA, Siemens and the Medical University of Vienna.

The MURAB project has received funding from the European Union's Horizon 2020 research and innovation programme under grant agreement No. 688188.

Publisher:

University of Twente

Drienerlolaan 5

P.O. Box 217, 7500 AE, Enschede, The Netherlands 



\section{Contents}

\begin{tabular}{lll}
\hline & Introduction & 1
\end{tabular}

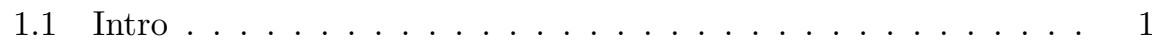

1.2 Breast cancer . . . . . . . . . . . . . . . . . . . 3

1.3 Related research . . . . . . . . . . . . . . . . . . . . 8

1.4 Thesis goal and outline. . . . . . . . . . . . . . . . . . 13

2 Design of an end-effector for robot-assisted ultrasound-guided breast biopsies 17

2.1 Introduction $\ldots \ldots \ldots \ldots \ldots \ldots$

2.2 Design Analysis $\ldots \ldots \ldots \ldots \ldots$

2.3 End-effector . . . . . . . . . . . . . . . . . . . . . 22

2.4 Experimental Validation . . . . . . . . . . . . . . . . 26

2.5 Discussion . . . . . . . . . . . . . . . . . . . 31

2.6 Conclusion and Recommendations $\ldots \ldots \ldots \ldots$. . . . . . 33

3 Combining Geometric Workspace Compliance with Energybased Joint Limit Avoidance 35

$3.1 \quad$ Introduction . . . . . . . . . . . . . . . . . . . . . 37

$3.2 \quad$ Joint limit avoidance with joint space potential energy $\ldots \ldots$. . . 39

3.3 Experimental validation $\ldots \ldots \ldots \ldots \ldots \ldots \ldots$

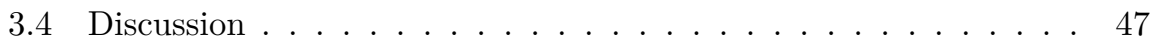

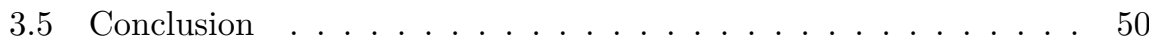

4 Automated robotic breast ultrasound acquisition using ultrasound feedback 51

4.1 Introduction . . . . . . . . . . . . . . . . . . 53

4.2 Automated robotic breast ultrasound . . . . . . . . . . . 55

4.3 Experimental validation . . . . . . . . . . . . . . . . . . 61

4.4 Discussion $\ldots \ldots \ldots \ldots \ldots \ldots \ldots \ldots$

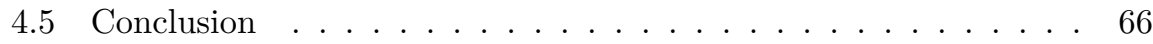


5 Out-of-plane corrections for autonomous robotic breast ultra$\begin{array}{lr}\text { sound acquisitions } & 67\end{array}$

5.1 Introduction . . . . . . . . . . . . . . . . . . . . . 69

5.2 The scanning algorithm . . . . . . . . . . . . . . . . 71

5.3 Experimental validation . . . . . . . . . . . . . . . 76

5.4 Discussion . . . . . . . . . . . . . . . . . . . . . . 80

5.5 Conclusion . . . . . . . . . . . . . . . . . . 82

6 Robot-assisted ultrasound-guided biopsy on MR-detected breast $\begin{array}{lc}\text { lesions } & 83\end{array}$

6.1 Introduction . . . . . . . . . . . . . . . . . . 85

6.2 Robot-assisted US-guided biopsy . . . . . . . . . . . . . . . . 87

6.3 Experimental validation . . . . . . . . . . . . . . . . . . . . . . . . . . . . . . . .

6.4 Discussion . . . . . . . . . . . . . . . . . 96

6.5 Conclusion . . . . . . . . . . . . . . . . . . . . . 98

7 MR Safe RGB Spectrophotometer-based Single Fiber Position $\begin{array}{lc}\text { Sensor } & 99\end{array}$

7.1 Introduction . . . . . . . . . . . . . . . . . . . . . . . . 101

7.2 Theory . . . . . . . . . . . . . . . . . . . . 103

7.3 Methods . . . . . . . . . . . . . . . . . . . . . 107

7.4 Results. . . . . . . . . . . . . . . . . . . . . . . 109

7.5 Discussion . . . . . . . . . . . . . . . . . . . . . . 112

7.6 Conclusion . . . . . . . . . . . . . . . . . . . . . . . . . . 114

7.7 Acknowledgements . . . . . . . . . . . . . . . . . . 114

$\begin{array}{llr}8 & \text { General discussion } & \mathbf{1 1 7}\end{array}$

8.1 Robotic setup . . . . . . . . . . . . . . . . . . 117

8.2 Future perspective . . . . . . . . . . . . . . . . . 121

$\begin{array}{lll}9 & \text { Conclusion } & 125\end{array}$

\begin{tabular}{lr}
\hline Summary & 127
\end{tabular}

\begin{tabular}{lr}
\hline Samenvatting & 129
\end{tabular}

\begin{tabular}{ll}
\hline List of publications & 131
\end{tabular}

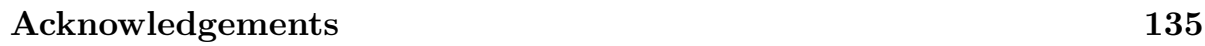

\begin{tabular}{|cc}
\hline Appendices & 139
\end{tabular} 
A Performance and application of a simple automated Magnetic Optical Density meter for analysis of Magnetotactic Bacteria 141

A.1 Introduction . . . . . . . . . . . . . . . . . . 143

A.2 Theory . . . . . . . . . . . . . . . . . . . . . . . 145

A.3 Method . . . . . . . . . . . . . . . . . . . . . 150

A.4 Results. . . . . . . . . . . . . . . . . . . . . . . . . . . 159

A.5 Discussion . . . . . . . . . . . . . . . . . . . . . 173

A.6 Conclusion . . . . . . . . . . . . . . . . . . . . . . 178

A.7 $C_{\mathrm{mag}}^{*}$ and $C_{\mathrm{mag}}$ approximations $\ldots \ldots \ldots \ldots \ldots \ldots \ldots$

A.8 Cotangens approximation $\ldots \ldots \ldots \ldots$. . . . . . . . . . 180

A.9 Measurements . . . . . . . . . . . . . . . . . . . . . . . . . 181

\begin{tabular}{ll}
\hline References & 181
\end{tabular} 



\section{General introduction}

\subsection{Intro}

Robots are on the rise, but not in the typical sci-fi sense. Next to being essential figures in movies, robots play an ever more important role in our daily lives. Early predictions stated that robotics would mainly be applied in industrial applications, whereas applications outside factories would only make up to $1 \%$ of global sales 1]. However, these were quite wrong: service robotics — robots or equipment with a degree of autonomy that perform practical tasks for humans, excluding industrial automation applications - make up around $10 \%$ of the annual global robotics turnover in 2020 [2, 3]. An increasing number of people own a service robot such as an automatic vacuum cleaner or a lawnmower. A glance through the 'Robots at work' section of the Handbook of Robotics [1] teaches us that the list of possible applications is extensive, ranging from industrial and space applications to the entertainment industry. Table 1.1 provides an overview of application areas.

This increase in interest is similar in the medical world; the sales in this area accounted for $47 \%$ of the professional service robot turnover in 2019 [3. Medical or interventional robots can be defined as surgical tools that (semi-)autonomously aid the surgeon in (minimally-)invasive medical procedures. Often, medical practitioners take a critical stance towards introducing interventional robotics. They may feel that they will get 'replaced' since traditionally, robots are applied in areas where they can take over specific repetitive tasks from a human, such as vacuum cleaning or harvesting fruit. However, just like in other application areas, medical robots should provide significant advantages to be accepted and widely deployed. For interventional robots, these advantages are often achieved by synergistic cooperation between the human and the robotic device. Table 1.2 shows an overview of several of the strengths and weaknesses of both humans and robots.

From Table 1.2 it is clear that the strong suit of humans is their flexibility, their ability to act upon unexpected or new information sources, and their versatility. In contrast, robots have superior end-effectors for a specific task, are geometrically accurate, more stable and able to process large amounts of data. 
Teleoperated robots are very suitable for achieving synergy since the robot's motions are instructed by the surgeon, who is responsible for decision-making and able to adapt to unforeseen situations. The surgeon's movements appear scaled and filtered at the robotic end-effector, and thus increased precision is achieved. Additionally, this approach may improve safety since invisible boundaries where the tool will not go can be created, and online support for the procedure may be supplied. The best-known and maybe even the most successful robot to date is a teleoperated robot: the Da Vinci surgical system 17.

Next to teleoperated robots, robots with various degrees of autonomy have been introduced, which can be categorized according to multiple schemes 18 20. On the one side, there are 'passive' robots without autonomy that passively assist the surgeon by holding a tool or constraining the surgeon's movements. Passive robots were mainly introduced in light of safety considerations in the medical environment. An example is a passive robotic arm that dynamically implements constraints to prevent the surgeon from moving away from the pre-planned trajectory 21]. On the other side, there are robots that autonomously perform

Table 1.1: Fields in which robotics are being applied along with concrete examples.

\begin{tabular}{|c|c|c|}
\hline Application & Example & Cit. \\
\hline Industrial & Robotic car assemblage at the Tesla gigafactory & 4 \\
\hline Space & Robots such as the Mars Rover for space exploration & 5 \\
\hline $\begin{array}{l}\text { Agriculture and } \\
\text { forestry }\end{array}$ & The SWEEPER sweet pepper harvester & 6 \\
\hline Construction & Bots2Rec, a system proposed for robotic asbestos removal & 7 \\
\hline $\begin{array}{l}\text { Hazardous } \\
\text { applications }\end{array}$ & Gamma-ray imaging after the Fukushima Daiichi disaster & 8 \\
\hline Mining & $\begin{array}{l}\text { UNEXMIN was a European project focused on the } \\
\text { exploration of flooded mines for their mineral potential }\end{array}$ & 9 \\
\hline Disaster & $\begin{array}{l}\text { The SHERPA project was a project for robot-assisted alpine } \\
\text { rescue operations }\end{array}$ & 10 \\
\hline $\begin{array}{l}\text { Surveillance and } \\
\text { security }\end{array}$ & $\begin{array}{l}\text { The cannachopper; A helicopter used experimentally by the } \\
\text { Dutch police to find marijuana nurseries }\end{array}$ & 11 \\
\hline Intelligent vehicles & $\begin{array}{l}\text { The self-driving cars of Waymo, formerly known as the } \\
\text { Google self-driving car program }\end{array}$ & 12 \\
\hline Domestic & Automatic vacuum cleaners, such as the Roomba of IRobot & 13 \\
\hline $\begin{array}{l}\text { Rehabilitation and } \\
\text { health care }\end{array}$ & Exoskeletons which support older people during walking & 14 \\
\hline Medical robotics & The Da Vinci surgical system for minimally invasive surgery & 15 \\
\hline $\begin{array}{l}\text { Entertainment } \\
\text { industry }\end{array}$ & Robotic stunt doubles in movies & 16 \\
\hline
\end{tabular}


a certain task in a procedure. An example of such a robot is the one drilling an access hole for a cochlear implant 22 .

Medical robotics has now successfully been integrated into a range of medical procedures. These include, among others, various endoscopic procedures, orthopedics, radiotherapy, neurosurgical interventions and biopsies $[20,23,24]$. However, the introduction of robots to the medical world is just starting, and continuous technological advancements will lead to the integration of interventional robots in even more areas 20 . This thesis looks into applying medical robotics to breast cancer screening and diagnosis. To understand why this application is clinically relevant, first some background information regarding breast cancer, the current diagnostic workup, and its treatment is provided. After that, an analysis is given of the probable beneficial role of robotics in this process. This chapter finalizes with the research question and the thesis outline.

\subsection{Breast cancer}

Breast cancer affects about one in eight women worldwide. In women, it is the most common cancer, and it is the leading cause of cancer death in many countries 26]. This means breast cancer is a considerable burden for public health care, the economy, and not in the last place: the affected women themselves.

Table 1.2: Strengths and limitations of both humans and robots 25].

\begin{tabular}{|c|c|c|}
\hline Entity & Strengths & Limitations \\
\hline$\overline{\text { Human }}$ & $\begin{array}{l}\text { - Excellent hand-eye coordination } \\
\text { - Excellent dexterity } \\
\text { - Able to integrate and act on multiple } \\
\text { information sources } \\
\text { - Easily trained for multiple tasks } \\
\text { - Versatile and able to improvise }\end{array}$ & $\begin{array}{l}\text { - Tremor limits fine motion } \\
\text { - Limited manipulation ability and } \\
\text { dexterity outside natural scale } \\
\text { - Cannot see through tissue } \\
\text { - Bulky end-effectors (hands) } \\
\text { - Hard to keep sterile } \\
\text { - Affected by radiation and airborne } \\
\text { infections }\end{array}$ \\
\hline Robot & $\begin{array}{l}\text { - Untiring and stable } \\
\text { - Immune to radiation and airborne } \\
\text { infections } \\
\text { - Can be designed to operate at } \\
\text { different scales of motion and payload } \\
\text { - Able to integrate multiple sources of } \\
\text { numerical and sensor data } \\
\text { - Ruling out 'human errors' }\end{array}$ & $\begin{array}{l}\text { - Poor judgment } \\
\text { - Hard to adapt to new situations } \\
\text { - Limited dexterity } \\
\text { - Limited hand-eye coordination } \\
\text { - Limited haptic sensing } \\
\text { - Limited ability to integrate and } \\
\text { interpret complex information }\end{array}$ \\
\hline
\end{tabular}




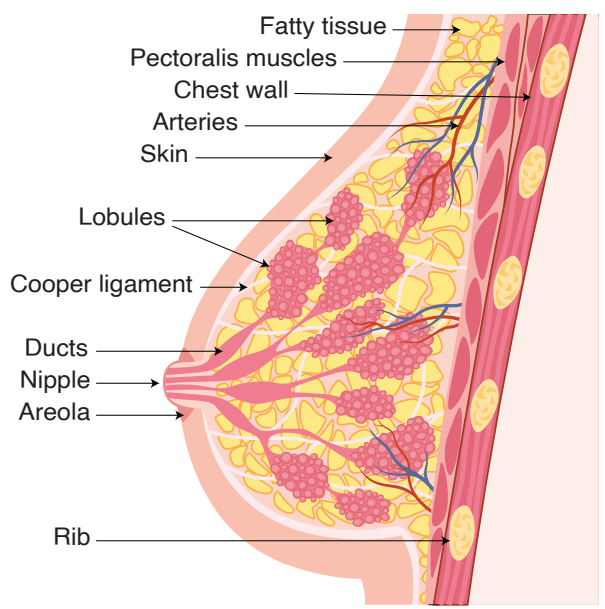

Figure 1.1: Anatomy of the human breast. Arrows indicate the various tissue types.

\subsubsection{Pathology}

Figure 1.1 gives an overview of the female breast's anatomy. The breast lays on top of the pectoralis muscles. All structures in the breast which are not fatty tissue are denominated fibroglandular tissue. The ratio between fibroglandular and fatty tissue varies per individual and with age. A dense breast contains a relatively high amount of fibroglandular tissue.

Cancer often forms in the ducts or the lobes. Carcinogenesis mostly takes place in the epithelial cells, which line the outer surface of the tissue. The cancer stage progresses to an in situ carcinoma after abnormal proliferation started. An in situ carcinoma has not yet spread beyond the location where it first formed and is also regarded as non-invasive cancer. This type of cancer is considered a precursor for invasive carcinomas, where cancer has spread beyond the layer of tissue in which it originally developed. Non-invasive tumors in the ducts or lobes are referred to as ductal carcinoma in-situ (DCIS) or lobular neoplasms, respectively. If cancer has progressed to the invasive stage, tumors will become an invasive ductal carcinoma (IDC) or an invasive lobular carcinoma (ILC). However, for most invasive tumor types, the term invasive carcinoma of no special type (NST) is utilized because the origin of the cancer is unproven. There are many more types of non-invasive and invasive cancers, of which an overview can be found in for instance 27].

Some women have a higher chance of developing cancer than others. Nonhereditary factors play a significant role in breast cancer incidence [28]. However, also genetic predispositions such as the breast cancer (BRCA) 1 and BRCA2 gene mutation and having relatively dense breasts put women at increased risk 29 . 


\subsubsection{Treatment}

There are three crucial steps in treating breast cancer: detection, diagnosis, and treatment.

\section{Detection}

Breast cancer is detected through self-examination, screening programs or incidental findings. Since the survival rate of breast cancer is highly dependent on early detection of the disease, many countries have screening programs in place which routinely image asymptomatic women to assess whether cancer is present [30]. Many imaging techniques are available for screening, the most common ones being mammography, ultrasound and magnetic resonance imaging (MRI).

Mammography - an x-ray of the breast — is the standard for screening and detecting breast cancer. It has been extensively studied and is proven to reduce the mortality rate. However, it has limitations in selectivity and sensitivity: no distinction can be made between solid and cystic masses, its sensitivity is reduced in dense breasts, and it misses approximately $10-15 \%$ of cancers 29,31 .

In contrast to mammography, ultrasound is a cross-sectional technique, which displays the tissue without overlap. Therefore, supplementing mammography with ultrasound can significantly increase the sensitivity of the screening process, especially for dense-breasted women. However, detecting non-palpable masses with a hand-held imaging device is highly operator-dependent and can take much time. Utilizing an automated breast ultrasound device can partly overcome these disadvantages 32 .

MRI is considered the most sensitive imaging modality for the early detection of breast cancer $[29,33$. The utilization of MRI is mainly considered for high-risk patients because its usage is very costly. High-risk patients, for example, women with a proven gene mutation or a family history of breast cancer, benefit the most because the tumor generally develops faster. Also, this group demonstrates a higher rate of interval cancers with the annual mammography screening alone [34].

\section{Diagnosis}

Follow-up diagnostic research can confirm the nature of an abnormality. Although steps are made in the diagnostic accuracy of breast imaging, breast cancer diagnosis is often supplemented by histopathological assessment of tissue acquired through biopsy [35, 36]. A biopsy is a process in which a sample is taken from the lesion to confirm malignancy via histopathological analysis. During an imageguided percutaneous biopsy, a specialized needle is directed to the suspicious site under local anesthesia. Three types of needles are utilized: core, vacuumassisted, and fine needles - the latter is increasingly replaced by the former two alternatives. Commonly used imaging modalities to guide the procedure are mammography, ultrasound and MRI [37]. 


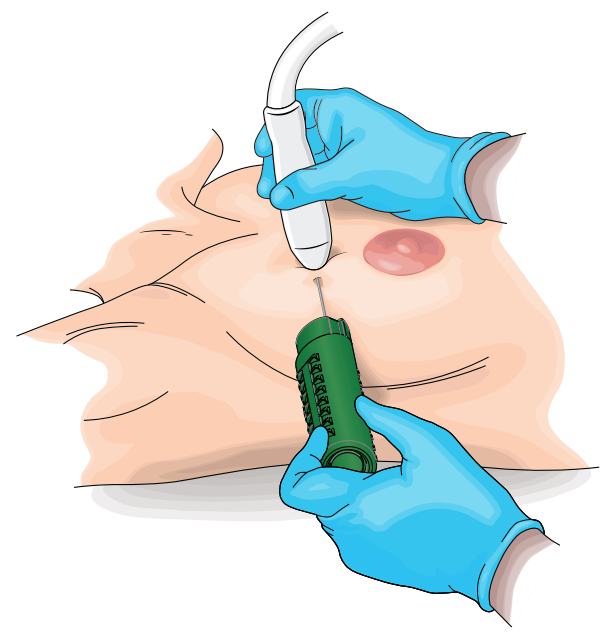

Figure 1.2: An impression of the ultrasound-guided biopsy. The left hand carries a core needle biopsy device, the right one holds the ultrasound probe.

Mammographic guidance is utilized for lesions detected on mammography that are not visible on ultrasound. The lesion position is often estimated via stereotaxis, where two oblique projections are combined to estimate the lesion position. Hence, this type of biopsy is termed a stereotactic biopsy. Stereotactic biopsies make up a significant portion of the biopsies performed in some hospitals [38. The traditional stereotactic biopsy takes around $30 \mathrm{~min}$ but the duration has been improved to 10-15 min with the introduction of tomosynthesis. This type of biopsy is mostly performed with a vacuum-assisted needle aimed by a dedicated device attached to the chair or table with the patient 37.

The ultrasound-guided biopsy is the most popular technique because of its realtime guidance, relatively good patient comfort, wide availability, short learning curve, and cost and time effectiveness (5-15 min) [37, 39]. The radiologist performs the ultrasound-guided biopsy by navigating the biopsy device with one hand and holding the ultrasound probe in the other. This approach is also termed the freehand technique and is depicted in Figure 1.2. This technique often uses a core needle, and radiologists can accurately perform biopsies on lesions down to $10 \mathrm{~mm}$ with a $16-18 \mathrm{G}$ needle diameter [40]. Several studies state that at least five tissue samples should be taken for a reliable result [41]. An ultrasound-guided biopsy can be performed on both lesions detected by mammography or MRI. However, the success rate of MRI-directed ultrasound-guided biopsies is limited, and they are not advised for lesions $<10 \mathrm{~mm}$ [42, 43]. The success rate is directly related to the visibility of the lesion on the ultrasound image, and the lesion may not be visible for several reasons: the woman's position is different during an ultrasound-guided biopsy (supine or supine oblique) compared to the initial MRI 
imaging (prone). The lesion's position and shape may change during repositioning. Additionally, the difference in image types - a cross-sectional image versus a three-dimensional image - and the difference in imaging techniques make finding the lesion difficult 44, 45. As a result, the procedure is time-consuming, operator-dependent and post-procedural imaging to confirm accurate sampling is preferred [42].

Although ultrasound-guided biopsies are preferred, approximately $43 \%$ of magnetic resonance (MR)-detected lesions are referred for an MRI-guided biopsy [45]. The lesion position is more readily identified since it was previously identified, and the patient's position is more similar to the initial breast MRI. During an MRI-guided biopsy, the breast is compressed between two plates, one fixed near the patient's sternum, the other mobile. The mobile plate contains a raster through which a plastic introducer sheath and the vacuum-assisted biopsy needle are navigated to the lesion. The first step is to scan the breast to identify the lesion. Then, software calculates the correct raster coordinates, after which the plastic sheath is inserted, and an MR sequence is performed to confirm proper placement. Then, the biopsy needle is introduced and the biopsy is taken. Also, a clip was deployed, which is used to verify correct sampling via a final MR sequence. Lesions with a $5-6 \mathrm{~mm}$ diameter are targeted accurately [46]. This type of biopsy has multiple disadvantages: it is more complex because there are more steps involved; there is no real-time feedback; there are time constraints due to the transient nature of the contrast enhancement; the space in the MRI is restricted, and no metallic objects are allowed; lesions located far anterior or far posterior are challenging to reach because of the one-sided access to the breast and limited freedom to configure the needle; the procedure is more painful since a larger diameter needle is utilized than during an ultrasound-guided biopsy, and very costly due to the various steps and time (sometimes exceeding $30 \mathrm{~min}$ ) involved [37]. Consequently, the procedure is also less comfortable for the patient 45].

\section{Treatment}

The treatment options for breast cancer vary from local treatment by tumor eradication or resection with radiotherapy or surgery to systemic therapy with endocrine or chemotherapy. With breast-conserving surgery, only the part of the breast that has cancer is removed. A comprehensive overview of treatment strategies is given in 47]. The histological assessment performed on the tissue sample captured by the biopsy procedure forms the basis for composing the treatment plan [48]. Thus, a reliable outcome of the biopsy procedure is of paramount importance: missed malignancies can result in mistreatment. 


\subsection{Related research}

The biopsy procedure is an essential phase in the diagnostic workup of breast cancer: incorrect needle placement may have far-reaching consequences since a false-negative would lead to delayed diagnosis and cancer treatment [49]. This thesis primarily focuses on ultrasound-guided and MRI-guided biopsies on MRdetected lesions. The previous section shows that the outcome and procedure of biopsies on MR-detected lesions could benefit from improved localization of the lesion and accuracy of the needle placement. The coming sections discuss recent advancements in the area of lesion localization and lesion targeting and the potential role of robotics herein.

\subsubsection{Lesion localization}

Currently, the MR-detected lesion is preferably sampled with an ultrasoundguided biopsy 45]. Therefore, much research is focused on supporting the spatial cognition of the radiologist. Spatial cognition is the ability to find the corresponding location of the lesion with the ultrasound probe based on previously acquired MRI data and is an influential factor in minimally invasive surgery [50]. The high deformity of the breast further complexifies adequate localization.

\section{Volumetric breast ultrasound}

One way to improve localization is to go from regular 2D ultrasound images to volumetric breast ultrasound scanning. Several studies show that lesions previously detected on MRI are more readily identified on volume ultrasound acquisitions of the breast $[51,53]$. The increased number of features with which the acquired data can be (mentally) registered with the MRI data helps the radiologist to find the lesion. Another advantage is that the number of MRI-guided biopsies is lower utilizing this technique [54]. However, the currently applied volumetric ultrasound acquisition methods merely play a role in deciding whether to proceed with an ultrasound-guided or an MRI-guided biopsy. Also, even though the imaging technology continuously improves, like with the introduction of ultrasound tomography, most commercially available volumetric ultrasound scanners suffer various limitations [55]. Examples of limitations include tissue deformation due to compression or buoyancy; limited flexibility, e.g., not being suitable for all breast sizes; limited field of view, resulting in gaps in the volume and the inability to evaluate axillary lymph nodes [56, 57]. Furthermore, they lack supplementary technologies such as Doppler and elastography [57. 


\section{MRI/ultrasound fusion}

Another available technology supporting the radiologist with the localization is MRI/ultrasound fusion - also termed co-registration or real-time virtual sonography. In this technology, the current ultrasound image contains a realtime overlay of the MRI data. Most systems use an electromagnetic tracker to measure the current pose of the ultrasound probe with respect to the patient. This technology improves the chance of sonographic-MRI correlation, and an additional advantage is that these lesions also qualify for an ultrasound-guided biopsy [58 62]. Since the biopsy is performed in supine position, an additional supine MRI scan is necessary to achieve accurate registration. This second scan is a significant disadvantage: it has lower image quality than the original prone MRI, is time-consuming and requires an additional contrast agent administration [63]. Even though the extra scan improves the registration, the manual registration procedure of the MR images with the ultrasound images is not that accurate, around $5 \mathrm{~mm}$ 64].

\section{Novel alternatives}

Novel alternatives include an automated cone-based volumetric ultrasound scanner that performs a high-resolution volumetric scan of the breast that is registered with preoperative MRI 65]. It overcomes some of the shortcomings of other systems, such as shadowed regions and missed sections of the breast due to the probe's orientation. A disadvantage of this system is the necessity of an MRI with the breast submerged in water to compensate for deformations due to buoyancy. Furthermore, photoacoustics is a promising diagnostic tool that measures ultrasound waves generated by thermoelastic expansion of the tissue caused by the absorption of a laser beam. It can measure functional parameters such as hemoglobin and oxygen concentration, and thus, the specificity may be enhanced compared to traditional imaging modalities [66].

\section{Robotic ultrasound acquisitions}

Robotic ultrasound acquisitions have the potential to excel in the areas where the other solutions fall short; accuracy of the registration of ultrasound with MRI, the flexibility of the scanner, field of view, deformations occurring during the scan and acquisition quality may all be improved.

Firstly, registration of the preoperative MRI with the ultrasound acquisitions is more readily performed since the robot may be equipped with a depth camera or stereo cameras to localize the patient based on surface scans or marker registration 67, 68].

Utilizing the same approach, also the scanner's flexibility may be improved; if a precise reconstruction of the skin surface is available, the robot can use this to generate a patient-specific trajectory. Additionally, the dexterity of a 
robotic manipulator allows for a greater variety of poses of the ultrasound probe, enabling the robot to scan a more extensive range of breast sizes and shapes, even including the axillary lymph nodes.

Also, deformations occurring during a scan are minimized by patient-specific trajectories since the probe follows the original surface and does not take a one-size-fits-all approach. Furthermore, sensory information can be used in a feedback loop to continuously update the preoperatively planned trajectory. This loop compensates for imperfections of the trajectory and involuntary patient movements. Previous research used both force and ultrasound image feedback. Force feedback was used to achieve acoustic coupling and to change the probe's pose and may be accomplished by direct measurement of a force sensor attached between the probe and the robot flange or by deriving the force from the torques measured by the joint sensors $69 \sqrt{74}$. Also, a variety of image features has been used to achieve both pose corrections of the probe and acoustic coupling. These include image intensity, image similarity, feature tracking, image moments, speckle tracking and confidence maps $69,73,75,79$. Some methods are more suitable to track a target, whereas others are also relevant for volume acquisitions.

Finally, the quality of ultrasound acquisitions can be improved because the robotic manipulator can produce evenly spaced slices due to precise joint sensors and coordinated motions. Additionally, the robot could use the $2 \mathrm{D}$ images of a standard ultrasound probe to build up the $3 \mathrm{D}$ volume. This type of probe is widely available and produces high-quality images. Due to the range of precise feedback mechanisms, robotic ultrasound scans are more reproducible than scans performed by a radiologist 80 .

Next to these improvements, using a regular ultrasound probe opens up new possibilities, such as performing elastography and utilizing the same probe for the biopsy.

\subsubsection{Needle placement}

The previous section presents research on supporting the radiologist's spatial cognition. While support on this aspect does improve the rate of ultrasoundguided biopsies on MR-detected lesions, the accuracy of the needle placement is still limited by the hand-eye coordination of the radiologist. Increased accuracy can decrease the number of false negatives, the minimal lesion size that is reliably sampled, the number of required samples, and the needle diameter. Also, the latter two are associated with reduced patient comfort and an increased risk of neoplastic seeding, which is tumor formation originating from displaced tumor cells during the procedure 81,82 . The following presents both unactuated and robotic solutions aimed to improve the accuracy of needle placement. 


\section{Unactuated solutions}

There are unactuated needle guides for ultrasound-guided, MRI-guided and stereotactic biopsies. These needle guides support the radiologist by limiting the needle motion to the insertion direction. The guide is locked in place to maintain the correct needle trajectory, and the radiologist controls the needle depth utilizing markings on the needle shaft. Software helps find the needle guide's correct configuration and the desired needle depth 83 85. Also, the previously discussed cone scanner for ultrasound breast volume acquisitions can perform ultrasound-guided biopsies similarly 86.

Although these systems can use image feedback to confirm correct needle placement, they cannot adjust the needle trajectory based on real-time feedback to compensate for deformations due to needle insertion or patient movements. Several guiding systems are available that preserve the freehand technique, as discussed in Section 1.2.2. These systems are essentially an add-on for the ultrasound probe and lock some degrees of freedom such that the needle manipulation is more consistent and always in the field of view of the probe 87,89. Some of these devices rely on locking the needle trajectory relative to the ultrasound probe. As the radiologist holds the probe, the desired needle trajectory relative to the probe constantly changes. Thus, locking the needle relative to the probe does not necessarily ensure accurate needle placement. The system presented by Suthakorn et al. [89. does not have this issue since optical tracking continuously provides the radiologist with an updated desired needle trajectory. The system has been shown to enhance hand-eye coordination, especially of inexperienced radiologists, and may allow MRI/ultrasound fusion.

\section{Robotic needle placement}

Robots do not need support with spatial cognition; robots are inherently good at processing large amounts of spatial data and coupling this to accurate physical action. Additionally, real-time sensor information may be fused with preoperative data or used to update the lesion position. These aspects make the biopsy procedure a very suitable procedure to be performed by robots. Robotic biopsies have already been performed on various organs such as bone, lung, brain or brainstem, prostate, and liver 90 94]; also, various research is conducted after robotic breast biopsy systems and approaches [95, 96. The following presents robotic ultrasound-guided needle insertion, deformation management, and robotic MRI-guided needle insertion approaches.

Some robotic ultrasound-guided biopsy systems do not leverage the real-time capabilities of ultrasound imaging. Both Megali et al. [97] and Kettenbach et al. [98. presented a system that performs a biopsy based on a preoperatively acquired position found with an optically tracked ultrasound probe. Other approaches utilize robotic needle placement in conjunction with robotic ultrasound probe manipulation. The degrees of freedom of the needle with respect to the ultrasound 
probe vary per approach. A setup comprised of two robots - one for needle insertion, the other for probe placement - achieves optimal flexibility in planning and monitoring the needle trajectory [99]. However, the extra robot introduces the necessity of an inter-robot calibration, which could be a source of errors. Other designs stay closer to the technique of the radiologist, as presented in Figure 1.2 [100. An advantage is that the needle is in the ultrasound plane by default, which is the standard feedback mechanism used by radiologists, and the design of the needle manipulator is relatively simple. Additionally, one robot can perform both ultrasound acquisitions and biopsies, which prevents the necessity of transferring the lesion coordinates between systems. This fixed configuration does exclude switching to alternative insertion methods in which the needle is perpendicular to the image plane, as presented by, e.g., Vrooijink et al. [101] and Abayazid et al. 74]. However, these methods are less common-place in breast cancer diagnostics. Generally, the accuracy of the presented robotic systems is well below the previously reported $10 \mathrm{~mm}$ of the radiologist, and their limit is around $1 \mathrm{~mm} 98,102,103$. Nevertheless, these systems were tested in highly simplified in-air, phantom, or ex vivo experiments and most studies assumed that the target is visible on the ultrasound image. This assumption may not be correct for MR-detected lesions.

The deformity and motions of the breast may cause the lesion to displace during the biopsy procedure 104. A robot does minimize unpredictable motion due to stable probe and needle handling, but the lesion position will change due to interaction forces and involuntary patient movements. Deformity and motions can either be limited or compensated for based on predictions or real-time adjustments of the needle trajectory during the procedure.

Generally, the patient is positioned in prone position to limit motions. In prone position, involuntary movements such as breathing have minimal impact on the breast, which is one reason why an MRI scan is taken this way 105. Additionally, the examined breast may be fixated to minimize deformation even further. Hatano et al. [103 achieved breast fixation with a specialized needle guide. Currently, ultrasound-guided biopsies in prone position are considered undesirable from an ergonomics point of view. Indeed, sonographers who regularly perform ultrasound scans and biopsies are prone to suffer from musculoskeletal disorders [63, 106]. On the other hand, robots do not suffer from fatigue and do not mind their position relative to the patient. Furthermore, performing the biopsy in prone position renders the additional supine MRI superfluous.

Deformations can be compensated for either preoperatively, using deformation modeling, or intraoperatively, using image processing on the ultrasound images. Deformation modeling allows the lesion position to be updated based on expected interactions of the probe and needle with the patient 107]. Image processing methods enable the robot to correct the lesion position and needle trajectory based on the current image input. Tracking methods can be based on segmentation of the lesion or general deformation measurements using optical 
flow 108, 109. Both the lesion and the needle can be tracked simultaneously 110. Needle tracking is helpful to compensate for needle deformations or support the system's forward kinematics. Observations may be used to update the needle trajectory or counteract deformations via an actuator placed on the breast surface 111 114.

Alternatively, some robots can operate inside the MRI bore and use the MRI as a sensor for the biopsy procedure. The advantage of this type of robot is that registration between the patient and the robot is less complex since both are visible on the same dataset. Additionally, there is no need to merge several data types, as with ultrasound/MRI fusion. However, this approach adds serious design constraints since no metallic objects are allowed in this location. Various designs have been presented utilizing a range of actuation techniques such as piezoelectric, pneumatic, hydraulic, and tendon-driven motors [115 118]. Another disadvantage of in-bore robots is the higher MR time.

\subsection{Thesis goal and outline}

The previous section explained that robotics might have an important role in the workup of breast cancer treatment, both in lesion localization and needle placement. In ultrasound acquisitions, the accuracy of ultrasound/MRI fusion, the field of view, tissue deformation, and acquisition quality may be improved. In needle placement, spatial cognition, the accuracy and deformation compensation may be improved, both inside and outside the MRI bore.

Currently, most systems are focused on ultrasound-detectable lesions. Consequently, assumptions are made in the system design, such as the visibility and segmentability of the lesion in ultrasound. For MR-detected lesions, these assumptions do not necessarily hold, and therefore, a different approach would be required. Thus, this thesis focuses on how robots could assist radiologists in performing biopsies on suspicious lesions previously detected on MRI. Two approaches are treated: ultrasound-guided and MRI-guided biopsies.

\subsubsection{Ultrasound-guided}

Primarily, this research is conducted in the MRI and Ultrasound Robot-Assisted Biopsy (MURAB) project. This project is about the clinical challenge of performing ultrasound-guided biopsies on MR-detected lesions. Figure 1.3 presents the proposed setup and the most critical steps of the workflow. The chapters of this thesis represent sections of the workflow, as the MURAB project is a European project with multiple partners who worked on different aspects. The following discusses the workflow, which role the content of the chapters plays, and mentions some co-authored work. 
Figure 1.3(a) shows how the patient lays on a dedicated bed in prone position with the examined breast through a hole. This setup is chosen such that deformations with respect to the previously obtained breast MRI and the impact of involuntary patient movements such as breathing are minimal. A seven-degrees-of-freedom robotic manipulator with a dedicated end-effector is placed underneath the patient. Chapter 2 introduces this end-effector, which is specifically designed for ultrasound-guided biopsies on MR-detected lesions. Compliant behavior of the robot is essential, while the robotic manipulator navigates in a complex environment with the patient, the radiologist and several nearby objects. Chapter 3 elaborates on a compliant control theory for redundant robotic manipulators. In this theory, the manipulator continuously optimizes its configuration for complex trajectories by moving away from the joint limits while simultaneously respecting the desired trajectory of the end-effector itself. Figure 1.3(b) shows how the robot determines the patient's position with respect to itself. In the final setup, this is performed by stereo cameras that detect colored multi-modality markers. These markers are detectable on camera, ultrasound images, and MRI 119]. The breast's outline with respect to the robot is determined with this localization step and by extracting the breast's shape from the preoperative MRI. Some details of this registration step are outlined in Chapter 2 also. Chapter 4 shows how the registration step and the preoperative images are exploited to plan a patient-specific trajectory for ultrasound acquisitions (Figure 1.3(c)). Additionally, this chapter shows how imperfections of this trajectory are compensated for using ultrasound feedback. Chapter 5 further elaborates on this topic and shows how scanning may be possible without patient specific information. Please refer to Nikolaev et al. [120] for more information on the acquired ultrasound volumes. Next, the acquired ultrasound volume is registered with the MRI based on the detected markers (Figure 1.3. d)) This step obtains the lesion position in robot coordinates. However, the lesion position will change upon contact of the ultrasound probe with the breast. A modeling step could compensate for this, as is discussed in (Figure 1.3.e) [121. Elastography could be performed to obtain the correct modeling parameters such as tissue stiffness. Elastography data can be acquired by utilizing an acoustically transparent pressure pad [122]. The following steps are correctly placing the ultrasound probe on the patient's skin with the lesion in the field of view, and guiding the needle to the correct location (Figure $1.3(\mathrm{f})$ and $(\mathrm{g})$ ). Chapter 6 discusses how tissue and needle deformations can be compensated for during the biopsy procedure.

\subsubsection{MRI-guided}

Alternatively, MR safe robots could perform biopsies on MR-detected lesions. Work presented at the Hamlyn Symposium shows how to build and control a pneumatically actuated robotic manipulator which operates inside the MRI 
bore [123]. Chapter 7 elaborates on an MR safe position sensor based on a spectrophotometer, which could be integrated into a robot that operates inside the MRI bore. As such, the robot precisely knows its joint positions, and as a result, the end-effector location. This work builds on knowledge acquired in the design of the magnetic optical density meter, of which you can find more detailed information in appendix A 


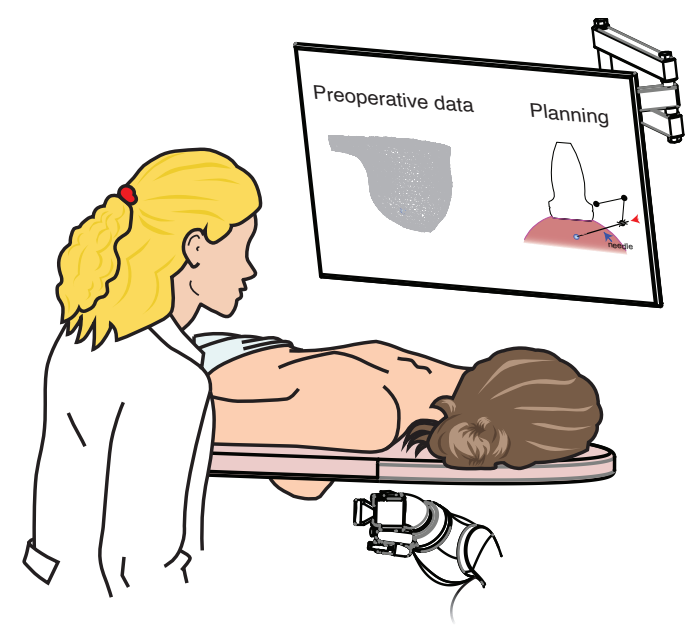

(a)

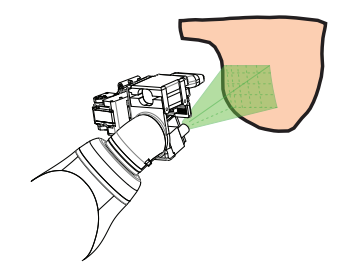

(b)

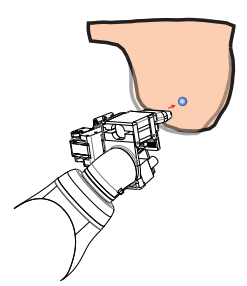

(e)

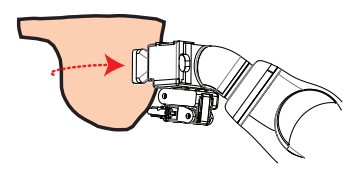

(c)

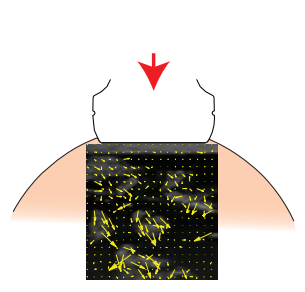

(f)

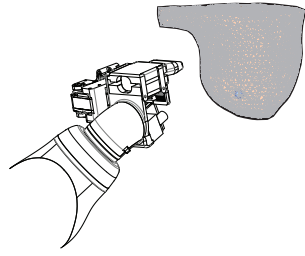

(d)

Figure 1.3: Workflow of a robot-assisted ultrasound-guided biopsy. (a) Initial planning. (b) Breast localization based on e.g. structured light projection. (c) Volumetric breast ultrasound acquisition. (d) The lesion position with respect to the robot is determined with ultrasound/MRI fusion. Deformations happen upon contact of the ultrasound probe with the skin. Modeling (e) and tracking (f) can be used to compensate for this effect. (g) The intervention takes place where the robot helps the radiologist aim the needle and compensates for deformations caused by needle insertion. 


\section{Design of an end-effector for robot-assisted ultrasound-guided breast biopsies}

Adapted from:

M. K. Welleweerd, F. J. Siepel, V. Groenhuis, J. Veltman, and S. Stramigioli, "Design of an end-effector for robot-assisted ultrasound-guided breast biopsies," International Journal of Computer Assisted Radiology and Surgery, vol. 15, no. 4, pp. 681-690, Apr. 2020. DOI: $10.1007 /$ s11548-020-02122-1

URL:

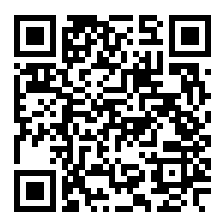




\begin{abstract}
Purpose: The biopsy procedure is a crucial phase in breast cancer diagnosis. Accurate breast imaging and precise needle placement are crucial in lesion targeting. This paper presents an end-effector (EE) for robotic 3D ultrasound (US) breast acquisitions and US-guided breast biopsies. The EE guides the needle to a specified target within the US plane. The needle is controlled in all degrees of freedom (DOFs) except for the direction of insertion, which the radiologist controls. It determines the correct needle depth and stops the needle accordingly.

Method: In the envisioned procedure, a robotic arm localizes the breast, acquires and reconstructs the 3D US volume, identifies the target and guides the needle. Therefore, the EE is equipped with a stereo camera setup, a picobeamer, US probe holder, a 3-DOFs needle guide and a needle stop. The design was realized with prototyping techniques. Experiments were performed to determine needle placement accuracy in-air. The EE was placed on a 7-DOFs robotic manipulator to determine the biopsy accuracy on a cuboid phantom.

Results: Needle placement accuracy was $0.3 \pm 1.5 \mathrm{~mm}$ in and $0.1 \pm 0.36 \mathrm{~mm}$ out of the US plane. The accuracy of the needle depth regulation was $100 \mu \mathrm{m}$ (maximum error $0.89 \mathrm{~mm}$ ). The maximum holding force of the stop was approximately $6 \mathrm{~N}$. The system reached a Euclidean distance error of $3.21 \mathrm{~mm}$ between the needle tip and the target and a normal distance of $3.03 \mathrm{~mm}$ between the needle trajectory and the target.

Conclusion: An all-in-one solution was presented which, attached to a robotic arm, assists the radiologist in breast cancer imaging and biopsy. It has a high needle placement accuracy, yet the radiologist is in control like in the conventional procedure.
\end{abstract}




\subsection{Introduction}

Breast cancer is the most prevalent cancer in women worldwide. In 2018 alone, nearly 2.1 million new cases were diagnosed [28]. It is essential for these women that the diagnosis is confirmed in an early stage of the disease as early detection reduces mortality rates in breast cancer $[124]$.

Several methods are used to detect lesions, including self-examination through palpation, and imaging modalities such as mammography, ultrasound (US) scans, and magnetic resonance imaging (MRI) scans. Mammography is the most common imaging modality in clinical practice.

A tissue sample is required to confirm malignancy if a lesion is detected. This tissue sample is acquired using a biopsy needle, after which the sample is sent to the pathologist. Primarily, the biopsy procedure is performed under US guidance. The radiologist navigates the needle based on US feedback. Disadvantages of this procedure include difficulties in extracting cells from the lesion due to its small size or poor sensitivity due to challenges in visualizing tumors against a background of dense fibroglandular tissue [125]. Also, needle insertion is hampered by tissue boundaries and lesion displacement because of forces exerted during needle insertion. The biopsy is repeated if the lesion is not hit at the previous attempt.

Consequently, radiologists should be experienced to be successful. However, clinicians who frequently use this technique often suffer from fatigue and workrelated musculoskeletal discomfort [126]. These work-related issues will become more frequent since the number of breast biopsies is increasing due to broader access to population screenings for breast cancer.

Robotics can play a essential role in these challenges; robots can more accurately, precisely and stably manipulate tools than humans. Moreover, robots do not experience fatigue, and consequently, the time per patient can be brought down 95 . Furthermore, a robotically-steered US probe can create accurate 3D US volume reconstruction. The robot can acquire the US probe position with high precision utilizing its sensors, and can produce uniformly-spaced slices with coordinated movements. The accuracy of a biopsy benefits of image fusion of preoperative images, e.g., MRI, with intraoperative data, like US [60]. If the robot "knows" its relative position to the breast and can generate a precise 3D US volume, this can ease registration. Because of these advantages, a robot-assisted US-guided biopsy can potentially reduce the number of false negatives compared to the regular procedure, and can bring down patient discomfort and costs.

Thus, robotic assistance during US-guided breast biopsies is beneficial by providing a stable hand and real-time image feedback. The previous studies focused mainly on designing mechanisms to assist the radiologist to perform minimally invasive procedures more accurately. Determining the target's position relative to the biopsy device is an important step in a robot-assisted biopsy. This position can be retrieved by registering preoperative images with the robot 
and the patient. Several studies utilized optical tracking to relate preoperative images to the robot [83, 84, 97, 98]. Nelson et al. [102] used a laser scanner to register a preoperative 3D US acquisition to the current position of the breast. The advantage of using just preoperative imaging is that the trajectory planning is not influenced or restricted by, e.g., the US probe position. However, the procedure lacks real-time information to correct for deformations. Several studies utilized real-time US guidance as well. The US probe's position relative to the needle can be tracked optically, calculated based on joint sensors of the robot(s) holding the probe or the needle, or measured if the position of the US probe is static with respect to the needle base frame $89,97,99,100,127$.

Additionally, there are several approaches to needle insertion under US guidance. Liang et al. 127] presented a six degrees of freedom (DOFs) robot holding a 3D US probe with the needle fixed to the probe. Mallapragada et al. 112, 114 showed a needle with a fixed insertion orientation relative to the probe but manipulated the tissue. Other studies suggested setups in which the needle/needle guide has some degrees of freedom in the image plane of the US probe $88,89,100,128,130$. In some cases, the needle had DOFs out of the US plane, or the US probe had DOFs also 74, 101, 131. If the needle moves independently of the US probe, there are more options for targeting lesions. However, the US feedback is less accurate if the needle moves out of the US plane.

The studies mentioned above show that the introduction of robotics to the biopsy workflow is advantageous for the accuracy of the procedure. However, to truly benefit from developments in robotics, such as the medically certified robotic arms, there is the need for an all-in-one solution. Suppose one tool enables a robotic arm to perform all steps of the breast biopsy autonomously. In that case, the system becomes less complex and expensive, and inter-system calibration errors are ruled out. These aspects will lead to higher accuracy and faster acceptance in the medical world $[132$.

This paper aims to present the design of an end-effector (EE) for utilization in a robot-assisted breast biopsy. The EE contains an actuated needle guide that directs the needle to a specified target within the US plane. The radiologist performs the needle insertion, which assures a human is still in control during the invasive step. The EE tracks the insertion and mechanically stops the needle at the specified depth. With the proposed system, MR-detected lesions may be targeted by a US-guided biopsy based on a registration step, which is less invasive than an MR-guided biopsy. Furthermore, biopsies can be consistently and reliably performed independently of the radiologist's experience in performing a biopsy. The paper is structured as follows: section 2.2 gives an analysis of the design constraints. section 2.3 presents the proposed and implemented design. section 2.4 presents the measurements performed to characterize the system, and section 2.5 discusses the results. The paper concludes with section 2.6 


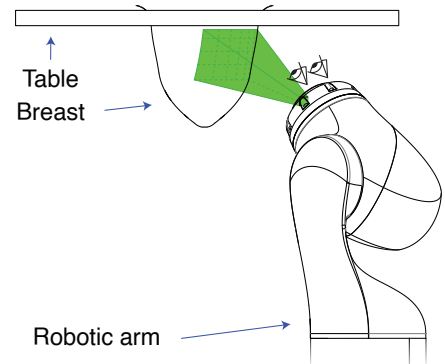

(a)

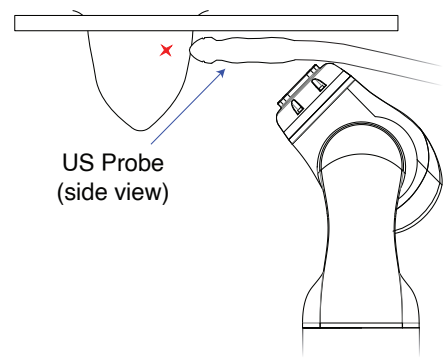

(c)

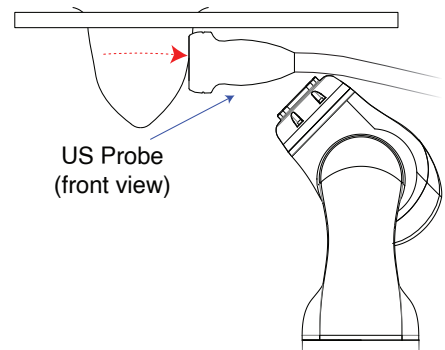

(b)

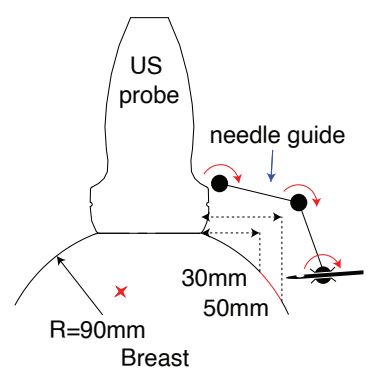

(d)

Figure 2.1: Robot-assisted biopsy workflow. (a) The robot scans the breast with cameras and registers the breast surface by projecting light or recognizing markers. (b) The robot scans the breast with a 2D US probe for 3D US volume reconstruction. (c) The robot visualizes the target in the US image. (d) The robot targets the lesion by aiming the needle guide to the correct location. In situations (b) and (c) an angle of $45^{\circ}$ of the probe with respect to the flange is beneficial to navigate close to the chest wall/patient bed.

\subsection{Design Analysis}

The envisioned robot-assisted US-guided biopsy procedure consists of several phases (Figure 2.1). First, a breast MRI is acquired in prone position. Then, the patient is positioned in prone position over the robot. This position reduces motion artifacts and simplifies registration with the preoperative MRI scan. Multi-modality markers, visible in MRI, US and on camera, should be attached to the breast to aid registration.

The robot determines its position relative to the breast by moving around it and detecting the markers with cameras attached to the end-effector (Figure 2.1(a)). Next, the MRI data are registered with the optical data. Possible deformations compared to the preoperative MRI data can be compensated for using the markers' relative positions and projections of a projector.

Subsequently, the robot scans the breast surface with a 2D linear probe to acquire 3D US data. The volume is built up by streaming the $2 \mathrm{D}$ images with corresponding position data to a reconstruction algorithm. Navigating close to 
the bed is essential to optimize the scanning area. Therefore, the probe should be tilted with respect to the robot flange (see Figure 2.1(b)).

The needle tip should be within the US transducer's field of view (FOV) during insertion. This allows for real-time image feedback of the needle tip and tissue deformations. The needle tip should be aligned with the lesion in the breast and approximately parallel with the transducer array of the US probe for needle visibility. Therefore, the needle will be inserted around $3-5 \mathrm{~cm}$ from the edge of the transducer. Furthermore, the needle is preferably inserted parallel to the chest wall because this reduces the risk for a pneumothorax. Due to these requirements, the anticipated pose of the probe during a biopsy is as shown in Figure 2.1(c).

The lesion will be a point in the 2D US image if the US probe is correctly placed on the breast surface. The target and the insertion position determine the orientation and position of the needle guide. Therefore, a 3-DOFs articulated needle guide suffices to correctly aim the needle toward the lesion in the US image plane (Figure 2.1(d)). The method to determine the joint angles based on the needle guide's position and orientation is described in 133. The guidelines for needle insertion and the diameter of the female breast, which can be up to $18 \mathrm{~cm}$ [134], define the desired workspace of the manipulator. The needle guide should successfully target lesions with a size ranging from 4 to $10 \mathrm{~mm}$. Commonly, these lesions are difficult to detect on US images but can be recognized on MRI 46. The needle is inserted through the needle guide, which limits the movement of the needle to the direction of insertion. The needle guide should stop and hold the needle at the desired depth, regardless of needle length and diameter. The brake should exert forces higher than the insertion forces to stop the needle. These forces have a range of $0-3.5 \mathrm{~N}$ [135, 136]. Preferably, the mechanism is substituted or sterilized easily after usage.

\subsection{End-effector}

\subsubsection{Design}

An overview of the proposed end-effector design is shown in Figure 2.2 The design was adapted for a KUKA MED 7 R800 (KUKA GmbH, Germany) and optimized for the phases described in the previous section. The US probe is rotated relative to the robot flange - the tool mounting surface - to move close to the patient table in the scanning and biopsy phases. The probe holder can be exchanged to support different probe types. Cameras (KYT-U200-SNF01, Kayeton Technology Co., Ltd, China) and a projector (SK UO Smart Beam, Innoio, S. Korea) are installed to support the localization phase. The stereo camera has wide-angle lenses (focal length $2.8 \mathrm{~mm}$ ) to cover a wide area regardless of the proximity to the breast surface. The cameras are synchronized for accurate stereo vision 


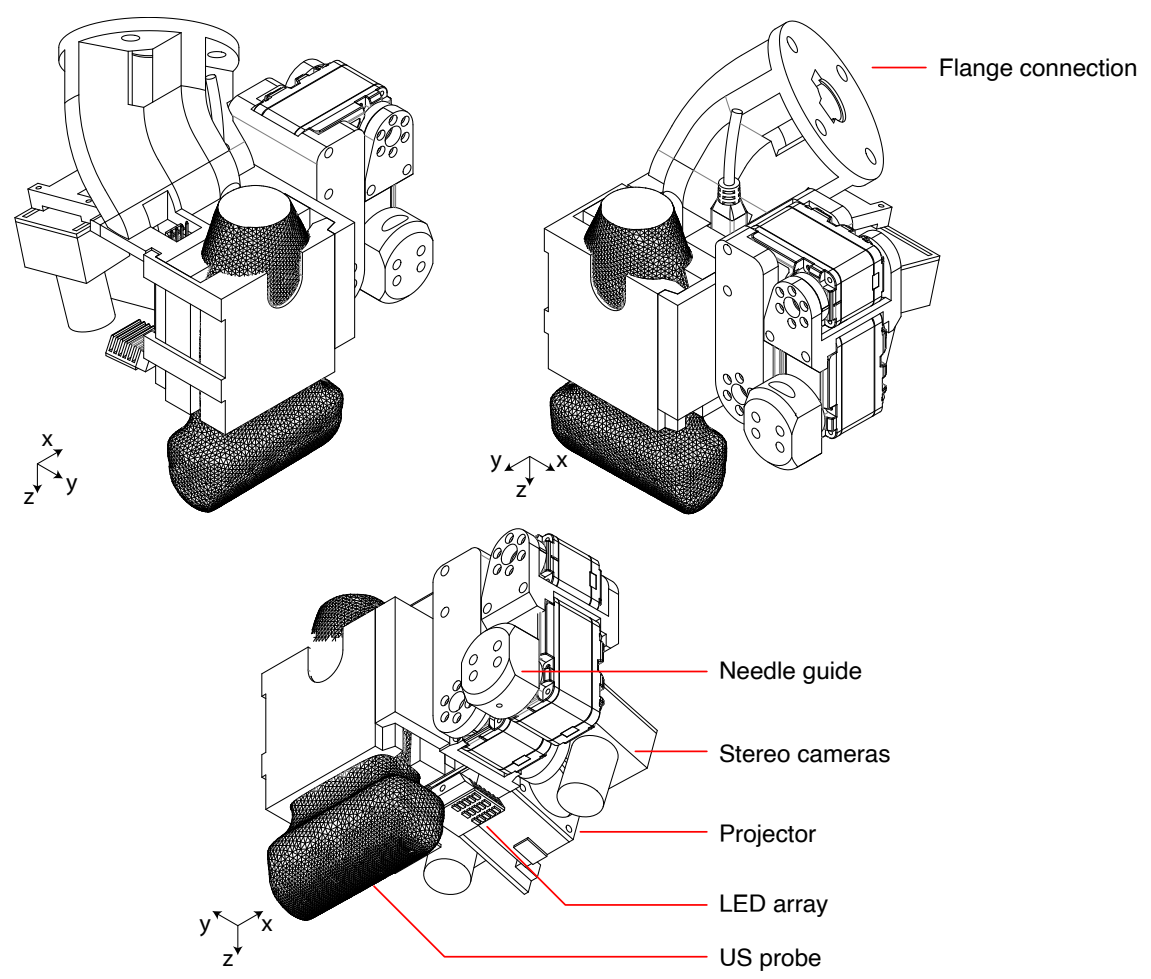

Figure 2.2: Isometric projections of the end-effector design. The US probe tip is rotated $45^{\circ}$ with respect to the robot flange around both $\mathrm{x}$ - and $\mathrm{y}$-axes. Further indicated are the needle guide, stereo cameras, projector, LED array and the US probe.

on a moving frame. Two light-emitting diode (LED) arrays are placed next to the cameras to support in segmentation of the colored markers. The cameras segment the colored markers applied to the patient's skin or phantom during camera scanning. When both cameras image the same marker, the position of the marker centroid relative to the cameras is determined. After scanning, the marker centroids relative to the robot are known and are registered with the marker centroids selected in the MRI scan (or computer-aided design (CAD) data of a phantom). This way, the lesion's location in MRI or phantom coordinates can be transformed to robot coordinates.

The needle guidance is performed by a 3-DOFs manipulator consisting of two links and a needle guide. The motors have integrated controllers, a range of $320^{\circ}$, and a resolution of $0.325^{\circ}$ (Herkulex DRS 0201, DST Robot Co., Ltd, S. Korea). Figure 2.3 highlights the 3-DOFs manipulator and its workspace. The maximum Euclidean error between the needle tip and the target in the range $\mathrm{x}$ $=[-25,25] \mathrm{mm}$ and $\mathrm{z}=[-15,45] \mathrm{mm}$ is expected to range from 0.7 to $1.1 \mathrm{~mm}$, 


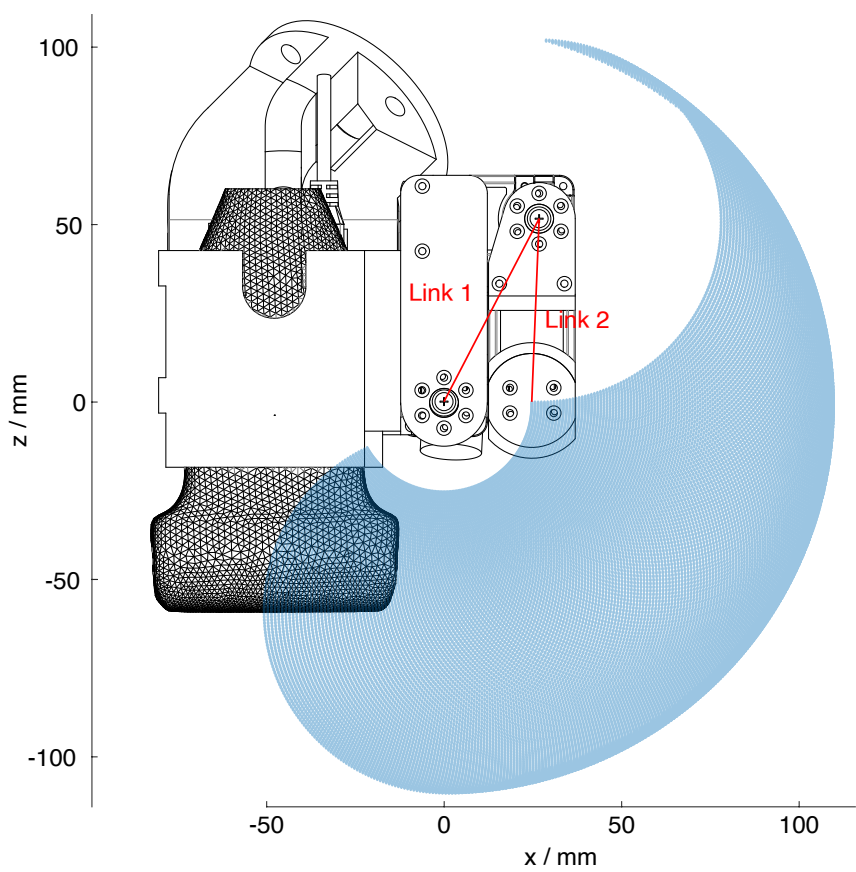

Figure 2.3: 3-DOFs motorized needle guide. Link 1 is $57.09 \mathrm{~mm}$, and link 2 is $50.36 \mathrm{~mm}$. The blue area indicates the workspace of the guide. The origin is located in the joint of the first motor.

based on the motor accuracy and the forward kinematics of the system. The error increases as the distance between the needle guide and the lesion increases. A printed circuit board (PCB) integrates a microcontroller $(\mu \mathrm{C})(\mathrm{ESP} 8266$, Espressif Systems, China), supplies for the cameras, the picobeamer and the motor, LED drivers and communication with the robot controller. The $\mu \mathrm{C}$ was programmed in the Arduino IDE (Arduino AG, Italy) to take serial commands from the robot controller and control the motors, LEDs and the needle stop. The board has separate supplies for the $\mu \mathrm{C}$ and the motors such that the robot controller can shut down the motors in case of emergency. At the same time, the communication with the end-effector continues.

An overview of the needle-stopping system is shown in Figure 2.4 The needle movement is limited to the insertion direction by matching the guide diameter with the needle diameter. The guide was partly made of hard plastic, which forms a chamber together with a flexible type of plastic. The needle is stopped by pressurizing the chamber and deforming the flexible part of the guide. This process creates friction forces that stop the needle. The following equation relates the change in the inner radius $\delta r(\mathrm{~m})$ of a tube to the pressure difference 


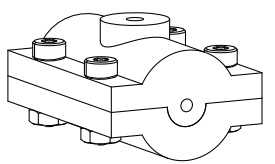

(a)

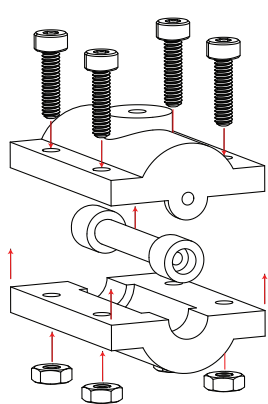

(b)

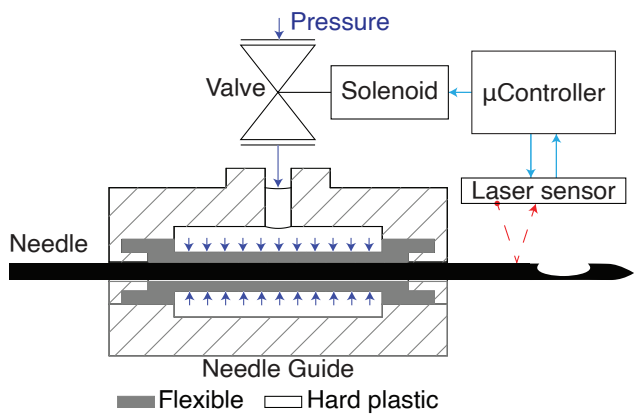

(c)

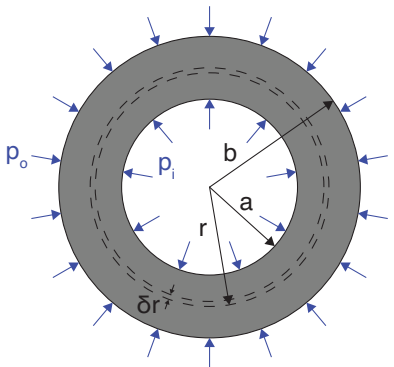

(d)

Figure 2.4: (a) The needle stop. (b) An exploded view of the needle stop. (c) A schematic diagram and a cross-section of the needle stop. A laser sensor measures the needle position, and the microcontroller controls the pressure with a solenoid-operated valve based on this position. (d) The change in tube diameter due to pressure difference across the inside and outside of the tube, see Equation 2.1.

on the inner and outer wall and its material properties 137, 138:

$$
\delta r=\frac{1-\nu}{E}\left(\frac{a^{2} p_{\mathrm{i}}-b^{2} p_{\mathrm{o}}}{b^{2}-a^{2}}\right) r+\frac{1+\nu}{E}\left(\frac{a^{2} b^{2}\left(p_{\mathrm{i}}-p_{\mathrm{o}}\right)}{b^{2}-a^{2}}\right)\left(\frac{1}{r}\right),
$$

in which $p_{\mathrm{o}}$ and $p_{\mathrm{i}}$ are the pressures on the outside and the inside of the tube $(\mathrm{Pa})$, $r$ is the initial radius of the tube $(\mathrm{m}), E$ is the Young's modulus of the material $(\mathrm{Pa}), \nu$ is the Poisson's ratio of the material, and $a$ and $b$ are the inner and the outer radius of the tube $(\mathrm{m})$. For a tube with an inner radius of $0.75 \mathrm{~mm}$ and pressures in the range of $0-6 \times 10^{5} \mathrm{~Pa}$, a wall thickness of $0.75 \mathrm{~mm}$ is sufficiently small to enable clamping the needle. A laser sensor (PAT9125, PixArt Imaging Inc., Taiwan) measures the needle displacement during insertion with a resolution of $20 \mu \mathrm{m}$. Based on the forward kinematics of the system, the $\mu \mathrm{C}$ determines the position of the needle tip during insertion. Once the needle tip has reached the target, the controller opens a pneumatic valve (PV3211-24VDC-1/8, FESTO Didactic GmbH \& Co. KG, Germany). 


\subsubsection{Realization}

Figure 2.5 presents the assembled EE. The top picture shows the EE with red arrows indicating the relevant parts. Similarly, the needle stop is shown on the bottom.

All structural parts, e.g., the links and the housing, of the end-effector are printed by fused deposition modeling printers, a Fortus 250MC (Stratasys Ltd., USA) and an Ultimaker S5 (Ultimaker, The Netherlands). The materials used are acrylonitrile butadiene styrene (ABS) (ABSplus, Stratasys, Ltd., USA) and polylactic acid (PLA) (Ultimaker, The Netherlands). The needle guide is printed utilizing an Objet Eden 260VS (Stratasys Ltd., USA). The hard plastic is VeroClear (Stratasys Ltd., USA), whereas the flexible plastic is Agilus Black (Stratasys Ltd., USA).

\subsection{Experimental Validation}

\subsubsection{Experimental Methods}

An experiment was designed to verify the needle guide's accuracy and precision in guiding the needle to a coordinate in the US image (Figure 2.6). This experiment was performed in the air to exclude the influence of tissue. The setup consisted of a mock-up US probe adapted to hold a displaceable plate with five targets indicating $\mathrm{z}=\left[\begin{array}{lllll}19 & 29 & 39 & 49 & 59\end{array}\right] \mathrm{mm}$. This plate was fixed on five marked locations, $\mathrm{x}=\left[\begin{array}{lllll}-20 & 10 & 0 & 10 & 20\end{array}\right] \mathrm{mm}$. Thus, in total there were 25 targets (red dots, Figure 2.6(b)). The needle was inserted to each target from seven insertion locations (blue dots, Figure 2.6(b)), and the position on which the needle was in contact with the plate was recorded. The measurement accuracy was $0.5 \mathrm{~mm}$ utilizing millimeter grid paper on the plate. Every combination of insertion and target position was performed five times. A needle with a conical tip (MRI IceRod $^{\mathrm{TM}}$, Galil Medical Inc., USA) was used for optimal measurement accuracy. A MATLAB script (MathWorks, Inc., USA) commanded the motor positions and saved the measured values.

The accuracy of the needle stop is defined by how well the needle is stopped at a specified depth. Therefore, the needle was inserted ten times for different set-

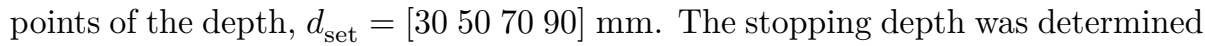
using a micro-manipulator that was moved toward the tip of the needle until the sensor on the needle guide measures contact. The measurement accuracy was approximately $10 \mu \mathrm{m}$. Furthermore, the holding force was determined using a spring balance for pressures of [2 46 6 bar.

A third experiment was designed to determine the system's accuracy (Figure 2.7). This accuracy is defined by how well the system targets a point specified in preoperative data. In a simplified setting, the CAD model of the phantom functions as preoperative data with a known shape, known marker positions, 


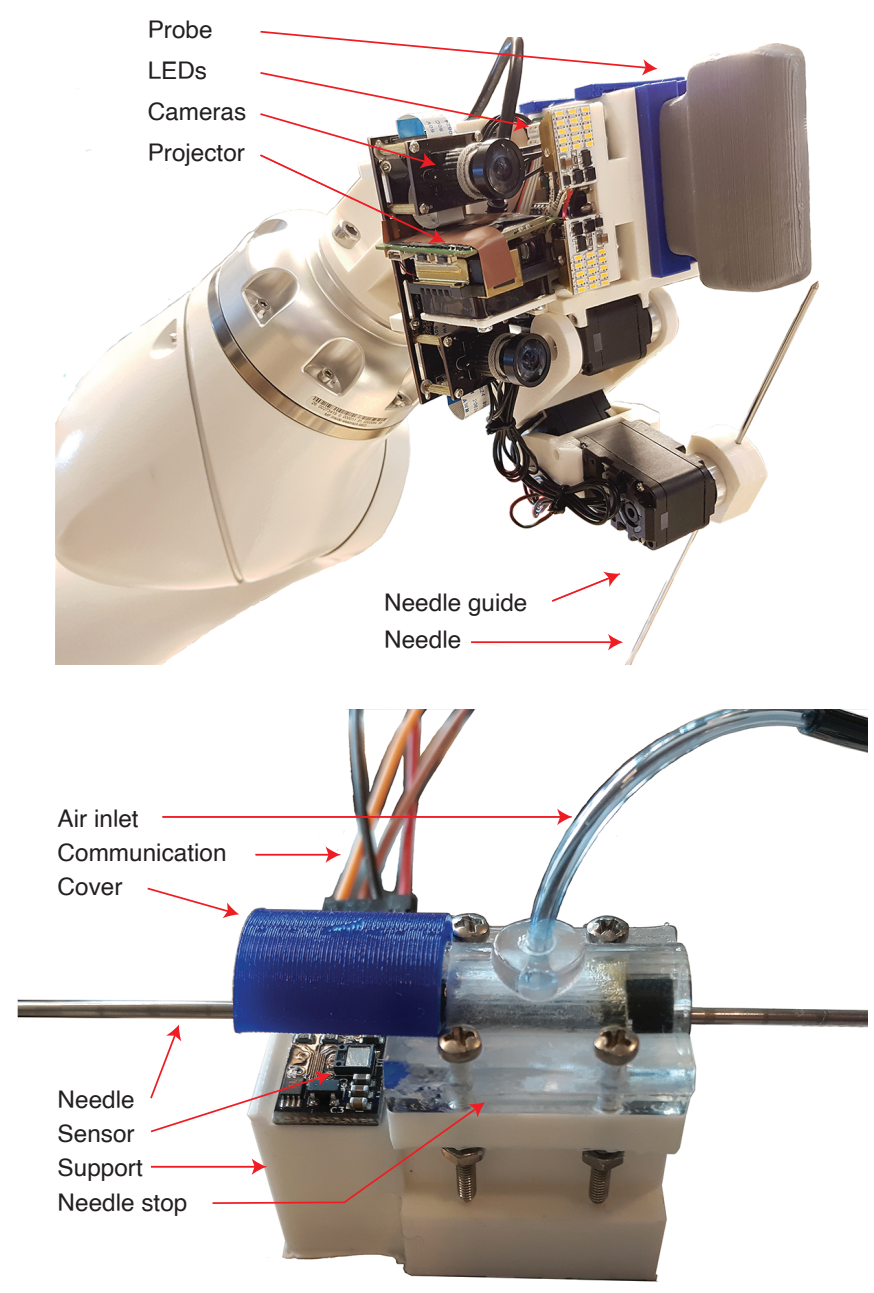

Figure 2.5: Top: the end-effector. Bottom: the needle stop. Red arrows indicate the relevant parts.

and a known lesion position. For this, a cuboid phantom $\left(6 \times 6 \times 11 \mathrm{~cm}^{3}\right)$ was constructed from candle wax (CREARTEC trend-design-GmbH, Germany). The top of a grinding sponge was integrated into the bottom to avoid back-scattering of the US signal. The phantom was placed over and registered with an Aurora tracker (Northern Digital Inc., Canada). An electromagnetic (EM) tracker (Part nr: 610065, Northern Digital Inc., Canada) is placed inside the phantom to function as the lesion, and its location with respect to the phantom is precisely known. Now, the EE was connected to a KUKA MED 7 R800. A VF13-5 linear 


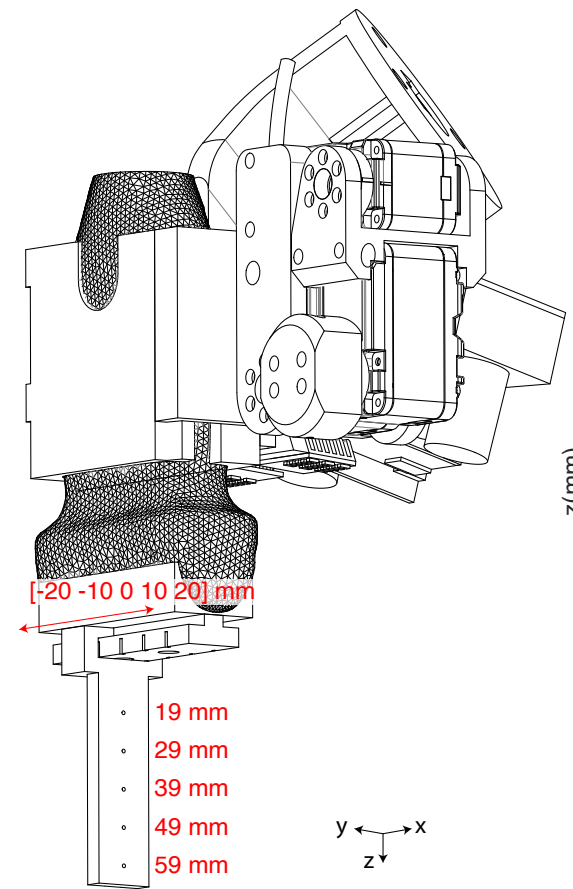

(a)

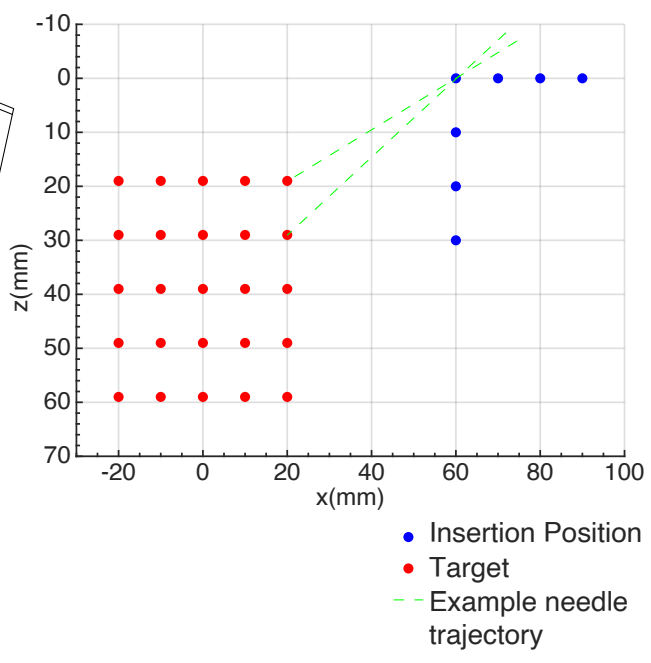

(b)

Figure 2.6: (a) Setup for measuring the accuracy and precision of the needle placement. (b) Set of targets and virtual insertion positions. The needle trajectory goes through one blue and one red point.

US probe (Siemens AG, Germany) was attached to the EE and connected to an X300 US system (Siemens AG, Germany). The robot retrieved the lesion position in robot coordinates by scanning the phantom with the cameras, determining the marker positions relative to the robot, and registering the phantom with the robot's coordinate frame. After registration, the robot moves to the phantom to perform the biopsy procedure. A custom biopsy needle was produced utilizing a metal tube with an outer diameter of $2 \mathrm{~mm}$ and an inner diameter of $1.6 \mathrm{~mm}$ and equipped with an EM tracker (Part nr: 610059). The needle is inserted to the specified position, and the Euclidean distance between the two sensors is recorded to determine the accuracy. The procedure is performed in supine position because the bed interferes with the signal of the Aurora system. The experiment was performed five times each for targets at $32.5 \mathrm{~mm}$ and $50 \mathrm{~mm}$ depth. 


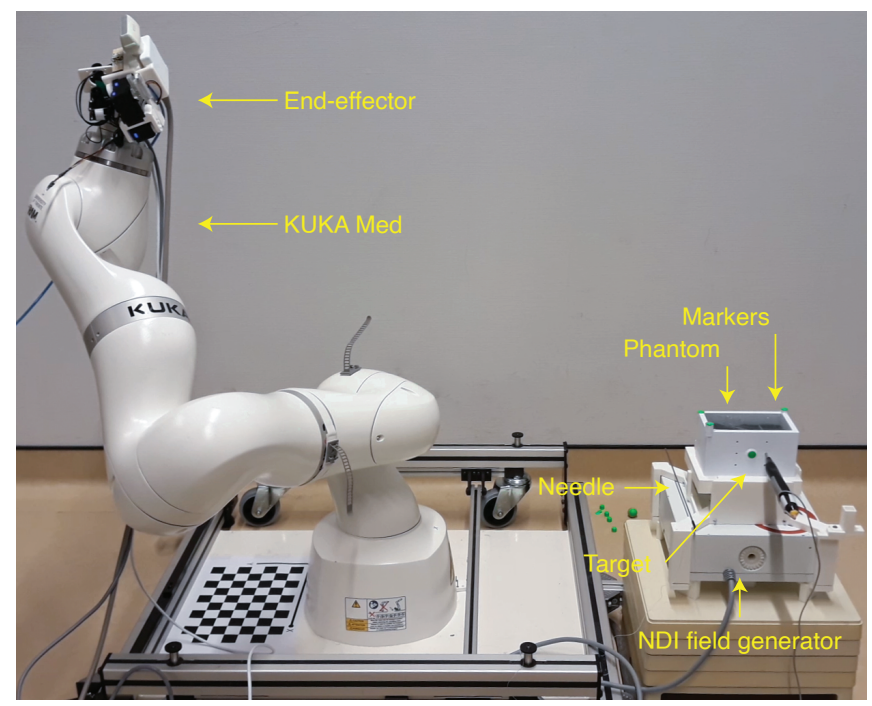

Figure 2.7: The experimental setup consists of a KUKA MED with the EE attached, a phantom with five markers placed over an NDI field generator, a target formed by an EM tracker and a needle with an integrated EM tracker.

\subsubsection{Results}

The needle guidance experiment was performed five times, of which the first dataset was used to determine the linear transformation between the measurement results and the initially targeted positions. This transformation is applied to the rest of the data, and Figure 2.8 shows the results. The red dots show the mean position for every target, while blue ellipses indicate the standard deviation in the $\mathrm{y}$ - and $\mathrm{z}$-directions. The mean error in the $\mathrm{y}$-direction and the $\mathrm{z}$-direction was $0.1 \pm 0.36 \mathrm{~mm}$ and $0.3 \pm 1.5 \mathrm{~mm}$, respectively. Target 25 was targeted the least precise, with a standard deviation of $0.48 \mathrm{~mm}$ and $1.76 \mathrm{~mm}$ in $\mathrm{y}$ - and z-directions, respectively. Furthermore, target 5 had the highest standard deviation in the z-direction, being $3.0 \mathrm{~mm}$.

Table 2.1 presents the results of the needle clamp experiment. During a calibration step, the bias of the micro-manipulator relative to the needle guide $(1.77 \mathrm{~mm})$ was removed, and the sensor's resolution was adjusted to $19.67 \mu \mathrm{m}$ through a linear fit. The accuracy in the tested range was $0.100 \mathrm{~mm}$ (maximum error $0.89 \mathrm{~mm}$ ). The holding force was determined to be $3.5-6 \mathrm{~N}$.

Table 2.2 presents the results of the phantom experiment. The Euclidean distance, $d_{\text {Euc }}$, between the needle tip and the target is $3.21 \mathrm{~mm}$ on average. The normal distance, $d_{\text {norm }}$, describes the shortest distance from the target to the needle trajectory and is $3.03 \mathrm{~mm}$ on average. The root-mean-square distance, $d_{\text {marker }}$, between the marker centroids as segmented by the cameras and modeled 


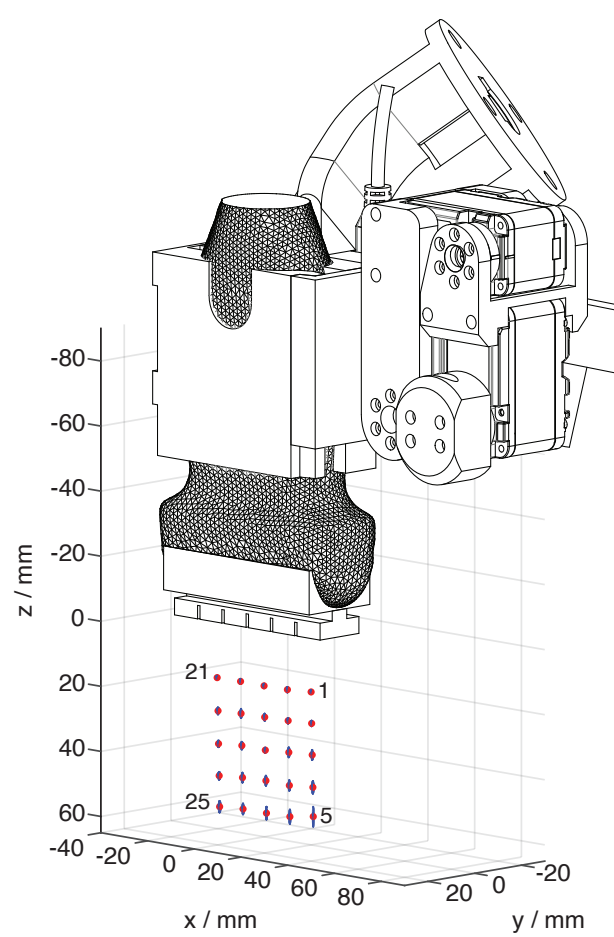

(a)

Figure 2.8: (a) The measured points plotted with the end-effector. The red dots indicate the mean, whereas the blue ellipses are formed by the standard deviation in $\mathrm{y}$ - and z-direction. (b), (c) The measured points plotted in the xz- and the yz-plane, respectively. (d) The position which was targeted the least precise, No. 25.

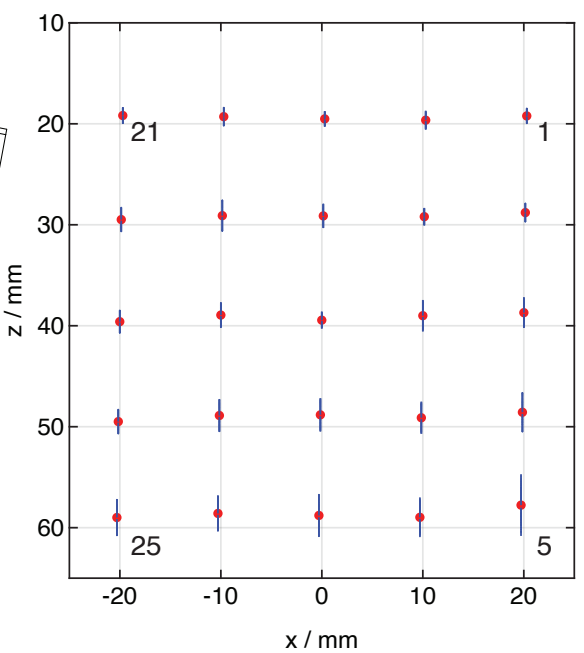

(b)

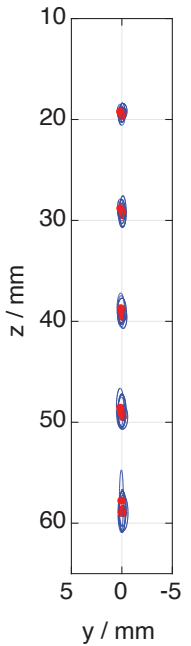

(c)

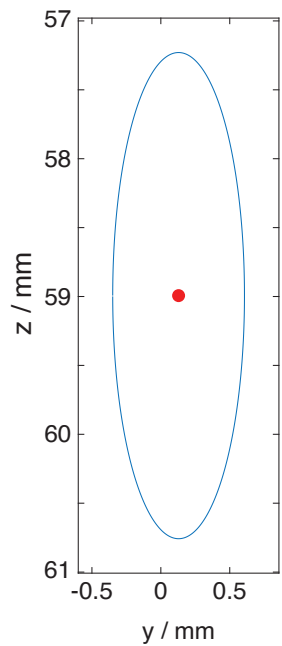

(d) 
phantom after transformation is $1.74 \mathrm{~mm}$. Figure 2.9 shows how the metal tracker and the needle insertion were visible on the US image.

\subsection{Discussion}

An EE for a robotic arm was designed to perform a robot-assisted breast biopsy workflow: registration, 3D volume acquisition and the US-guided biopsy. The presented EE integrates all necessary features in a small package. The $45^{\circ}$ angle of the US probe relative to the flange allows the robot to reach the breast near the chest wall during both the scanning and the biopsy phase. In a simplified setting, the pre- and intra-operational data could be registered utilizing the cameras and the LED arrays on the EE. Although not shown here, the picobeamer

Table 2.1: Top: The set and measured needle depths. Bottom: The applied pressure and the corresponding holding force.

\begin{tabular}{cccc}
\hline $\begin{array}{cccc}\text { Setpoint } \\
\mathrm{mm}\end{array}$ & $\frac{\text { Mean }}{\mathrm{mm}}$ & $\frac{\text { Min. }}{\mathrm{mm}}$ & $\frac{\text { Max. }}{\mathrm{mm}}$ \\
\hline 30.00 & 30.18 & 29.75 & 30.89 \\
50.00 & 50.00 & 49.82 & 50.26 \\
70.00 & 70.02 & 69.89 & 70.18 \\
90.00 & 90.20 & 90.05 & 90.35 \\
\hline
\end{tabular}

\begin{tabular}{|c|c|}
\hline Pressure & Hold force \\
\hline bar & $\mathrm{N}$ \\
\hline 2.0 & 3.5 \\
\hline 4.0 & 5.0 \\
\hline 6.0 & 6.0 \\
\hline
\end{tabular}

Table 2.2: The distance, $d$, the Euclidean distance, $d_{\text {Euc }}$, and the normal distance, $d_{\mathrm{Norm}}$, between the needle tip and the target, and the Euclidean distance between the markers after registration in the phantom experiment.

\begin{tabular}{|c|c|c|c|c|c|c|}
\hline & & & Need & & & Marker \\
\hline & & $d(x y z)$ & & $d_{\text {Euc }}$ & $d_{\mathrm{Norm}}$ & $d_{\text {Euc }}$ \\
\hline & & $\mathrm{mm}$ & & $\mathrm{mm}$ & $\mathrm{mm}$ & $\mathrm{mm}$ \\
\hline Mean & 1.03 & -2.62 & -0.11 & 3.21 & 3.03 & 1.74 \\
\hline Min. & 0.70 & -2.28 & 0.01 & 2.38 & 2.04 & 1.59 \\
\hline Max. & 2.49 & -3.70 & -1.57 & 4.72 & 4.61 & 1.85 \\
\hline
\end{tabular}




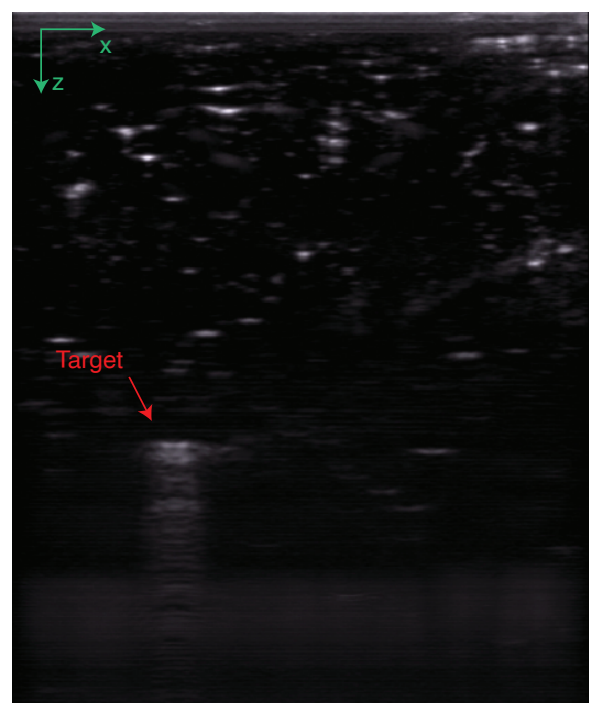

(a)

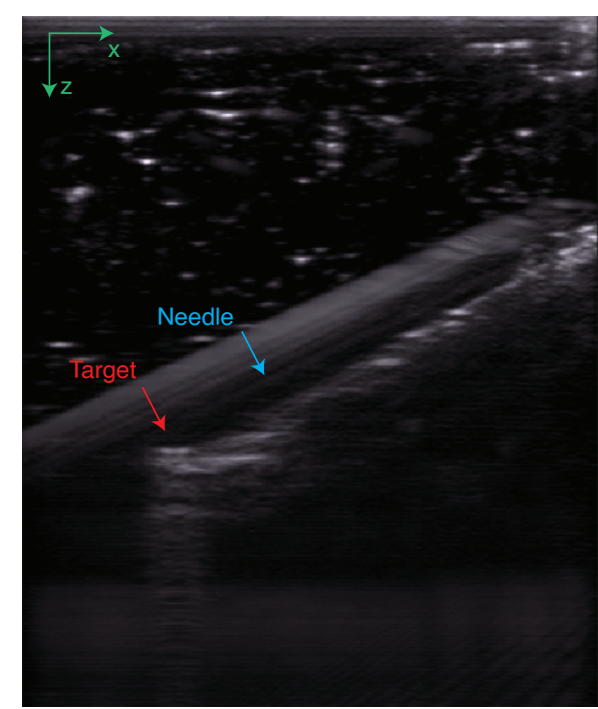

(b)

Figure 2.9: (a) The US plane containing the target. (b) The US plane containing the target after needle insertion.

could help add a deformable registration to the procedure. The 3-DOFs needle guide successfully assists in targeting a lesion location defined preoperatively. Both in-air and phantom experiments were performed to determine the needle placement accuracy. The in-air experiments showed that the needle is accurately guided to a predefined position in the US plane, and the needle is accurately stopped at a predefined depth. The phantom experiment showed that the needle trajectory has a mean normal distance of $3.03 \mathrm{~mm}$ to the target. Table 2.2 shows that a significant contribution to this error is in the y-direction, out of the US plane, while the in-plane errors are similar to the in-air experiments, which were focused on needle guidance and stopping accuracy. Furthermore, Table 2.2 shows that the camera segmentation's error is in the millimeter range. As some force was needed to insert the target in the phantom, it is suspected that this caused a small error in the phantom to field generator registration. Other factors influencing the error metric could include the accuracy of the calibrations of the needle guide, the US probe and the cameras with respect to the robot flange and the inter-camera position. Overall, the EE has similar accuracy as the cited studies $(0.25-3.44 \mathrm{~mm}$ [83, 101]), and for the system, it is feasible to target lesions in the range of $4-10 \mathrm{~mm}$ in the future.

Considering Figure 2.7, the standard deviations are larger than the mean errors since the motors have backlash in the gears. Additionally, the printed parts do not provide the same rigidity as, e.g., metal parts. Furthermore, target 
5 has a relatively large standard deviation in the z-direction because the needle reaches this target under a sharp angle. Minor deviations in target placement and the insertion angle cause rather large variations in Euclidean distance errors. Target 25 is targeted with the lowest precision since this target is located the farthest away from the needle guide. Both positions will not be used in real-life scenarios; for optimal needle and target visibility, the target is usually located more toward the center of the US image.

The system has several advantages: the biopsy site can be marked on preoperative images, and the correct biopsy site is found due to the marker recognition. The radiologist controls the insertion, yet has robotic biopsy accuracy due to the needle guide. The physician has valuable feedback when puncturing the skin and other tissue boundaries due to the frictionless movement of the needle. The displacement sensor's accuracy is satisfactory, considering that in the range of $30-90 \mathrm{~mm}$, the stopping system has an accuracy of $0.100 \mathrm{~mm}$. The laser is located away from the needle, so the needle guide is easily replaced after a biopsy or when changing the needle diameter. Furthermore, the system works independently of the needle length. Also, the needle is released when power is lost, and in case of emergency the practitioner can remove the needle by overcoming the clamping forces. This makes the system safe to use in a clinical environment.

In the current setup, possible deformations were not considered, but this was unnecessary since the target position was static. This should be implemented in future experiments where the needle insertion can displace the lesion. This may be done utilizing simulations or by tracking the needle and deformations in the US image. Needle tracking may also decrease the influence of backlash and the system's rigidity by providing feedback. Further improvements include changing the material of the clamping mechanism of the needle stop, which is too brittle. Due to the brittleness, making the instrument airtight and durable is difficult. However, this did not influence the working principle of the needle stop.

For clinical application, the procedure must be sterile. During camera scanning, the EE is not in contact with the patient. During needle insertion, the needle guide is in contact with the needle, and thus this part will be disposable. A US transparent sheet can cover the setup during the procedure to create a sterile environment.

\subsection{Conclusion and Recommendations}

This paper introduced an EE for a robotic manipulator to assist the radiologist in acquiring US breast scans and performing the US-guided biopsy. The 3DOFs needle guide with needle stop gives radiologist robotic accuracy, yet the radiologist is in control since needle insertion is not robotized.

The accuracy and precision of the 3 -DOFs needle guide were determined 
experimentally, both in the air and on a phantom. The results look promising and indicate that targeting lesions with a size of $4-10 \mathrm{~mm}$ is feasible.

The results of this study are an example of how to integrate different aspects of robotic US scanning and robot-assisted biopsy in one functional device. 


\section{Combining Geometric Workspace Compliance with Energy-based Joint Limit Avoidance}

Adapted from:

M. K. Welleweerd, S. S. Groothuis, S. Stramigioli, and F. J. Siepel, "Combining Geometric Workspace Compliance with Energy-based Joint Limit Avoidance," In preparation, 


\begin{abstract}
Robotic manipulators are utilized in and exposed to ever more complex environments. Particularly in healthcare, it is crucial to guarantee safety while navigating around the operating theater and being in contact with the patient. These robots are expected to behave in a compliant manner when interacting with their environment. Many of these robotic manipulators are redundant, which means they can perform additional tasks. Such a task could be manipulability optimization, which is often implemented by cost function descending using a pseudoinverse of the manipulator Jacobian.

Alternatively, this paper achieves manipulability optimization by introducing of a potential field generated by nonlinear virtual springs in the joints. The stored energy is released in the null space by ensuring a negative null space power. The kinetic energy is limited by dynamically scaling the spring stiffness. Joint limits are avoided by activating additional springs if joints threaten to reach a limit despite the optimization. These springs create a compliant null space behavior for which inverting the Jacobian is not necessary. Thus, the performance is not affected by singularities.

In a series of experiments and simulations, we show that the robot navigates to a local minimum of the virtual joint energy function, rendering a more neutral robot configuration. It does so while not affecting the end-effector behavior. Also, the maximum kinetic energy is respected and the joint limits are avoided with a safe margin.
\end{abstract}




\subsection{Introduction}

Situations in which robots are closely working together with humans have increased and will be increasing in the coming years. Especially in medical settings, robots should function consistently and in a compliant manner while varying obstacles and complex trajectories demand much of the robot's control. In the MRI and Ultrasound Robotic Assisted Biopsy (MURAB) project, we have been working on robotic volumetric breast ultrasound acquisitions (Figure 3.1 [139]. The MURAB project is just one example where the dexterity and precision of a redundant robotic manipulator are ideally suited for patient-specific trajectories and accurate localization of the acquired ultrasound slices. Other examples arise from the COVID-19 pandemic, where robots are deployed for disinfection, patient assistance and rehabilitation [140. In such applications, the robots are in direct contact with the patient, and thus, the trajectories can be very complex and compliant behavior is paramount 141 .

In the three-dimensional workspace, at least six independent joints are required to control the end-effector (EE) in all six degrees of freedom (DOFs) (location and orientation). Robotic manipulators often have a kinematically redundant design to give them the necessary dexterity in complex tasks and environments. Kinematic redundancy means that more DOFs (i.e., joints) are available than strictly needed for the kinematic properties of the task 1,142 . These extra DOFs can be utilized to perform additional tasks that support the robot's primary task. One possible additional task is to keep the linkage away from the joint limits. Joint limit avoidance is beneficial for ultrasound acquisitions since the joints should jointly perform a $360^{\circ}$ motion around the breast. Each individual link cannot stretch that range.

There are two ways to keep the linkage away from their limits: either to prevent the current solution from going past a limit or to optimize the robot configuration relative to its limits continuously. The former is achieved by implementing dominant joint limit avoidance when a joint is close to a limit. Its advantage is that additional DOFs can be used for other tasks while the joint positions are sufficiently far away from their limits. A drawback is that the behavior is only active near the limits, such that when a limit is reached, the solution space for further movement may be restricted. The alternative is to avoid the joint limits by optimizing a cost function continuously 1,142 . The robot will use its self-motion to traverse down the cost function's gradient and optimize the range of motion for each joint. However, this will not prevent the manipulator from reaching a limit eventually. Hence, combining the two methods may be preferred. Both will be discussed in more detail.

Joint limit avoiding behavior that is only active near the limits is implemented in several ways. Most methods utilize some function that is asymptotic on either end of the joint range. One common way is the introduction of artificial potential fields (e.g., a FIRAS function) near the joint limits, as introduced by Khatib 


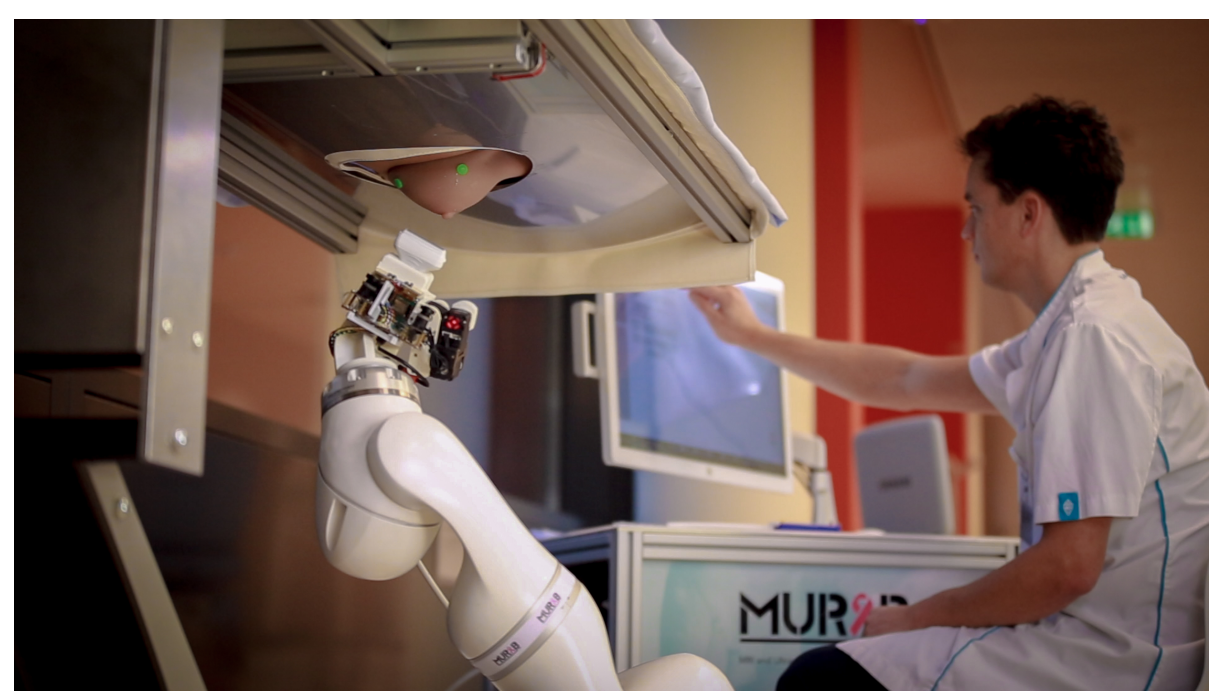

Figure 3.1: The MURAB robotic setup for patient-specific ultrasound acquisitions.

143. The force/torque generated by the field gradually increases as the joint position nears the limit and will prevent the robot from reaching them. Many researchers adopted this method, but sometimes they utilized other functions for the potential field $[144,145]$. This method elegantly pushes the manipulator away from its limits but is prone to oscillations when tracking a trajectory 146. Alternatives are the saturation in null space and the saturation in joint space methods 146, 147]. These methods predict whether a limit will be reached in the next control iteration. If so, a new task is generated that keeps the joint from reaching its limit. Another option is the implementation of barrier functions, to which the solution of the main task should comply 148,149 .

A joint space impedance controller may achieve compliant joint position optimization, but a conventional controller will not respect the desired EE behavior. Ott describes how to accomplish a null space impedance, which does not affect the task space 150. One way to solve this is the projection of the controller's torque in the null space by kinematically decoupling the joint space impedance controller from the Cartesian impedance controller 151, 152. The task space augmentation and the joint space decomposition method are alternatives. The former approach augments task coordinates, whereas the latter augments task velocities. The latter extends the Jacobian to contain additional null space coordinates and does not introduce additional singularities, which is the case for task space augmentation. The disadvantage is that null space coordinates are not geometrically meaningful. No integration of the null space velocities is required if a particular form of the extended Jacobian is chosen 153, 154]. The disadvantages of this method are the complexity and the usage of 
the Jacobian inverse, which may have discontinuities at singular configurations.

This work looks at the combination of geometric workspace compliance with energy-based joint limit avoidance. Null space compliance is achieved explicitly considering the forces associated with the gradient of the energy field introduced in the joint space. The joint positions are optimized by navigating along the gradient while ensuring a negative null space power. The kinetic energy in the null space is bounded by dynamically shaping the potential field. If a joint limit is approached, hard joint limits are realized by introducing an additional potential field. We achieve a natural and physically consistent control for which inverting the Jacobian is unnecessary by explicitly modeling all energies. The manipulator transitions smoothly in and out of singularities by remaining at an energetic minimum. We performed several simulations and experiments to verify the controller's intended behavior.

\subsection{Joint limit avoidance with joint space poten- tial energy}

Our controller consists of three main components: a regular Cartesian impedance controller expanded with joint position optimization and joint limit avoidance. Joint position optimization is defined as a tendency of the robot to achieve EE configurations while maintaining the joint positions as neutral as possible. Additionally, joint limit avoidance prevents the robot from reaching its mechanical limits. We will start with a description of the Cartesian impedance controller, after which the joint position optimization and the joint limit avoidance torques are elaborated.

\subsubsection{Impedance control}

The dynamic equation of the controlled system is given by

$$
\begin{array}{r}
\boldsymbol{M}(\boldsymbol{q}) \ddot{\boldsymbol{q}}+\boldsymbol{C}(\boldsymbol{q}, \dot{\boldsymbol{q}}) \dot{\boldsymbol{q}}+\boldsymbol{F}(\boldsymbol{q}, \dot{\boldsymbol{q}})^{\top}+\boldsymbol{G}(\boldsymbol{q})^{\top}= \\
\underbrace{\boldsymbol{J}^{\top}(\boldsymbol{q}) \boldsymbol{W}^{0^{\top}}}_{\text {elastic wrench }}+\boldsymbol{\tau}_{\mathrm{opt}}^{\top}+\boldsymbol{\tau}_{\mathrm{jla}}^{\top}+\boldsymbol{\tau}_{\mathrm{ext}}^{\top},
\end{array}
$$

in which $\boldsymbol{q} \in \mathbb{R}^{n}$ is the vector with joint positions, $n$ being the number of DOFs, $\boldsymbol{J}(\boldsymbol{q}) \in \mathbb{R}^{6 \times n}$ is the manipulator Jacobian, $\boldsymbol{M}(\boldsymbol{q}) \in \mathbb{R}^{n \times n}$ is the inertia matrix, $\boldsymbol{C}(\boldsymbol{q}, \dot{\boldsymbol{q}}) \in \mathbb{R}^{n}$ represents the Coriolis and centrifugal terms, $\boldsymbol{F}(\boldsymbol{q}, \dot{\boldsymbol{q}}) \in \mathbb{R}^{n}$ contains the friction forces, $\boldsymbol{G}(\boldsymbol{q}) \in \mathbb{R}^{n}$ is the gravitational term, and $\boldsymbol{\tau}_{\text {opt }}, \boldsymbol{\tau}_{\text {jla }}, \boldsymbol{\tau}_{\text {ext }} \in \mathbb{R}^{n}$ represent the joint position optimization, the joint limit avoidance, and the external torques, respectively. The external torques originate from external forces on the EE and intermediate bodies. In this derivation, it is assumed that the external torques are zero. The elastic wrench, $W^{0} \in{ }^{*} \operatorname{se}(3)$, is the 
virtual force exerted by a virtual spatial spring, $\boldsymbol{K} \in \mathbb{R}^{6 \times 6}$, connected between the current EE frame, $\Psi_{\text {ee }}$, and its desired frame, $\Psi_{\mathrm{d}} \cdot \boldsymbol{K}$ is comprised of a translational, a rotational and a coupling component, $\boldsymbol{K}_{\mathrm{t}}, \boldsymbol{K}_{\mathrm{o}}, \boldsymbol{K}_{\mathrm{c}} \in \mathbb{R}^{3 \times 3}$, such that the elastic wrench expressed in $\Psi_{\text {ee }}$ can be denoted as 155

$$
\boldsymbol{W}^{\mathrm{ee}}{ }^{\mathrm{T}}=\left(\begin{array}{c}
\left(\boldsymbol{m}^{\mathrm{ee}}\right)^{\mathrm{T}} \\
\left(\boldsymbol{f}^{\mathrm{ee}}\right)^{\mathrm{T}}
\end{array}\right)=\left(\begin{array}{ll}
\boldsymbol{K}_{\mathrm{o}} & \boldsymbol{K}_{\mathrm{c}} \\
\boldsymbol{K}_{\mathrm{c}}^{\top} & \boldsymbol{K}_{\mathrm{t}}
\end{array}\right)\left(\begin{array}{l}
\delta \boldsymbol{\theta}_{\mathrm{ee}}^{\mathrm{d}} \\
\delta \boldsymbol{p}_{\mathrm{ee}}^{\mathrm{d}}
\end{array}\right),
$$

where $\boldsymbol{m}^{\text {ee }}$ and $\boldsymbol{f}^{\text {ee }}$ denote the rotational and the translational part of the wrench and $\delta \boldsymbol{T}=\left[\delta \boldsymbol{\theta}_{\mathrm{ee}}^{\mathrm{d}}{ }^{\mathrm{T}} \delta \boldsymbol{p}_{\mathrm{ee}}^{\mathrm{d}}\right]^{\mathrm{T}} \in \mathrm{se}(3)$ is an infinitesimal twist in vector form. The positions of $\Psi_{\mathrm{ee}}$ and $\Psi_{\mathrm{d}}$ with respect to the base frame, $\Psi_{0}$, are represented by the homogeneous matrices $\boldsymbol{H}_{\mathrm{ee}}^{0}, \boldsymbol{H}_{\mathrm{d}}^{0} \in \mathrm{SE}(3)$, respectively. As such, the pose of the EE with respect to the desired frame can be obtained by

$$
\boldsymbol{H}_{\mathrm{ee}}^{\mathrm{d}}=\left(\boldsymbol{H}_{\mathrm{d}}^{0}\right)^{-1} \boldsymbol{H}_{\mathrm{ee}}^{0}=\left(\begin{array}{cc}
\boldsymbol{R}_{\mathrm{ee}}^{\mathrm{d}} & \boldsymbol{p}_{\mathrm{ee}}^{\mathrm{d}} \\
0 & 1
\end{array}\right),
$$

where $\boldsymbol{R}_{\mathrm{ee}}^{\mathrm{d}} \in \mathrm{SO}(3)$ represents the rotation matrix and $\boldsymbol{p}_{\mathrm{ee}}^{\mathrm{d}} \in \mathbb{R}^{3}$ the translation vector. Additionally, based on the components of the stiffness matrix, three co-stiffness matrices, $\boldsymbol{G}_{\mathrm{t}}, \boldsymbol{G}_{\mathrm{o}}, \boldsymbol{G}_{\mathrm{c}} \in \mathbb{R}^{3 \times 3}$, can be defined such that:

$$
\boldsymbol{G}_{x}=\frac{1}{2} \operatorname{tr}\left(\boldsymbol{K}_{x}\right) \boldsymbol{I}^{3 \times 3}-\boldsymbol{K}_{x} \text { for } x=\mathrm{t}, \mathrm{o}, \mathrm{c} .
$$

In Equation (3.4), the $\operatorname{tr}()$ operator is the trace operator, which takes the sum of the elements on the diagonal of a square matrix. Next, the skew symmetric form, indicated by the tilde-operator, of the torque $\boldsymbol{m}^{\mathrm{ee}}$ and force $\boldsymbol{f}^{\mathrm{ee}}$ can be calculated as:

$$
\begin{aligned}
\tilde{\boldsymbol{m}}^{\mathrm{ee}}= & -2 \operatorname{as}\left(\boldsymbol{G}_{\mathrm{o}} \boldsymbol{R}_{\mathrm{ee}}^{\mathrm{d}}\right)-\operatorname{as}\left(\boldsymbol{G}_{\mathrm{t}} \boldsymbol{R}_{\mathrm{d}}^{\mathrm{ee}} \tilde{\boldsymbol{p}}_{\mathrm{ee}}^{\mathrm{d}} \tilde{\boldsymbol{p}}_{\mathrm{ee}}^{\mathrm{d}} \boldsymbol{R}_{\mathrm{ee}}^{\mathrm{d}}\right) \\
& -2 \operatorname{as}\left(\boldsymbol{G}_{\mathrm{c}} \tilde{\boldsymbol{p}}_{\mathrm{ee}}^{\mathrm{d}} \boldsymbol{R}_{\mathrm{ee}}^{\mathrm{d}}\right) \\
\tilde{\boldsymbol{f}}^{\mathrm{ee}}= & -\boldsymbol{R}_{\mathrm{d}}^{\mathrm{ee}} \operatorname{as}\left(\boldsymbol{G}_{\mathrm{t}} \tilde{\boldsymbol{p}}_{\mathrm{ee}}^{\mathrm{d}}\right) \boldsymbol{R}_{\mathrm{ee}}^{\mathrm{d}}-\operatorname{as}\left(\boldsymbol{G}_{\mathrm{t}} \boldsymbol{R}_{\mathrm{d}}^{\mathrm{ee}} \tilde{\boldsymbol{p}}_{\mathrm{ee}}^{\mathrm{d}} \boldsymbol{R}_{\mathrm{ee}}^{\mathrm{d}}\right) \\
& -2 \operatorname{as}\left(\boldsymbol{G}_{\mathrm{c}} R_{\mathrm{ee}}^{\mathrm{d}}\right)
\end{aligned} .
$$

Here, as () is an operator that gives the anti-symmetric part of a square matrix. Finally, the wrench, $\boldsymbol{W}^{\mathrm{ee}}$, exerted on the EE by the spring is expressed in the base frame by

$$
\boldsymbol{W}^{0^{\top}}=\operatorname{Ad}_{\boldsymbol{H}_{0}^{\mathrm{ee}}}^{\top} \boldsymbol{W}^{\mathrm{ee}}{ }^{\top},
$$

where Ad is the Adjoint of an element of $\mathrm{SE}(3) . \boldsymbol{H}_{0}^{\text {ee }}$ is the base frame expressed in the EE frame.

The control law for the actuator torques, $\tau_{\mathrm{a}}$, then becomes

$$
\tau_{\mathrm{a}}^{\top}=\boldsymbol{J}^{\top}(\boldsymbol{q}) \boldsymbol{W}^{0^{\top}}-\hat{\boldsymbol{G}}(\boldsymbol{q})^{\top}-\hat{\boldsymbol{C}}(\boldsymbol{q}, \dot{\boldsymbol{q}}) \dot{\boldsymbol{q}}+\boldsymbol{\tau}_{\mathrm{opt}}^{\top}+\boldsymbol{\tau}_{\mathrm{jla}}^{\top},
$$


in which $\hat{\boldsymbol{G}}(q)$ is a compensation for the gravitational forces and $\hat{\boldsymbol{C}}(\boldsymbol{q}, \boldsymbol{\boldsymbol { q }}) \dot{\boldsymbol{q}}$ is a compensation for the Coriolis and centrifugal forces. The friction forces, $\boldsymbol{F}(\boldsymbol{q}, \dot{\boldsymbol{q}})$, as present in Equation (3.1), cannot be compensated since they are unknown. $\tau_{\text {opt }}$ and $\tau_{\text {jla }}$ will be defined in the following sections.

\subsubsection{Joint position optimization}

We can define a potential energy function based on the robot's joint positions that increases as joints move away from their neutral position. Therefore, a stiffness matrix is defined in joint $\operatorname{space}^{1} \boldsymbol{K}_{\mathrm{q}}(\boldsymbol{q}) \in \mathbb{R}^{n \times n} \cdot \boldsymbol{K}_{\mathrm{q}}(\boldsymbol{q})$ is a diagonal matrix; thus, the total energy of all the springs in the joints is defined as

$$
E_{\mathrm{t}}(\boldsymbol{q})=\sum_{i=1}^{n} \int_{0}^{q_{\mathrm{i}}} k_{i i}\left(q_{i}\right) q_{i} d q_{i}
$$

where $k_{i i}\left(q_{i}\right)$ is the $(i, i)$-th element of $\boldsymbol{K}_{\mathrm{q}}(\boldsymbol{q})$ which is described by

$$
k_{i i}\left(q_{i}\right)=\frac{\alpha_{i}}{1-\cos \left(\frac{q_{i} \pi}{q_{\lim _{i}}}+\pi\right)},
$$

where $q_{i}, q_{\lim _{i}}$ and $\alpha_{i}$ are the joint position, the joint limit and a scaling term for the stiffness of the $i$-th joint, respectively. Here, we assume that each joint has symmetric limits, such that the minimal joint position is given by $-q_{\lim _{i}}$. Figure 3.2 shows the graph of Equation 3.9 for $\alpha=1$ and $q_{\text {lim }}=170^{\circ}$.

The manipulator releases spring energy by moving to a more neutral position. Figure 3.2 indicates that the potential energy increase/decrease associated with moving a joint that is currently closer to a joint limit is more significant. However, the robot should release the potential energy only in its null space since the EE task must not be compromised. The possible joint motions of a redundant manipulator that do not change the EE configuration are given by linear combinations of the null space vectors of the Jacobian $\boldsymbol{J}(\boldsymbol{q})$, i.e.:

$$
\operatorname{null}(\boldsymbol{J}(\boldsymbol{q}))=\left\{\dot{\boldsymbol{q}} \in \mathbb{R}^{n \times 1} \mid \boldsymbol{J}(\boldsymbol{q}) \dot{\boldsymbol{q}}=0\right\} .
$$

The KUKA LBR Med has seven joints $(n=7)$ such that the null space of this robot is given by a $7 \times 1$ vector indicating the direction of combined and simultaneous joint motions that will not affect the EE position, which we will denote by $\dot{\boldsymbol{q}}^{0}$. The gradient of Equation 3.8 in the direction of the null-space vector gives the magnitude of the force experienced when moving along the null space vector:

$$
\nabla_{\dot{\boldsymbol{q}}^{0}} E_{\mathrm{t}}(\boldsymbol{q})=\left(\boldsymbol{K}_{\mathrm{q}}\left(\boldsymbol{q}_{\mathrm{home}}-\boldsymbol{q}\right)\right)^{\top} \cdot \dot{\boldsymbol{q}}^{0}
$$

\footnotetext{
${ }^{1}$ Although deriving the stiffness from the energy field may have been more natural, desired stiffness behavior is more intuitive to design.
} 


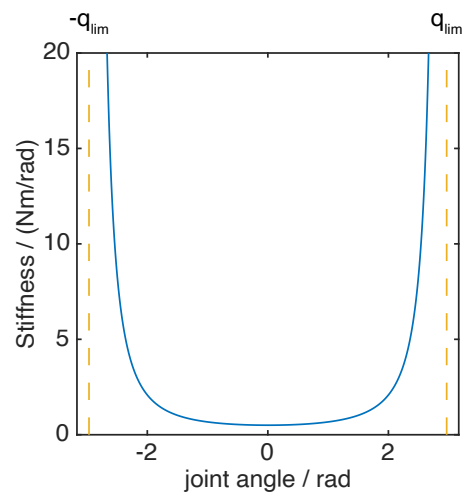

Figure 3.2: Example plot of the spring stiffness as a function of the joint angle for a given joint and its joint limits.

Here, $\boldsymbol{q}_{\text {home }}$ is the home position of the robot, in which all joints are at their neutral position. The null space power is the product of the null space torque, $\boldsymbol{\tau}_{0}$, and the null space joint velocity. The null space power should be negative to release energy; thus, the torque vector should oppose the null space vector:

$$
\boldsymbol{\tau}_{0}^{\top}=-\nabla_{\dot{\boldsymbol{q}}^{0}} E_{\mathrm{t}}(\boldsymbol{q}) \boldsymbol{M}(\boldsymbol{q}) \dot{\boldsymbol{q}}^{0}
$$

Next, we introduce a joint space damping, $\boldsymbol{D}_{\mathrm{q}} \in \mathbb{R}^{7 \times 7}$, which is not constant, but a function of $\boldsymbol{M}(q)$ (see Table 3.1), to ensure the accelerations take place in the null space [156]. By doing so, the joint space velocity would saturate to a certain $\dot{\boldsymbol{q}}_{\text {sat }}$, because the torque generated by the spring and the counter-torque generated by the damper are in balance:

$$
\dot{\boldsymbol{q}}_{\mathrm{sat}}=\boldsymbol{D}_{\mathrm{q}}^{-1} \boldsymbol{\tau}^{0}
$$

Thus, the joint velocities generated by the virtual springs can be limited such that $\left\|\dot{\boldsymbol{q}}_{\text {sat }}\right\|=\dot{q}_{\max }$. This is done by scaling the torque with a factor $v$, in a similar fashion as is done in 141 for the Cartesian space:

$$
v=\min \left(1, \frac{\dot{q}_{\max }}{\sqrt{\dot{\boldsymbol{q}}_{\mathrm{sat}}^{\top} \boldsymbol{M}(\boldsymbol{q}) \dot{\boldsymbol{q}}_{\mathrm{sat}}}}\right) .
$$

The inner product in joint space is defined utilizing the mass matrix because a regular Euclidean norm is not physically meaningful [157]. The kinetic energy in the system is given by:

$$
E_{\mathrm{kin}}=\frac{1}{2} \dot{\boldsymbol{q}}^{\top} \boldsymbol{M}(\boldsymbol{q}) \dot{\boldsymbol{q}}
$$




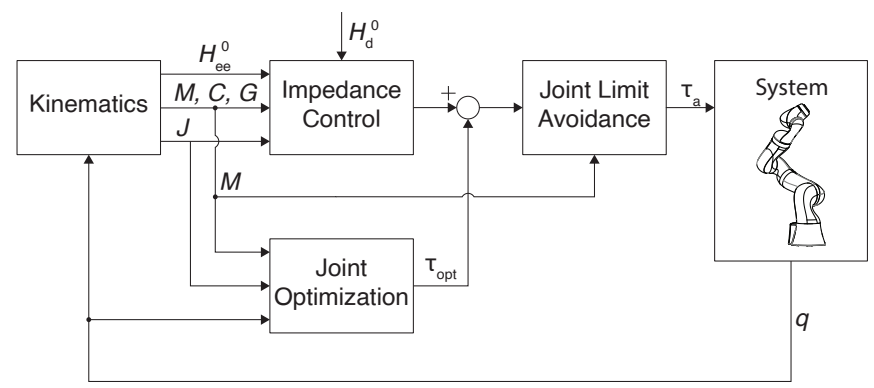

Figure 3.3: The resulting control scheme for a geometric impedance controller combined with our joint limit avoidance controller.

Thus, we can derive a value for $\dot{q}_{\max }$ that limits the kinetic energy due to the joint optimization to a set maximum kinetic energy, $E_{\max }$ :

$$
\dot{q}_{\max }=\sqrt{2 E_{\max }} .
$$

Finally, the torque that minimizes the potential energy and respects the null space is given by

$$
\boldsymbol{\tau}_{\mathrm{opt}}^{\top}=v \boldsymbol{\tau}_{0}-\boldsymbol{D}_{\mathrm{q}} \dot{\boldsymbol{q}}
$$

Also, motions not taking place in the null space are damped by this equation.

\subsubsection{Joint limit avoidance}

The joint position optimization allows the robot to centralize its joint positions given the null space constraints. However, reaching a limit is still possible. To prevent the robot from actually reaching any of its joint limits, a zone is defined in which the robot can safely operate, $-\beta_{i} q_{\lim _{i}} \leq q_{i} \leq \beta_{i} q_{\lim _{i}} \cdot \beta_{i}$ represents the fraction of the joint range that the robot can use. If a joint threatens to go out of this safe zone, then this joint faces an extra spring:

$$
\boldsymbol{\tau}_{\text {jla }_{i}}=\left\{\begin{array}{ll}
-\gamma_{i} \rho_{\mathrm{u}_{i}} & \text { if } \rho_{\mathrm{u}_{i}}+\dot{\rho}_{\mathrm{u}_{i}} T-\frac{1}{2} \ddot{q}_{\mathrm{a}_{i}} T^{2}<0 \\
\gamma_{i} \rho_{\mathrm{l}_{i}} & \text { if } \rho_{\mathrm{l}_{i}}+\dot{\rho}_{\mathrm{l}_{i}} T+\frac{1}{2} \ddot{q}_{\mathrm{a}_{i}} T^{2}<0 \\
0 & \text { other }
\end{array},\right.
$$

where $\gamma_{i}$ is the $i$-th component of the spring stiffness $\gamma \in \mathbb{R}^{7} \cdot \boldsymbol{\rho}_{\mathrm{u}}=\boldsymbol{\beta} \boldsymbol{q}_{\mathrm{lim}}-\boldsymbol{q}$, and $\boldsymbol{\rho}_{\mathrm{l}}=-\boldsymbol{\beta} \boldsymbol{q}_{\mathrm{lim}}-\boldsymbol{q}$ define the current distance to the upper and lower boundary of the previously defined safe range. $T$ is the duration of one control iteration and $\ddot{\boldsymbol{q}}_{\mathrm{a}}=\boldsymbol{M}^{-1} \boldsymbol{\tau}_{\mathrm{a}}$ is the expected acceleration if $\boldsymbol{\tau}_{\mathrm{jla}}$ would be zero. The joint position may be expected to leave the safe range the next control iteration based on the Taylor expansion of the current position, utilizing the current velocity and the expected acceleration due to the actuator torques. If, based on this 
estimation, the $i$-th joint is expected to leave the safe zone on either side of the range, $\tau_{\mathrm{jla}_{i}}$ is activated. Note that this approach is very similar to the one presented by Muñoz Osorio et al. [146. However, we implement a regular spring, because we already have a joint space damping. With a lower control rate and an imperfect model of the robot, this method is more suitable. A potential downside may be a small acceleration towards the spring in specific conditions.

The resulting control scheme is presented in Figure 3.3

\subsection{Experimental validation}

Experiments validated the controller in a simulation environment and on an actual robot.

\subsubsection{Experimental setup}

\section{Simulation environment}

The simulation is implemented in 20Sim (Controllab Products B.V., The Netherlands) utilizing the Matlab (The MathWorks Inc.) plugin. The robot is modeled using a bond graph approach similar to [158]. The Matlab Robotics Toolbox provides initial conditions for the simulation, such as the initial configuration and the null-space vector during simulations.

\section{Experimental setup}

The setup (Figure 3.4 consists of a 7-DOFs robotic manipulator (KUKA Med 7 R800, KUKA GmbH, Germany). This robot is connected to a workstation that runs the algorithm and communicates with the robot via the fast research interface with a $200 \mathrm{~Hz}$ update rate 159 .

\subsubsection{Experiments}

Two experiments were performed to evaluate the designed controller. Table 3.1 presents the settings unless they are explicitly varied during the experiment. $\boldsymbol{K}_{\mathrm{c}}$ is zero. The stiffness and damping parameters are empirically chosen using the simulated robot.

In the first experiment the robot was placed with the EE-frame at $(x, y, z)=$ $(-0.0575,0,0.9) \mathrm{m}$, the $\mathrm{x}$ - and the $\mathrm{z}$-axis aligned with the negative $\mathrm{z}$-axis and the $\mathrm{x}$-axis of the base, respectively (see Figure 3.4). The initial configuration has its joints positioned relatively close to their limits. Now, the controller was run for various values of $E_{\max }, E_{\max }=\left[\begin{array}{lllll}0 & 0.025 & 0.05 & 0.1 & 0.2\end{array}\right] \mathrm{J}$, to assess whether the robot respects the maximum kinetic energy and the current EE position, 


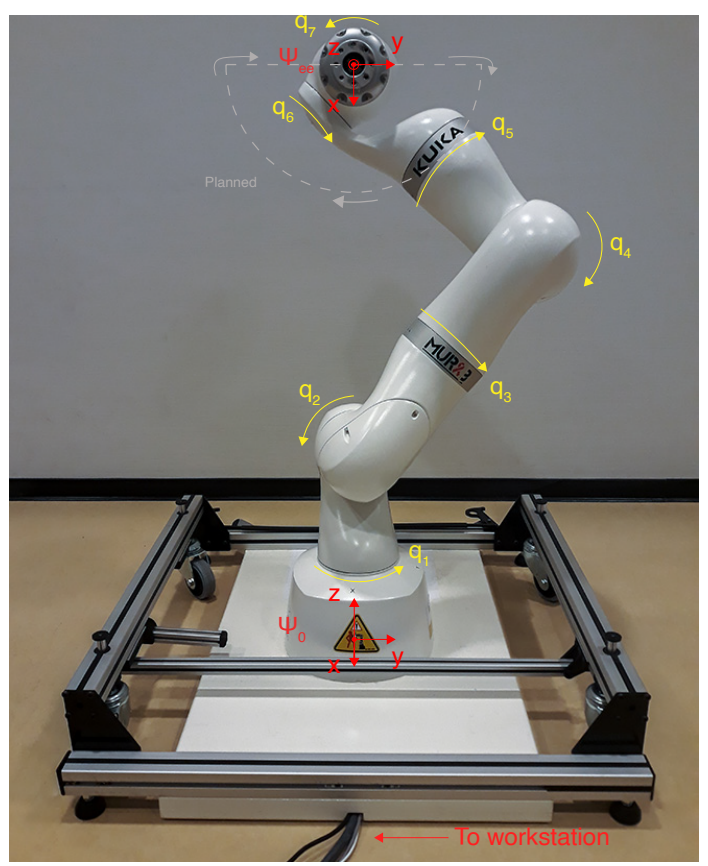

Figure 3.4: The setup to perform the measurements: A KUKA MED 7 R800 on a movable platform. Indicated are the base frame, $\Psi_{0}$, and the end-effector frame, $\Psi_{\text {ee }}$, the joints and the cables for communication with the work station. The dashed gray line represents the planned path in the trajectory tracking experiment.

and whether the robot navigates to an energetic minimum. These steps were performed both in simulation and on the actual robot.

Next, the actual robot performs the trajectory indicated in Figure 3.4. The robot cannot perform this trajectory accurately since the bottom of the halfcircle is outside the work space, and joint four will reach its limit. Therefore, this trajectory will activate the controller's joint limit avoidance behavior. By performing the trajectory for $E_{\max }=\left[\begin{array}{ll}0 & 0.2\end{array}\right] \mathrm{J}$, we see how the trajectory tracking is affected by the null space impedance and whether the average joint position is minimized during trajectory tracking.

\subsubsection{Results}

Figure 3.5 presents the results of the first experiment. Figure 3.5(a) shows the system's kinetic energy over time. It shows that the simulated and the experimental behavior are very similar. However, in the experiments, the kinetic energy is always below the allowed kinetic energy, $E_{\max }$, because of joint frictions which are present in the joints. These frictions are not present in the 
simulated robot, and as such, the kinetic energy is closer to the setpoint in the simulations. Additionally, the experimental behavior shows some overshoot, even for $E_{\max }=0 \mathrm{~J}$, because the gravity compensation on the actual robot is not perfect, and the robot drops several millimeters at the start of the experiment.

This drop is also present in Figure 3.5(b), which shows the Euclidean error over time. The Euclidean error, defined as $e=\sqrt{e_{x}^{2}+e_{y}^{2}+e_{z}^{2}}$, is around $2 \mathrm{~mm}$ for $E_{\max }=0 \mathrm{~J}$. The other experiments show a maximum error of around $5 \mathrm{~mm}$. The positional accuracy could be improved by increasing the stiffness parameters but is limited by, e.g., joint friction, imperfect gravity compensation and the control rate. Even though these imperfections are not present in the simulation, it shows sub-millimeter errors. The joints in the physical model are not infinitely stiff, as this increases the simulation time. Thus, large accelerations and forces result in small Euclidean errors.

Finally, Figure 3.5 (c) shows the residual gradient of the potential field along the null-space vector. As expected, with $E_{\max }=0 \mathrm{~J}$, the initial gradient of $1.36 \times 10^{4} \mathrm{~N} \mathrm{~m}$ does not change much during an experiment/simulation. In the simulation environment, the residual gradient will reach values close to zero for $E_{\max }>0 \mathrm{~J}$. However, on the actual robot, the residual gradient is stuck at values around $7 \mathrm{Nm}$. This effect is also attributed to imperfections such as joint frictions.

In Figure 3.6 the performance of the controller during trajectory tracking is presented. In Figure 3.6(a), the planned trajectory is plotted together with the actual trajectories for $E_{\max }=0 \mathrm{~J}$ and $E_{\max }=0.2 \mathrm{~J}$. It shows that indeed the bottom part of the planned trajectory is not reached. The controller performs similarly for the two settings of the null-space impedance. Imperfections in the trajectory are similar for both values of $E_{\max }$. The robot cuts the left corner as $\boldsymbol{H}_{\mathrm{d}}^{0}$ is too far ahead. Additionally, the horizontal section is affected by position-dependent imperfections in the gravity compensation. In Figure 3.6. (b). it is shown that the average of the joint position vector, $\boldsymbol{q}$, is lower when the null-space spring is activated, which is as expected. The dip, which is present around $18 \mathrm{~s}$, is due to the elbow of the robot changing sides at the lowest point

Table 3.1: The values of the various variables during simulations and experiments.

\begin{tabular}{lrll}
\hline Variable & Value & Unit \\
\cline { 1 - 1 } $\boldsymbol{K}_{\mathrm{o}}$ & $100 \boldsymbol{I}^{3 \times 3}$ & & $\mathrm{~N} \mathrm{~m}^{-1}$ \\
$\boldsymbol{K}_{\mathrm{t}}$ & $1000 \boldsymbol{I}^{3 \times 3}$ & & $\mathrm{~N} \mathrm{~m} \mathrm{rad}^{-1}$ \\
$\boldsymbol{D}_{\mathrm{q}}$ & $\boldsymbol{M}(\boldsymbol{q}) 40 \boldsymbol{I}^{7 \times 7}$ & & $\mathrm{~N} \mathrm{~m} \mathrm{~s} \mathrm{rad}^{-1}$ \\
{$\left[\alpha_{1} \ldots \alpha_{7}\right]$} & 60 & - \\
{$\left[\beta_{1} \ldots \beta_{7}\right]$} & $\beta_{i} q_{\lim _{i}}=q_{\lim _{i}}-\frac{7^{\circ}}{180^{\circ}} \pi$ & $\mathrm{rad}$ \\
{$\left[\gamma_{1} \ldots \gamma_{7}\right]$} & 2000 & $\mathrm{~N} \mathrm{\text {rad}^{-1 }}$ \\
\hline
\end{tabular}


of the trajectory. Figure 3.6(c) presents the position of joint four, which was expected to reach the boundary of the safe zone. There is little difference between both experiments since joint four plays a crucial role in keeping the current EE configuration; hence, the optimization will not affect joint four. In both experiments, the joint position reached the boundary but did not go much past $-\beta_{4} q_{4}$, which is due to the joint limit avoidance torques.

\subsection{Discussion}

This work describes the formulation and implementation of a controller that compliantly optimizes its range of motion and avoids joint limits. Its compliance makes the controller ideal for an interactive and uncertain environment. The controller depends on a virtual Cartesian equilibrium position as well as a virtual joint space equilibrium. The Cartesian equilibrium is independent of the joint space equilibrium, even if they are incompatible. Instead, the controller finds a local minimum in joint space while respecting the EE configuration. Additionally, joint constraints are respected if the current Cartesian position is unreachable, starting from the current robot configuration.

Even though the presented controller performs similarly to controllers that project the force in the null space utilizing a projection matrix, it has some clear advantages. Methods that use the pseudo-inverse may perform poorly near the boundaries of the workspace, whereas the null space vector always exists, even if it is a zero vector. This paper only discusses one redundant DOF, but if more DOFs are available, a linear combination of the null space vectors may determine in which way the energy is released. An extra benefit of our controller is the dynamic shaping of the spring stiffness. This enables the use of nonlinear springs, and thus, user-defined prioritization of motions away from joints that are currently closer to their limits. There are various ways of storage and discharge of virtual energy that can still be explored: robots that need to move around a known obstacle may change the stiffness of individual springs to prefer bending a particular joint or set of joints. Additionally, the setpoints of the springs could be changed dynamically. However, the nonlinear springs we implemented are most suitable for the symmetric case. If other setpoints of the joint positions and asymmetric spring stiffnesses are necessary, the FIRAS function used by Khatib [143] may offer more flexibility.

Currently, the kinetic energy setpoint only bounds the release of energy in the null space, so this neglects motions generated by the Cartesian spring. Alternatively, the maximum allowable null space kinetic energy could be a function of the total acceptable kinetic energy and the kinetic energy due to the spring in the work space. For this, the work presented by Raiola et al. $\sqrt{160}$ could be a good starting point .

The controller does not explicitly allow task stacking and prioritizing as in 


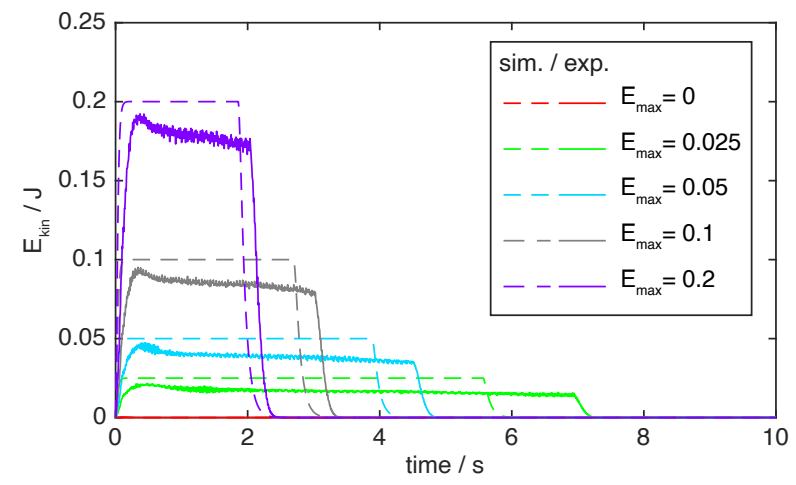

(a)

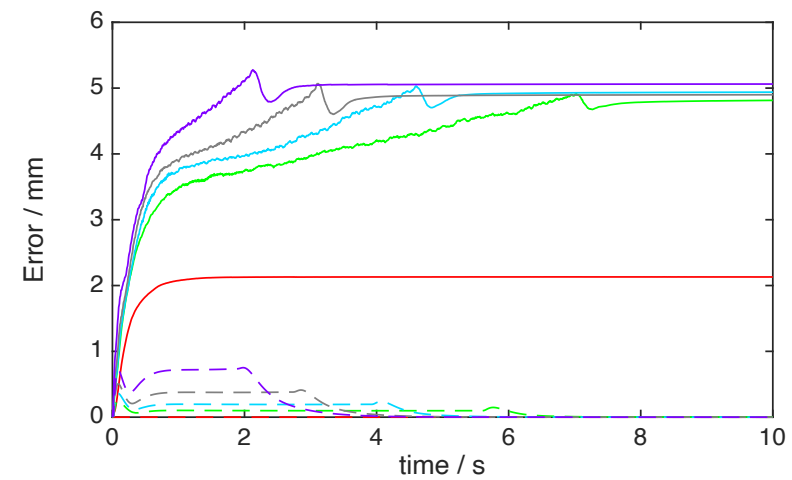

(b)

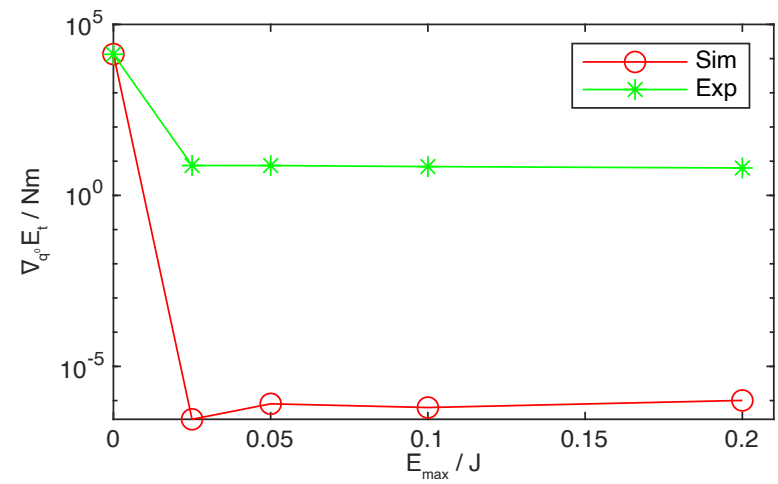

(c)

Figure 3.5: (a) Kinetic energy over time for different setpoints of $E_{\max }$ in simulation (dashed line) and on the actual robot (continuous line). (b) Euclidean error during the simulations and experiments. (c) Residual gradient as a function of $E_{\max }$ for the simulations and the experiments. 


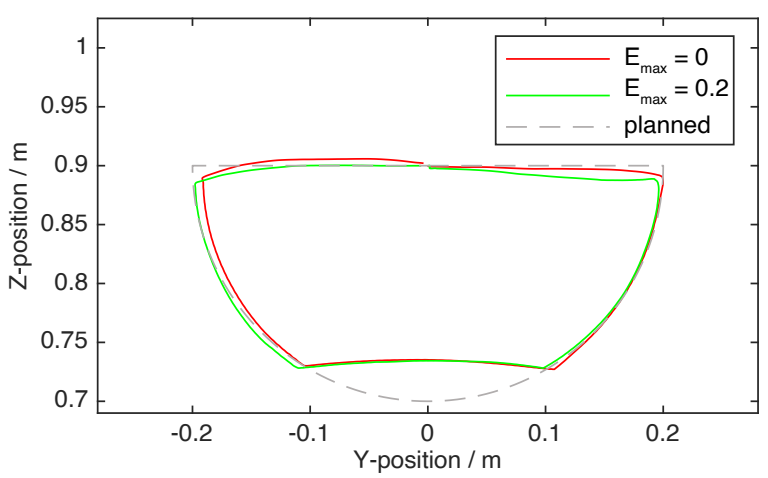

(a)

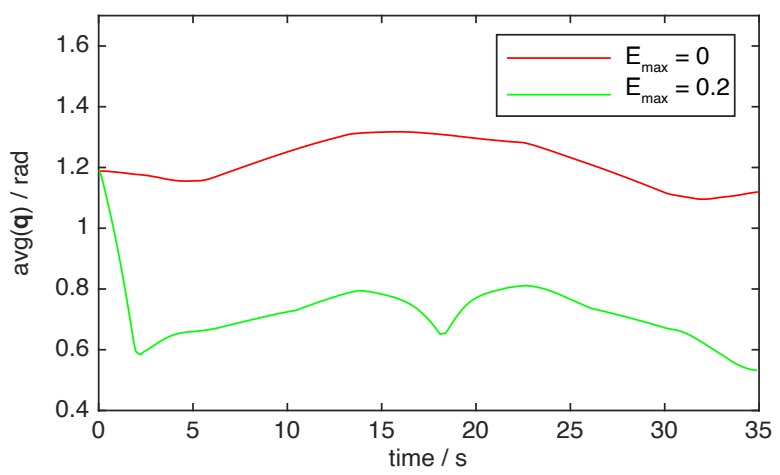

(b)

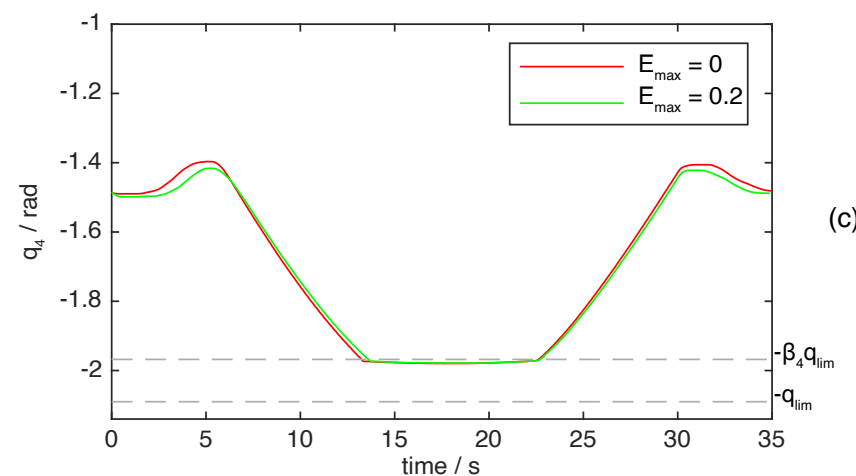

Figure 3.6: (a) Planned versus experimentally obtained trajectory of the EE for different values of $E_{\max }$. (b) Average joint position over time during trajectory tracking. (c) Joint limit avoidance behavior of joint four, which reached the edge of the defined safe zone. 
Muñoz Osorio et al. 146. Therefore, it is likely that their controller performs specific tasks more accurately while respecting the limits. However, our controller does not show any oscillations either, and the lower gain makes it more suitable for systems controlled at a lower rate. Several impedance controllers can be placed in series to achieve more tasks simultaneously. Thus, hard limits such as obstacles and self-collisions can still be implemented, as done in, e.g., 145].

\subsection{Conclusion}

We introduced a compliant pose optimization controller which functions alongside a common geometric impedance controller. We showed that the kinetic energy is bounded by dynamically changing the potential field and that the motion takes place in the null space of the manipulator. Additionally, we showed that indeed the robot moves towards a local minimum in the potential field. Finally, we show that trajectory tracking is minimally influenced. The robot both optimizes its configuration by minimizing the magnitude of the vector of joint positions and actively prevents the robot from reaching a limit. 


\section{$4 \quad$ Automated robotic breast ultrasound acquisition using ultrasound feedback}

Adapted from:

M. K. Welleweerd, A. G. de Groot, S. O. H. de Looijer, F. J. Siepel, and S. Stramigioli, "Automated robotic breast ultrasound acquisition using ultrasound feedback," in 2020 IEEE International Conference on Robotics and Automation (ICRA), IEEE, May 2020, pp. 9946-9952, ISBN: 978-1-7281-7395-5. DOI: 10 1109/ICRA40945.2020.9196736

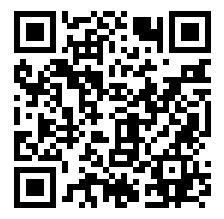




\begin{abstract}
Current challenges in automated robotic breast ultrasound (US) acquisitions include keeping acoustic coupling between the breast and the US probe, minimizing tissue deformations and safety. This paper presents how an autonomous 3D breast US acquisition can be performed utilizing a seven-degrees-of-freedom robot equipped with a linear US transducer. Robotic 3D breast US acquisitions would increase the diagnostic value of the modality since they allow patientspecific scans and have high reproducibility, accuracy and efficiency. Additionally, 3D US acquisitions allow more flexibility in examining the breast and simplify registration with preoperative images like magnetic resonance imaging (MRI). In the presented approach, the robot follows a reference-based trajectory adjusted by a visual servoing algorithm. The reference trajectory is a patient-specific trajectory coming from, e.g., MRI. The visual servoing algorithm commands in-plane rotations and corrects the probe contact based on confidence maps. A safety-aware, intrinsically-passive framework is utilized to actuate the robot. The approach is illustrated with experiments on a phantom, which show that the robot only needs minor pre-procedural information to image the phantom consistently while relying primarily on US feedback
\end{abstract}




\subsection{Introduction}

Ultrasound (US) imaging has become an essential diagnostic tool for breast cancer detection. It is used to localize the lesion and confirm the pathology in the diagnostic biopsy procedure. US has advantages over other imaging methods because it is cheap, safe, and can display images of the region of interest (ROI) in real-time. Furthermore, it is more reliable than mammography in detecting cancers in dense breasted women and distinguishes between cystic and solid lesions. Also, malignant lesions are recognized with $98 \%$ confidence [161.

However, the use of handheld US has substantial limitations as well. The accuracy of probe manipulation is highly operator-dependent, the reproducibility is low, and the procedure is time-consuming. It is challenging to measure structures of interest reliably because the images represent $2 \mathrm{D}$ cross-sections. Finally, relating current images to 3D preoperative images during, e.g., USguided biopsies on magnetic resonance (MR)-detected lesions is complex due to the lack of discernible spatial features.

3D US breast acquisitions would overcome these limitations: larger ROIs can be imaged, arbitrary cross-sections of this ROI are possible, and more precise measurements of the size and volume of lesions are possible. Moreover, it is easier to register preoperative data like an magnetic resonance imaging (MRI) of the breast with the intraoperative US data because a volume contains more features than a $2 \mathrm{D}$ image.

Currently, there are several approaches to acquiring 3D US breast data. Manual acquisitions can be divided into freehand scanning, mechanical scanning and 2D array scanning [162]. Automated scanners include contactless scanners which scan the patient in prone position, with the breast submerged in a liquid. Other scanners scan the patient in supine position, the probe being in contact with the breast 163. Both automated systems have disadvantages: either part of the signal is lost due to the US traveling through the liquid, or volume reconstruction is complex due to deformation of the breast.

Compared to the conventional approaches, the advantages of utilizing a robotically manipulated linear US probe for automated breast volume acquisitions are plentiful. Robotic arms offer higher accuracy than other tracked systems and enhanced dexterity and consistency than human operators. The linear US probes are widespread in medical imaging and offer a higher resolution than 3D US probes. Thus, a robotic manipulator with a US probe can acquire highquality, accurately localized slices. In addition, acquisitions can be tailored to the individuals' breast shape, possibly making US volume reconstructions more accurate and efficient. Robotics has already shown its potential in several other medical US applications 95, 164, 165.

Nevertheless, there are also some challenges in automated robotic US breast acquisitions. The breast is highly deformable, so there is a balance between acoustic coupling and applied pressure. A deformed acquisition is more difficult 
to reconstruct and register with other modalities. Another aspect is the safety of both the patient and the system operator.

Keeping contact can be achieved in several ways. In static situations, a trajectory closely describing skin surface can be generated utilizing preoperative images such as MRI and registering the trajectory with the patient in robot coordinates. Trajectories can also be generated in real-time by reconstructing the skin surface using commercially available $3 \mathrm{D}$ cameras $[163$. However, having a predefined trajectory does not guarantee probe contact since involuntary patient movements or measurement inaccuracies may occur. Therefore, several studies implemented force control strategies in their application [69, 73, 166 168. While normal force is closely related to acoustic coupling, it is not solely responsible for high-quality images. Scanning with force feedback may unnecessarily deform a softer breast, while the image quality may decline when scanning a stiffer breast. Visual servoing algorithms link end-effector (EE) behavior to image features. In [75] and [76], an intensity based method is proposed to control the probe. Additionally, in-plane motions can be controlled using feature tracking [77], while out-of-plane motions can be controlled by image moments [78, speckle correlation $[79]$ or block matching 169 . Recently, confidence maps have been successfully implemented by 69,166 168. A confidence map represents the confidence in the US signal in a pixel-wise manner. It has been used to avoid imaging shadowing objects, achieve uniform probe contact, and optimize a target region or the global image.

Safety is paramount in medical robotics since the robots operate in an unstructured environment and interact with patients. Therefore, compliance of the robotic arm should be introduced to account for uncontrolled impact and patient movements. Several safety metrics have been suggested, such as Head Impact Power and Head Injury Criteria, and safety-based controllers have been implemented limiting the robot's velocity, force, power, and energy [160].

This study aims to perform a breast shape-preserving, safe automated robotic 3D US volume acquisition of the breast. High-quality, patient-specific acquisitions, obtained while considering safety, are necessary to bring applications like this closer to clinical practice. We developed a setup to achieve this. The patient is positioned in prone position, and an initial scanning trajectory is extracted from the preoperative MRI. This scanning trajectory may not be accurate due to deformations during repositioning of the patient or involuntary movements. Thus, feedback is essential for autonomous scanning. Since force feedback may have varying results over different breasts, we propose a system solely relying on US feedback. Confidence maps are utilized to maintain the contact between the probe and the breast and minimize the applied pressure. We implemented the trajectory following and visual servoing adjustments in a safety-aware, intrinsically-passive (SAIP) control framework to ensure the patient's safety. The performed experiments show that the robot consistently images a complex scanning trajectory based on minimal pre-procedural input. 

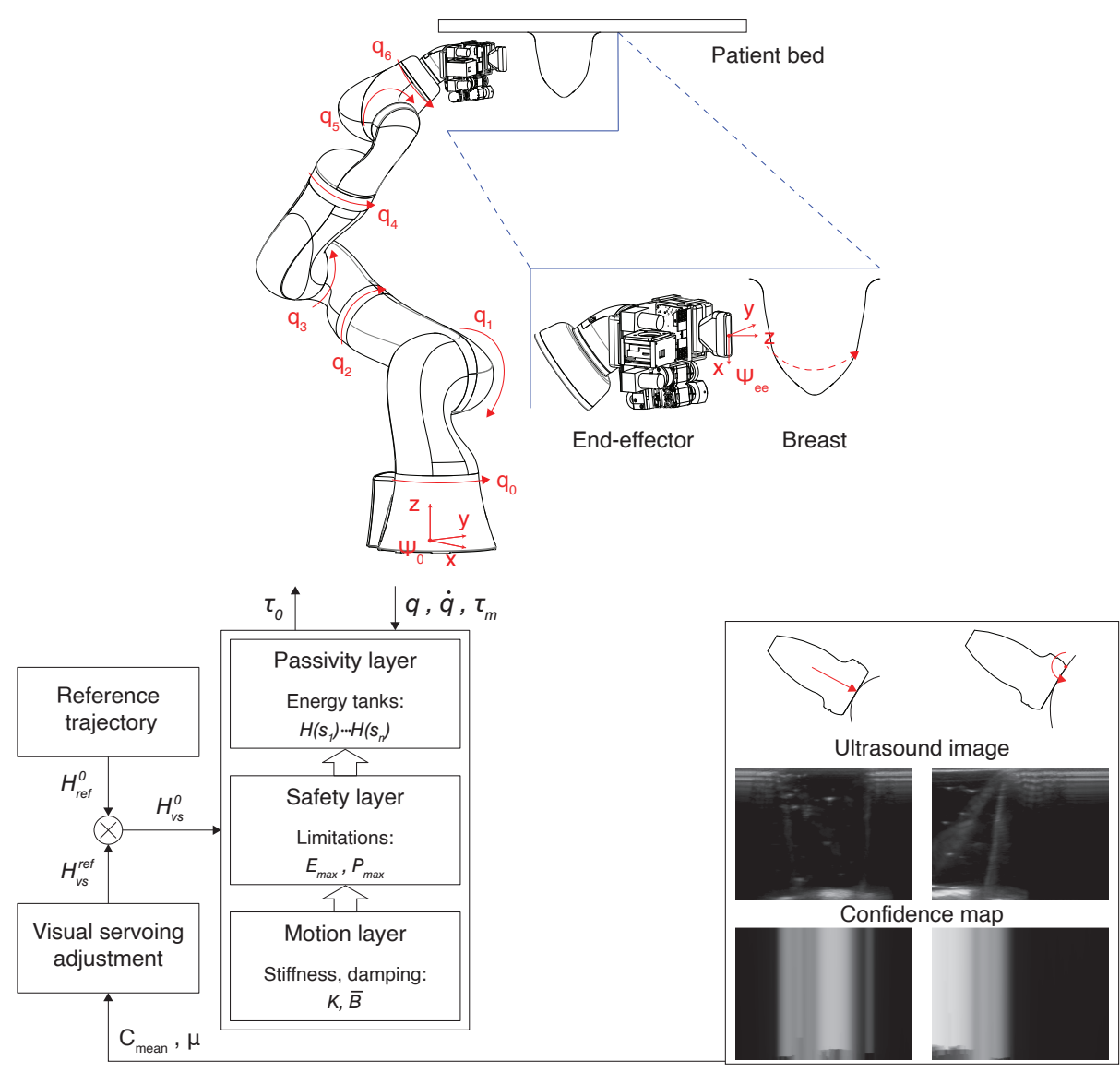

Figure 4.1: A system overview. The robot is placed underneath the breast. Scanning is done based on a reference trajectory adjusted with visual servoing information. A safety-aware, intrinsically-passive controller actuates the robot.

\subsection{Automated robotic breast ultrasound}

\subsubsection{System overview}

Figure 4.1 presents a system overview. The patient lies on a patient bed in prone position with the examined breast through a hole such that it is freely accessible for the robot. The robot is placed underneath the bed. It is equipped with an EE holding a linear US transducer [170]. The robot follows a trajectory over the breast surface with the US transducer to acquire a series of $2 \mathrm{D}$ US images for 


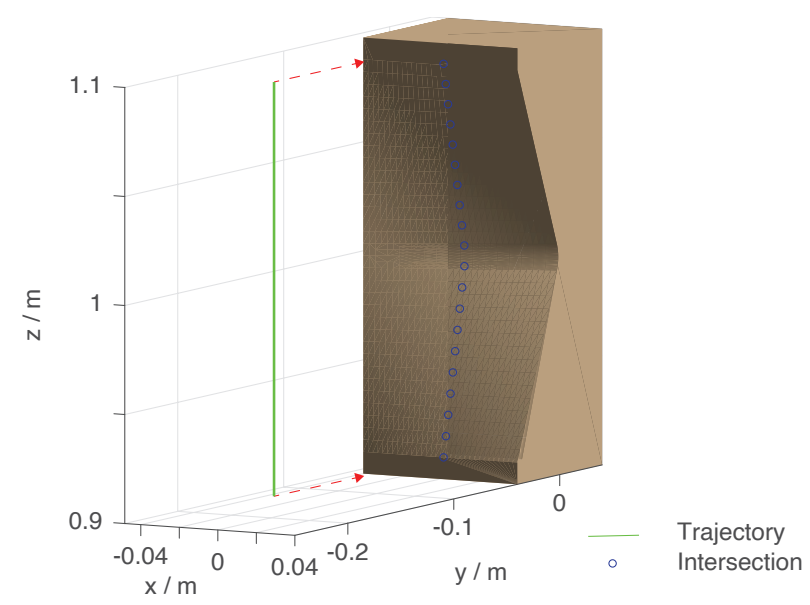

Figure 4.2: Tesselated phantom used for the experiments with in green the projected trajectory and in blue the intersections.

$3 \mathrm{D}$ volume reconstruction. This trajectory is based on preoperative images such as MRI adapted with real-time visual servoing input coming from US confidence maps. This trajectory is the input for a SAIP control algorithm that actuates the robot. The different parts of the robot control are described in the following subsections.

\subsubsection{Path planning}

The path is defined as a series of homogeneous matrices $H_{\mathrm{ref}}^{0}(i)$ located on the patient's skin, specifying the desired poses and positions of the transducer in the robot base frame. A patient's or phantom's surface is obtained by converting the MRI scan or the computer-aided design (CAD) file to a tessellated surface reconstruction. The waypoints are generated by casting rays from a predefined path like a line or spiral towards the surface (see Figure 4.2). The intersection point of the ray with the surface is calculated utilizing the Fast, Minimum Storage Ray/Triangle intersection [171]. These intersection points represent the translation of the EE. The desired orientation of the EE is normal to the skin in each position. This normal is extracted from the intersected triangle utilizing the vertices. The x-axis' orientation is obtained by the cross-product of the z-axis and the unit-vector in the next point's direction. The y-axis is obtained by taking the cross-product of the z-axis with the x-axis. The correct transformation of the waypoints relative to the base frame is acquired by manual measurement or marker localization. 


\subsubsection{Confidence maps}

Confidence maps provide a per-pixel measure of uncertainty of the acquired US image. Each US image $I: \Omega \rightarrow[0,1]$ is associated with a confidence map $C: \Omega \rightarrow[0,1]$ in which each pixel $(i, j) \in \Omega:=[1 . . n] \times[1 . . m]$ represents the confidence of that pixel in the US image $I(i, j)$. The US image, $I$, is mapped to the confidence map, $C$, based on the probability of a random walk starting from a pixel $(i, j)$ and reaching each of the virtual transducer elements 172]. The random walks algorithm meets three constraints: the top row of the US image has confidence 1 , the bottom row has confidence 0 , and the signal obeys USspecific propagation constraints 167]. Confidence maps accentuate attenuated and shadowed parts of an image. As such, they are useful to estimate how the probe is in contact with the skin.

\subsubsection{Control strategy}

The robot should move the US probe over the patient's skin without losing contact or causing too much compression. The general shape of the patient's body has been extracted from the MRI and localized relative to the robot. However, small deformations or involuntary movements of the patient may cause the actual trajectory to differ from the calculated trajectory. Thus, the probe may inadvertently lose contact with the patient, press too hard, or partly contact the skin. The confidence maps can be used to control two degrees of freedom of the probe: the in-plane rotation and the translation in the z-direction of the EE frame, $\Psi_{\text {ee }}$, as defined in Figure 4.1. The predefined path determines the other four degrees of freedom. Thus, the reference pose $\boldsymbol{H}_{\mathrm{ref}}^{0}(i)$ of the probe at time $i$ is adjusted by $\boldsymbol{H}(i)_{\text {adj }}^{\text {ref }}$ according to:

$$
\begin{aligned}
\boldsymbol{H}(i)_{\mathrm{vs}}^{0} & =\boldsymbol{H}(i)_{\mathrm{ref}}^{0} \boldsymbol{H}(i)_{\mathrm{adj}}^{\mathrm{ref}} \\
& =\boldsymbol{H}(i)_{\mathrm{ref}}^{0} \boldsymbol{H}(i-1)_{\mathrm{adj}}^{\mathrm{ref}} \boldsymbol{H}(i)_{\mathrm{vs}}^{\mathrm{adj}}, \\
\boldsymbol{H}(i)_{\mathrm{vs}}^{\mathrm{adj}} & =\boldsymbol{H}_{\Delta \mathrm{z}} \boldsymbol{H}_{\mathrm{t}} \boldsymbol{H}_{\Delta \theta} .
\end{aligned}
$$

$\boldsymbol{H}(i)_{\text {adj }}^{\text {ref }}$ is composed of the current and previous outputs of the visual servoing algorithm, $\boldsymbol{H}_{\mathrm{vs}}^{\text {adj }}$ and $\boldsymbol{H}(i-1)_{\mathrm{adj}}^{\mathrm{ref}}$, respectively. The visual servoing algorithm outputs a translation, $\Delta z$, in z-direction, $\boldsymbol{H}_{\Delta \mathrm{z}}$, and a rotation, $\Delta \theta$, around either end of the transducer, $\boldsymbol{H}_{\mathrm{t}} \boldsymbol{H}_{\Delta \theta} \cdot \boldsymbol{H}_{t}$ helps expressing the rotation in the correct frame by a transformation of half the probe-width along the x-axis of the transducer.

Two features are extracted from the confidence maps that indicate whether rotation or translation in the z-direction is needed to improve the contact of the US probe with the skin. Only the top section, $P \subset \Omega$, of the confidence map is used for feature extraction to make these features independent of the patient's 
physiology. This section of the female breast consists of skin and fatty tissue, while further from the surface, muscle tissue and ribs are located, which are not US transparent.

As shown in Figure 4.1 if the probe is in contact with the skin on one side, the confidence is unevenly spread over the image. The confidence weighted barycentre, $\mu$, of the confidence map gives a measure of how the confidence is divided over the image and is defined as

$$
\begin{aligned}
\mu_{i} & =\frac{1}{C_{\mathrm{P}}} \sum_{(i, j) \in P} i \cdot C(i, j), \\
\mu_{j} & =\frac{1}{C_{\mathrm{P}}} \sum_{(i, j) \in P} j \cdot C(i, j),
\end{aligned}
$$

with $C_{\mathrm{P}}=\sum_{(i, j) \in} C(i, j)$ the total confidence. The pixel indices $\mu_{i}$ and $\mu_{j}$ are converted to coordinates in the EE frame, $\mu_{z}$ and $\mu_{x}$, respectively. The desired $\mathrm{x}$-coordinate of the barycentre is around 0 , as this indicates the probe is oriented normal to the skin. The angle between the central scan line $(x=0)$ and the line passing through the origin and the barycentre is a measure for the error between the current and the desired pose of the probe $e_{\theta}=\arctan \frac{\mu_{x}}{\mu_{z}}$.

The mean confidence $C_{\text {mean }}$ of a US image indicates which portion of the transducer area is in contact with the skin as the breast is a curved surface:

$$
C_{\text {mean }}=\frac{1}{m \cdot n} \sum_{(i, j) \in P} C(i, j) .
$$

The error is defined by the set mean, $C_{\text {set }}$, and the currently measured mean confidence, $C_{\text {mean }}$ : $e_{\mathrm{c}}=C_{\text {set }}-C_{\text {mean }}$.

A simple PD controller controls the confidence weighted barycentre and the mean confidence. The error is scaled with a third-order function to give large errors more weight than small ones. For safety reasons, the maximum deviation of the visual servoing algorithm with respect to the original reference path is limited.

\subsubsection{Safety-aware intrinsically-passive controller}

The control architecture of the robot is implemented according to the SAIP control scheme presented in 160 . Impedance control is practical in humanrobot interaction because environmental uncertainty requires compliant robotic behavior. Safe human-robot interaction can be warranted by monitoring the energy and power output of the robot. Passivity is a fundamental criterion of a system because if passivity is not preserved, the possibility exists that a passive environment destabilizes the robot 173 . The definition of a passive system is a stable dynamic system of which the total energy is never higher than the sum of its initial energy and any external energy supplied to it by interaction. 


\section{Impedance control}

For a detailed overview of the implementation of the controller, please refer to [160. The dynamic equation of the controlled system is given by

$$
\boldsymbol{M}(\boldsymbol{q}) \ddot{\boldsymbol{q}}+\boldsymbol{C}(\boldsymbol{q}, \dot{\boldsymbol{q}}) \dot{\boldsymbol{q}}+\boldsymbol{F}(\boldsymbol{q}, \dot{\boldsymbol{q}})^{\top}+\boldsymbol{G}(\boldsymbol{q})^{\top}=\underbrace{\boldsymbol{J}^{\top}(\boldsymbol{q}) \boldsymbol{W}^{0^{\top}}}_{\text {elastic wrench }}-\boldsymbol{B} \dot{\boldsymbol{q}}+\underbrace{\boldsymbol{J}^{\top}(\boldsymbol{q}) \boldsymbol{W}^{0^{\top}}}_{\text {external wrench }}
$$

where $\boldsymbol{J}(\boldsymbol{q})$ is the manipulator jacobian, $\boldsymbol{M}(\boldsymbol{q})$ is the inertia matrix, $\boldsymbol{C}(\boldsymbol{q}, \dot{\boldsymbol{q}})$ represents the Coriolis and centrifugal terms, $\boldsymbol{F}(\boldsymbol{q}, \dot{\boldsymbol{q}})$ contains the friction forces, $\boldsymbol{G}(\boldsymbol{q})$ is the gravitational term, $\boldsymbol{B}(\boldsymbol{q})$ represents the damping and $\boldsymbol{q}$ is the vector with joint positions. The elastic wrench and the external wrench are wrenches applied to the EE induced by the virtual spring and external forces, respectively. The control law for the actuator torques, $\tau_{\mathrm{a}}$, then becomes:

$$
\boldsymbol{\tau}_{\mathrm{a}}^{\top}=\boldsymbol{J}^{\top}(\boldsymbol{q}) \boldsymbol{W}^{0^{\top}}-\boldsymbol{B}(\boldsymbol{q}) \dot{\boldsymbol{q}}+\boldsymbol{J}^{\top}(\boldsymbol{q}) \boldsymbol{W}^{0^{\top}}-\hat{\boldsymbol{C}}(\boldsymbol{q}, \dot{\boldsymbol{q}}) \dot{\boldsymbol{q}}-\hat{\boldsymbol{F}}(\boldsymbol{q}, \dot{\boldsymbol{q}})^{\top}-\hat{\boldsymbol{G}}(\boldsymbol{q})^{\top}
$$

$\hat{\boldsymbol{C}}(\boldsymbol{q}, \dot{\boldsymbol{q}}), \hat{\boldsymbol{F}}(\boldsymbol{q}, \dot{\boldsymbol{q}})$, and $\hat{\boldsymbol{G}}(\boldsymbol{q})$ are compensation terms for Coriolis and centrifugal forces, for friction forces and gravity forces respectively.

\section{Safety-aware impedance control}

Both energy and power should be monitored and limited to guarantee the safety of the robot's interaction. The total energy, $E_{\text {tot }}$, of the robot is defined by the kinetic energy and the potential energy stored in the virtual spring between the current and desired position such that

$$
E_{\mathrm{tot}}=T(\boldsymbol{q}, \dot{\boldsymbol{q}})+V\left(\boldsymbol{R}_{\mathrm{c}}^{\mathrm{d}}, \boldsymbol{p}_{\mathrm{c}}^{\mathrm{d}}\right),
$$

in which $T(\boldsymbol{q}, \dot{\boldsymbol{q}})$ is the kinetic energy of the links, $V\left(\boldsymbol{R}_{\mathrm{c}}^{\mathrm{d}}, \boldsymbol{p}_{\mathrm{c}}^{\mathrm{d}}\right)$ is the potential energy in the virtual spring and $R_{\mathrm{c}}^{\mathrm{d}}$ and $p_{\mathrm{c}}^{\mathrm{d}}$ are respectively the rotation and translation between the current and desired EE position. Energy limitation of $E_{\text {tot }}$ is achieved by modifying the stiffness of the virtual spring and thus the potential energy. A scaling factor, $\lambda$, is defined, which scales the stiffness matrices to limit the potential energy:

$$
\lambda=\left\{\begin{array}{ll}
1 & \text { for } E_{\mathrm{tot}} \leq E_{\max } \\
\frac{E_{\max }-T(\boldsymbol{q}, \boldsymbol{q})}{V\left(\boldsymbol{R}_{\mathrm{c}}^{\mathrm{d}}, \boldsymbol{p}_{\mathrm{c}}^{\mathrm{d}}\right)} & \text { for } E_{\mathrm{tot}}>E_{\max }
\end{array} .\right.
$$

Because the potential energy scales linearly with the stiffness of the virtual spring. The total energy of the system becomes

$$
E_{\mathrm{tot}}=T(\boldsymbol{q}, \dot{\boldsymbol{q}})+\lambda V\left(\boldsymbol{R}_{\mathrm{c}}^{\mathrm{d}}, \boldsymbol{p}_{\mathrm{c}}^{\mathrm{d}}\right) .
$$


The total power flow $P_{\mathrm{c}}$ from the controller to the robot is defined by the power $P_{\mathrm{m}}$ for motions of the robot and the power $P_{\mathrm{g}}$ consumed for compensating the gravity and keeping the robot in its current configuration:

$$
P_{\mathrm{c}}=\underbrace{\left(\boldsymbol{J}^{\top}(\boldsymbol{q}) \boldsymbol{W}^{0^{\top}}-\boldsymbol{B}(\boldsymbol{q}) \dot{\boldsymbol{q}}\right)^{\top} \dot{\boldsymbol{q}}}_{P_{\mathrm{m}}}+\underbrace{\hat{\boldsymbol{G}}(\boldsymbol{q}) \dot{\boldsymbol{q}}}_{P_{\mathrm{g}}} .
$$

$P_{\mathrm{m}}$ represents the power that can be transferred to the environment while $P_{\mathrm{g}}$ is used by the robot itself to compensate its weight. Thus, $P_{\mathrm{m}}$ should be limited to a value $P_{\max }$ to prevent injuries. This is done by adding a scaling factor, $\beta$, to the damping:

$$
\beta=\left\{\begin{array}{lll}
1 & \text { for } \quad P_{\mathrm{c}} \leq P_{\max } \\
\frac{\boldsymbol{J}^{\top}(\boldsymbol{q}) \boldsymbol{W}^{0^{\top}} \dot{\boldsymbol{q}}-P_{\max }}{\dot{\boldsymbol{q}}^{\top} \boldsymbol{B}(\boldsymbol{q}) \dot{\boldsymbol{q}}} & \text { for } \quad P_{\mathrm{c}}>P_{\max }
\end{array} .\right.
$$

The resulting power of the system is

$$
P_{\mathrm{c}}=\left(\boldsymbol{J}^{\top}(\boldsymbol{q}) \boldsymbol{W}^{0^{\top}}-\beta \boldsymbol{B}(\boldsymbol{q}) \dot{\boldsymbol{q}}\right)^{\top} \dot{\boldsymbol{q}}+\hat{\boldsymbol{G}}(\boldsymbol{q}) \dot{\boldsymbol{q}}
$$

To summarize, the energy in the system and the power flow from the controller to the robot are monitored by the controller, which scales the stiffness and damping to ensure that both stay within limits.

\section{Assuring passivity}

Passivity is assured by adding an energy tank to each joint. For each joint, the energy tank $H(s)$ is modeled as a spring with a constant stiffness such that the energy in the tank is defined as $H(s)=\frac{1}{2} k s^{2}$. The spring is connected to the joint via a transmission ratio $u_{n}$ such that the port-Hamiltonian formulation becomes

$$
\left(\begin{array}{c}
\dot{s}_{n} \\
\tau_{\mathrm{o}_{n}}
\end{array}\right)=\left(\begin{array}{cc}
0 & u_{n} \\
-u_{n} & 0
\end{array}\right)\left(\begin{array}{c}
s_{n} \\
\dot{q}_{n}
\end{array}\right),
$$

in which $s_{n}$ is the spring state of joint $n, \dot{q}$ is the joint velocity, $\tau_{\mathrm{o}_{n}}$ is the torque at the output and $u_{n}$ is defined as

$$
u_{n}=\left\{\begin{array}{ll}
\frac{-\tau_{c_{n}}}{s_{n}} & \text { for } H_{n}\left(s_{n}\right)>\epsilon \\
\frac{-\tau_{c_{n}}}{\gamma^{2}} s_{n} & \text { otherwise }
\end{array},\right.
$$

where $\gamma=\sqrt{2 \epsilon}, \tau_{\mathrm{c}_{n}}$ is the torque computed by the safety-aware controller and $\epsilon$ is a threshold value which indicates a nearly empty tank. When this value is reached, the transmission ratio does not become zero. This way, a control action still takes place and prevents recharging of the tank due to the inertial motion of the other links. The torque output sent to joint $n$ is

$$
\tau_{\mathrm{o}_{n}}=-u_{n} \cdot s_{n} \cdot
$$




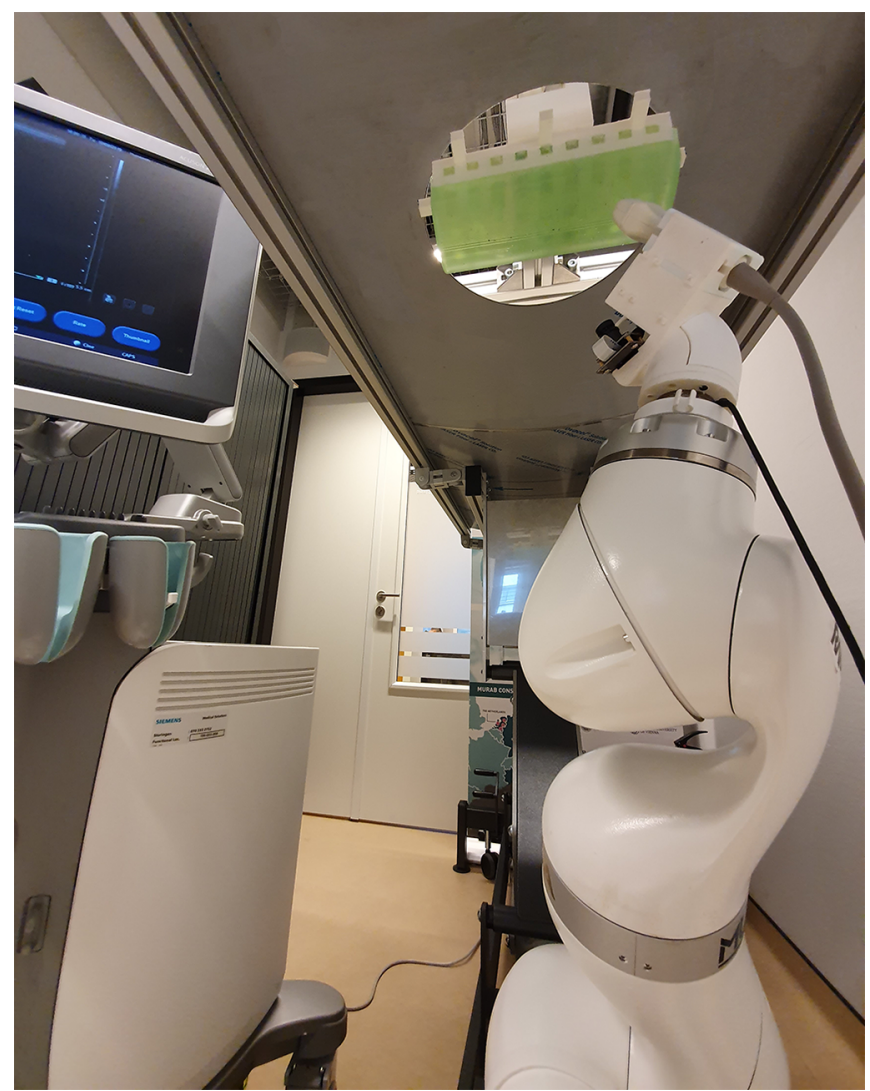

Figure 4.3: The setup used for the experiments.

\subsection{Experimental validation}

\subsubsection{Experimental setup}

An experimental setup was devised on which the designed controller was implemented and tested (Figure 4.3). It consists of a KUKA LBR Med 7 R800 (KUKA GMBH, Germany). The manipulator is equipped with an EE holding a linear probe (VF13-5, Siemens AG, Germany). The probe to flange transformation is extracted from the CAD data of the EE. The US probe is connected to a US machine (X300, Siemens AG, Germany), which streams the US images with an update rate of $24 \mathrm{~Hz}$ to a workstation via a capture card (Pro Capture DVI HD, Magewell, China). Each US image is resized to 100 by 145 pixels before converting it to a confidence map to achieve a $24 \mathrm{~Hz}$ update frequency of the visual servoing algorithm also. The workstation communicates with the 
manipulator via the fast research interface (FRI) with an update rate of $200 \mathrm{~Hz}$.

A phantom was constructed utilizing polyvinyl chloride plastisol (PVCP) (Lureparts, The Netherlands). The shape, shown in Figure 4.2, is chosen to specifically test how the system responds to both the required in-plane rotations and z-translation to keep contact.

\subsubsection{Experiments}

Four paths over the phantom were generated utilizing the phantom's CAD file. Path 1 contains homogeneous matrices as generated by the planning algorithm. In paths 2 and 3, the rotational and z-translational components were kept constant, respectively. Thus, the algorithm either compensates only for in-plane rotations or z-translations. Path 4 contains the correct $\mathrm{x}$ - and $\mathrm{y}$-coordinates, and the controller has to compensate for both in-plane rotations and z-translations.

\subsubsection{Results}

Each described path was executed five times with (w/) and without (w/o) the visual servoing controller activated, and averaging was applied. Table 4.1 shows an overview of the US acquisition results during scanning of each path w/ and w/o controller. The average mean confidence $C_{\text {mean }}$ of all images during a scan is taken as a quality measure. Three out of four paths show an improvement in mean confidence. In path 1 , a decrease in confidence of $5.9 \%$ is observed. In path 2 , the smallest improvement in confidence is achieved. The reference path contained the correct z-positions, and therefore, the probe was always half in contact with the phantom when not using the controller. Paths 3 and 4 show the most significant improvements since the lack of correct z-data meant that the probe mostly lost contact with the phantom if the controller was not activated.

Table 4.1: An overview achieved average $C_{\text {mean }}$ during the US acquisitions for each path with $(\mathrm{w} /)$ and without (w/o) the controller activated. The ratio is defined by dividing the average confidence $\mathrm{w} /$ controller by the average confidence w/o controller.

\begin{tabular}{|c|c|c|c|}
\hline Path & Controller & Avg. $C_{\text {mean }}$ & Ratio \\
\hline 1 & $\begin{array}{l}\text { w/o } \\
\text { w/ }\end{array}$ & $\begin{array}{l}0.911 \\
0.857\end{array}$ & 0.941 \\
\hline 2 & $\begin{array}{l}\mathrm{w} / \mathrm{o} \\
\mathrm{w} /\end{array}$ & $\begin{array}{l}0.768 \\
0.857\end{array}$ & 1.116 \\
\hline 3 & $\begin{array}{l}\mathrm{w} / \mathrm{o} \\
\mathrm{w} /\end{array}$ & $\begin{array}{l}0.232 \\
0.840\end{array}$ & 3.621 \\
\hline 4 & $\begin{array}{l}\mathrm{w} / \mathrm{o} \\
\mathrm{w} /\end{array}$ & $\begin{array}{l}0.203 \\
0.818\end{array}$ & 3.971 \\
\hline
\end{tabular}



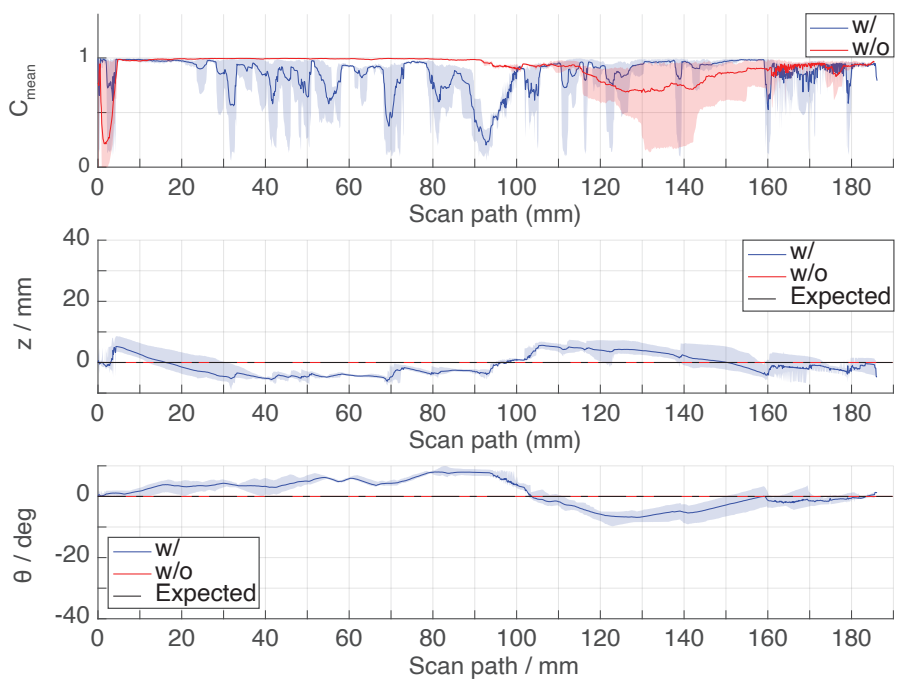

(a)
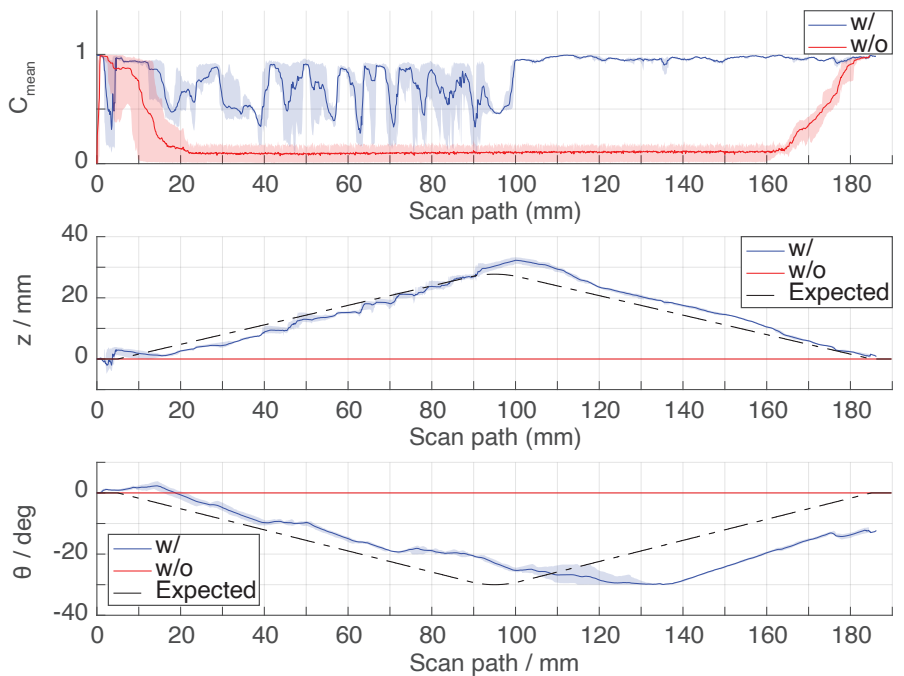

(b)

Figure 4.4: The mean confidence (top), z-adjustments (middle) and $\theta$-adjustments (bottom) for paths 1 (a) and 4 (b) plotted over the length of the traveled path for scans with (w/) and without (w/o) the controller activated. The opaque regions show minimum and maximum values measured during 5 scans. In the middle and bottom graph, the dashed line indicates the expected compensation necessary to align the probe with the phantom surface. 


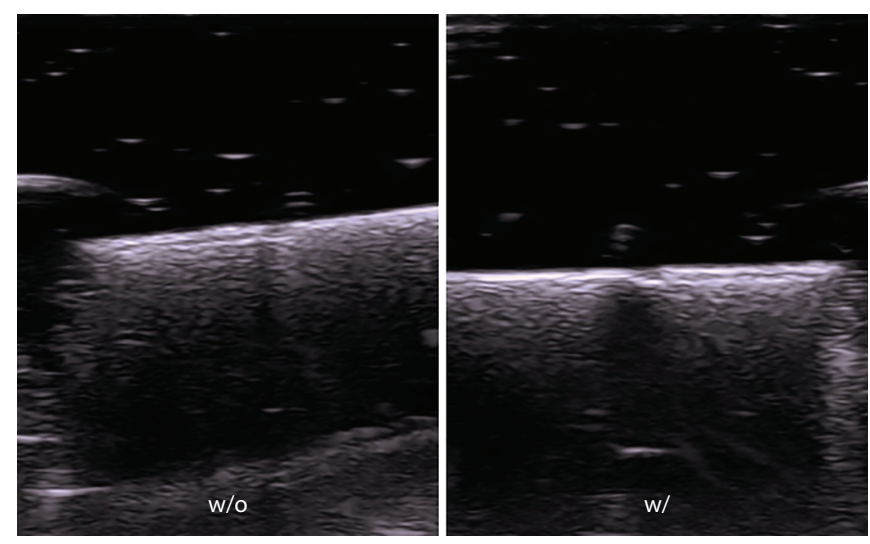

Figure 4.5: A US image of path 1 taken at $76 \mathrm{~mm}$ from the start for both w/o controller and $\mathrm{w} /$ controller. The high intensity line is the bottom of the phantom. The right image is less compressed and more balanced.

In Figure 4.4 (a) presents the measurement data of path 1. The path described the phantom surface; thus, no corrections of the visual servoing algorithm were expected. However, the $\theta$ - and z-adjustment were non-zero during most of the trajectory. Also, the trajectory w/o controller had lower confidence in the second half of the trajectory. The most probable cause is that the robot to phantom calibration - which was done by hand - was inaccurate, and thus, the phantom had a different position than the generated waypoints. Other causes could include inaccuracies in the US probe to flange calibration or the phantom surface compared to the reference path. The US images presented in Figure 4.5 show how the phantom was more compressed w/o controller than it was w/ controller at $76 \mathrm{~mm}$. Based on these results, the controller seems to compensate for biases in the phantom's position relative to the followed path.

Figure 4.4(b) presents an overview of path 4. The contact was lost shortly after the start of scanning w/o controller. This was anticipated, as the reference path only contained the correct $\mathrm{x}$ - and $\mathrm{y}$-coordinates. During scanning $\mathrm{w} /$ controller, the average mean confidence was 0.818 , which is an improvement of $397.1 \%$ compared to scanning w/o controller. Figure 4.4(b) shows that the confidence was less stable during the first half of scanning. During this half, the phantom slope moves away from the transducer. The slope moves towards the transducer during the second half, and thus, the confidence is more stable. The z- and $\theta$ - adjustments are shifted with respect to the expected adjustments, indicating that the measured phantom position deviated from its actual position. 


\subsection{Discussion}

This study demonstrates an approach to automatic robotic 3D breast US acquisition. The approach focuses on optimizing acoustic coupling, minimizing deformation and assuring patient safety. We show how a patient-specific trajectory is generated and how the trajectory is adjusted based on confidence maps. The output is passed to the SAIP controller actuating a seven-degrees-of-freedom manipulator. Although more elaborate testing is needed, the current results indicate the system can reproducibly perform US acquisitions: Table 4.1 shows that with the visual servoing algorithm activated, the average confidence values over a trajectory are pretty similar. Overall, the results show that the acquisition benefits from US feedback, either deformation-wise or image quality-wise. Our results for in-plane rotations based on confidence maps are comparable to other studies which implemented this feature 69, 73, 167, 168]. Additionally, we show that confidence maps can also be used to maintain contact with the patient. Other studies used a force sensor for maintaining probe contact. The consistency and the ability to generate patient-specific trajectories mean that the setup has an advantage over current handheld and automated methods used in clinical practice.

The setup mimicked a realistic clinical setting in which the robot is placed underneath the patient and scans the patient in prone position. Nevertheless, the setup is still experimental, and there are multiple aspects left to explore.

Firstly, we assumed that an MRI is available of the breast to generate a trajectory. MRI/US fusion can be instrumental in performing robot-assisted US-guided biopsies on lesions detected on an MRI. However, to make the system stand-alone and decrease the cost of a robotic US acquisition, a scanner like the one utilized in 163 could be added.

Further, the present phantom is not a breast phantom and is merely designed to test the algorithm's response to in-plane rotations and z-translations. A breast phantom will require a more complex trajectory but may be more suitable for the visual servoing algorithm since the changes in surface contact are less abrupt.

Currently, a PD controller is used to adjust the trajectory. The results show that the mean confidence during a scan can be more stable and differs in the second half of the trajectory compared to the first half. Though this had partly to do with the complex phantom design, better tuning or other controller implementations may improve the results.

Although a force sensor is currently not integrated into the setup, it may still be practical to do this. Firstly, gathering force data during experiments may be sensible to support our claim that less force is exerted. Additionally, a combination of US and force data can be utilized to change the desired mean confidence based on tissue deformation adaptively.

Further analysis is the SAIP controller may reveal the energy budgets and energy and power limitations appropriate for this particular application. 


\subsection{Conclusion}

We proposed an approach for robotic 3D US acquisition of the breast. The presented controller increases the average mean confidence of the ultrasound images during a trajectory in case some information of the patient-specific trajectory is missing. Also, the controller can correct for misalignments of the patient relative to the planned trajectory. Although presented for breast volume acquisitions, the approach is flexible enough for other applications. 


\section{Out-of-plane corrections for autonomous robotic breast ultrasound acquisitions}

Adapted from:

M. K. Welleweerd, A. G. de Groot, V. Groenhuis, F. J. Siepel, and S. Stramigioli, "Out-of-Plane Corrections for Autonomous Robotic Breast Ultrasound Acquisitions," in 2021 IEEE International Conference on Robotics and Automation (ICRA), 2021, pp. 12515-12521, ISBN: 9781728190778. DOI: 10.1109/ ICRA48506.2021.9560865

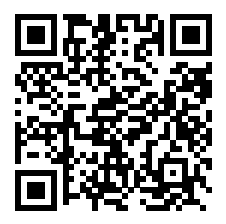




\begin{abstract}
Breast cancer affects one in eight women. Ultrasound (US) plays a vital role in the diagnostic workflow, especially during the biopsy phase, in which tissue is extracted from the lesion for further analysis. The extension from $2 \mathrm{D}$ to $3 \mathrm{D}$ US acquisitions has multiple benefits, including enhanced lesion localization and improved registration with magnetic resonance imaging data. Current commercial 3D US systems cannot preserve the breast's original shape. Robotic US scanners follow tailored trajectories and produce high-quality volumes by accurate localization of $2 \mathrm{D}$ slices captured with a conventional linear probe. Current methods require a patient-specific model to plan the scanning trajectory.

In this study, we investigate how to change the direction of the scanning trajectory based on US feedback, such that no patient-specific model is required to perform a scan. In our method, the scanning trajectory is kept tangent to the breast based on confidence maps of the US images and estimations of the current radius of curvature of the surface. We evaluated our approach on a realistic breast phantom. The robot revolves around the breast without prior knowledge of its shape. In ten scans, the root mean square error between the probe's scanning plane and the breast's surface normal is $12.6^{\circ}$ out-of-plane and $4.3^{\circ}$ in-plane. A 3D US reconstruction shows the acquired data. This is a step forward to fully autonomous, high-quality robotic US volume acquisitions.
\end{abstract}




\subsection{Introduction}

One in eight women is affected by breast cancer during her lifetime. Early detection of suspicious lesions is known to reduce the mortality rate 28. Several imaging modalities play an essential role in detecting and diagnosing breast cancer, such as mammography, ultrasound (US) and magnetic resonance imaging (MRI).

The role of US in breast cancer diagnostics is versatile. A biopsy is required if a lesion is found during an examination. This is a procedure in which some tissue from the abnormality is removed with a needle for further examination. A US-guided biopsy is the preferred biopsy method since this method gives real-time feedback, is straightforward, relatively cheap, fast and causes less patient discomfort than an MRI-guided biopsy. Additionally, US can play a role in detecting breast cancer in females with dense breasts 174.

However, US has some limitations as well. The sensitivity of US is low compared to, e.g., MRI. Therefore, lesions detected on MRI may be difficult to see on US. Additionally, B-mode US images represent $2 \mathrm{D}$ cross-sections of the tissue. These challenges, combined with the fact that the probe is manipulated manually, make interpreting the spatial relation between imaged regions complex and screening the entire breast time-consuming. Due to these limitations, a US-guided biopsy may not be possible on an MR-detected lesion, and therefore an MR-guided biopsy is necessary.

The extension of 2D US to 3D US images will partly solve these issues. A 3D US volume has multiple advantages over 2D US images: the interpretation of spatial relations between internal structures is independent of the radiologist's ability to interpret individual slices, the lesion size is measured more accurately, the reproduction of cross-sections at follow up studies is more straightforward, and the registration of US data with MRI data is less complex due to more available features 175 .

Therefore, various solutions to produce 3D US volumes have been presented. A 3D US probe can be realized by integrating a motorized 1D array of transducer elements or extending to a 2D array of elements. These probes are suboptimal since the fabrication is complex and latency of the image generation combined with the unstable hand of the radiologist introduces errors $[162$. Therefore, many systems work with regular linear probes, of which the motions are tracked through time and space. Examples of these are optically or electromagnetically tracked freehand techniques [176]. Furthermore, linear probes can be integrated on moving platforms to perform a reproducible tracked motion. Commercial breast volume scanners are available in supine and prone variations. Supine examinations cause significant deformation of the breast 163. Prone examinations, such as US tomography, cause less deformation, but the covered volume is limited, and the system is not suitable for all breast shapes and sizes [56].

Theoretically, robots are ideal for performing 3D US acquisitions because 
they produce reproducible, precisely tracked motions. This results in evenly spaced US images and eases the volume reconstruction. Multiple degrees of freedom (DOFs) allow for complex trajectories adapted to the individual's breast shape, and robots do not suffer from fatigue.

Usually, robotic US volume acquisitions consist of two steps: localization and scanning. The patient should be localized to plan the subsequent scanning phase. Current methods are surface reconstruction based on stereo cameras [68] or a depth camera 163 and the registration of MRI data based on multi modality markers 170]. Patient-specific paths may be generated by projecting a generic path on a tessellated surface representing the patient [139].

Although the patient's position was determined during the localization phase, the pre-planned path may not follow the breast's shape perfectly. This can be due to inaccuracies in the surface reconstruction or involuntary movements of the patient, such as breathing. There are several methods to compensate for inaccuracies of the pre-planned trajectory compared to the actual patient. Impedance control is often utilized to account for minor deviations and ensure safe interactions between the robot, the patient, and the radiologist $71,99,177,178$. However, some form of feedback during scanning is preferred to ensure good acoustic coupling of the US probe with the skin. Sensing mechanisms currently employed in automated scanning are force feedback and image feedback. Force feedback is mainly used to keep a constant pressure during scanning as well as to align the probe with the surface normal of the tissue $69671,73,163,179$. Kim et al. [76] link the applied force to the image quality. Our previous work showed that confidence maps are also an option to keep the US probe in contact with the tissue 139. The advantage of image feedback over a constant normal force is the application of similar deformations for both softer and harder tissue. Additionally, confidence maps have been used to balance the probe contact with the tissue $69,70,73,139$. Other visual servoing techniques, which connect end-effector (EE) behavior to image features are intensity-based methods, feature tracking algorithms, image moments and speckle correlation or block matching 75, $77,79,169$.

In this paper, we investigate how to utilize current and past US images to perform corrections on the path not only in-plane, like in our previous work [139], but also out of the US plane. The advantage is that the robot will find its way around the breast autonomously, and thus the localization step can be omitted. The out-of-plane corrections are achieved by keeping the confidence constant and estimating the radius of curvature. The probe's scanning plane is kept perpendicular to the tangent plane of the surface and thus follows the breast's shape. The approach is validated utilizing experiments on a realistic breast phantom. 


\subsection{The scanning algorithm}

\subsubsection{System overview}

Figure 5.1 shows an overview of the system. The patient lies in prone position on a bed with the examined breast through a hole such that it is freely accessible by the robot. The robot is placed underneath the bed and is equipped with an EE carrying a US probe [170]. The robot's initial position coincides with the first pose and position of the initially planned trajectory. This trajectory is a straight line at a specified height in the negative y-direction of the EE frame, $\Psi_{\mathrm{ee}}$ Once the scanning is started, the robot first localizes the breast surface. During scanning, the robot tries to keep the trajectory tangent to the surface based on the confidence of incoming US images (Figure 5.1(b)). It does so by transforming the remaining part of the trajectory with the depicted transformations. The functioning of the various components is further elaborated in the following sections.

\subsubsection{Operational space control}

Operational space control, originally introduced by [180], is an approach to achieve desired EE behavior by applying virtual forces to the EE and mapping these to the joint space of the robot. The control signal for the torques on the joints, $\boldsymbol{\tau}_{\mathrm{c}}$, is expressed as

$$
\boldsymbol{\tau}_{\mathrm{c}}=\boldsymbol{J}^{\top}(\boldsymbol{q}) \boldsymbol{M}_{x}(\boldsymbol{q})\left(\boldsymbol{k}_{\mathrm{p}}\left(\boldsymbol{x}_{\mathrm{d}}-\boldsymbol{x}\right)+\boldsymbol{k}_{\mathrm{d}}\left(\dot{\boldsymbol{x}}_{\mathrm{d}}-\dot{\boldsymbol{x}}\right)\right)
$$

where $\boldsymbol{q}$ is the vector with joint positions, $\boldsymbol{J}^{\top}(\boldsymbol{q})$ is the Jacobian transpose, which maps the forces from the operational space to the joint space, $\boldsymbol{M}_{x}$ is the inertia matrix of the robot expressed in the operational space, which is

$$
\boldsymbol{M}_{x}(\boldsymbol{q})=\left(\boldsymbol{J}(\boldsymbol{q}) \boldsymbol{M}_{\mathrm{q}}^{-1}(\boldsymbol{q}) \boldsymbol{J}^{\top}(\boldsymbol{q})\right)^{-1},
$$

in which $\boldsymbol{M}_{\mathrm{q}}(\boldsymbol{q})$ is the mass matrix of the robot expressed in joint space. $\boldsymbol{k}_{\mathrm{p}}$ is the spring constant, $\boldsymbol{x}_{\mathrm{d}}$ and $\dot{\boldsymbol{x}}_{\mathrm{d}}$ are the desired position and velocity, respectively, $\boldsymbol{x}$ and $\dot{\boldsymbol{x}}$ are the current position and velocity in the operational space, respectively, and $\boldsymbol{k}_{\mathrm{d}}$ is the value of the damper. $\boldsymbol{x}_{\mathrm{d}}$ is extracted from a homogeneous transformation matrix, $\boldsymbol{H}_{\mathrm{d}}^{0}$, which is generated by the visual servoing algorithm and describes the desired configuration of the EE-frame, $\Psi_{\text {ee }}$, with respect to the base frame, $\Psi_{0}$. In our work, the desired velocity, $\dot{\boldsymbol{x}}_{\mathrm{d}}$, is zero.

\subsubsection{Confidence maps}

Karamalis et al. [172] originally introduced confidence maps to highlight attenuated regions of a US image. As such, they are useful to gain insight into the 


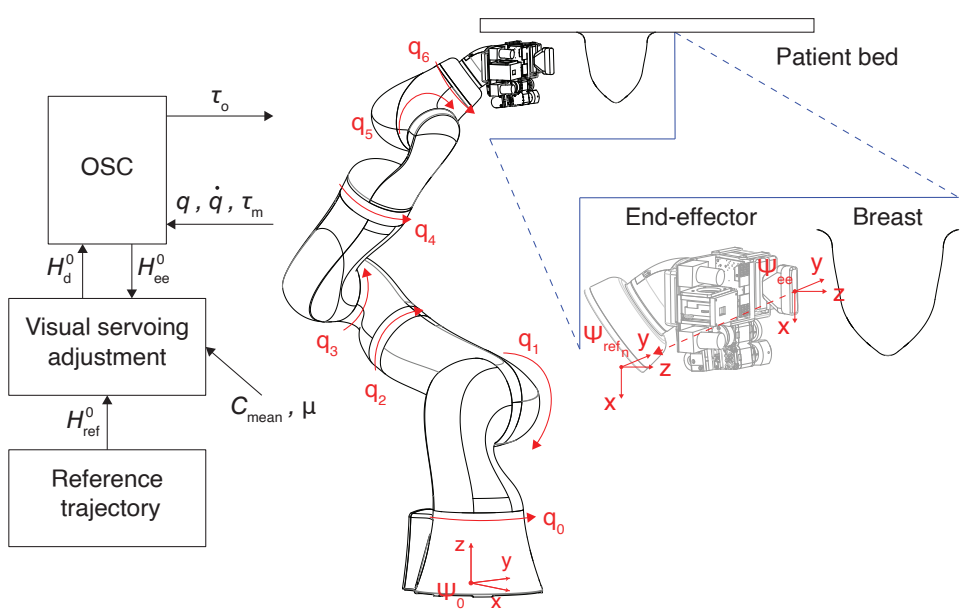

(a)
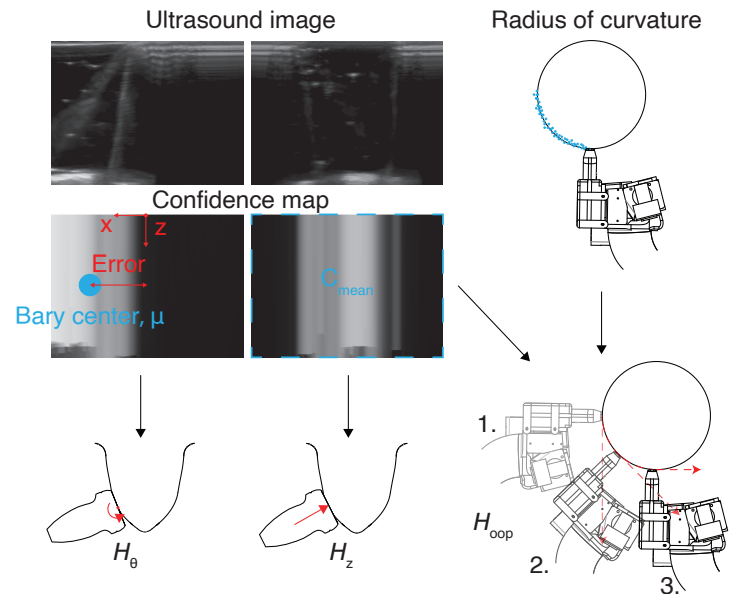

(b)

Figure 5.1: A system overview. (a) A 7-DOFs manipulator with an end-effector is placed underneath the patient's breast. The reference trajectory is initially a straight line, but is wrapped around the breast in real-time based on the visual servoing algorithm. (b) Three probe adjustments can be performed based on US feedback and past EE positions: a rotation around the EE $\mathrm{y}$-axis based on the bary center, a movement in the z-direction based on the mean confidence, or a rotation around the $\mathrm{x}$-axis based on the current radius of curvature and the mean confidence. 
acoustic coupling of the probe with the skin. Each pixel $(u, v)$ of an image is located in $\Omega:=[1 . . n] \times[1 . . m]$. A confidence map $C: \Omega \rightarrow[0,1]$ is a pixel-wise representation of the uncertainties in a US image $U: \Omega \rightarrow[0,1]$. The map, $f: U \rightarrow C$, is based on the probability of a random walk starting from a pixel and reaching each virtual transducer element. The random walk equilibrium meets three constraints: the top row of the US image has confidence 1, the bottom row has confidence 0 , and the signal path conforms to US-specific propagation constraints. In Figure 5.1(b), examples of US images and their corresponding confidence maps are presented. Two features of the confidence maps are useful for adjustments of the pose and position of the probe: the mean confidence, $C_{\text {mean }}$, and the barycenter of the confidence, $\mu$.

The mean confidence is a measure for the area of the transducer being in contact with the skin. It is defined as

$$
C_{\text {mean }}=\frac{1}{n \cdot m} \sum_{(u, v) \in \Omega} C(u, v) .
$$

If the mean confidence is controlled around a setpoint, $C_{\mathrm{s}}$, a constant amount of contact with the skin is ensured. The confidence error is defined as: $e_{\mathrm{C}}=$ $C_{\mathrm{s}}-C_{\text {mean }}$.

The barycenter of the confidence is defined as

$$
\begin{aligned}
\mu_{u} & =\frac{1}{C_{\Omega}} \sum_{(u, v) \in \Omega} u \cdot C(u, v), \\
\mu_{v} & =\frac{1}{C_{\Omega}} \sum_{(u, v) \in \Omega} v \cdot C(u, v),
\end{aligned}
$$

with $C_{\Omega}=\sum_{(u, v) \in \Omega} C(u, v)$ the total confidence. The pixel indices $\mu_{u}$ and $\mu_{v}$ correspond to EE coordinates $\mu_{z}$ and $\mu_{x}$, respectively. A centered contact will result in $\mu_{x}=0$. As in-plane rotations have an influence on the barycenter, the error is defined as $e_{\mu}=\arctan \frac{\mu_{x}}{\mu_{z}}$ radians.

The controller only utilizes the top of the confidence image to make both features independent of the patient's physiology; the first layer of tissue always consists of skin and fatty tissue.

\subsubsection{Continuous contact}

A controller is designed to maintain the mean confidence and the barycenter at the setpoints and, as a result, keep contact during a scan. Initially, the planned path is a straight line; its waypoints are listed in an array of transformation matrices, $\boldsymbol{H}_{\mathrm{ref}}^{0}(j), j$ indicating the current entry.

As outlined in Figure 5.1(b), three types of transformations are applied to the initial trajectory: $\boldsymbol{H}_{\theta}$ rotates the probe around its y-axis, $\boldsymbol{H}_{z}$ is adjusted to 


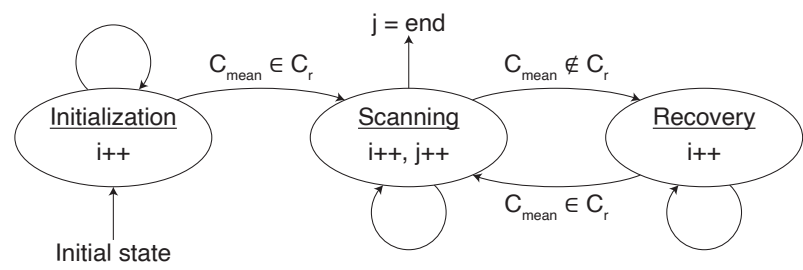

Figure 5.2: The robot controller has three states: The initialization state, the scanning state and the recovery state. The initialization state ensures the initial contact. The scanning state takes new waypoints and continues the trajectory along the breast. The recovery state regains contact if the confidence is insufficient.

translate the waypoints towards or away from the breast, and the out-of-plane transformation, $\boldsymbol{H}_{\text {oop }}$, is used to give the trajectory a new direction. The desired configuration of the EE in the $i$-th control iteration, $\boldsymbol{H}_{\mathrm{d}}^{0}(i)$, is defined as

$$
\boldsymbol{H}_{\mathrm{d}}^{0}(i)=\boldsymbol{H}_{\mathrm{oop}}(i) \boldsymbol{H}_{\mathrm{ref}}^{0}(j) \boldsymbol{H}_{z}(i) \boldsymbol{H}_{\theta}(i) .
$$

$\boldsymbol{H}_{\theta}(i)$ is adjusted to maintain the confidence barycenter at its setpoint. This is performed by a PID controller, and the updated matrix is defined as:

$$
\boldsymbol{H}_{\theta}(i)=\boldsymbol{H}_{\theta}(i-1)\left[\begin{array}{cc}
\operatorname{Rot}_{y}(\theta) & \mathbf{0}^{3 \times 1} \\
\mathbf{0}^{1 \times 3} & 1
\end{array}\right],
$$

in which $i-1$ indicates the previous control iteration and $\operatorname{Rot}_{y}(\theta)$ is a rotation matrix around the y-axis of $\theta$ radians.

A state machine is designed to apply modifications to $\boldsymbol{H}_{z}(i)$ and $\boldsymbol{H}_{\text {oop }}(i)$ in a coordinated manner (Figure 5.2. It consists of three states: initialization, scanning and recovery.

\section{Initialization state}

This state ensures acoustic coupling between the breast surface and the US probe at the start of the scan. To achieve this, next to $\boldsymbol{H}_{\theta}(i), \boldsymbol{H}_{z}(i)$ is manipulated by a PID controller. $\boldsymbol{H}_{z}$ is defined by

$$
\boldsymbol{H}_{z}(i)=\boldsymbol{H}_{z}(i-1)\left[\begin{array}{cc}
\boldsymbol{I}^{3 \times 3} & d \hat{\boldsymbol{z}} \\
\mathbf{0}^{1 \times 3} & 1
\end{array}\right],
$$

in which $\boldsymbol{I}$ is the identity matrix, $d$ is the displacement and $\hat{\boldsymbol{z}}$ is the unity vector in the z-direction.

A mean confidence range, $C_{\mathrm{r}}$, has been defined with the lower and upper boundary being $b_{\min }$ and $b_{\max }$, respectively, such that: $C_{\mathrm{r}}=\left[b_{\min }, b_{\max }\right]$. The probe is in contact if $C_{\text {mean }} \in C_{\mathrm{r}}$ and the controller moves on to the next state. In this state: $\boldsymbol{H}_{\mathrm{oop}}=\boldsymbol{I}^{4 \times 4}$. 


\section{Scanning state}

The robot moves along the path by incrementing $j$ every control iteration. $\boldsymbol{H}_{\text {oop }}(i)$ is adjusted to maintain the confidence setpoint. If the confidence decreases while performing a linear motion, the surface is expected to be convex, whereas increasing confidence indicates a concave surface. As such, a PID controller can adjust the direction of the scan to maintain the confidence setpoint. Additionally, the radius of curvature of the surface is estimated by applying a Taubin circle fit on a window of the past trajectory 181 . Therefore, a prediction of the change in direction is available as well.

The out-of-plane transformations perform a rotation around a frame defined by the orientation of the reference path and the origin of the current EE position. The transformation is expressed in the base frame of the robot such that $i$-th out-of-plane rotation is defined as:

$$
\begin{aligned}
& \boldsymbol{H}_{\mathrm{oop}}(i)=\boldsymbol{H}_{\mathrm{oop}}(i-1) \cdot \\
& {\left[\begin{array}{cc}
\boldsymbol{R}_{\mathrm{ref}}^{0} & \boldsymbol{p}_{\mathrm{ee}}^{0} \\
\mathbf{0}^{1 \times 3} & 1
\end{array}\right]\left[\begin{array}{cc}
\operatorname{Rot}_{x}(\phi)^{3 \times 3} & \mathbf{0}^{3 \times 1} \\
\mathbf{0}^{1 \times 3} & 1
\end{array}\right]\left[\begin{array}{cc}
\boldsymbol{R}_{\mathrm{ref}}^{0} & \boldsymbol{p}_{\mathrm{ee}}^{0} \\
\mathbf{0}^{1 \times 3} & 1
\end{array}\right]^{-1},}
\end{aligned}
$$

in which $\boldsymbol{R}_{\mathrm{ref}}^{0}$ is the orientation of the current reference waypoint, $\boldsymbol{p}_{\mathrm{ee}}^{0}$ is the current position of the EE and $\operatorname{Rot}_{x}(\phi)$ is a rotation around the x-axis by $\phi$ radians. In this state, $\boldsymbol{H}_{z}$ is kept constant. The controller goes to the recovery state if $C_{\text {mean }} \notin C_{\mathrm{r}}$.

\section{Recovery state}

The controller adjusts $\boldsymbol{H}_{z}(i)$ to regain sufficient contact with the skin. Additionally, $\boldsymbol{H}_{\mathrm{oop}}(i)$ is adjusted such that the EE is again perpendicular to the newly estimated tangent. The controller moves back to the scanning state if $C_{\text {mean }} \in C_{\mathrm{r}}$.

Table 5.1 gives an overview of which manipulation is performed in which state of the state machine. 


\subsection{Experimental validation}

\subsubsection{Experimental setup}

The setup (Figure 5.3 consists of a 7-DOFs robotic manipulator (KUKA Med 7 R800, KUKA GmbH, Germany) to which an EE is connected. The EE holds an L12-5L40S-3 linear US probe (Telemed UAB, Lithuania). The transformation of the transducer with respect to the flange is retrieved from the computeraided design file of the EE. The US probe is connected to a MicrUs EXT-1H (Telemed UAB, Lithuania), which streams the $27 \times 40 \mathrm{~mm}(\mathrm{w} \times \mathrm{h})$ US images with an update rate of $40 \mathrm{~Hz}$ to a workstation via a server. This workstation runs the algorithms and communicates via the fast research interface with the manipulator 159 .

A breast phantom was made based on the surface reconstruction of a breast MRI of a woman lying in prone position. Two molds were printed: an outer mold with the breast shape and an inner mold. The inner mold was placed inside the outer mold to create a $5 \mathrm{~mm}$ thick skin layer, which was filled with a polyvinyl chloride plastisol (PVCP)/Plasticizer (100\% / $0 \%$ ) mixture (Bricoleurre, France). Then, the inner mold was removed. The remaining volume was filled with a mix of PVCP/Plasticizer (100\%/0\%) strands and PVCP/Plasticizer $(70 \% / 30 \%)$ to create a randomized structure resembling the fatty and glandular tissue in an actual breast. Silica powder was added to all mixtures in varying amounts

Table 5.1: Overview of the various manipulations to the current desired position and the corresponding states in which they are performed.

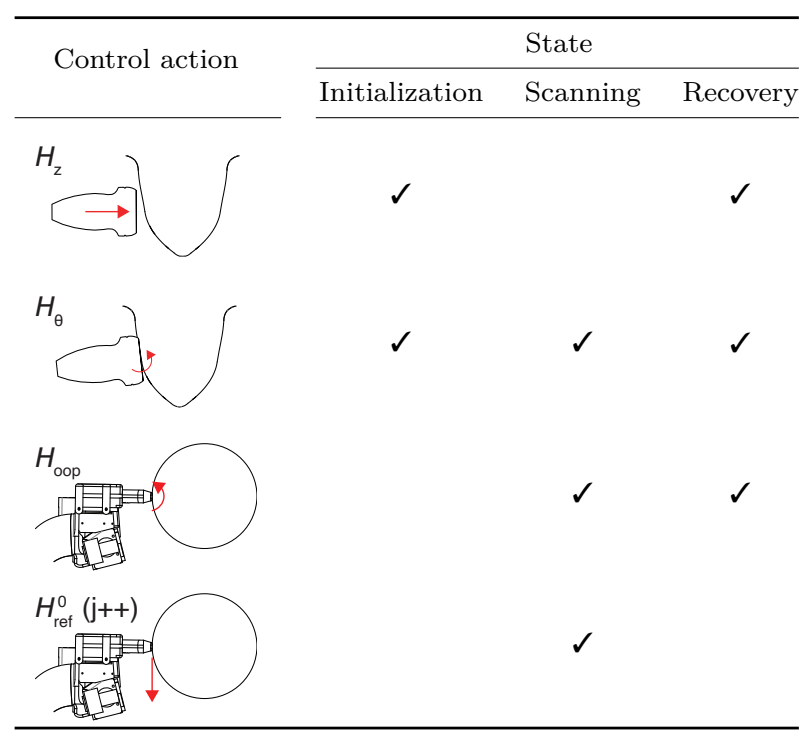




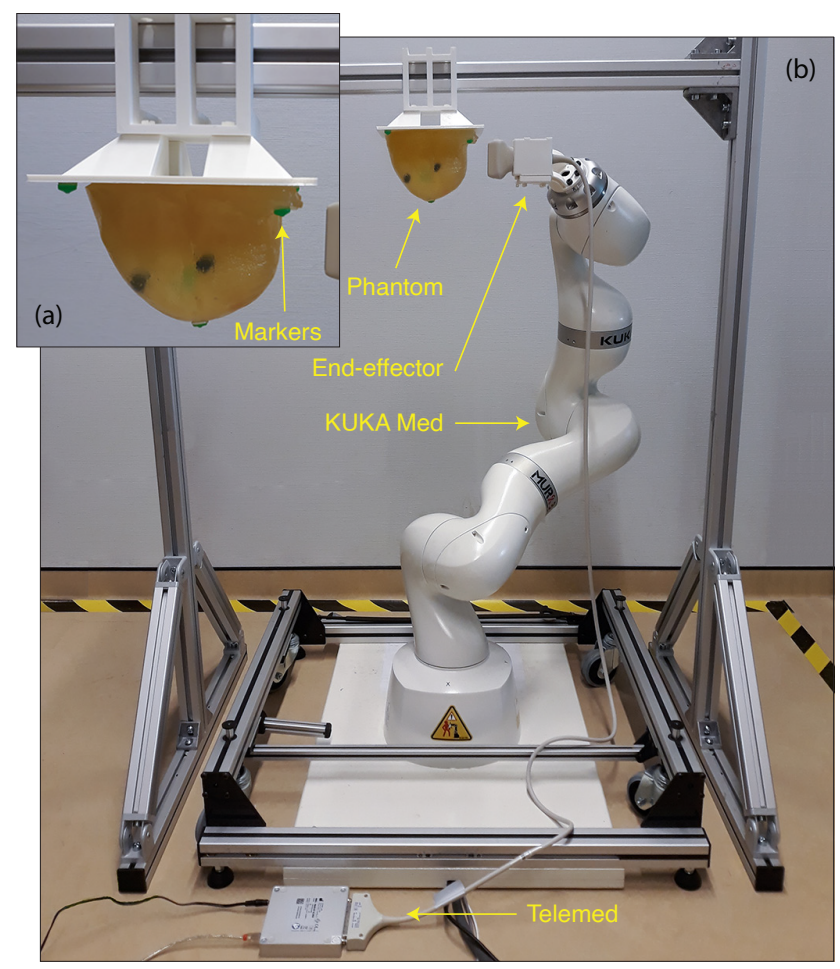

Figure 5.3: The setup. (a) A close-up of the EE and the phantom with the skin markers indicated. (b) An overview of the setup with indicated: the KUKA MED medical robot, the $\mathrm{EE}$, the phantom and the Telemed ultrasound machine.

$(0-1 \mathrm{wt} . \%)$ to give the tissue varying degrees of echogenicity. The fatty tissue in this breast is up to ten times stiffer than actual fatty tissue; the skin layer has a stiffness comparable to real skin 182 184.

Five skin markers were fabricated from PVCP mixed with a green colorant (LUPA coloring, LureParts, The Netherlands). These were attached to a $1 \mathrm{~mm}$ PET disk and glued on the phantom. The markers are used for MRI data to robot registration during the experiment.

The breast was placed centered above the robot at a height of approximately $1.1 \mathrm{~m}$ (Figure 5.3). This configuration resembles a patient lying in prone position on the bed.

\subsubsection{Experiments}

The fabricated phantom was scanned ten times. A scan contained the following steps: US gel was applied to the phantom with a brush. The EE was aligned with 


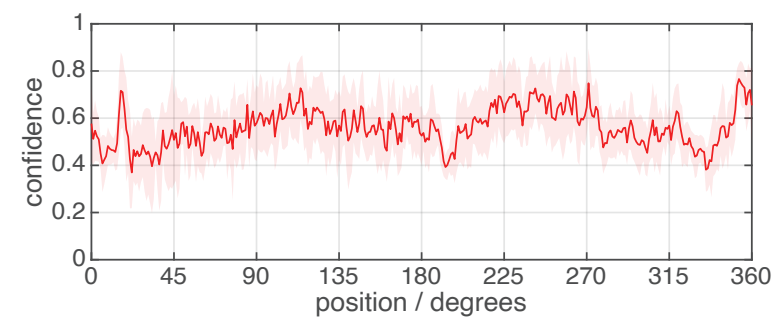

(a)

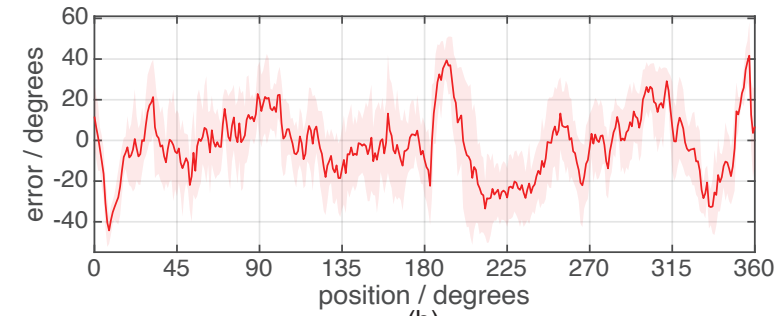

(b)

Figure 5.4: (a) The average confidence, $C_{\text {mean }}$ along the trajectory for ten scans. (b) The average error of the barycenter, $e_{\mu}$, for ten scans. The opaque region indicates the standard deviation.

the first location of the path, $(x, y, z)=(0.06,0,1.01) \mathrm{m}$. The $\mathrm{y}$ - and z-axis of the EE were aligned with the $-\mathrm{y}$ and $\mathrm{x}$-axis of the base frame, respectively. The probe surface was located at $2 \mathrm{~cm}$ from the breast surface in this position. The scan was started and the robot automatically navigated along the breast surface with a velocity of $2.5 \mathrm{~mm} \mathrm{~s}^{-1}$. A scan was stopped when the robot had executed approximately $360^{\circ}$ around the breast. The confidence setpoint, $C_{\mathrm{s}}$, was 0.50 . The confidence range in which the scanning state is active was $C_{\mathrm{r}}=[0.35,0.7]$. The boundaries were chosen asymmetric because higher confidence is preferred over no contact. Additionally, a phantom-to-robot calibration was performed with stereo cameras to evaluate the quality of the US acquisitions afterward.

\subsubsection{Results}

The robot successfully revolved around the breast ten times. All plots are shown in polar coordinates with the breast at its center because the trajectory approximates a circle.

In Figure 5.4 the mean confidence and the error of the barycenter are plotted. The root mean square (RMS) error of the confidence was 0.08 . The confidence is mostly above the setpoint of 0.5 because too low confidence was penalized more by the controller than too high confidence due to the asymmetric boundaries that determine the robot state. The barycenter was on average $-2.2^{\circ}$ off, whereas the RMS error is $12.2^{\circ}$. 


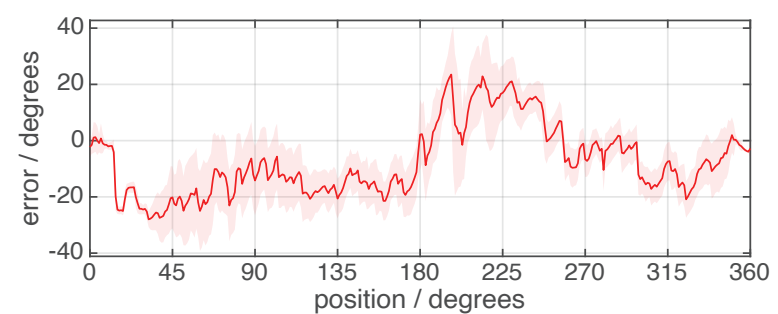

(a)

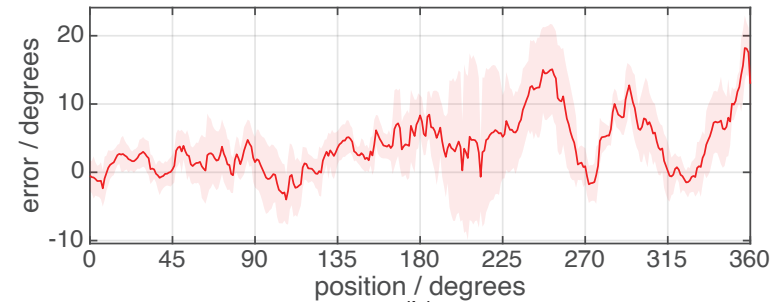

(b)

Figure 5.5: (a) The average out-of-plane error of the probe with respect to the surface normal of the breast for ten scans. (b) The average in-plane error of the probe relative to the surface normal of the breast for ten scans. The opaque region indicates the standard deviation.

The EE's z-axis should be collinear with the breast's surface normal. To this end, the probe can rotate around the EE's x- and y-axis. We assessed the EE's orientation relative to the phantom using the camera calibration and the surface reconstruction of the phantom. The out-of-plane error is defined as the angle between the breast's surface normal and the image plane. Thus, the rotation around the EE's x-axis that is necessary to align the image plane with the surface normal. The in-plane error indicates how much the rotation around the EE's y-axis is off. Fig. 5(a) and (b) present the out-of-plane and the in-plane error, respectively. On average, the out-of-plane error is $-7.6^{\circ}$, and the RMS error $12.6^{\circ}$. At $0^{\circ}$, the error builds up in the negative direction because the robot did not record enough data to estimate the radius of curvature yet. Around $180^{\circ}$, the error becomes positive since the breast's cross-section is ellipse-shaped (see Figure 5.6(a)), and the algorithm underestimates the radius of curvature on the flatter side. The mean in-plane error is $4.0^{\circ}$, and the RMS error is $4.3^{\circ}$. As expected, these numbers have the same order of magnitude as the errors in the barycenter.

Figure 5.6(a) shows the robot trajectory together with the breast surface. Based on this data, the mean deformation is $(5.3 \pm 3.5) \mathrm{mm}$, and the breast is most deformed around $0^{\circ}$ and $210^{\circ}$. This effect is also due to the algorithm's tendency to underestimate after a transition from a small to a large radius of curvature. Additionally, at $210^{\circ}$, the EE's xy-plane is almost aligned with the world's xy-plane. The steep inclination of the breast's surface at this position 
makes this plot sensitive to this section's calibration errors.

Figure 5.6. (b) shows the confidence in the US images at $z=1.01 \mathrm{~m}$ for scan No. 9. The graph shows a confidence that is higher than the average confidence as shown in Figure 5.4 (a) in most sections. The confidence is usually the highest in the middle of an image. This cross-section is taken at the middle of all acquired images and represents the highest confidence found for each location. From this image, it is clear that the breast volume is mostly covered with high confidence data. The confidence is lower in the middle because the confidence decreases the further it travels through the phantom. A section in the middle has no image data, and thus, no confidence data.

Figure 5.6(c) shows the reconstructed US cross-section. This reconstruction is based on the acquired US data, and a deformation compensation is applied based on the original shape as extracted from the MRI images of the phantom. Some features of the phantom are clearly distinguished, such as the $5 \mathrm{~mm}$ thick skin layer.

\subsection{Discussion}

This study presents an approach for fully automated robotic breast volume US acquisitions. The approach was tested in a realistic clinical setting with a breast phantom placed in prone position over the robot. The robot follows the breast surface without prior knowledge.

Our results show that it is feasible to control three DOFs of the EE based on confidence maps: a translation in the z-direction of the $\mathrm{EE}$, an in-plane rotation and an out-of-plane rotation. This is an improvement over our previous study [139], which was able to control two degrees of freedom.

The addition of an extra DOF has a significant impact on the robotic US acquisition workflow. Other studies use some form of prerequisite information on which the US scan is based. This information is often a surface reconstruction of the scanned area and can be acquired with stereo cameras [68], a depth camera [163], or registration with a MRI images [139. In our implementation, the robot revolves around the breast based on a generic initial path and US feedback. Calibration steps between the camera, the robot and the patient, the extra time taken by recording the surface data or the necessity of a preoperative MRI are all ruled out because the system depends only on US data. Additionally, the complexity of the EE is reduced to just a linear US probe.

However, the US volume reconstruction becomes more complex if the preprocedural surface reconstruction is absent because the initial state is unknown compared to the compressed state. In our study, we utilized the camera calibration and the surface reconstruction of the phantom for deformation compensation in the US volume reconstruction. Currently, we are investigating whether transitions from low to high confidence allow us to reconstruct the original breast shape 


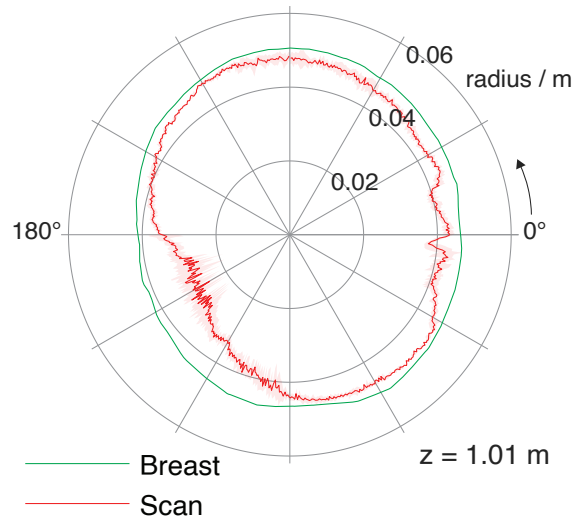

(a)

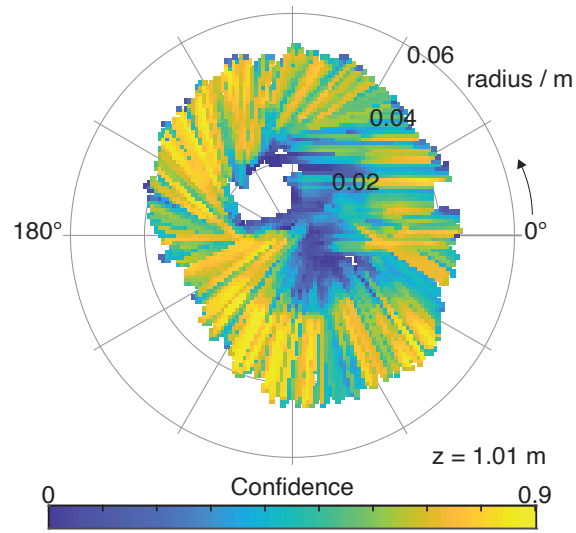

(b)

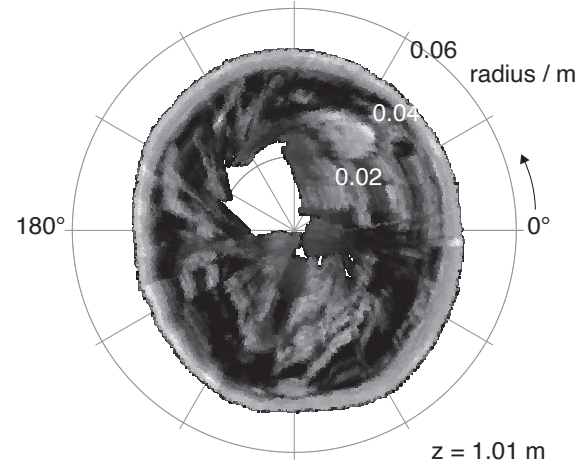

(c)

Figure 5.6: (a) The anticipated cross-section (green) of the breast at height $z=1.01 \mathrm{~m}$ and the the average US probe's position (red) for ten scans. The opaque region indicates the standard deviation. (b) The confidence in the US signal in the cross-section of the breast at $z=1.01 \mathrm{~m}$ for scan No. 9. This scan was the closest to the average trajectory shown in (a). (c) The generated US cross-section of the breast based on the acquired data during scan No. 9.

during a scan. This allows both the robotic acquisition and the reconstruction to be independent of prerequisite data.

The current implementation's out-of-plane corrections still lag behind the actual surface normal of the breast. Consequently, Figure 5.6(c) shows a section without data in the middle. The lag is caused by the system's dependence on predicting the radius of curvature, which is based on a past section of the trajectory. Therefore, the prediction is not accurate at the start of the scan. Also, later during the scan, if the radius of curvature transitions from small to large or vice versa too fast, the system adapts with a delay. Position-wise, this delay is less present because the recovery state constantly reestablishes the 
probe's contact with the skin.

The system's performance should be further examined under different circumstances. Currently, the system was tested on one phantom with a specific size, shape and material. Although the phantom is a representative example, many variations occur in real-life scenarios, such as the quantity of US gel, actual human tissue and patient movements. The robustness may be improved by manually choosing the initial position of the probe, such that the initial configuration can be adapted to the breast's shape by, e.g., an initial in-plane rotation. Differences in stiffness are expected to have a minor impact on the results since the adjustments are based on image features, not on force.

Currently, the acquisition time is approximately $180 \mathrm{~s}$, which is comparable to existing systems [56]. However, total volume coverage may require multiple sweeps at varying heights. Therefore, the scanning velocity and acquisition rate may have to be increased in future work.

Overall, the presented method shows potential for autonomous breast volume US acquisitions. The approach may also be applicable in other clinical settings, such as skeletal muscle volume determination and abdomen screening.

\subsection{Conclusion}

This work investigates how to control three DOFs of the robot utilizing US feedback. Based on a simple reference trajectory and real-time US and position feedback, the robot finds its way around a complex shape like the breast. Currently, the RMS error of the in-plane and the out-of-plane alignment of the scanning plane with the surface normal is $4.3^{\circ}$ and $12.6^{\circ}$, respectively. The deformation caused by the probe contact with the skin is on average $(5.3 \pm 3.5) \mathrm{mm}$. The acquired US data covers a significant part of the desired cross-section. The obtained results show the approach's potential, which may also be interesting for other US scanning applications. 


\section{Robot-assisted ultrasound-guided biopsy on MR-detected breast lesions}

Adapted from:

M. K. Welleweerd, D. Pantelis, A. G. de Groot, F. J. Siepel, and S. Stramigioli, "Robot-assisted ultrasound-guided biopsy on MR-detected breast lesions," in 2020 IEEE/RSJ International Conference on Intelligent Robots and Systems (IROS), IEEE, Oct. 2020, pp. 2965-2971, ISBN: 978-1-7281-6212-6. DOI: 10 1109/IROS45743.2020.9341695

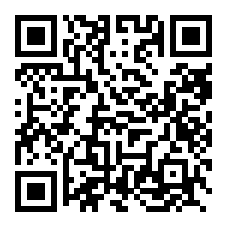




\begin{abstract}
One in eight women will get breast cancer during their lifetime. A tissue sample should be acquired from the lesion with a needle to confirm the diagnosis. The so-called biopsy is preferably executed under ultrasound (US) guidance because it is simple, fast, and cheap, gives real-time image feedback and causes little patient discomfort. However, magnetic resonance (MR)-detected lesions may be barely or not visible on US and difficult to find due to deformations of the breast. This paper presents a robotic setup and workflow that assists the radiologist in targeting MR-detected breast lesions under US guidance, taking into account deformations and giving the radiologist robotic accuracy. The setup consists of a seven degrees-of-freedom robotic serial manipulator with an end-effector carrying a US transducer and a three degrees-of-freedom actuated needle guide. During the probe positioning phase, the US probe is positioned on the patient's skin while the system tracks skin contact and tissue deformation. During the intervention phase, the radiologist inserts the needle through the actuated guide. The tissue deformation is tracked during insertion, and the needle path is adjusted accordingly. The workflow is demonstrated on a breast phantom. Experiments show that lesions with a radius down to $2.9 \mathrm{~mm}$ can be targeted. While magnetic resonance imaging is becoming more important in breast cancer detection, the presented robot-assisted approach helps the radiologist confirm the diagnosis effectively and accurately utilizing the preferred US-guided method.
\end{abstract}




\subsection{Introduction}

Breast cancer is one of the most common cancers and the leading cause of cancer death in females [28]. Successful treatment is more likely if the disease is detected and diagnosed in an early stage. While mammography is the most widespread imaging modality for detection, magnetic resonance imaging (MRI) is getting more important. MRI has a higher sensitivity than other imaging modalities and can overcome the shortcomings of mammography by using new imaging approaches. However, the selectivity of MRI, which is the ability to differentiate between benign and malignant lesions on the acquired images, is not very high. Consequently, a tissue sample from the detected lesion should be obtained to confirm the diagnosis. The preferred procedure to achieve this is the ultrasound (US)-guided biopsy. The radiologist performs this procedure, who manually inserts the biopsy needle and navigates to the lesion under US guidance. US-guided biopsies are preferred since - compared to an MRI-guided biopsy - they are relatively cheap, simple, fast, give real-time feedback and cause little patient discomfort because of the smaller needle diameters used [44].

However, performing a US-guided biopsy on an MR-detected lesion is challenging. Firstly, transferring the lesion position from the MRI to the US image is complicated due to the different patient positioning between imaging modalities; an MRI is typically performed with the patient in prone position, while during a US-guided biopsy, the patient is positioned semi-supine. Due to the highly deformable tissue, relating the US images to the MRI is difficult. Furthermore, an MR-detected lesion may be barely visible on the US image [44. Finally, the procedure is highly operator dependent due to these challenges and therefore finding the lesion may take a significant amount of time.

The field of robotics is progressively becoming more important in healthcare due to its high accuracy, efficiency and operator independence. Specifically, there is an increasing interest in robot-assisted breast biopsies because a robot can accurately position the US probe and manipulate the needle based on the target location 95 . The biggest challenges in targeting a breast lesion include initial localization of the lesion and deformations of the breast during the procedure.

Firstly, the challenge of initial localization is significantly reduced by performing both the MR-imaging and robotic biopsy with the patient prone position. Not only will involuntary movements such as breathing be less apparent in prone position, but it also allows to image the breast in its undeformed state 102]. Additionally, it is easier to relate the breast to the MRI using, e.g., markers attached to the skin 68.

Secondly, there are two approaches to correct for deformations occurring during the robot-assisted US-guided biopsy procedure: deformation prediction and deformation tracking. Prediction methods are model-based and update the lesion position based on estimations of the tissue-probe and tissue-needle interactions. Subsequently, the planned needle trajectory is optimized for this. 
Although research on the prediction of deformations resulting from different positioning of the breast or interactions with the breast is ongoing [107, 185, a sufficiently accurate patient-specific prediction model of the interactions of the US probe and the biopsy needle with the breast is currently infeasible. Therefore, robotic solutions depend mainly on deformation tracking. Current robotic breast biopsy systems mostly depend on the visibility of the target 14. 97, 98, 114, 128 and therefore, any deformations related to probe placement and needle insertion are easily compensated for in these applications; the lesion can be segmented from subsequent US images and the needle trajectory can be adjusted accordingly. Actually, a target can be tracked independently of its visibility by using tissue deformation tracking algorithms. Applicable algorithms that have been implemented in robotic US applications include speckle tracking, optical flow and normalized cross correlation and mutual information similarity functions $79,110,186]$. However, those studies do not focus on the whole breast biopsy procedure, i.e., they do not include probe positioning and the resulting deformations.

The purpose of this work is to develop a workflow to perform a robot-assisted US-guided biopsy on MR-detected lesions accurately. The work focuses explicitly on using US feedback from the probe in the process. US feedback is used to acquire acoustic coupling, which is the transfer of acoustic energy from the probe into the tissue. Most other studies use a normal force for this. Also, in the absence of accurate deformation prediction models and the ability to detect the lesion on US images, US feedback is used to compensate for deformations caused by the needle insertion and deformations caused upon probe contact. The lesion is not visible, but its initial position before deformation is retrieved from preoperative data, such as MRI.

The presented solution utilizes a seven-degrees-of-freedom (7-DOFs) serial manipulator equipped with a linear US probe and an actuated needle guide. Confidence maps, previously used for pose adjustments of robotic US systems 69 166, are used to estimate the first probe contact and place the probe correctly. Upon first probe contact, it is assumed that the deformation is not significant. The target, whose position was extracted from preoperative data, can thus be mapped in the US image and tracked with the optical flow during further positioning and needle insertion. The radiologist is responsible for needle insertion, but the actuated needle guide determines the needle trajectory based on kinematics and needle detection. The robot-assisted biopsy workflow is validated with phantom experiments. Experiments show that preoperatively defined targets that are invisible on US images are targeted with millimeter accuracy. The compensation for movements in the 2D US frame is the first step towards compensation for both in- and out-of-plane deformations. 


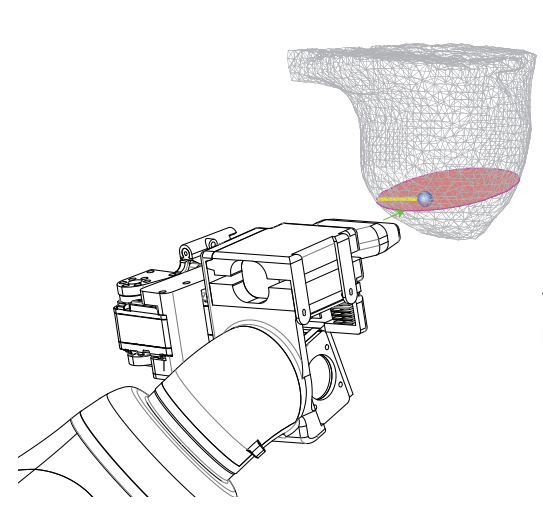

(a)

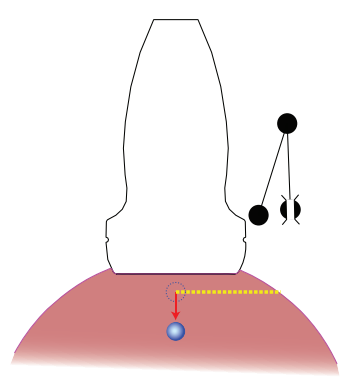

(c)

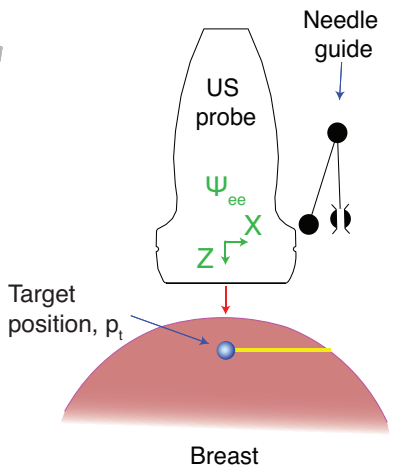

(b)

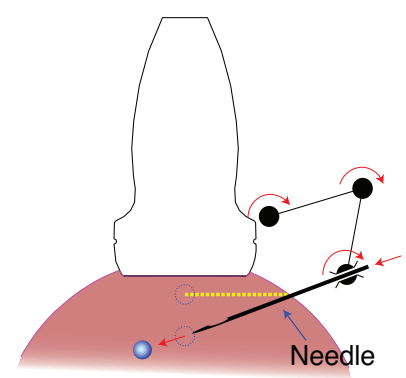

(d)

Figure 6.1: The approach to a robot-assisted US-guided biopsy on an MR-detected lesion: (a) Robotic manipulator approaches breast. In the US plane (b), the target moves upon positioning the US probe (c) and inserting the needle (d).

\subsection{Robot-assisted US-guided biopsy}

Figure 6.1 shows the approach for a robot-assisted US-guided biopsy on an MR-detected lesion. A patient is positioned in prone position over a robotic serial manipulator carrying an end-effector (EE) equipped with a US probe and a 3-DOFs actuated needle guide [170]. The guide aims the needle towards a target within the US plane. The lesion's position relative to the robot is known by registration of the MRI data with the patient by, e.g., a camera scan. A desired US plane through the lesion is defined, and a contact point of the US probe with the skin is derived such that the target is in the field of view (FOV) (Figure 6.1 (a)). The robot is aligned with the desired US plane (Figure 6.1(b)) and approaches the breast. During probe positioning (Figure 6.1.(c)) and needle insertion (Figure 6.1 d) ), the target displaces from its original estimated position. 
These two phases of the biopsy, including the compensation for the target displacement, will be discussed in the following sections.

\subsubsection{Probe positioning}

The probe positioning phase focuses on aligning the EE with the desired US plane, measuring the instant of contact and tracking tissue deformations. Additionally, it assures adequate acoustic coupling between the US probe and the skin in its final position. The probe's position and orientation remain static during needle insertion.

\section{Determining the contact position}

A triangular mesh, which describes the skin surface of the breast, and the target position, $p_{\mathrm{t}}$, are extracted from the preoperative data (Figure 6.1(a) and (b)). The desired US-plane, $A$, is constrained by the target position such that $p_{\mathrm{t}} \in A$. The orientation of $A$ is based on the radiologist's input, who could, e.g., prefer to align $A$ with the coronal plane. Possible contact positions are extracted by calculating the intersection of $A$ with the surface model. The radiologist also chooses the initial contact position. The robotic manipulator aligns the $\mathrm{x}-$ and z-axis of the EE frame, $\Psi_{\mathrm{ee}}$ in Figure 6.1, with $A$. It is assumed that if the contact point of the probe with the skin and the target location are coincident with the z-axis of $\Psi_{\text {ee }}$, the lesion will be in the FOV upon contact. Thus, the target coordinates can be associated with a pixel in the US image at the moment of contact.

\section{Acquiring contact}

The system diagram for acquiring contact is shown in Figure 6.2 Confidence maps are utilized to estimate the contact moment and gain acoustic coupling with the breast. The confidence map, $C$, represents the per-pixel confidence in the corresponding US image, $U$. The pixels of an image are located in a matrix $\Omega^{n \times m}$. An acquired US image, $U: \Omega \rightarrow[0,1]$, is associated with a confidence map, $C: \Omega \rightarrow[0,1]$. The map, $f: U \rightarrow C$, is solved as a random walk equilibrium that respects physical constraints specific to US. Furthermore, the top row of a confidence map is defined as 1 , and the bottom row is defined as 0 [172]. Confidence maps emphasize shadowed and attenuated regions and are useful to estimate how the probe is in contact with the skin. As shown in Figure 6.2 partial contact transfers to a high confidence region in the middle of the confidence map. The mean confidence, $C_{\text {mean }}$, correlates with the contact area of the US probe with the skin and is defined as

$$
C_{\text {mean }}=\frac{1}{n \cdot m} \sum_{(u, v) \in \Omega} C(u, v) .
$$




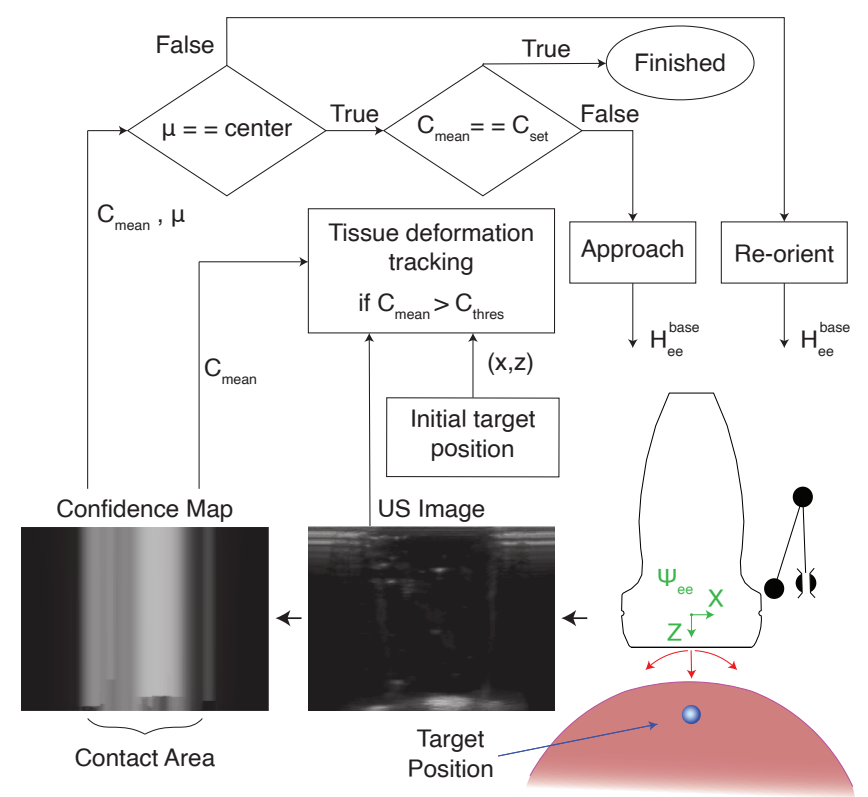

Figure 6.2: System diagram of the probe positioning phase. The features of the confidence map are used to adjust the probe position and to start deformation tracking. The US image is used to track the tissue deformation. The process stops if complete contact is achieved.

The controller continuously evaluates $C_{\text {mean }}$ during probe positioning. Figure 6.2 shows that a threshold value of the mean confidence, $C_{\text {thres }}$, is defined, which indicates the first contact and the start of tissue deformation tracking. Additionally, $C_{\text {set }}$ is defined as the mean confidence for which the probe has appropriate acoustic coupling with the breast.

Furthermore, the weighted barycentre, $\mu$, of the confidence indicates the location of contact with the probe and is defined as

$$
\begin{aligned}
& \mu_{u}=\frac{1}{C_{\Omega}} \sum_{(u, v) \in \Omega} u \cdot C(u, v), \\
& \mu_{v}=\frac{1}{C_{\Omega}} \sum_{(u, v) \in \Omega} v \cdot C(u, v),
\end{aligned}
$$

with $C_{\Omega}=\sum_{(i, j) \in \Omega} C(i, j)$ the total confidence. The pixel indices $\mu_{i}$ and $\mu_{j}$ correspond to EE coordinates $\mu_{z}$ and $\mu_{x}$, respectively. If these coordinates are located off-centre, the probe contact is off-centre and thus the target may not be in the FOV. Therefore, the probe should be rotated around the target by $\theta_{\mathrm{r}}=\arctan \frac{\mu_{x}}{\mu_{z}}$ radians. This movement is indicated in Figure 6.2. The new 
desired position relative to the current position expressed in $\Psi_{\mathrm{ee}}$ is given by

$$
\boldsymbol{H}_{\mathrm{d}}^{\mathrm{c}}=\left[\begin{array}{cc}
\boldsymbol{I}^{3 \times 3} & -d_{\mathrm{t}} \hat{\boldsymbol{z}} \\
\mathbf{0}^{1 \times 3} & 1
\end{array}\right]\left[\begin{array}{cc}
\operatorname{Rot}_{y}\left(\theta_{r}\right)^{3 \times 3} & \mathbf{0}^{3 \times 1} \\
\mathbf{0}^{1 \times 3} & 1
\end{array}\right]\left[\begin{array}{cc}
\boldsymbol{I}^{3 \times 3} & d_{\mathrm{t}} \hat{\boldsymbol{z}} \\
\mathbf{0}^{1 \times 3} & 1
\end{array}\right]
$$

where $\boldsymbol{I}$ is the identity matrix, $\hat{z}$ the unit vector in z-direction and $d_{\mathrm{t}}$ is the distance between the EE and the target. Once the barycentre is located in the centre, the robot approaches the breast until the mean confidence matches $C_{\text {set }}$.

\section{Tissue deformation tracking}

It is assumed that no significant deformation has taken place when $C_{\text {mean }}=C_{\text {thres }}$. Thus, the target, $p_{\mathrm{t}}$, still has its preoperatively defined position. Therefore, the target coordinates $(z, x)$ expressed in $\Psi_{\mathrm{ee}}$, as obtained from the preoperative data, can be mapped on a pixel $(i, j)$ of the US image $U$. The probe continues to move to acquire acoustic coupling. This motion compresses the tissue and thus moves the target from its original position. Since the target may not be visible, optical flow is used to track this motion. The popular Lucas-Kanade method is used [187] to track the movement of brightness patterns of the target location. This method assumes that the inter-frame movements are small and the same for a small window of pixels with the target at its centre. A pyramidal implementation of the algorithm is utilized to make the target tracking more robust 188. The algorithm outputs an updated position of the expected target location per acquired US image. The same tissue tracking algorithm is also utilized during needle insertion.

\subsubsection{Needle insertion}

After probe positioning, the radiologist starts inserting the needle. The system diagram is shown in Figure 6.3. The initial target position is the position of the target determined after probe positioning. The needle may displace the target due to tissue-needle interaction, and thus, the US images are evaluated to update the target position. Furthermore, the actual needle trajectory may differ from the one derived with the forward kinematics due to needle bending. Therefore, the system relies on a needle detection algorithm also.

\section{Needle trajectory}

As shown in Figure 6.3 the desired needle trajectory, expressed in the needle guide frame $\Psi_{\mathrm{ng}}$, is defined by the insertion point $p_{\mathrm{i}}=\left(x_{\mathrm{i}}, y_{\mathrm{i}}\right)$ and the target position $p_{\mathrm{t}}=\left(x_{\mathrm{t}}, y_{\mathrm{t}}\right)$ as

$$
y-y_{\mathrm{t}}=\frac{y_{\mathrm{i}}-y_{\mathrm{t}}}{x_{\mathrm{i}}-x_{\mathrm{t}}}\left(x-x_{\mathrm{t}}\right) .
$$




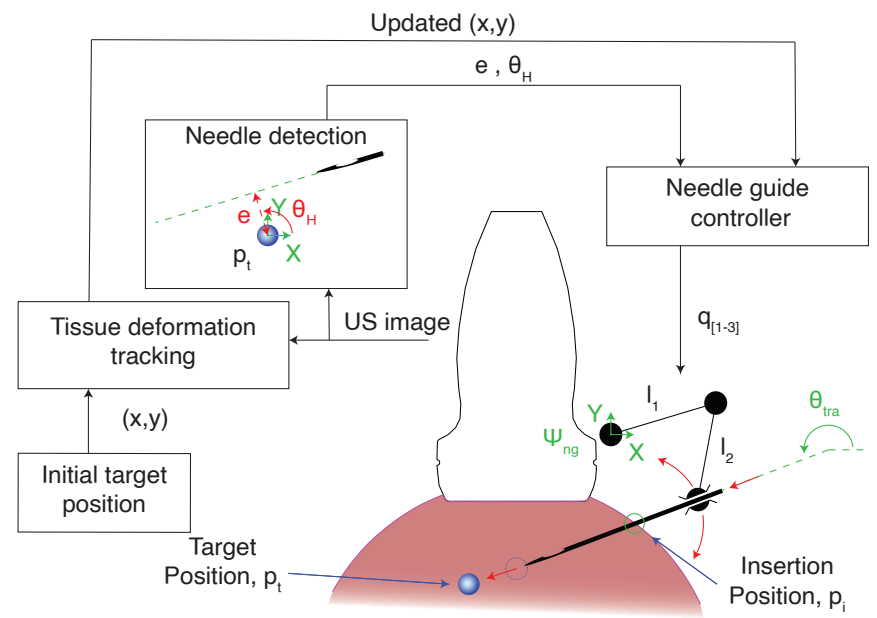

Figure 6.3: System diagram of the needle insertion phase. The controller adjusts the needle guide based on optical flow and needle detection. The insertion position remains constant, such that no stress is exerted on the skin.

The radiologist chooses the insertion position, which lays on the intersection of plane $A$ with the surface model of the breast. This position is kept constant throughout the needle insertion, while the target position is updated according to the tissue motions. This way, the center of motion is a point on the skin, and thus the needle will not cause any stress in this position. This approach resembles the way a radiologist would manually manipulate the needle.

\section{Needle detection}

The needle detection is broken down into several steps. First, Canny edge detection is applied to the US image [189]. Canny edge detection consists of the following processing steps: the application of a Gaussian filter to reduce the noise, finding the intensity gradients of the image along the $\mathrm{x}$ - and $\mathrm{y}$-axis, finding the sharpest edges, applying a double threshold to remove edge pixels caused by noise and finishing the edges by connecting the stronger edges with weaker ones. In the resulting image, the needle trajectory is found with the Hough transform. The error between the needle trajectory and the target is expressed as

$$
e=x \cos \theta_{\mathrm{H}}+y \sin \theta_{\mathrm{H}},
$$

with $e$ the shortest distance between the target and the trajectory and $\theta_{\mathrm{H}}$ the angle between the $\mathrm{x}$-axis and the normal connecting the target and the trajectory, as presented in see Figure 6.3 190]. The target, $p_{\mathrm{t}}$, is taken as the origin.

A controller adjusts the needle trajectory virtually moving the target position, 
$p_{\mathrm{t}}$, in the direction opposite to the normal defined by $e$ and $\theta_{\mathrm{H}}$. The offset to the needle trajectory remains constant if no needle is detected in the image.

\section{Needle guide position}

The needle guide's position, $p_{\text {ng }}$, is located at a constant distance, $d_{\mathrm{ng}}$, of the insertion point, $p_{\mathrm{i}}$, such that

$$
p_{\mathrm{ng}}=\left[\begin{array}{l}
x_{\mathrm{ng}} \\
y_{\mathrm{ng}}
\end{array}\right]=p_{\mathrm{i}}-\left[\begin{array}{l}
d_{\mathrm{ng}} \sin \theta_{\mathrm{tra}} \\
d_{\mathrm{ng}} \cos \theta_{\mathrm{tra}}
\end{array}\right],
$$

where $\theta_{\text {tra }}$ is the angle of the needle trajectory with the x-axis of $\Psi_{\text {ng }}$ as shown in Figure 6.3. The guide's joint positions are acquired via the inverse kinematics of the $2 \mathrm{D}$ planar system

$$
\begin{array}{ll}
q_{1}=\arctan 2(y, x) \pm \beta, & \beta=\cos ^{-1}\left(\frac{r^{2}+l_{1}^{2}-l_{2}^{2}}{2 l_{1} r}\right), \\
q_{2}=\pi \pm \alpha, & \alpha=\cos ^{-1}\left(\frac{l_{1}^{2}+l_{2}^{2}-r^{2}}{2 l_{1} l_{2}}\right) \\
q_{3}=\theta_{\text {tra }}-q_{1}-q_{2}, &
\end{array}
$$

in which $r=\sqrt{x_{\mathrm{ng}}^{2}+y_{\mathrm{ng}}^{2}}, l_{1}$ and $l_{2}$ are the lengths of link 1 and 2 , respectively, $q_{i}$ indicates the joint position of the i-th joint, and the signs for $\alpha$ and $\beta$ should agree 133 .

\subsection{Experimental validation}

\subsubsection{Experimental setup}

The setup (Figure 6.4) consists of a 7-DOFs robotic manipulator (KUKA Med 7 R800, KUKA GmbH, Germany) to which the EE is connected. The EE holds a VF13-5 linear US probe (Siemens AG, Germany) and includes a 3-DOFs needle guide. The transformation of both the transducer and the needle guide with respect to the flange is retrieved from the computer-aided design (CAD) file of the EE. The US probe is connected to an X300 US system (Siemens AG, Germany) which streams the US images with an update rate of $24 \mathrm{~Hz}$ to a workstation via a capture card (Pro Capture DVI HD, Magewell, China). The workstation communicates with the manipulator and the EE via the fast research interface and serial communication.

A phantom with a simplified breast shape, such that the deformations occurring during the procedure remain in-plane, is constructed with a polyvinyl chloride plastisol (PVCP)/Plasticizer mixture (Bricoleurre, France). The breast's 


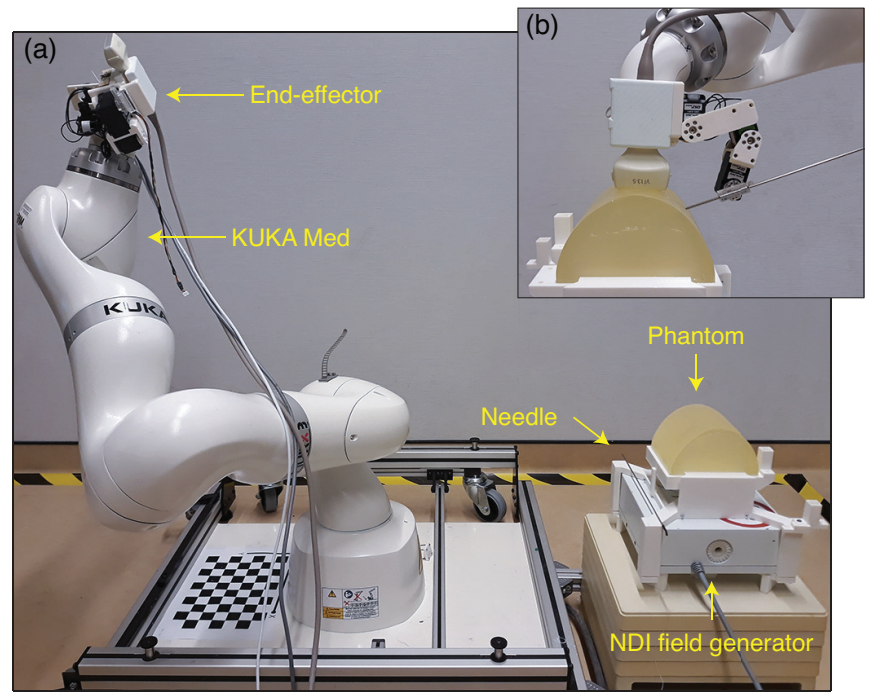

Figure 6.4: The robotic setup. (a) System overview. The KUKA LBR Med 7 with the end-effector attached, the NDI field generator, the tracked needle and the breast phantom are indicated. (b) Close-up of the EE with the needle inserted in the phantom.

skin is mimicked by a stiff outer layer of approximately $10 \mathrm{~mm}(100 \% / 0 \%)$, and the fatty tissue by a softer inner structure $(70 \% / 30 \%)$. While the skin layer is expected to have a comparable stiffness to actual skin, the inner structure may be up to ten times stiffer than actual fatty tissue 182 184. 1 wt.\% silica powder is added to both mixtures to increase scattering.

The phantom was placed on top of and registered with an Aurora tracker (Northern Digital Inc., Canada). An electromagnetic (EM) tracker (part nr: 610090, Northern Digital Inc., Canada) was placed inside the phantom at a depth of approximately $25 \mathrm{~mm}$ to function as a lesion with zero volume and a known location. The robot is registered with the Aurora tracker such that the initial lesion location relative to the robot is known. A custom biopsy needle was produced utilizing a metal tube with an outer diameter of $2 \mathrm{~mm}$ and equipped with an EM tracker (part nr: 610059). The experiments were performed in supine position since the bed interferes with the NDI equipment, but usually, the procedure is performed in prone position. The desired contact position was based on the current target position and the CAD file of the phantom. The Euclidean distance between the needle tip and the target determines the accuracy of the needle insertion experiments. This distance is retrieved by recording both sensor positions after insertion. Furthermore, the normal distance is determined, which is the shortest distance between the target and the needle trajectory. 

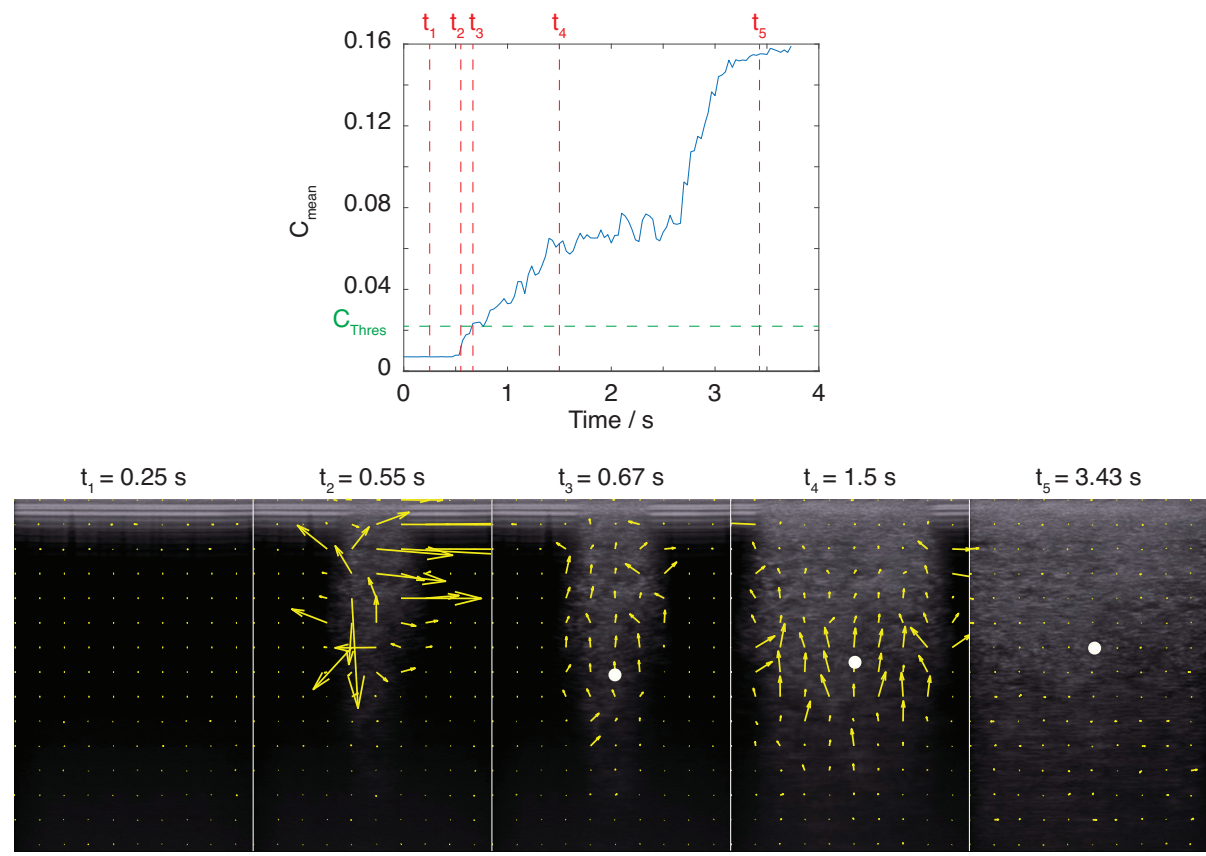

Figure 6.5: Graph showing the average confidence while approaching the breast and the corresponding US images for $t_{[1-5]}$. The yellow arrows indicate the optical flow field. The white dot is the tracked target starting from $C_{\text {mean }}=C_{\text {thres }}$.

\subsubsection{Experiments}

Three experiments were conducted. The first experiment determines the accuracy of the estimated target location with respect to the actual target location after probe positioning. The robot starts from its home position, aligns the EE with the indicated US plane, and brings the probe in contact with the phantom. The second experiment determines the in-plane accuracy of the needle placement. This experiment was performed with and without the needle detection activated. The needle is unlikely to bend during this experiment due to the soft phantom and stiff needle. Therefore, uniformly distributed errors of $\pm 0.08, \pm 0.06$ and \pm 0.03 rad were added to the initial setpoints of joint one, two and three, respectively. The third experiment determines the accuracy of the whole workflow. Each experiment was performed ten times, and averaging was applied.

\subsubsection{Results}

Figure 6.5 presents the mean confidence during an approach to the phantom accompanied with US images and their corresponding optical flow profiles at 
times $t_{[1-5]}$. The images taken at $t_{1}$ and $t_{2}$ show that the optical flow profile does not follow the deformation. However, at $t_{3}$ - when $C_{\text {mean }}=C_{\text {thres }}$ and $t_{4}$, the profile in the center of the image matches the deformation and the target, shown in white, moves along. In Table 6.1, the error between the tracked target position and the actual target position is stated for the initial position, when $C_{\text {mean }}=C_{\text {thres }}$ and the final position, when $C_{\text {mean }}=C_{\text {set }}$. It shows that the initial error is in the millimeter range, indicating that the target to robot registration has millimeter accuracy. Furthermore, it seems that, based on this data, a larger target displacement does not necessarily imply a larger error. The target displacement is the largest in the $\mathrm{z}$-direction, whereas the error in the $\mathrm{z}$-direction is not. Instead, the $\mathrm{x}$-direction has the largest error because the tracked position sometimes follows the expanding region, indicated by the horizontal arrows of the optical flow profiles shown in Figure 6.5 at $t_{3}$ and $t_{4}$.

Table 6.1: Mean absolute error between the estimated lesion position and the actual lesion position initially, when $C_{\text {mean }}=C_{\text {thres }}$, and finally, when $C_{\text {mean }}=C_{\text {set }}$, and the target displacement during the procedure.

\begin{tabular}{|c|c|c|c|c|}
\hline \multirow{4}{*}{ Error } & & \multirow{2}{*}{$\frac{d_{x} \text { (max.) }}{\mathrm{mm}}$} & \multirow{2}{*}{$\frac{d_{y} \text { (max.) }}{\mathrm{mm}}$} & \multirow{2}{*}{$\frac{d_{z}(\max .)}{\mathrm{mm}}$} \\
\hline & & & & \\
\hline & Initial & $1.03(1.28)$ & $0.59(1.82)$ & $1.23(1.71)$ \\
\hline & Final & $2.12(3.69)$ & $0.80(1.98)$ & $0.97(3.05)$ \\
\hline \multicolumn{2}{|c|}{ Target displacement } & $0.53(2.47)$ & 0.88 (2.99) & $2.35(8.61)$ \\
\hline
\end{tabular}

Table 6.2: Mean absolute distance between the needle tip and NDI target after needle insertion in $x, y$ and $z$ direction while the needle detection was switched off $(\boldsymbol{X})$ and on $(\boldsymbol{})$.

\begin{tabular}{|c|c|c|c|}
\hline & $d_{x}(\max )$. & $d_{y}(\max )$. & $d_{z}(\max )$. \\
\hline Needle detection & $\mathrm{mm}$ & $\mathrm{mm}$ & $\mathrm{mm}$ \\
\hline$x$ & $0.72(1.44)$ & $0.76(2.05)$ & $0.76(2.15)$ \\
\hline$\checkmark$ & $1.81(3.12)$ & $1.61(4.60)$ & $1.54(3.14)$ \\
\hline
\end{tabular}

Table 6.3: The mean absolute distance of the needle tip, and the normal distance of the needle trajectory with respect to NDI target, after completing the procedure in which the probe is placed and the needle is inserted. Additionally, the target displacement is noted.

\begin{tabular}{|c|c|c|c|c|}
\hline & $d_{x}(\max )$. & $d_{y}(\max )$. & $d_{z}(\max )$. & $d_{\text {norm }}(\max )$. \\
\hline & $\mathrm{mm}$ & $\mathrm{mm}$ & $\mathrm{mm}$ & $\mathrm{mm}$ \\
\hline Total error & $1.15(2.84)$ & $1.31(3.53)$ & $3.47(5.05)$ & $2.89(4.88)$ \\
\hline Target displ. & $0.84(2.52)$ & $0.93(3.32)$ & $2.66(9.13)$ & \\
\hline
\end{tabular}




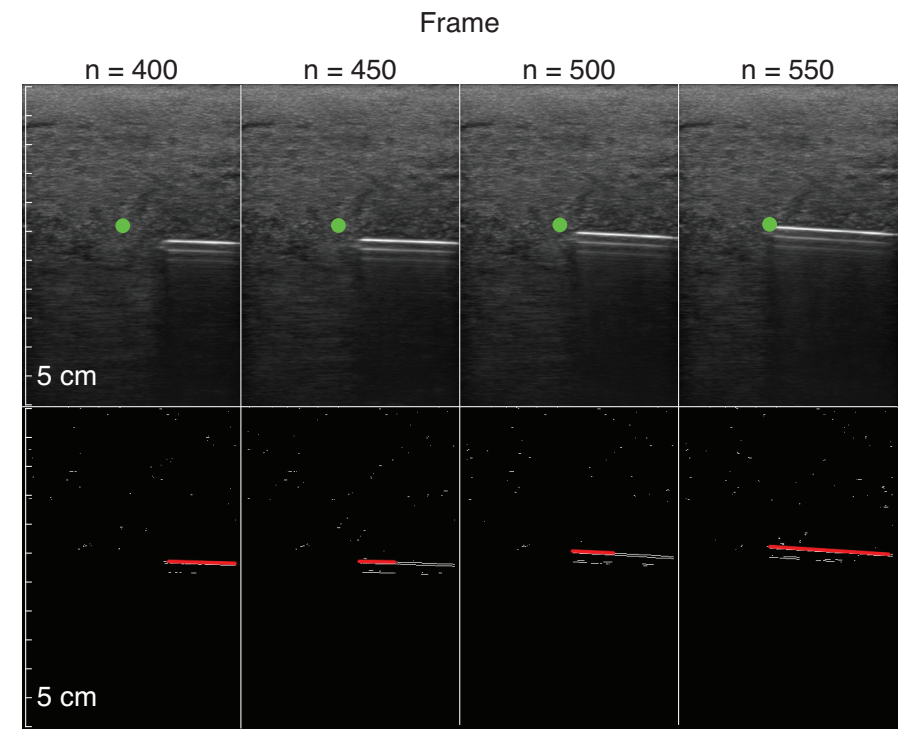

Figure 6.6: Image sequence showing the needle trajectory adjustment based on needle detection. Both the US image with the tracked point and the processed image with the detected needle are shown. The frame rate was $20 \mathrm{~Hz}$.

Table 6.2 shows that enabling needle detection makes the needle placement less accurate. The detection algorithm marks the top edge - not the core of the needle, which is subsequently aligned with the target (Figure 6.6). Thus, the core of the needle is off by the needle radius.

Table 6.3 presents the accuracy of the entire workflow, in which the robotic manipulator first positions the US probe to view the target and then guides the needle towards the target. At this moment, the error is not directly relatable to the errors found in Table 6.1 and Table 6.2 Additional experiments may find this relation.

\subsection{Discussion}

This study demonstrated an approach to a robot-assisted US-guided biopsy on MR-detected lesions, which may be hard to target otherwise. The overall accuracy, as presented in Table 6.3 indicates that it is feasible to target lesions with a radius down to $3 \mathrm{~mm}$. This accuracy is acceptable in breast cancer diagnostics and similar to equivalent experiments performed in the cited studies (1.1 to $3.44 \mathrm{~mm}$ ) 83,98 . The accuracy could be increased by improving the registration between the phantom and the robot, which is currently in the millimeter range. Other factors influencing the accuracy are the target tracking, 
the calibration of the US probe and the needle guide with the robot flange, and the accuracy of the needle guide itself.

The main limitation of this study is the assumption that the lesion remains in-plane. This assumption was correct for the performed experiments since the phantom and the applied forces were symmetric. However, in real-life situations, the tumor may move out-of-plane due to asymmetry in the applied pressure and boundary conditions. Nonetheless, the system can easily be extended to 3D by switching to a 3D US probe. Both confidence maps and deformation tracking have previously been applied to 3D US. Additionally, out-of-plane motions may be detected from the B-mode images by taking the divergence of the optical flow field. In future steps, both simulation and feedback algorithms may be combined to account for deformations that the current system cannot.

Furthermore, the deformation of breast tissue is more significant than of phantom material: the structure is less constrained, the material softer, and although positioned in prone position, the patient may move. Therefore, the manipulator may need to adjust the probe position as well as the needle guide during needle insertion to retain acoustic coupling. Impedance control of the needle guide may provide compliance for small patient movements but affects accuracy. Also, a safety release mechanism may be needed in the clinical environment to release the needle if the patient inadvertently moves more than expected.

This work focused on integrating US feedback to estimate probe contact and compensate for deformations. It showed that confidence maps are suitable for evaluating probe contact and acquiring acoustic coupling with the phantom. The confidence map has advantages over force feedback since variations in breast stiffness will not influence the image quality while deformations are kept to a minimum. Figure 6.5 shows how the confidence map serves to start the tissue tracking. The optical flow profile shows the tissue compression in the region in contact, whereas the edges show some vectors pointing sideways, indicating the expanding contact region. Sometimes, the tracked target moved along horizontally with this region, leading to a more significant error along this direction. This error can be prevented by explicitly evaluating the confidence around the target area, by setting $C_{\text {thres }}$ to a higher value, and by having a more accurate registration between the target and the robot. The optical flow was also successfully applied during needle insertion. Table 6.2 shows that the in-plane accuracy of the needle placement is currently more accurate without needle detection. The implemented needle detection algorithm detects and aligns the top edge of the needle with the target, whereas the forward kinematics considers the center of the needle. The needle detection will be more accurate by incorporating the needle radius in the error, $e$, in Equation (6.5).

Overall, the system shows some promising features: the robot-assisted biopsy minimizes the MR-time since the biopsy takes place under US guidance, the time per biopsy is less because the system automatically navigates to the correct 
US plane, and the radiologist gains robotic accuracy, while he is in control like in the conventional procedure. Thus, the radiologist can still respond appropriately to haptic or patient feedback.

\subsection{Conclusion}

A robotic workflow was introduced to assist the radiologist in accurately performing US-guided biopsies on MR-detected lesions. Experiments show that the controller can use confidence maps to estimate first probe contact and optical flow to track tissue deformation in areas with high confidence with millimeter accuracy. The needle detection algorithm reduces errors in the initial direction of the needle but is, at this point, less accurate than using only the encoders of the needle guide. The proposed workflow has millimeter accuracy in the current setting. 


\section{MR Safe RGB Spectrophotometer-based Single Fiber Position Sensor}

Adapted from:

M. K. Welleweerd, L. Abelmann, S. Stramigioli, and F. J. Siepel, "MR Safe

RGB Spectrophotometer-based Single Fiber Position Sensor," In preparation, 


\begin{abstract}
Traditionally, magnetic resonance (MR) safe robots have fiber-based optic position sensors which are fast and sensitive. Often, these sensors use so-called quadrature modulation to resolve the magnitude and direction of displacement. Two monochromatic optical channels are needed for this modulation, often supplemented by a third channel to obtain the position via a calibration movement. This paper explores whether additional wavelengths in the light source can add more functionality to a single optical channel. To this end, a simplified spectrophotometer is designed that senses the reflection of three wavelengths (red, green, blue) from a patterned paper strip in front of the detector. The patterned paper is attached to the translator and selectively absorbs each of the three wavelengths. A single optical channel can resolve the magnitude and direction of displacement if two colors are printed in a quadrature configuration. The root mean square (RMS) error of displacement measurements is down to $(6.2 \pm 4.6) \mu \mathrm{m}$ if the higher harmonics of the calibration are taken into account. Superposing a gradient of the third color over the quadrature pattern shows the potential also to resolve the absolute position of the translator. The achieved RMS error of $(157.9 \pm 657.4) \mu \mathrm{m}$ can be further improved by removing nonlinearities in the printed pattern. Overall, a minor adjustment - replacing a monochromatic with a trichromatic light source - enables the integration of more functionality in one optic channel and more minimalistic sensing solutions.
\end{abstract}




\subsection{Introduction}

magnetic resonance imaging (MRI) plays an ever more prominent role in health care. The modality is used for detection and diagnostics as well as for interventions. Currently, MRI is the most sensitive imaging modality, but the constraints of the workspace and the human operator are limiting the accuracy of interventional procedures. As a result, several robotic systems are making their way into the MRI bore to support the radiologists in more accurately performing the procedure. Examples of applications are neurosurgery and percutaneous interventions such as biopsies or tumor ablations in the liver, prostate, kidneys or breast 191.

The design requirements for in-bore robots are strenuous because of limited space and the necessity to be magnetic resonance (MR) safe. A device should pose no known hazards in all MRI environments to be MR safe, and thus, it should consist of nonconducting, nonmetallic and nonmagnetic materials [192]. Hence, the actuation methods of MR safe robots differ from traditional robotic actuators. Robots have been presented with piezoelectric, pneumatic, hydraulic, and tendon-driven actuation [115 118].

These MR safe robots need sensing mechanisms to reach their full potential, providing feedback for various control algorithms. Position control needs joint sensors to retrieve the current manipulator position; force feedback may be necessary to implement impedance control, haptic feedback in teleoperated solutions or to identify tissue types 193 . For a robot to be MR safe, all its sensing mechanisms should be MR safe as well.

Fiber-optic sensors are often considered when measuring various quantities (e.g., temperature, force, torque, strain and position) inside the MRI bore: they can be both sensitive and fast; the readout electronics can be placed outside the MRI room; the diagnostic accuracy of the MRI is unaltered; they are immune to the strong magnetic fields inside the MRI bore. A fiber-optic sensor's main component is the optic fiber, through which light is transmitted. This fiber generally consists of plastic or glass, with a high refractive index core and a low reflective index cladding. There are two types of optic fibers: single-mode and multi-mode. Single-mode fibers support only one propagation mode and preserve the coherence properties of light. Multi-mode fibers support multiple modes and have a large numerical aperture that allows for efficient light coupling. They are suitable for incoherent wide-angled light sources such as light-emitting diodes (LEDs). Two groups of fiber optic sensors can be distinguished: in intrinsic sensors, the optical fiber itself is the sensing element; in extrinsic sensors, the optical fiber only transmits the light while the measurand changes the light's characteristics [194].

The changeable characteristics of light are intensity, phase and wavelength 193. Intensity-modulated sensors rely on intensity change as a function of the measured quantity. In transmittance-based intensity-modulated sensors, the 
fibers face each other, whereas, in reflectance-based sensors, the light bounces off a surface before it reaches the receiving fiber. The transmitted light may be coupled in multiple receiving fibers for both transmissive and reflective sensors to achieve a differential measurement or sense multiple degrees of freedom. Sensors based on phase change measure the relative phase shift of two light beams. In a Fabry-Perot interferometer, the light propagates between two partially reflective mirrors. Multiple beams with varying optical path lengths generate destructive or constructive interference based on the length of the cavity. In the end, this leads to intensity modulation as well. Wavelength modulation is commonly achieved with a fiber Bragg grating (FBG). An FBG optical fiber has a periodic variation in the refractive index of the fiber core, with which a wavelength-specific mirror is obtained. A broadband light source is coupled with the fiber and the grating - and as a result, the reflected wavelength - changes due to the strain.

Fiber optic position or displacement sensors often employ intensity modulation and output binary or analog optical signals. Binary sensors make a distinction between two light intensities 195, 196]. Angular and linear position encoders may be based on the reflected intensity of a patterned black and white surface or a selectively milled surface 197. Additionally, the light beam can be cyclically interrupted by a patterned disk or slider [198]. Quadrature encoding can be used to indicate both the magnitude and direction of displacement, where two optical circuits measure two identical patterns with a ninety degrees phase shift relative to one another 198. The accuracy of these sensors mostly depends on the resolution of the encoder pattern. Currently, available sensors achieve a twelve to fourteen bit or $50 \mu \mathrm{m}$ accuracy for rotary and linear encoders, respectively 199, 200]. Analog optical sensors are the alternative. The intensity may be modulated by changing the distance of a reflective surface to the receiving fiber 201] or by varying the absorbance of light due to a changing color of the reflector [202, 203]. In work presented by Kwon et al. [202], an angular accuracy of $-1.46^{\circ}$ is obtained for a rotary encoder, whereas NELSON 203 achieves a positioning accuracy of $30 \mu \mathrm{m}$ utilizing a linear optical sensor. Both studies modify the absorbance of an LED with a printed pattern.

Traditionally, MR safe position sensors are designed using single-color LEDs and one optical fiber per LED. The integration of multi-color LEDs allows coupling multiple wavelengths in a single fiber. Thus, wavelength-dependent reflection and absorption spectra of the material passing the fiber can be measured. Instead of patterning the rotor or translator with a single color, as was previously done by Kwon et al. 202 and NELSON [203], a more complex pattern that selectively reflects one of the LED's wavelengths could be used.

This work investigates the advantages of integrating a multi-color LED combined with a multi-color pattern on a translator, compared to a single color LED combined with a single-color pattern. In section 7.2 the design of an optical detector that measures the reflectance of light off a printed paper strip is presented. This paper strip is easily fabricated with a multi-color printer. 


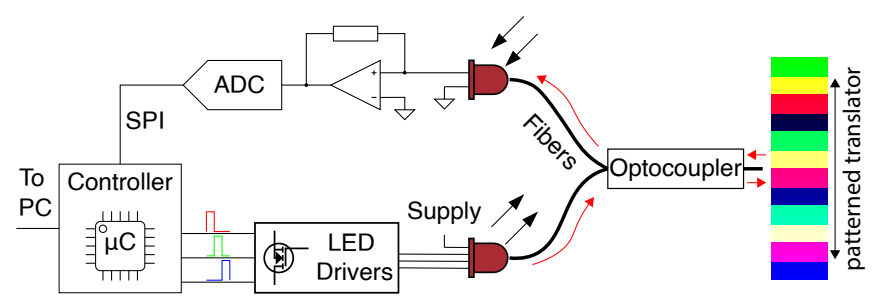

(a)

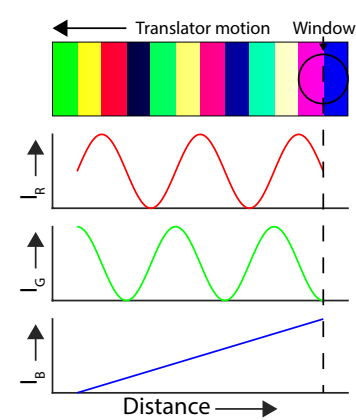

(b)

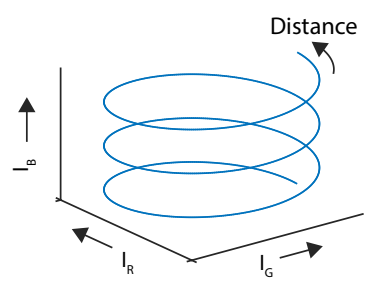

(c)

Figure 7.1: (a) A schematic overview of the measurement setup. A patterned translator moves in front of an optic fiber through which sequentially red, green and blue light is emitted. The reflection is quantized by a photodiode, a current to voltage converter and an analog-to-digital converter. The microcontroller communicates the result to a PC. (b) The optic fiber forms a circular window through which light is projected on the translator. The reflections are received through the same fiber and form a quadrature signal for the red and green channel, whereas the blue signal linearly increases. (c) Each position is uniquely represented by a color on a helical path through color-space through this pattern.

section 7.3 presents the implementation, an experimental setup to compare the position measured by our detector with the position measured by a vibrometer, and multiple experiments to compare the single-color LED performance compared to the multi-color LED performance. Section 7.4 presents the results. Section 7.5 and 7.6 discuss and conclude the presented work, respectively.

\subsection{Theory}

This section elaborates on the working principle of the spectrophotometer-based position sensor. Figure 7.1 shows an overview of the sensing principle. An optic fiber faces a translator with a color pattern (Figure 7.1(a)). Light is emitted from the fiber by a Red Green Blue (RGB) LED connected to this fiber via an optocoupler. The light reflects off the pattern back into the fiber and falls on a photodiode (PD) via the optocoupler. A current to voltage converter 
amplifies the signal, after which an analog-to-digital converter (ADC) digitizes it. By sequentially emitting red, green and blue light for $10 \mathrm{~ms}$ and digitizing the resulting voltage, the reflectance of the segment currently in front of the fiber is determined for three wavelengths. By choosing the colors on the bar smartly, the currently measured reflectance represents the bar's position relative to the fiber.

\subsubsection{Position encoding}

The light emitted by the LED, as well as the colors on the translator, consist of three components: red, green and blue. Assuming that the LED emits discrete wavelengths, which match the wavelengths reflected by the printed colors, the light can be selectively reflected by varying the intensity of red, green, and blue in the pattern. The position of the bar relative to the fiber can be determined by making specific reflectance patterns. In this work, two types of intensity modulation are considered: sinusoidal and linear. For both types, a simple model can predict the waveform of the measured light intensity while moving the translator along the fiber.

\section{Sinusoidal}

Sinusoidal modulation is obtained by alternatingly switching a color between the minimum and maximum intensity to achieve either maximum absorbance or maximum reflectance of the corresponding wavelength (Figure 7.2 (a)). The relative signal intensity during a transition from a patch with zero intensity to a patch with the maximum intensity of either red, green or blue is given by:

$$
\begin{aligned}
I_{i}(h) & =\frac{1}{\pi r^{2}} A(h) c_{i}, \quad \text { with: } \\
A(h) & =r^{2} \arccos \left(1-\frac{h}{r}\right)-(r-h) \sqrt{r^{2}-(r-h)^{2}} .
\end{aligned}
$$

Index $i$ can be $\mathrm{r}$, g or $\mathrm{b}$, referring to the colors red, green or blue, respectively. $I_{i} \in[0,1]$ is the relative signal intensity. $c_{i} \in[0,1]$ is the intensity of the colored patch, and can be left out if $c_{i}=1 . A(h)$ is the area of the color overlapping with the optical fiber. $h \in[0,2 r]$ is the displacement of the bar, $r$ is the radius of the fiber. Based on this model, if the patches' width equals the fiber diameter, a sinusoidal relative intensity $I_{i}$ would be expected while moving the pattern along the fiber. By laying two differently-colored patterns on top of each other with a $90^{\circ}$ phase shift, a quadrature signal is obtained, see Figure $7.1(\mathrm{~b}), I_{\mathrm{r}}$ and $I_{\mathrm{g}}$. 


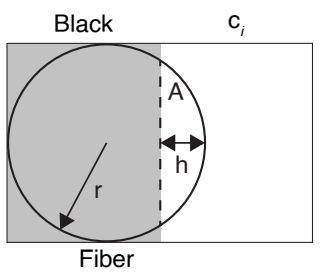

(a)

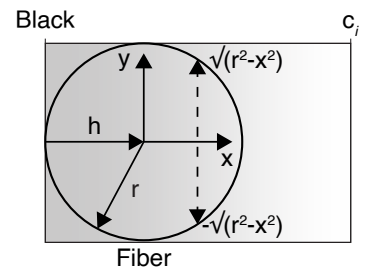

(b)

Figure 7.2: Two modulation schemes are used for position encoding. (a) Sinusoidal. The reflected intensity is a function of the overlap $A$ of the colored section with the circular optic fiber. (b) Linear. The reflected intensity is a function of the color intensity integrated over the area of the optic fiber.

\section{Linear}

Linear modulation is obtained by linearly increasing the color intensity over the length of the translator (Figure 7.2(b)). The currently reflected intensity depends on the average intensity of the printed color currently facing the fiber:

$$
\begin{aligned}
I_{i}(h) & =\frac{1}{\pi r^{2}} \int_{-r}^{r} \int_{-a}^{a} \frac{c_{i, \max }(h)+c_{i, \min }(h)}{2} d y d x \quad \text { with: } \\
a & =\sqrt{r^{2}-x^{2}}
\end{aligned}
$$

where the $x$-axis is aligned with the direction of movement and the gradient increase/decrease. $c_{i, \max }(h) \in[0,1]$ and $c_{i, \min }(h) \in[0,1]$ are the maximum and minimum intensity of the color in the section facing the fiber, respectively. $h \in[0, w]$ is the position of the fiber, with $w$ the total width of the translator. As such, the reflected intensity is linearly increasing while the bar is moving, see $I_{\mathrm{b}}$ in Figure 7.1(b).

Analog quadrature signals can have a high spatial resolution but measure displacement rather than position. Thus, they need a calibration step to obtain the position. By combining a quadrature signal, for instance, red and green, with a linear signal, for instance, blue, each position on the translator is uniquely represented by a combination of colors in a three-dimensional color space (Figure $7.1(\mathrm{c}))$.

\subsubsection{Position decoding}

If the intensity $I_{\mathrm{r}}$ of the red signal is plotted versus the intensity $I_{\mathrm{g}}$ of the green signal, and both signals would be perfect sinusoids, the signal would describe a circle. In this case, the current arctan of the signal represents the displacement of the bar relative to the fiber. The unwrap function maps jumps larger than $\pi$ on their $2 \pi$ complement by comparing the current angle with the previous measurement. The total displacement is then given by 


$$
\begin{aligned}
d_{i} & =\operatorname{unwrap}\left(\theta_{i}\right) \frac{l}{2 \pi}, \quad \text { with: } \\
\theta_{i} & =\arctan \left(\frac{I_{\mathrm{r}}}{I_{\mathrm{g}}}\right)
\end{aligned}
$$

With $d_{i}$ the displacement at the $i$-th measurement and $l$ the length of one period of the printed pattern. If at the first measurement, $d_{0}$ the position of the bar with respect to the fiber is known, this equation also gives the position.

Nevertheless, analog quadrature detectors have a common set of errors that limit obtainable precision and accuracy 204]. These are, for instance, imperfect quadrature, such that the phase shift is not precisely $90^{\circ}$, zero offset and unequal gain in the detector channels. Additionally, the waveform may be intrinsically different from a circle, such as a diamond shape in the case of a traditional rotary encoder disk. In this case, the result of Equation (7.3) has small, repetitive errors depending on the position along the waveform. A better estimate of the actual displacement can be obtained by analyzing the frequency components in the waveform with a calibration step 205. The Fourier series $F$ of the reflected intensity is given by

$$
F_{i}(x)=\sum_{n=1}^{N}\left(a_{i, n} \cos \left(\frac{2 \pi}{l} n x\right)+b_{i, n} \sin \left(\frac{2 \pi}{l} n x\right)\right),
$$

with index $i$ being $\mathrm{r}$, $\mathrm{g}$ or $\mathrm{b}$ for the red, green or blue channel, respectively. $a_{i}, n$ and $b_{i}, n$ are the amplitudes of the $n$-th $\cos$ and sin, respectively. $x$ is the position of the bar with respect to the optical fiber. These Fourier series can be obtained by a calibration step in which the translator is moved from one end to the other, while measuring the reflected intensities as well as the displacement using external measurement equipment. Now, to get a better estimate of the displacement during real-time measurements, the following optimization problem should be solved:

$$
\begin{aligned}
\hat{d}_{i}=\underset{x \in\left[b_{1}, b_{\mathrm{u}}\right]}{\arg \min } \underbrace{\left(F_{\mathrm{r}}(x)-I_{r}\right)^{2}+\left(F_{\mathrm{g}}(x)-I_{g}\right)^{2}}_{\text {quadrature encoding }}+ \\
\underbrace{\left(F_{\mathrm{b}}(x)-I_{b}\right)^{2}}_{\text {absolute }} .
\end{aligned}
$$

Where $\hat{d}_{i}$ is an improved estimation of the position on the $i$-th measurement, $b_{\mathrm{l}}$ and $b_{\mathrm{u}}$ are the lower and upper boundary of the search, respectively. Note that for a quadrature encoder, where the initial position at $i=0$ is known, only the first two terms of the equation should be optimized. In this case, a good initial guess for $x$ is $d_{i}$, and the boundaries can be chosen closely around $d_{i}$ for 
an efficient search. However, if the absolute position should be obtained, the third term should also be taken into account. In this case, a global minimum should be found, and $b_{1}$ and $b_{\mathrm{u}}$ should cover the entire range of the translator.

\subsection{Methods}

\subsubsection{Implementation}

\section{Light source}

Ideally, the wavelength of the light source is matched with the reflectance of the printed color. In traditional spectrophotometers, a full-spectrum Xenon flashlight generates the light, while a monochromator selects a specific wavelength. However, with LEDs, the wavelength is determined by the composition of the junction. Furthermore, the bandwidth of each wavelength is large $(25 \mathrm{~nm}$ compared to $5 \mathrm{~nm}$ for monochromators). The advantages of RGB LEDs are compactness, price, efficiency and availability. Additionally, three channels are sufficient to implement the previously designed patterns. Our device houses a relatively bright RGB LED with a non-diffuse housing to efficiently couple the light in the optical fiber (WP154A4SEJ3VBDZGC/CA, Kingbright Co., Taiwan).

\section{Detector}

A large-area PD is used to have a high sensitivity combined with a small form factor (VTB8441BH, Excelitas Technologies Corp., Canada). The diode is operated in photovoltaic mode, which means zero bias voltage. In this mode, the dark current, which is highly temperature-sensitive, is minimized. A one-stage opamp circuit amplifies the PD current. The low-noise opamp (OPA140, Texas Instruments Inc., USA) has a JFET input stage because this provides a low input current offset, which reduces DC errors and noise at the output. The output voltage of the amplifier stage is quantized by a 24-bit sigma-delta ADC (AD7195, Analog Devices Inc., USA). This ADC has an integrated temperature sensor to monitor the PCB's temperature.

\section{Fibers}

Standard Jacketed $1000 \mu \mathrm{m}$ polymethyl methacrylate (PMMA) core plastic fiber cables are used, connected to a $1 \times 2$ optical fiber splitter (IF-562, Industrial Fiber Optics Inc., USA). The jacket prevents ambient light from entering the setup, whereas the core diameter and material are ideally suited to be incorporated in a design without additional tooling. The light power coming from one side of the fiber splitter is equally divided over the two fibers on the other side, which 


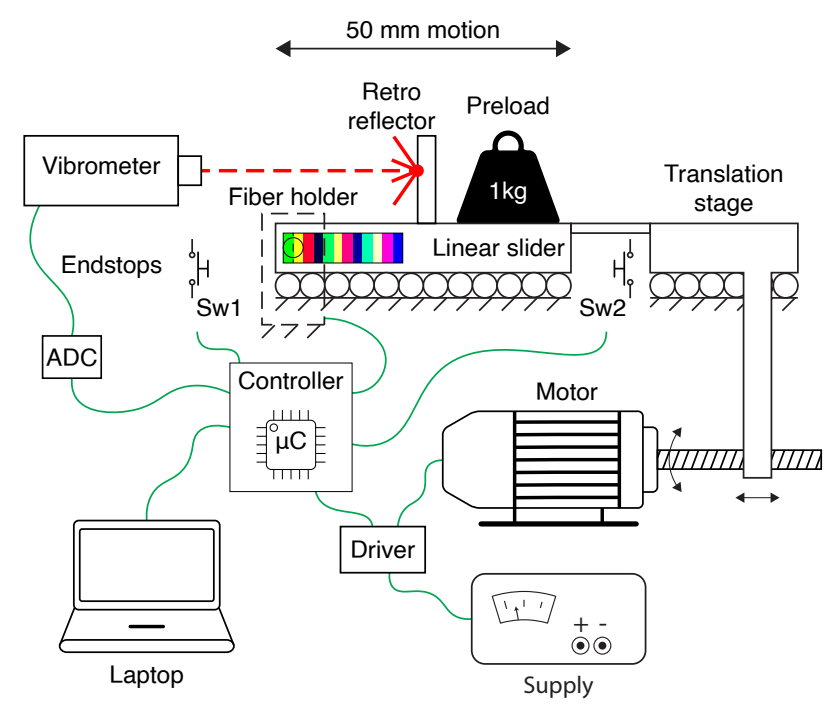

Figure 7.3: The setup for validating the displacement sensor.

effectively means that half of the reflected light reaches the detector. Plastic fiber optic connectors are used since they can be manually attached to the fiber and are MR safe (HFBR-4533Z, Broadcom Inc., USA). The fiber ends are polished for optimal light coupling utilizing a specially designed polishing kit (AFBR-4594Z, Broadcom Inc., USA).

\section{Printed patterns}

The patterns are printed with a printer (HP Z9, HP Inc., USA) that supports printing in chromatic red, green and blue and has a $2400 \times 1200$ dots per inch resolution. The extra colors and high resolution allow closely matching the designed colors and patterns. $l$ is chosen twice the optical fiber diameter to achieve optimal signal variation along the pattern.

\subsubsection{Experimental setup}

The experimental setup (Figure 7.3) consists of the optical detector as discussed in Section 7.2 and 7.3.1. The printed patterns are stuck to a linear slider with double-sided tape. The slider is connected to a linear translation stage. A DC motor, controlled with the microcontroller $(\mu \mathrm{C})$ via an H-bridge, turns the stage's actuator. A vibrometer (sensor head: OFV-505, controller: OFV-5000, Polytec $\mathrm{GmbH}$, Germany) measures the displacement with a resolution of $5120 \mathrm{\mu m} \mathrm{V}^{-1}$, which is represented by a voltage of $-8 \mathrm{~V}$ to $8 \mathrm{~V}$ on the output of the controller. 


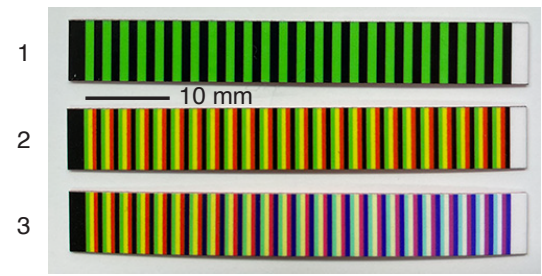

Figure 7.4: Pattern 1 has a sinusoidal modulation of green, pattern 2 has sinusoidal modulations of red and green with a $90^{\circ}$ phase-shift superposed, pattern 3 is pattern 2 with a linear modulation of blue superposed.

This voltage is scaled to $0 \mathrm{~V}$ to $3 \mathrm{~V}$ by a resistor network and digitized with a 16-bit ADC (ADS1115, Texas Instruments, USA) by the $\mu \mathrm{C}$. In each loop, the $\mu \mathrm{C}$ digitizes the red, green and blue channels as well as the vibrometer signal. Subsequently, the linear stage is moved for $100 \mathrm{~ms}$, and the next measurement is taken. The $\mu \mathrm{C}$ automatically moves the translation stage up and down over the $50 \mathrm{~mm}$ range of the translation stage based on two limit switches.

\subsubsection{Experiments}

Three patterns were measured and compared (Figure 7.4). (1) A monochromatic pattern to obtain sinusoidal modulation by alternating green with black. Green was chosen, as this channel gave the highest signal intensity. (2) The sinusoidal modulation of green and red are $90^{\circ}$ phase-shifted and superposed to obtain quadrature encoding. (3) The quadrature encoding of (2) is supplemented with a linear modulation of blue. As such, the three patterns modulate the reflectance of one, two or three wavelengths, and the value of adding wavelengths becomes clear. The patterns have 24 periods followed by a section of $2 \mathrm{~mm}$ of black or white to indicate the end, which amounts to samples of $52 \mathrm{~mm}$.

We waited until the board had a steady temperature to ensure stable measurements using the ADC's integrated temperature sensor. Each pattern is traversed fifty times. The uneven traversals are used for calibration, whereas the even ones are used for validation. Calibration is done by taking the average value of the calibration measurements at each position and approximating this curve with Equation (7.4) with $N=200$.

\subsection{Results}

Figure 7.5 shows the data for 25 traversals of each pattern. On average, each traversal contains $1.15 \times 10^{3}$ measurements. We analyze the section -22.5 to $22.5 \mathrm{~mm}$, since, outside this range, the high peaks on the left of the graphs show that the end of the pattern is reached. 


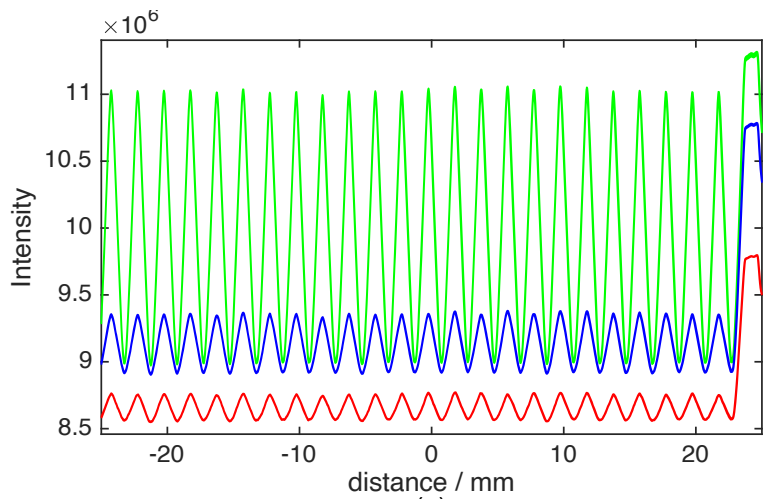

(a)

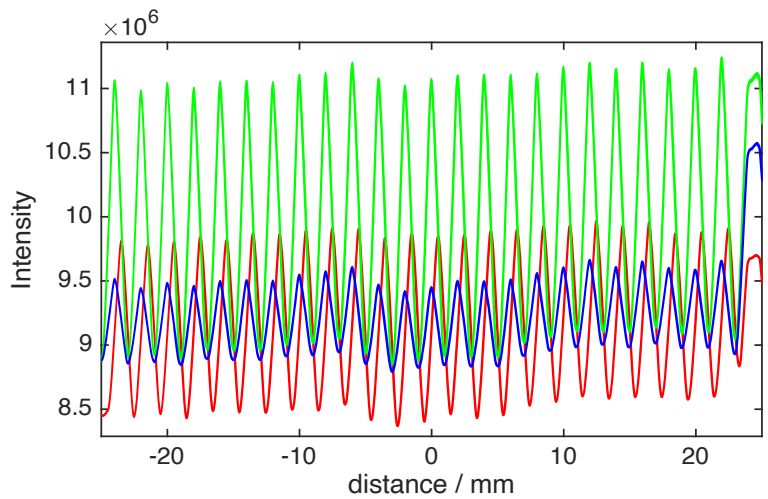

(b)

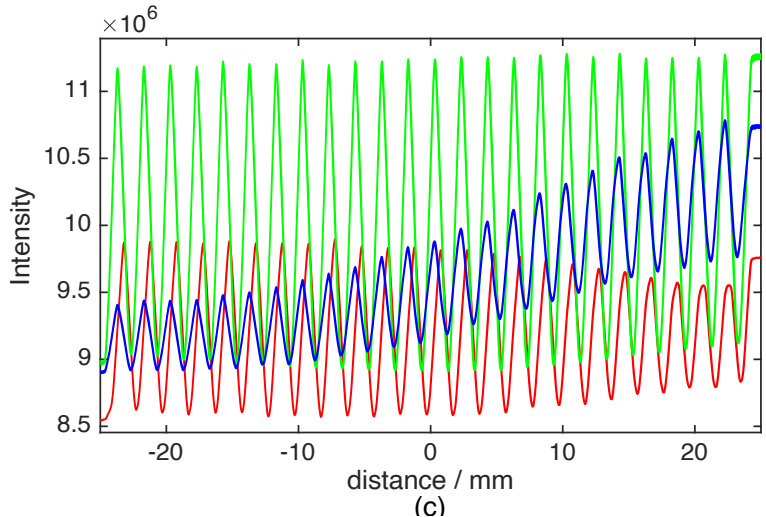

Figure 7.5: From top to bottom the response of the red, green and blue channel for pattern 1, 2 and 3. 


\subsubsection{Pattern 1}

Figure 7.5(a) shows the data for pattern 1. It shows that also the red and blue wavelength respond to the green color. The maximum intensity changes due to the pattern are $\Delta I_{\mathrm{r}}=2.25 \times 10^{5}, \Delta I_{\mathrm{g}}=2.09 \times 10^{6}, \Delta I_{\mathrm{b}}=4.71 \times 10^{5}$.

\subsubsection{Pattern 2}

Figure 7.5 (b) shows the data for pattern 2. The maximum signal changes due to the pattern are $\Delta I_{\mathrm{r}}=1.60 \times 10^{6}, \Delta I_{\mathrm{g}}=2.40 \times 10^{6}$ and $\Delta I_{\mathrm{b}}=8.78 \times 10^{5}$. The amplitude of $I_{\mathrm{b}}$ is higher than in pattern 1, although the blue color is not printed. Due to the phase difference between $I_{\mathrm{g}}$ and $I_{\mathrm{r}}$, the direction of movement can be resolved.

Figure 7.6 (a) shows displacement measurements based on $I_{\mathrm{r}}$ and $I_{\mathrm{g}}$, assuming the initial position $d_{0}$ was known. We used both Equation (7.3) and (7.5) to estimate the displacement, indicated by $d$ and $\hat{d}$, respectively. The root mean square (RMS) error of $d$ was $(47.3 \pm 28.9) \mu \mathrm{m}$, whereas the RMS error of $\hat{d}$ was $(7.6 \pm 7.2) \mu \mathrm{m}$. Optimization using Matlab's fmincon algorithm took $23.9 \mathrm{~ms}$ per measurement. For $\hat{d}$, the first and last fifty samples of each traversal are skipped because the Fourier series did not accurately approximate the calibration signal in these sections due to window effects.

\subsubsection{Pattern 3}

Figure 7.5(c) shows the calibration data for pattern 3. The intensity variations are $\Delta I_{\mathrm{r}}=1.35 \times 10^{6}, \Delta I_{\mathrm{g}}=2.37 \times 10^{6}$ and $\Delta I_{\mathrm{b}}=1.89 \times 10^{6}$. It is shown that the amplitude of the red channel decreases as the intensity of the blue channel increases. Furthermore, $I_{\mathrm{b}}$ does not show the intended linear intensity variation over the length of the pattern.

In the same way as for the pattern 2, Figure 7.6(b) shows the displacement estimated by the sensor using only $I_{\mathrm{r}}$ and $I_{\mathrm{g}}$, and assuming $d_{0}$ was known. The RMS error for $d$ was $(75.9 \pm 44.2) \mu \mathrm{m}$, whereas the RMS error of $\hat{d}$ was $(6.2 \pm 4.6) \mu \mathrm{m}$. On average, resolving the displacement took $23.2 \mathrm{~ms}$ per measurement.

Figure 7.7 shows position estimations using Equation 7.5). In this case, also $I_{\mathrm{b}}$ is used to resolve the position without any knowledge of the previous position. Especially in the first $15 \mathrm{~mm}$, the sensor readout is wrong multiples of $l$, since $I_{\mathrm{b}}$ is not significantly changing and random variations of the signal cause the algorithm to find a minimum in the wrong period. Over approximately $25.1 \times 10^{3}$ measurement points, the estimation is $1,2,3$ or 4 times $l$ off in $6.1 \%$, $1.1 \%, 0.3 \%$ and $0.047 \%$ of the measurements, respectively. The RMS error of the position measurements is $(157.9 \pm 657.4) \mu \mathrm{m}$. Resolving the position using Equation 7.5 took $26.6 \mathrm{~ms}$ on average. 


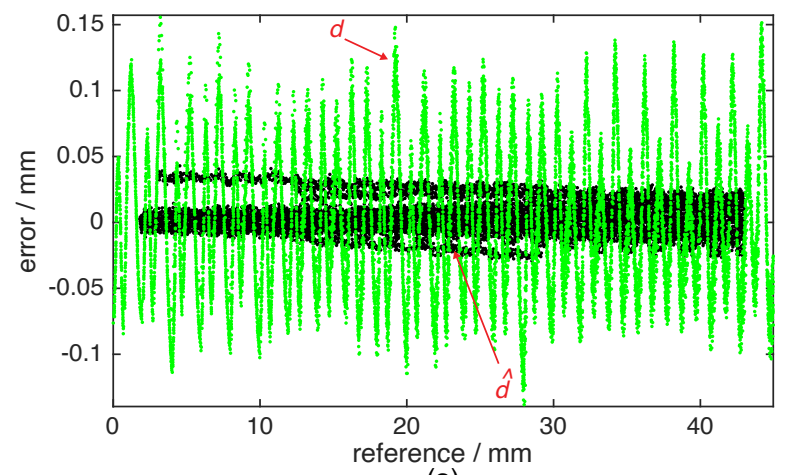

(a)

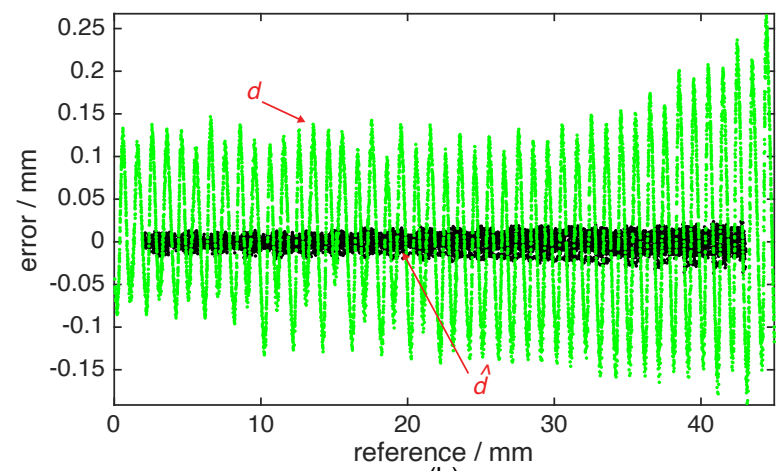

(b)

Figure 7.6: Distance measurements derived from $I_{\mathrm{r}}$ and $I_{\mathrm{g}}$ and a known $d_{0}$ using Equation 7.3 (d) and Equation 7.5 ( $\hat{d}$ ) based on (a) pattern 2 and (b) pattern 3.

\subsection{Discussion}

The presented MR safe RGB spectrophotometer-based single fiber position sensor proved to be able to repeatably measure the reflectance of a pattern moved in front of the fiber and use this to determine the direction and magnitude of displacement (two wavelengths) or the absolute position (three wavelengths). Especially the displacement measurements showed high accuracy with an RMS error down to $(6.2 \pm 4.6) \mathrm{\mu m}$, which is better than some commercially available MR safe and previously presented analog optical position sensors 200, 203. However, several aspects can be changed to improve future device iterations.

First of all, although we reach light intensity changes of over a million points per millimeter, the reported accuracy is only $(6.2 \pm 4.6) \mu \mathrm{m}$. Improving measurement setup may improve the reported accuracy since the current setup suffered from drift in the vibrometer or the resistance network connected to 


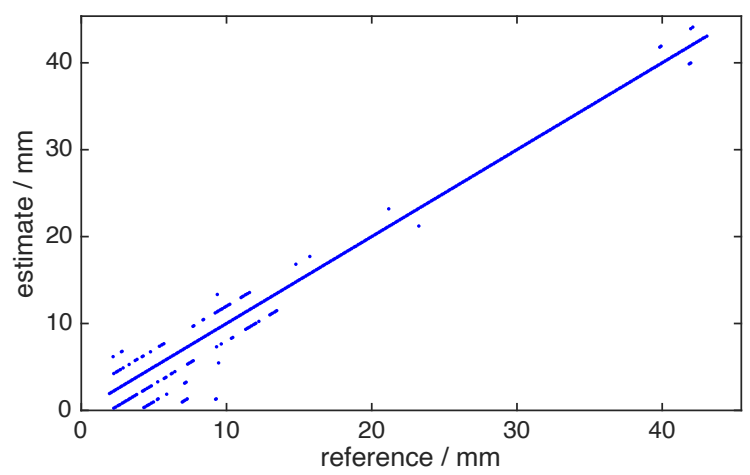

Figure 7.7: Position measurements based on pattern 3 obtained by solving Equation 7.5.

the vibrometer output. The measurements could be normalized using the end stops, but drift may still impact the measurements as each traversal was slow. Additionally, the vibrometer was aimed at a reflector mounted on top of the linear slider, which can amplify unwanted motions. An absolute position measurement as a reference would be preferred.

Furthermore, the optical setup may be improved. Light intensity fluctuations can occur due to light source instability, fiber bending or fiber mismanagement. A reference fiber could be used to compensate for transmission losses, fiber misalignments and fiber bending [206]. Specifically for our design, replacing the $2 \times 1$ optocoupler with a $2 \times 2$ optocoupler (e.g., IF-540, Industrial Fiber Optics, USA) would be enough to generate a reference signal. This configuration is also used by Peirs et al. [207. The reference signal can be subtracted from the measurement signal in the analog circuitry or after analog-to-digital conversion. This step would make the sensor more immune to short-term factors such a noise and long-term issues such as the wearing of the LED. By installing temperature compensated current sources (e.g., CL25, Supertex inc., USA), the light source can be more robust against temperature changes. Also, the driving current could be based on light output instead of driving the same current through each LED.

Finally, the simple models (Equation 7.1 and Equation (7.2) with which the patterns were implemented helped guide the design process. Still, more complex models are necessary to predict the signal output more accurately. The assumption that the patterns do not influence one another is not correct, as is visible in Figure 7.5. Each LED emits a normally distributed spectrum around the dominant wavelength. Especially the green and blue patterns show cross-talk, as the tails of the normal distributions of the green and blue LED are overlapping. Also, each color does not necessarily reflect the dominant wavelength of the corresponding LED. Also, while the implemented color gradient is linear, Figure 7.5.(c) shows that the reflectance shows a higher-order curve - apart from 
the cross-talk between the channels. This effect might be the gamma correction implemented in many consumer electronics to compensate for the non-linearity of the human eye. The effect can be compensated for in the design using a calibration curve, as done in 203. Lastly, the printer does not mix the colors as our models do; the printer approximates the desired colors with different inks, and, e.g., the color yellow will not be a mixture of green and red. Instead, the yellow toner is used, which has a different reflectance. We showed that we could partly compensate for these effects by using a Fourier series approximation of the calibration signal.

\subsection{Conclusion}

An MR safe linear position sensor was presented, which determines the position of the translator based on an RGB spectrophotometric measurement on a paper strip patterned with a printer. To show the added value of an RGB LED compared to a single color LED, reflectance measurements were performed on patterned strips modulating the reflection of either one, two or three wavelengths of the LED simultaneously.

The results show that each printed color reflects all three wavelengths, so the superposition of different modulation schemes leads to cross-talk of one channel to another. Furthermore, a linear intensity increase of a printed color does not lead to a linear intensity increase of the reflected light. The modulation of two wavelengths simultaneously with a $90^{\circ}$ phase shift (i.e., a quadrature modulation) allows to resolve the direction of displacement due to the phase difference. The magnitude of displacement could be determined with an RMS error down to $(7.6 \pm 7.2) \mathrm{\mu m}$. The pattern modulating three wavelengths could also measure displacement $((6.2 \pm 4.6) \mu \mathrm{m})$. In both modulation schemes, compensation for the higher harmonics of the measurement signal based on a previous characterization improved the RMS error six to twelve times. The superposition of three modulation schemes also showed promising results to measure the translator's absolute position. However, the RMS error of $(157.9 \pm 657.4) \mu \mathrm{m}$ is 25 times worse than the RMS error for displacement measurements.

This work shows that changing a monochromatic for a trichromatic LED allows adding more functionality to an optical channel, which reduces the number of optical channels needed for position sensing.

\subsection{Acknowledgements}

We would like to thank J. Oosterbeek for his advice on printing technologies and help with producing the samples. Additionally, we would like to thank our technicians, H. Kuipers, M.H. Schwirtz and S.M. Smits, for their advice on and help with putting together the setup. 


\section{General discussion}

The biopsy is a crucial step in diagnosing breast cancer, of which the success rate is heavily dependent on accurate lesion localization and needle placement. Especially when lesions are magnetic resonance imaging (MRI)-visible only, there are limitations in the accuracy, which can negatively impact the biopsy outcome.

This thesis aimed to develop robotic approaches to assist the radiologist with the biopsy procedure on magnetic resonance (MR)-detected lesions. A system was designed, which proved able to assist with preoperative data-to-patient registration, ultrasound acquisitions, deformation tracking, needle detection and needle placement. The following sections discuss the design of the setup, the implemented controllers, the ultrasound acquisitions and the accuracy of registration and needle placement in more detail. Furthermore, a future perspective is given on (steps towards) clinical trials and the role of ultrasound and MRI-guided robot-assisted interventions in the clinic.

\subsection{Robotic setup}

\subsubsection{Design}

Chapter 2 explains that to perform biopsies on MR-detected lesions outside the MRI-bore, the robot should be able to: perform patient-to-robot registration and preoperative MRI-to-patient registration; perform ultrasound acquisitions; assist with needle insertion. An end-effector was designed, which incorporates stereo cameras, lighting, a projector, a three-degrees-of-freedom actuated needle guide with a needle stop, an ultrasound probe and electronics to manage the functionalities and communicate with the robotic manipulator.

A vital point is the compactness and the high integration of all system components. The presented setup has the cameras integrated on the end-effector. In contrast, other systems for robotic ultrasound acquisition or ultrasoundguided needle insertion often have the camera setup separated from the robot 67, 72, 73, 97, 98. Furthermore, many designs have separated mechanisms for holding the needle and holding the ultrasound probe $74,97,99,101,128,131$. A benefit of a highly integrated system is that inter-system calibration and recalibration after displacing the system are not necessary. Additionally, by tilting the ultrasound probe around two axes with respect to the robotic flange, the probe could be mounted closer to the flange, and the robot could navigate closer to the bed and the patient. Although, for instance, Mathiassen et al. 178. tilted the probe around one axis, various other research aligned the probe with the flange $67,69-71,73,75,76,78,99,177,208,212$. The latter configuration 
increases the size of the end-effector since the cable extends above the probe, which is a disadvantage. Smaller probe types may take up even less space 74, 101]. Nevertheless, since robotically actuated ultrasound probes do not need an ergonomic shape, the next step could be to integrate the ultrasound array in the end-effector. Finally, by measuring the depth of the needle with a laser, the design of the needle stop takes little space and functions independently of the needle length. In other systems, the size of the needle stop is determined by the length of the needle [97]. A bonus of the laser-based position measurements is the sub-millimeter accurate insertion depth measurement. This accuracy is higher than strictly necessary during a biopsy procedure since the needle can extract $10-15 \mathrm{~mm}$ of tissue in the direction of insertion. Currently, a drawback of the system is that it needs a pneumatic connection to drive the needle brake. In future designs, the braking mechanism could be replaced by an electric equivalent, or audiovisual feedback could indicate the correct depth was reached.

One limitation in the current implementation is that the integrated projector is not used yet. As a result, deformable registration of the patient with respect to the preoperative MRI could only be based on marker detection. The necessity of marker detection and a preoperative MRI to determine the scanning trajectory also limits the system's flexibility; a robotic ultrasound-guided biopsy device that performs ultrasound-guided biopsies without the need for an MRI could also be used for other biopsy procedures.

With the current end-effector design, intraoperative surface reconstruction can be achieved using the projector and stereo cameras. However, a possible improvement may be replacing these with a camera with an integrated depth sensor (an RGB-D camera) such as the Realsense D435 (Intel Corporation, USA) or a lidar such as the L515 (Intel Corporation, USA). These cameras readily enable surface reconstructions based on depth measurements. These cameras may also be used for marker detection since they also appoint colors to each pixel in space.

\subsubsection{Controller}

Safety is an essential topic in medical robotics since the robots are in contact with patients and medical practitioners. Physical interaction is characterized by energy exchange, and thus, key features are the current potential and kinetic energy in the robot as well as the current power. In Chapter 3, an impedance controller was presented that avoids joint limits and restricts the energy released in the null space of the robot. Additionally, the robotic ultrasound acquisitions in Chapter 4 were performed with an impedance controller that limits the current power and energy of the robot by scaling the spring stiffness and the damping.

One advantage of impedance controllers is their clear and simple physical representation; the system can be seen as a spring and a damper connected between the end-effector and the desired position. This system results in the 
robot behaving naturally, even while interacting with its environment. The impedance controller reacts differently to collisions than, e.g., a position or velocity controller. For an impedance controller, the magnitude of applied force is a function of the spring constant and the distance between the end-effector and the desired position; a position or velocity controller may force itself to achieve the current desired position or velocity, leading to damage to both the robot and the environment.

Although impedance controllers have clear advantages regarding their response to current power and energy limits, this thesis has not studied the appropriate limits. The limits to power and energy in specific applications are a study in itself, and more can be read, e.g., in the work of Lachner et al. 213.

A disadvantage of the impedance controller is that the task space trajectory following is not that accurate, see, e.g., Chapter 3, Figure 3.6. Part of the inaccuracy comes down to imperfections in real-world scenarios, such as friction in the joints. This is reflected in, e.g., Figure 3.5(c), where the residual energy in the virtual spring is higher on the actual robot than in the simulations. Furthermore, in task space, the end-effector is not a point mass, and if this is not taken into account, the robot may not accurately follow a planned path. The task space behavior can be improved by modulating the task space damping and stiffness with the mass matrix of the robot, as was done in Chapter 5 . However, the passivity of the system may be compromised as the pseudo inverse of the Jacobian is used in this method. Generally, the accuracy of a position controller can be considered higher than the accuracy of an impedance controller. Therefore, for the biopsy phase, a position controller may be desirable. In Chapter 6, this issue was solved by moving the desired position until the ultrasound probe had the desired plane. In the biopsy phase, as long as the ultrasound plane is aligned with the biopsy target, the position-controlled needle guide is primarily accountable for the accuracy of the needle placement.

\subsubsection{Ultrasound acquisitions}

In the introduction of this thesis, we identified that robotic ultrasound breast volume scanners might improve acquisition quality, flexibility in terms of degrees of freedom and field of view and limit deformations caused by scanning.

Chapter 4 shows the incorporation of confidence map-based ultrasound feedback can improve the quality of robotic ultrasound scans. If the preplanned patient-specific trajectory had small misalignments relative to the phantom, the controller could correct this. Additionally, the work presented by Nikolaev et al. [120] shows that the quality of the obtained ultrasound volumes generated by this setup is comparable to regular B-mode ultrasound images.

Multiple scanning and biopsy experiments pointed out the system's flexibility. In Chapter 4 and 5 two very different phantoms were scanned with different trajectories. Additionally, although the scanning experiments were performed in 
prone position, the needle placement experiments performed in Chapter 2 and 6 show that the robot also functions in supine position. These experiments also show that the field of view is large and that in clinical practice, the device may also image auxilary lymph nodes. These lymph nodes are essential in breast cancer staging and cannot be imaged by ultrasound volume acquisition devices currently on the market.

The deformation imposed on the breast was around $5 \mathrm{~mm}$ for the breast volume acquisitions presented in Chapter 5 Although exact numbers are missing, this deformation appears to be smaller than the deformations imposed by conventional scanners that compress the breast and similar to the deformations due to buoyancy in, e.g., the work of Nikolaev et al. [65]. As a result, the images acquired with this setup are readily registered with preoperative MRI, as shown in Nikolaev et al. 120.

A strong point of the implemented ultrasound feedback is the algorithm's robustness for varying breast stiffnesses. The controller controls the contact area between the breast and the probe utilizing the mean confidence. On the other hand, ultrasound scanners that use a constant force to maintain acoustic coupling will cause varying deformations for different breast stiffnesses. However, an irregular curvature of the surface does influence the scanning results since a surface with a smaller radius of curvature should be deformed more to achieve the same contact area. Hence, the preoperative images could be used to minimize the impact of variations in curvature; the expected curvature can be measured, and the scanning trajectory can be planned with varying setpoints of the mean confidence. In sections where the radius of curvature of the breast is small, the confidence setpoint should be lower than in sections where the radius of curvature is large. Nevertheless, the controller in Chapter 4 does not have preoperative imaging at its disposal to adjust the confidence setpoint in real-time. This controller could reconstruct the surface based on a preliminary ultrasound scan before the actual ultrasound acquisition takes place.

\subsubsection{Accuracy}

Our robotic system can improve the accuracy of the registration of ultrasound with MRI and the accuracy of needle placement with respect to the conventional ultrasound- and MRI-guided biopsies. Additionally, it achieved a similar accuracy compared to existing robotic systems.

The registration accuracy reported in Chapter 2 is $1.74 \mathrm{~mm}$, whereas in the work presented by Nikolaev et al. 120 the roboticcally acquired images were registered with MRI images with an accuracy of $3.4 \mathrm{~mm}$. Fausto et al. 64 reported a manual registration error of $4.2-5.8 \mathrm{~mm}$.

The biopsy accuracy of our robotic setup is $3.03 \mathrm{~mm}$ and $2.89 \mathrm{~mm}$, as presented Chapter 2 and 6, respectively. These errors have the same order of magnitude, even while the biopsies in Chapter 6 had to take into account de- 
formations occurring in the phantom and the movement of the probe. The accuracy of the conventional ultrasound- and MRI-guided biopsies is approximately $10 \mathrm{~mm}$ and $5-6 \mathrm{~mm}$, respectively 40,46 . Previously presented robotic systems for breast biopsies reported accuracies in the range of 1.1 to $3.44 \mathrm{~mm}$ 83, 98.

It should be noted that inter-study comparisons are complex due to the different nature of the measurements. The results presented in this research are ex vivo with relatively rigid phantoms while Zhou et al. and Fausto et al. reported results obtained with actual patients. The results presented by El Khouli et al. were obtained ex vivo also. Although some of the reviewed robotic systems performed seemingly better than our system, due to many varying characteristics of the experiments, such as in-air versus phantom experiments and the number of registration steps involved, it is hard to compare them directly.

In the future, we could further improve the accuracy in several ways. Firstly, the smart servos of the three-joint serial kinematic chain of the needle guide use a gear transmission with some backlash. The backlash compromises mainly the precision of the procedure, as seen in the standard deviation of the results presented in Chapter 2, Figure 2.8 The impact of backlash can be minimized by repositioning the probe based on needle detection in the ultrasound images. Still, it is advisable to equip the end-effector with high accuracy backlash-less motors.

Furthermore, we could improve the system's calibration to improve the accuracy. There are multiple frames on the end-effector, which play an important role in the accuracy of the total system. These are the camera, needle guide, and ultrasound probe frames. We derived the needle guide and ultrasound probe frame from the computer-aided design (CAD) files of the end-effector. Accurate calibration of these frames is necessary to achieve higher accuracy. The needle guide frame can, for instance, be calibrated with the method described by Nelson et al. [102, where LEDs placed on both the end-effector and biopsy needle were tracked with a camera. The ultrasound probe's frame may be obtained in a similar fashion as presented by Ahmad et al. 214]. Both the ultrasound probe and a phantom were tracked optically in this work. The coordinate frame of the ultrasound probe could be estimated since the phantom contained some ultrasound-visible markers as well.

\subsection{Future perspective}

\subsubsection{Validation}

The added value of medical robots should be proven with evidence before they are adopted in clinical practice. This section discusses the current validation experiments and considerations about (the road to) in vivo testing. 


\section{Ex vivo}

The experimental validations presented in this work are based on phantoms with various degrees of complexity and realisticity. Most of the phantoms were based on a polyvinyl chloride plastisol (PVCP) mixture. These phantoms can be realistic regarding the shape and imaging characteristics. As was the case in Chapter 5 the shape of a phantom can be made identical to an actual breast by basing the phantom design on patient data. Furthermore, they can have varying signal intensity on ultrasound and MRI, and structures can be made selectively visible on either MRI or ultrasound. Additionally, different structures can be incorporated in one phantom with subsequent molding steps. However, the phantoms are much stiffer than real breasts. Although the stiffness of structures can be tuned with the ratio of plastisol to PVC, the material becomes more brittle as more plastisol is added, limiting the lowest achievable stiffness. As discussed in Chapter 6, the Young's modulus of the inner structures of the phantoms may be up to ten times stiffer than actual breast tissue 182 184. It is expected that this primarily influences the biopsy results, as the lesion would have a higher tendency to displace in actual breast tissue.

The quality of ex vivo ultrasound images may differ from imaging actual breast tissue. The quantity of ultrasound gel plays a minor role when PVCP is imaged than when human skin is imaged. Ultrasound gel is usually applied to prevent air from being between the probe and the skin. PVCP has some liquid trapped inside the material, making it less likely that air is between the probe and the material.

\section{Safety}

The manipulator and the end-effector have multiple attributes to ensure patient safety. The manipulator - the KUKA LBR MED 7 - has integrated safety features; next to the control cycle, the robot checks several boundary conditions and input signals at high rates. Both exceedances of boundary conditions and these input signals can activate the robot's brakes and multiple programmable signals. Force, velocity and location boundaries were implemented, the latter based on CAD drawings of the setup. The input signals are two emergency switches that could be triggered by hand or foot. Furthermore, as discussed in Chapter 2 , the end-effector's supply was connected to a programmable safety signal such that the motors of the needle guide are switched off in case of emergency as well.

However, there are some aspects regarding safety that need attention. In the case where custom controllers drive the manipulator via the research interface of the manipulator, it is advisable to make a software layer that checks the feasibility of all control inputs that are being sent to the robot. Even with the current safety measures in place, a programming error can send the robot off the desired trajectory several centimeters before being stopped. Furthermore, 
the end-effector in its current form cannot be disinfected. This issue could be addressed by separating the patient from the robot with an ultrasoundtransparent sheet.

\section{Considerations for clinical trials}

All suggestions regarding accuracy and safety discussed in the previous sections should be addressed to move on to clinical trials. Additionally, each subcomponent would need in vivo testing before testing the entire system.

The subcomponents that would need clinical validation are the ultrasound acquisitions, the ultrasound/MRI fusion, the deformation modeling and the deformation tracking. For ultrasound acquisitions, the approach for ethical approval and the experimental process could be very similar to the work presented on the automated cone-based breast ultrasound scanner [65]. In ultrasound/MRI fusion, rigid transformations can be validated utilizing the external skin markers as presented in de Jong et al. 119] or anatomical landmarks, as done in Nikolaev et al. 120], as the gold standards. The accuracy of deformable transformations may be validated by well-trained experts or external measurement devices such as an RGB-D camera 215]. Deformation modeling as presented in Groenhuis et al. 121 and deformation tracking as presented in Chapter 6 can be validated by placing a static reference ultrasound probe on the breast to determine the relation between the estimated and actual position of the tissue.

Finally, the biopsy should be validated. Usually, the accuracy of robotic MRIguided biopsies or ultrasound-guided biopsies on ultrasound-visible lesions can be determined by imaging the site with the needle in place $[103,216$. However, these methods are not suitable for the presented system because it is developed to perform ultrasound-guided biopsies on lesions that are not necessarily visible on ultrasound. The procedure could be applied to lesions visible on both ultrasound and MRI for validation purposes. Alternatively, a tissue marker could be placed during the procedure to confirm correct needle placement postoperatively with an additional MRI.

\subsubsection{Role in clinical practice}

Since both the ultrasound-guided and the MRI-guided robotic biopsies are focused on performing biopsies on MR-detected lesions, the question arises as to which system is preferable.

Currently, there are no guidelines on the accuracy of needle insertion in clinical practice, and in general, more accurate needle insertions result in more effective treatment or accurate diagnosis [104]. The MRI-guided robot has shown to be more accurate in phantom testing with an accuracy of $1.29 \mathrm{~mm}$ compared to $2.89 \mathrm{~mm}$ reported in this work 116 .

One advantage of ultrasound-guided biopsies is that the MRI-acquisition 
procedure remains unchanged other than that some markers should be attached to the patient's skin. Another advantage is that the system can be more widely applicable since it can also be applied when women would need a regular ultrasound-guided biopsy or an automated volumetric breast ultrasound, which may be the case for women with dense breasts. Additionally, the system could be applied in other diagnostic workups where ultrasound acquisitions and biopsies are commonplace, such as muscle disease diagnosis.

A disadvantage of the ultrasound-guided robotic biopsy is the dependence on a more extensive range of collaborating technologies and the prerequisite that each of these technologies individually should achieve maturity. These technologies include robot control, needle steering, registration, deformation modeling, ultrasound volume acquisitions and reconstruction. The robotic MRI-guided biopsies are primarily dependent on the development of actuation technology.

A disadvantage of the MRI-guided biopsy is the prolonged MRI time, making the procedure more expensive. The current procedure of MRI acquisitions should be changed, and the question is whether both the detection and the biopsy of the lesion can be performed in one go. Some discussion about the follow-up may be necessary, which in Dutch hospitals can take place during the multi-disciplinary consultation. In this case, the MRI time will be longer, and an additional contrast agent administration may be necessary. On the other hand, the robotic ultrasound-guided biopsies allow, in general, for more time between the MRI scan and the decision to intervene.

Overall, both systems show promising features, and it is too early to write off either option. The ultrasound-guided solution takes longer to fully mature due to all the technologies involved, but the efforts may pay off with lower MR time and greater flexibility. While other ultrasound-guided biopsies can be based on similar principles, this setup may even find its calling in a different clinical environment. 


\section{Conclusion}

This work aimed to develop robotic approaches to breast biopsies on lesions only visible with magnetic resonance imaging (MRI). Robots can function both outside or inside the MRI bore, but this work focused mainly on robotic assistance outside the MRI bore. A robotic setup was developed with an end-effector that enables a seven-degrees-of-freedom manipulator to assist in preoperative datato-patient registration, robot-to-patient registration, ultrasound acquisitions, deformation tracking, needle detection and needle placement. To this end, the end-effector contains an actuated needle guide, an ultrasound probe, a set of stereo cameras, a projector and lighting.

Multiple impedance controllers were implemented on the robot. The motion controller for the manipulator could limit the energy in the null space and avoid reaching the joint limits while respecting the current position of the end-effector.

Phantom experiments showed that the preoperative data-to-patient registration and the lesion targeting accuracy are higher than is currently the case in clinical practice. Registration was performed with an accuracy of $1.74 \mathrm{~mm}$ while in literature, the manual registration's accuracy was around $5 \mathrm{~mm}$. Needle placement was performed with an accuracy of $2.89 \mathrm{~mm}$. Literature states that the current accuracy for manual ultrasound-guided or MRI-guided biopsies is $10 \mathrm{~mm}$ and $5-6 \mathrm{~mm}$, respectively. The setup could compensate for deformations and needle positioning errors based on optical flow and needle detection algorithms. However, the system currently performs less accurately while depending on needle detection instead of the forward kinematics of the needle guide.

Multiple ultrasound acquisition algorithms were implemented with ultrasound feedback based on confidence maps. First, the ultrasound feedback only modified the translation and the rotation of the ultrasound probe in the image plane. The experiments show that the quality of robotic ultrasound scans improves if the patient-specific trajectory is supplemented with ultrasound feedback; the ultrasound feedback compensates for offsets or missing information in the reference trajectory with respect to the phantom. In follow-up work, the controller also modified the pose of the ultrasound probe out of the image plane. This modification shows that the ultrasound feedback can modify at least three degrees-of-freedom of the end-effector configuration. As such, the robot can achieve patient-specific trajectories based on more general input.

An magnetic resonance (MR) safe position sensor was developed based on a spectrophotometer for robots functioning inside the MRI bore. The experiments show that a single optical fiber gains functionality simply by replacing the singlecolor light-emitting diode by a multi-color one. The smaller form factor and the high accuracy make this sensor a valuable addition to future MR safe robots. 


\section{Summary}

In women, breast cancer is the most common cancer, and it is the leading cause of cancer death in many countries. In the diagnostic workup of breast cancer, the biopsy is crucial to determine the malignancy of a lesion. Its success rate mainly depends on accurate lesion localization and needle placement. Especially when lesions are magnetic resonance imaging (MRI)-visible only, there are limitations in the accuracy, which can negatively impact the biopsy outcome.

Robotic assistance can take place inside and outside the magnetic resonance (MR) bore and potentially improves the biopsy procedure's accuracy. For outside the MRI, the MRI and Ultrasound Robot-assisted biopsy (MURAB) project presents a robotic setup to assist the radiologist with an ultrasound-guided biopsy on an MR-detected lesion. The robotic setup consists of a seven-degreesof-freedom robotic arm holding an end-effector positioned under a patient bed. The patient lies on this bed with the examined breast through a hole such that it is freely accessible by the robot. The robot achieves an accurate notion of the lesion position by combining the preoperatively acquired MRI images with stereo vision and intraoperatively acquired ultrasound images. Additionally, a patient-specific biomechanical model is built utilizing elastography to predict deformations caused by needle insertion. For inside the MRI, an entirely plastic MR safe pneumatic robot was developed, which autonomously takes a biopsy based on the MRI images.

In this thesis, several aspects of these setups are worked out in detail. For the MURAB project, an end-effector design containing an actuated needle guide, an ultrasound probe, stereo cameras, a projector and lighting is presented. A compliant controller is introduced, limiting the robot's energy in the null space, optimizing the joint positions relative to their limits, and avoiding the joint limits. Furthermore, the work shows how ultrasound feedback is taken advantage of during ultrasound acquisitions and the biopsy procedure. For the MR safe robot, a position sensor is developed based on a spectrophotometer.

Several phantom experiments show that the robot more accurately registers the preoperatively acquired MRI images with the patient and more accurately targets the lesion than is currently the case in clinical practice. During robotic ultrasound acquisitions, ultrasound feedback in the form of confidence maps is utilized to correct a preoperatively planned trajectory, obtain higher quality ultrasound images, and fully autonomously scan a beforehand unknown surface. Additionally, confidence maps are used to determine when to start tracking the lesion position when approaching the patient, and needle detection is used to correct for errors in the current needle position. Overall, this thesis shows some of the potential benefits of introducing robotics to breast cancer diagnosis. 


\section{Samenvatting}

Borstkanker is de meest voorkomende kankervorm bij vrouwen en in veel landen de hoofdoorzaak van sterfgevallen door kanker. De biopsie is een cruciale stap in de diagnostiek waarmee bepaald wordt of een laesie kwaadaardig is. Het slagingspercentage van een biopsie hangt af van de accuraatheid van de plaatsbepaling van de laesie en het plaatsen van de naald. Vooral wanneer de laesie gedetecteerd is met magnetic resonance imaging (MRI), zijn er limitaties in deze accuraatheid die de uitkomst van de biopsie negatief kunnen beïnvloeden.

Robots kunnen zowel binnen als buiten de MRI assisteren met de biopsie en zodoende de accuraatheid verbeteren. Voor buiten de MRI presenteert het MRI and Ultrasound Robot-Assisted Biopsy (MURAB) project een robotische opstelling die de radioloog helpt een echogeleide biopt te nemen van een laesie die eerder middels MRI gedetecteerd is. De opstelling bestaat uit een robot met zeven vrijheidsgraden waaraan een eindeffector bevestigd is geplaatst onder een bed met daarop de patiënt. De te examineren borst hangt door een gat zodat deze vrij toegangkelijk is voor de robot. De robot krijgt een accuraat beeld van de huidige positie van de laesie door de eerder genomen MRI beelden te combineren met stereovisie en echobeelden. Verder wordt een patiëntspecifiek biomechanisch model opgebouwd aan de hand van elastografie, welke gebruikt kan worden om deformaties tijdens het plaatsen van de naald te voorspellen. Voor binnen de MRI is er een plastic, pneumatische, magnetic resonance (MR) safe robot ontwikkeld welke autonoom een MRI-geleide biopt kan nemen.

In deze thesis worden verschillende onderdelen van deze setups verder uitgewerkt. Voor het MURAB project wordt o.a. het ontwerp van de eindeffector gepresenteerd uitgerust met een geactueerde naaldgeleider, een echokop, stereocamera's, een beamer en verlichting. Verder wordt er een compliante besturing voor de robot geïntroduceerd welke de energie in de nulruimte van de robot limiteert, de posities van de assen optimaliseert ten opzichte van hun limieten en ook voorkomt dat de limieten geraakt worden. Tot slot wordt uitgewerkt hoe je echobeelden kunt gebruiken als terugkoppeling tijdens beeldopnames en het biopsieproces. Voor de MR safe robot is een positiesensor ontwikkeld gebaseerd op een spectrofotometer.

Middels fantoomexperimenten laten we zien dat de robot accurater preoperatieve MRI beelden kan registreren met de patiënt en de naald accurater kan plaatsen dan nu het geval is in de kliniek. Tijdens robotische echo-opnames kunnen confidence maps teruggekoppeld worden om het preoperatief geplande pad aan te passen en een betere kwaliteit echobeelden te verkrijgen. Ook onbekende oppervlakken kunnen autonoom gescand worden. Verder zijn confidence maps ingezet om te bepalen wanneer het systeem kan starten met het volgen van de laesie, en naalddetectie wordt ingezet om fouten te corrigeren in de naaldplaatsing. Al met al laat deze thesis de mogelijke voordelen zien die het inzetten van robotica bij de diagnose van borstkanker kan bieden. 


\section{List of publications}

\section{In preparation}

M. K. Welleweerd, T. Hageman, M. Pichel, D. Van As, H. Keizer, J. Hendrix, R. Kräwinkel, A. Mir, N. Korkmaz, and L. Abelmann, "Performance and application of a simple automated Magnetic Optical Density meter for analysis of Magnetotactic Bacteria," pp. 1-23, Jun. 2021. arXiv: 2106.07466

M. K. Welleweerd, L. Abelmann, S. Stramigioli, and F. J. Siepel, "MR Safe RGB Spectrophotometer-based Single Fiber Position Sensor," In preparation,

M. K. Welleweerd, S. S. Groothuis, S. Stramigioli, and F. J. Siepel, "Combining Geometric Workspace Compliance with Energy-based Joint Limit Avoidance," In preparation,

\section{Journals}

F. J. Siepel, B. Maris, M. K. Welleweerd, V. Groenhuis, P. Fiorini, and S. Stramigioli, "Needle and Biopsy Robots: a Review," Current Robotics Reports, vol. 2, no. 1, pp. 73-84, Mar. 2021. DOI: 10.1007/s43154-020-00042-1

V. Groenhuis, A. Nikolaev, S. H. G. Nies, M. K. Welleweerd, L. de Jong, H. H. G. Hansen, F. J. Siepel, C. L. de Korte, and S. Stramigioli, "3-D Ultrasound Elastography Reconstruction Using Acoustically Transparent Pressure Sensor on Robotic Arm," IEEE Transactions on Medical Robotics and Bionics, vol. 3, no. 1, pp. 265-268, Feb. 2021. DOI: 10.1109/TMRB.2020.3042982

M. K. Welleweerd, F. J. Siepel, V. Groenhuis, J. Veltman, and S. Stramigioli, "Design of an end-effector for robot-assisted ultrasound-guided breast biopsies," International Journal of Computer Assisted Radiology and Surgery, vol. 15, no. 4, pp. 681-690, Apr. 2020. DOI: 10.1007/s11548-020-02122-1

L. de Jong, M. K. Welleweerd, J. C. van Zelst, F. J. Siepel, S. Stramigioli, R. M. Mann, C. L. de Korte, and J. J. Fütterer, "Production and clinical evaluation of breast lesion skin markers for automated three-dimensional ultrasonography of the breast: a pilot study," European Radiology, vol. 30, no. 6, pp. 3356-3362, Jun. 2020. DOI: $10.1007 / \mathrm{s} 00330-020-06695-y$

A. Dijkshoorn, P. Werkman, M. Welleweerd, G. Wolterink, B. Eijking, J. Delamare, R. Sanders, and G. J. M. Krijnen, "Embedded sensing: integrating sensors in 3-D printed structures," Journal of Sensors and Sensor Systems, vol. 7, no. 1, pp. 169-181, Mar. 2018. DOI: 10.5194/jsss-7-169-2018

M. Rafeie, M. Welleweerd, A. Hassanzadeh-Barforoushi, M. Asadnia, W. Olthuis, and M. Ebrahimi Warkiani, "An easily fabricated three-dimensional threaded lemniscate-shaped micromixer for a wide range of flow rates," Biomicrofluidics, vol. 11, no. 1, p. 014 108, Jan. 2017. DOI: 10.1063/1.4974904 


\section{Conference proceedings}

M. Lagomarsino, V. Groenhuis, M. Casadio, M. K. Welleweerd, F. J. Siepel, and S. Stramigioli, "Image-guided Breast Biopsy of MRI-visible Lesions with a Handmounted Motorised Needle Steering Tool," in 2021 International Symposium on Medical Robotics (ISMR), 2021 (accepted)

M. K. Welleweerd, A. G. de Groot, V. Groenhuis, F. J. Siepel, and S. Stramigioli, "Out-of-Plane Corrections for Autonomous Robotic Breast Ultrasound Acquisitions," in 2021 IEEE International Conference on Robotics and Automation (ICRA), 2021, pp. 12 515-12521, ISBN: 9781728190778. DOI: 10.1109/ ICRA48506.2021.9560865

M. K. Welleweerd, D. Pantelis, A. G. de Groot, F. J. Siepel, and S. Stramigioli, "Robot-assisted ultrasound-guided biopsy on MR-detected breast lesions," in 2020 IEEE/RSJ International Conference on Intelligent Robots and Systems (IROS), IEEE, Oct. 2020, pp. 2965-2971, ISBN: 978-1-7281-6212-6. DOI: 10 1109/IROS45743.2020.9341695

M. K. Welleweerd, A. G. De Groot, S. O. H. De Looijer, F. J. Siepel, and S. Stramigioli, "Automated robotic breast ultrasound acquisition using ultrasound feedback," in International conference of Robotics and Automation, 2020, pp. 9946-9952, ISBN: 9781728173955

V. Groenhuis, E. Tagliabue, M. K. Welleweerd, F. J. Siepel, J. D. Munoz Osorio, B. M. Maris, D. Dall'Alba, U. Zimmermann, P. Fiorini, and S. Stramigioli, "Deformation Compensation in Robotically-Assisted Breast Biopsy," in 11th International Conference on Information Processing in Computer-Assisted Interventions, Jun. 2020

A. V. Nikolaev, L. de Jong, V. Groenhuis, M. K. Welleweerd, F. J. Siepel, S. Stramigioli, H. H. G. Hansen, and C. L. de Korte, "Quantitative Evaluation of Automated Robot-Assisted Volumetric Breast Ultrasound," in 2020 IEEE International Ultrasonics Symposium (IUS), IEEE, Sep. 2020, pp. 1-4, ISBN: 978-1-7281-5448-0. DOI: 10.1109/IUS46767.2020.9251310

L. de Jong, M. K. Welleweerd, J. C. van Zelst, F. J. Siepel, S. Stramigioli, J. J. Fütterer, R. M. Mann, and C. L. de Korte, "Breast lesion markers for 3D ultrasound examinations of the breast," in European Congress of Radiology, Vienna, 2018, pp. C-2956. DOI: 10.1594/ecr2018/C-2956

V. Groenhuis, F. J. Siepel, M. K. Welleweerd, J. Veltman, and S. Stramigioli, "Sunram 5: An MR Safe Robotic System for Breast Biopsy," in The Hamlyn Symposium, 2018, pp. 85-86. DOI: 10.31256/hsmr2018.43

A. Dijkshoorn, P. Werkman, M. Welleweerd, G. Wolterink, J. Delamare, R. Sanders, and G. J. M. Krijnen, "Embedded sensing: making the best of 
3D printed sensors," in Proceedings Sensor 2017, AMA Service GmbH, VonMünchhausen-Str. 49, 31515 Wunstorf, Germany, 2017, pp. 431-437. DOI: 10.5162/sensor2017/D1.3 


\section{Acknowledgements}

As far as I am concerned, I have now come to the most-read section of every thesis, while my motivation to type yet another word has reached an all-time low. However, this thesis would not exist in its current form, and I would never be the person I currently am without the help of quite some people. So I will give it a go.

Stefano, I would like to thank you for giving me the opportunity to pursue my $\mathrm{PhD}$ at your chair. These four years were packed with great experiences that will be valuable for the rest of my career and life.

Françoise, the first time we met, I thought we would casually talk about the contents of the $\mathrm{PhD}$ position. In reality, it turned out to be a job interview with a full-blown application committee including Stefano and Vincent. I remember the shock when Vincent asked 'what makes you think you are a suitable candidate?,' but I am happy that my answer was satisfactory and I got the offer. We had the craziest adventures in these four years while we dragged the robot through Europe, leading us past the Northern Lights, one-way streets in Brussels and extremely narrow parking spots in London. I appreciate all the (mental) support I got during these years, which was definitely needed at times.

Vincent, thanks for these four years in which we worked together on many different topics. Who knew air could become the main ingredient of a robot? Please keep me updated about all the inventions you will do in the coming years.

I would like to thank all of the partners in the MURAB project for their excellent collaboration and their generous hospitality when we met at each institution. I really enjoyed the meetings where we met at each institution the online ones were less great. In particular, I would like to thank the guys of KUKA for their warm welcome at their facilities in Augsburg, for teaching me all the ins and outs of their robots, and for endlessly texting back and forth about things like the mass matrix of the robot.

Leon, we met during my bachelor studies right after I found this interesting description of an assignment titled 'The bucket brain.' After that, we kept working together on different projects in different locations, which I really enjoyed. Although I think you still cannot wake me up during the night to ask me about my research questions, you have taught me a great deal about becoming an independent researcher. Thanks for that.

Gijs, you enthused me about doing a master thesis about 3D printing. That, and the moment you suggested me as a possible $\mathrm{PhD}$ candidate to Stefano and Françoise were the first steps toward the creation of this thesis. I think you chairing my defense really completes it. Thanks.

Rob, you arrived at the university at exactly the same time as me, and I am glad you did. You are always the life of the party; you are the quizmaster, the person that reserves the tables at the Gallery, that brings beers, or that carries around the Game Cube including controllers. Thank you for all the times that 
you and Laura invited me for dinner, drinks, games and sports, and for the fun trips we made to Emmen and Texel. Of course, we will continue doing these things, and I am already looking forward to them.

Toon, we got to know each other quite well during your internship, your Master's assignment, and later, while being colleagues. It was always a pleasure to work together. Without your coding skills and the support you gave students after you, the robotic setup would never have functioned like it is currently doing. Aside from being a great colleague, I got to know you as a friend with whom I could discuss anything during the ride home or while enjoying a beer.

Stefan, we had fruitful cooperations both in and outside of work. Surely, somewhere in the future, we will come up with something brilliant again. Also, I would like to promote your app here, hiittoget.fit, which ensured my heart could handle all the $\mathrm{PhD}$ stress.

Johan, I truly found it a pity when you left RAM and Enschede since we always had the greatest extended vrimibo's, Mario Kart sessions and Comedy nights. However, every cloud has a silver lining: we had to finish your beer voucher at Proeflokaal Belgie. This will remain a great memory, even though thinking about it gives me a small headache again.

I would like to thank all the (former) colleagues of the 'Best office', but of course also the 'Golden boys' and others for the good atmosphere, countless lunches, coffees, vrimibo's, weird conversation topics, comedy nights, dinners, games, memes, you name it. Elfi, Frieda, Gerjan, Hengameh, Jornt, Maaike, Marieke, Martijn, Mourad, Alexander, Riccardo, Luuk, Astrid, Christophe, Dimitris, you made sure I always enjoyed going to work. Furthermore, I would like to thank all the people that participated in the RAM futsal team. The futsal match was always one of the highlights of my week. I hope the RAM team will continue to be a hit, and if you ever need stand-ins, please let me know.

Gerben, Henny, Marcel, Sander, I would like to thank you for all the technical know-how, support, and all the jokes, even though some of them I heard at least a dozen times - Pardon?! Yes, paarden!

I would like to thank all the students that I had the pleasure of working with during my PhD. I learned a lot in the process, and I hope so did you. In particular, I would like to thank Stijn and Jim, whose work is included in two of the chapters of this thesis.

Jolanda, thank you for chasing Stefano every time Hora Finita needed more input, for always washing the shirts of the futsal (i still think the team should do it themselves), organizing team activities, but above all for the nice conversations we had when I poked my head around the corner of your office.

Jeroen, we got to know each other so well during our studies and while being house mates, that just like this (?), two words are enough to refer to a certain joke or moment. Making balloon animals, talking about socks, getting heavily injured while eating a salad; all manner of things happened. Together with Mathijs, we gamed our way through lock downs. If we got a cent for every hour 
we played Duck Game together, we would not be rich, but we could buy anything of around ninety cents. Mathijs, sometimes your humor goes so high over my head, that I only understand it after minutes. I hope to enjoy this and all of our sparring sessions about weird projects like ceiling-crawling robots, LED-lit house numbers and autonomous lawnmowers for much longer.

I would like to thank the COVID survivors. Damir and Edita, you always ensured that some much-needed holidays were implemented in my busy PhD schedule. The house in Raẑanac and Damir behind a steering wheel were a guarantee for adventure. Google Maps was not needed to find dolphins, scorpions and war relics. Rosana and Adi, I can certainly recommend trying out the 'AH verspakket' some time, even though you are managing the process of picking ingredients currently available in your fridge and closet quite well. Thank you for all the times we were warmly welcomed at the Meteorenstraat.

Last but not least, wil ik graag mijn familie bedanken. Pap, mam, en natuurlijk ook Frank en Ester, en Ruben. Bedankt dat jullie me altijd gesteund hebben. Gedurende mijn studie, waar ik o.a. een mooie stage in Australië mocht doen, en gedurende mijn $\mathrm{PhD}$ traject. Ook al woon ik momenteel in Enschede, mijn tripjes naar Ommen voelen nog altijd als thuiskomen. Er is daar altijd iemand die luistert naar nieuwe ontwikkelingen of frustraties, en het vertrouwen dat ik het ging halen was altijd groot. Ik twijfel er niet aan dat dat zo blijft, welke kant ik nu ook op ga. Bedankt daarvoor.

That's it!

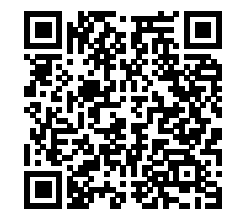


Appendices 



\section{A Performance and application of a simple automated Magnetic Optical Density meter for analysis of Magnetotactic Bacteria}

\section{Adapted from:}

M. K. Welleweerd, T. Hageman, M. Pichel, D. Van As, H. Keizer, J. Hendrix, R. Kräwinkel, A. Mir, N. Korkmaz, and L. Abelmann, "Performance and application of a simple automated Magnetic Optical Density meter for analysis of Magneto-tactic Bacteria," pp. 1-23, Jun. 2021. arXiv: 2106.07466

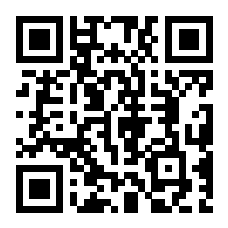




\begin{abstract}
We present a spectrophotometer (optical density meter) combined with electromagnets, dedicated to the analysis of magneto-tactic bacteria. We ensured that the system can be easily reproduced by keeping the complexity and price of the system low, and by providing the source of the 3D prints for the housing, the electronic designs, circuit board layouts, and microcontroller software. We compare the performance of this novel system to existing adapted commercial spectrophotometers. We demonstrate its use by analyzing the absorbance of magneto-tactic bacteria as a function of their orientation relative to the light path and their speed of reorientation after rotating the field by $90^{\circ}$. We continuously monitored the development of a culture of magnetotactic bacteria for five days and measured the development of their velocity distribution for an hour. Even though this dedicated optical density meter is relatively simple and inexpensive, the data extracted from suspensions of magneto-tactic bacteria is rich in information and will help the magneto-tactic research community understand and apply this intriguing micro-organism.

Note - This work is very much a team effort, which is reflected in the number of co-authors on this paper. My role was as follows: I designed the last versions of the mainboard of the optical density meter (1.1, 2.0 and 2.1) and multiple iterations of the measurement electronics. I assembled and tested parts of the electronics. I advised and supervised students that took part in this project. Furthermore, I contributed to the initialization and realization of this paper. My contributions were mainly focused on the layout, editing, and the design section.
\end{abstract}




\section{A.1 Introduction}

Magnetotactic bacteria posses a chain of iron-oxide or iron-sulfide nanocrystals that makes them align with the earth magnetic field [217, 218]. This property allows them to search efficiently for the optimimum redox conditions in stratified water colums 219]. Schüler and colleagues discovered that the transmission of light through suspensions of magnetotactic bacteria is influenced by the direction of an externally applied field [220]. This effect has been succesfully applied as a simple method to monitor for instance the cultivation of magnetotactic bacteria 221 224], and to assess their velocity 225, 226].

\section{A.1.1 Research question and relevance}

Commonly, the field-dependent transmission of light through a suspension of magnetotactic bacteria is measured by expanding a standard spectrophotometer with a magnetic add-on. These spectrophotometers are also known as optical density meters, and are commonly used in biolabs to determine cell concentrations.

The modification of existing spectrophotometers with magnetic add-ons has several disadvantages: these instruments are relatively complex and expensive, so modifications are mostly done on depreciated equipment; most instruments contain magnetic components that disturb the field and there is generally little space to mount electromagnets, certainly not in three dimensions; the various types of spectrophotometers and magnetic field generators and the variations between laboratories lead to a lack of a standardized measurement; more fundamentally, most spectrometers are not intended for sub-second continuous registration of absorbance over time. They are operated manually, and often use flash lights.

In this publication we present a spectrophotometer that intimately integrates the optical components with a magnetic field system, and is dedicated to the research on magnetotactic bacteria (figure A.1. Additionally, the design considers that students at the master or early PhD level should be capable to construct such an instrument, both with respect to complexity and price. Our main research question was how this new magnetic optical density meter (MagOD) compares to existing adapted spectrophotometers, and which novel measurement strategies it enables.

\section{A.1.2 Previous work}

The system we want to construct is still a spectrophotometer, but than combined with a magnetic field system. It is therefore useful to compare with commericial spectrophotometers. These systems generally use a Xenon light source and monochromator with a large wavelength range. Table A.1 lists an overview of specifications of representative commercial systems (Biochrome Ultrospecs 


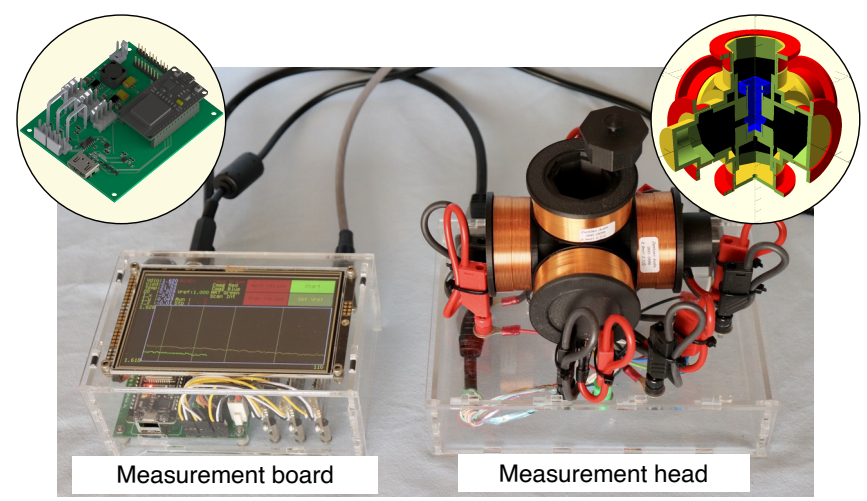

Figure A.1: Photograph of an open-source spectrophotometer with magnetic field option (MagOD). The system consist of a measurement head (left) in which a cuvette with a suspension of magnetotactic bacteria is inserted. The measurement board (right) is dedicated to control of the magnetic field, data acquisition and communication with the user over a touchscreen and wifi. The design of the system is open, including layout for electronic circuit boards (top left), 3D print source files (top right) and control software.

and the Eppendorf Biophotometer used by us for comparison), including their wavelength range $\left(\lambda_{\min }-\lambda_{\max }\right)$, spectral bandwidth $\left(\Delta_{\lambda}\right)$, maximum absorbance $\left(O_{\mathrm{D}}\right.$, see equation A.2 and approximate price.

The first spectrophotometer modified with a magnetic field module was presented by Schüler [220]. This device was based on standard optical components and used a permanent magnet generating a $70 \mathrm{mT}$ field. Later versions were constructed around commercial optical density meters such as the ones presented by Lefèvre 225. (based on a Varian Cary 50 UV) and Song [224] (based on a Hitachi U2800). In their case, the magnetic field is generated by coil systems that can generate adjustable fields up to $6 \mathrm{mT}$ [225].

Also, table A.1 presents the MagOD system we introduce in this paper. Its optical properties and price range compare well to standard commericial systems, whereas its field range is similar to the adapted systems by Lefèvre and Song.

\section{A.1.3 Structure and contents}

In this paper, we first discuss a model on the relationship between the transmission of light and the orientation of magnetotactic bacteria (Section A.2). Next to the specifications listed in table A.1, we defined other specifications that are important for the analysis of magnetotactic bacteria and the open source nature of the instrument. Our design choices are discussed in section A.3. The results section is divided into two parts. In section A.4.1. we analyse the performance of our current implementation and compare it to a commerical optical density meter. Section A.4.2 illustrates the possibilities of the novel system by giving 
Table A.1: Optical density meters.

\begin{tabular}{lrrrrrr}
\hline & $\begin{array}{r}\lambda_{\min } \\
(\mathrm{nm})\end{array}$ & $\begin{array}{r}\lambda_{\max } \\
(\mathrm{nm})\end{array}$ & $\begin{array}{c}\Delta_{\lambda} \\
(\mathrm{nm})\end{array}$ & $O_{\mathrm{D}}$ & $\begin{array}{c}B \\
(\mathrm{mT})\end{array}$ & $\begin{array}{r}\text { Price } \\
(\mathrm{Eu})\end{array}$ \\
\cline { 2 - 7 } & 190 & 1100 & 0.5 & 8 & & 12000 \\
Ultrospec 8000 & 230 & 600 & 4 & 3 & & 5000 \\
Biophotometer D30 & 600 & 600 & 40 & 2.3 & & 1300 \\
Ultrospec 10 & 637 & 637 & 18 & & 70 & \\
Schüler 220], 1995 & 190 & 1100 & 1.5 & 3.3 & $0-6$ & \\
Lefèvre [225], 2009 & 190 & 1100 & 1.5 & 6 & $0-4.3$ & \\
Song 224], 2014 & 465 & 640 & 25 & 2 & $0-5$ & 2000 \\
MagOD (this study) & & & & & &
\end{tabular}

four examples of experiments to extract information on the magnetic behaviour of magnetotactic bacteria. This instrument is still very much work in progress, and we invite the magnetotactic bacteria community to participate. For this, we indicate possiblities for improvements and ideas for further applications in section A.5

\section{A.2 Theory}

The standard method to determine the fraction of bacteria with magnetosomes in a culture is to observe the changes of light transmitted through a suspension of bacteria under rotation of a magnetic field [220]. The transmission of light is dependent on the relative orientation of the bacteria to the light path. For MSR-1, which are long, slender bacteria, the transmission is high when the field is perpendicular to the light path, whereas it is low when the field is aligned parallel to the light path. This is somewhat counter-intuitive, since MSR-1 have the smallest projected cross-section when they are aligned along the line of view. (As an analogue to blinds, MSR-1 let the light pass if the blinds are closed).

It is important to realize that we measure the intensity of light reaching the photodetector. The light leaving the light source can either be absorbed by the suspension of bacteria, or be scattered sideways so that it does not reach the photodetector. Highly dense suspensions of magnetotactic bacteria have a white appearance like milk. In analogy to milk, it is therefore very likely magnetotactic bacteria scatter, rather than absorb, light. MSR-1 are small compared to the wavelength of the incident light, especially considering their cross-section. Additionally, their index of refraction is only slightly higher than the surrounding liquid. These small 'optically soft' objects scatter more light in the forward direction if their projected area along the light path increases [227]. This would explain why the light intensity on the photodetector drops if the 


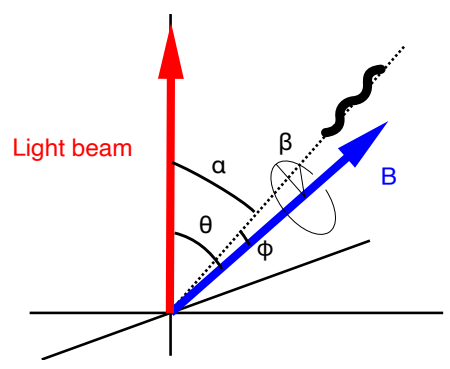

Figure A.2: Defintions of various angles. In the MagOD we set the angle $\theta$ between the light path and the magnetic field $B$. The bacteria align into the direction of the field, but can deviate by a small angle $\phi$, in a cone around the field direction described by $\beta$. As a result, the angle between the bacteria long axis and the light is $\alpha$. In case of sufficiently large fields, $\phi=0, \alpha=\theta$ and $\beta$ is irrelevant.

MSR-1 are aligned with the light beam.

For MSR-1, the projected area is roughly proportional to the sine of the angle between the long axis of the bacteria body and the light path. Due to Brownian motion and flagellar movement, the bacteria will not be aligned perfectly along the field direction but show an angular distribution. The width of this distribution will reduce with increasing field. In the following, we develop a simple theory to account for this effect. Since the MagOD meter allows us to accurately adjust the angle and strength of the magnetic field, we can use it to validate the approximation.

\section{A.2.1 Angle dependent scattering, $C_{\text {mag }}$}

We define the angle between the light path and the MSR-1 long axis as $\alpha$ (see figure A.2 and introduce a scattering factor relative to the intensity of light reaching the photodetector $(I(\alpha)$ with unit $\mathrm{V})$.

$$
g(\theta)=\frac{I_{\max }-I(\alpha)}{I_{\max }-I_{\min }}
$$

For MSR-1 the photodetector signal $I$ has a maximum when the MSR-1 are aligned perpendicular to the light beam $\left(I_{\max }=I(90)=I_{\perp}\right)$, at which point scattering, $g(90)$, is minimal.

Schüler [220] introduced a parameter to characterize the relative fraction of magnetotactic bacteria by comparing the light reaching the detector for the magnetic field aligned parallel and perpendicular to the light path $\left(C_{\mathrm{mag}}\right.$, 'coefficient of magnetically induced differential light scattering' or 'ratio of scattering intensities' 228]). Assuming that the scattering intensity can be estimated from the reduction of light reaching the detector as compared to the reference value of a sample without bacteria $\left(I_{\text {ref }}\right)$, the original definition is 


$$
C_{\mathrm{mag}}^{*}=\frac{I_{\mathrm{ref}}-I(0)}{I_{\mathrm{ref}}-I(90)} .
$$

With increasing concentration of bacteria, the total amount of light reaching the photodetector will decrease. In microbiology, traditionally cultures are characterized by a parameter ('optical density') that relates the reduction in light intensity to the reference value on a 10 -base log scall ${ }^{1}$

$$
O_{\mathrm{D}}(\alpha)=\log \left(\frac{I_{\text {ref }}}{I(\alpha)}\right)=\log \left(I_{\text {ref }}\right)-\log (I(\alpha))
$$

After the pioneering work of Schüler, researchers started to equip these optical density meters with magnetic fields. Using these instruments, it is more convenient to define $C_{\text {mag }}$ as $224,228,230,231$

$$
C_{\mathrm{mag}}=\frac{O_{\mathrm{D} \|}}{O_{\mathrm{D} \perp}}=\frac{\log \left(I_{\mathrm{ref}}\right)-\log (I(0))}{\log \left(I_{\mathrm{ref}}\right)-\log (I(90))}
$$

Nowadays, the latter definition is commonly used. It should be noted however that the values are not identical, not even for $C_{\text {mag }}$ close to unity (see appendix A.7). Since in the absence of magnetotactic bacteria $C_{\text {mag }}$ equals unity, often $\left(C_{\mathrm{mag}^{-}}-1\right)$ is plotted $223,228,231,233$.

Next to the ratio, it is insightful to study the absolute difference between the absorbances in the parallel and perpendicular direction

$$
\begin{aligned}
\Delta_{\mathrm{OD}} & =O_{\mathrm{D} \|}-O_{\mathrm{D} \perp} \\
& =\log (I(90))-\log (I(0))
\end{aligned}
$$

This difference is proportional to the absolute amount of magnetotactic bacteria that rotate in the field.

\section{A.2.2 Dynamic response}

When measuring $C_{\text {mag }}$ with adapted photospectrometers, the $O_{\mathrm{D}}$ values are measured over a long interval and the actual rotation of the bacteria is not measured. The MagOD system however can measure at sub-second intervals and monitor the dynamic behaviour of the bacteria. The response of bacteria to a change in field direction is determined by the balance between magnetic torque and rotational drag torque [234 236]. Alignment of a bacterium to an external magnetic field with angle $\overline{\phi(t)}$ (see figure A.2 can be described by a simple differential equation:

\footnotetext{
${ }^{1}$ Analogue to the Beer-Lambert law. It should be noted however that the relation between $O_{\mathrm{D}}$ and cell concentration is only approximate 229 .
} 


$$
f \frac{\partial \phi(t)}{\partial t}+m B \sin \phi(t)=0
$$

where $f[\mathrm{Nms}]$ represents the rotational drag coefficient, $m\left[\mathrm{~A} \mathrm{~m}^{2}\right]$ the magnetic dipole moment of the bacterium, and $B[\mathrm{~T}]$ the magnetic field strength.

For the determination of $C_{\mathrm{mag}}$, we rotate the field by $90^{\circ}$ very quickly. Therefore, initially we can assume the bacterium to be orthogonal to the magnetic field $\phi(0)=\pi / 2$. Solving the differential equation than leads to:

$$
\begin{aligned}
\phi(t) & =2 \cot ^{-1} \exp \left(\frac{m B}{f} t\right) \\
& \approx \frac{\pi}{2} \exp \left(-0.85 \frac{m B}{f} t\right) .
\end{aligned}
$$

The approximation is better than $0.065 \mathrm{rad}$ (appendix A.8). The angle $\phi$ can be indirectly estimated from the measured scattering as described by equation A.1. if we assume that the bacteria remain in the plane of rotation $(\beta=0)$. The settling time of this transition period is characterised by time constant $\tau=f / m B$. As in Pichel et al. [234, we scale the response time to the magnetic field, introducing a general rotational velocity parameter $\gamma(\mathrm{rad} / \mathrm{Ts})$ :

$$
\gamma=\frac{m}{\pi f}=\frac{1}{\pi \tau B} .
$$

\section{A.2.3 Brownian Motion}

When we remove the magnetic field, magnetotactic bacteria will quickly reorient in a random orientation distribution by Brownian motion, and possibly their flagellar motion. For the same reason, the bacteria will not align perfectly along the magnetic field. The alignment will become better at higher fields, so we may expect $C_{\text {mag }}$ to be field dependent. We consider the effect of Brownian motion first.

The propability distribution of finding MTB tilted at an angle $\phi_{0}$ from the magnetic field direction, $b\left(\phi_{0}\right)$, is determined by the ratio of magnetic $(-m B \cos (\phi))$ and thermal energy $(k T)$ according to the Boltzmann distribution (see e.g. textbook by Kittel, chapter 12 [237]). We should take into account that energy states for a specific value of $\phi$ exist in a full revolution around the field axis $(\beta=0 . .2 \pi)$. Therefore 


$$
\begin{aligned}
b\left(\phi_{0}\right) & =\frac{\int_{0}^{2 \pi} e^{a \cos \phi_{0}} \sin \left(\phi_{0}\right) d \beta}{\int_{0}^{\pi} \int_{0}^{2 \pi} e^{a \cos \phi} d \beta d \phi} \\
& =\frac{a}{2 \sinh (a)} \sin \left(\phi_{0}\right) e^{a \cos \left(\phi_{0}\right)},
\end{aligned}
$$

where $a=m B / k T$, with $k\left(\mathrm{~J} \mathrm{~K}^{-1}\right)$ the Boltzmann constant and and $T(\mathrm{~K})$ the temperature.

As a first order approximation, we assume that the scatter factor is proportional to projection of the bacteria shape on the light direction. Defining $\alpha$ as the angle between the bacteria long axis and the lightpath, the scattering factor (equation A.1) than becomes

$$
g(\alpha)=1-|\sin (\alpha)|
$$

The angle $\alpha$ is the combined result of the angle between the light and the field direction $\theta$ and the angle between the bacteria and the field $\phi$. One can show that the relation between $\alpha$ and these three angles is

$$
\cos (\alpha)=-\sin (\theta) \sin (\phi) \cos (\beta)+\cos (\theta) \cos (\phi)
$$

resulting in an expression for the scattering factor

$$
g(\theta, \phi, \beta)=1-\sqrt{1-\cos (\alpha)^{2}}
$$

The average scattering factor can be obtained by double numerical integration, first over all values of $\beta$ and than over the distribution of $\phi$

$$
<g(\theta)>=\int_{0}^{\pi} g(\theta, \phi) b(\phi) d \phi
$$

The numerical integration was performed in python, the source code of which is available as Supplementary Material. Figure A.3 shows the resulting average scattering factor as a function of the applied field angle for varying energy product $m B$. At an energy $m B$ well above $40 \mathrm{kT}$, the angular dependence approaches a $1-\sin (\theta)$ relationship.

Assuming a dipole moment of $0.25 \mathrm{fA} \mathrm{m}^{2}$ as reported in our earlier work 234], $m B=40 \mathrm{kT}$ corresponds to a field of about $0.7 \mathrm{mT}$. Therefore fields in the order of a few mT may be sufficient to obtain the maximum value of $C_{\mathrm{mag}}$. 


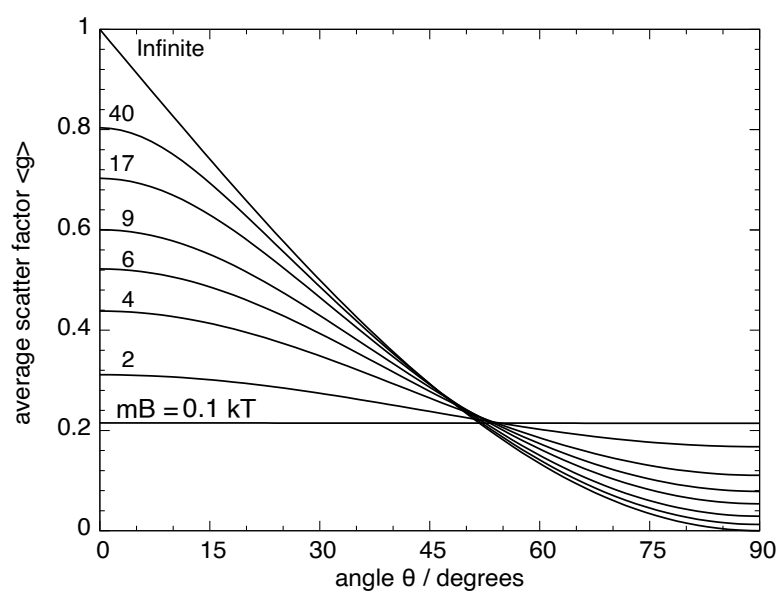

Figure A.3: Calculation of the average scatter factor as a function of the angle of the field with respect to the light incidence, for varying values of the product of the magnetic moment of the magnetosome chain $m$ and the applied field $B$, in units of $k T$ at room temperature. When all bacteria are perfectly aligned $(m B / k T=\infty)$, the average scatter factor is inversely correlated to the projection cross section of the bacteria on the light path $(g=1-\sin (\theta))$. At lower fields, the loss of alignment reduces the angular dependence, which disappears for $m B<k T$.

When the field is removed, the scatter factor $g_{0}=0.2146$. In this case the intensity on the detector $I_{0}=g_{0} I(0)+\left(1-g_{0}\right) I(90)$, which we can relate to the average $O_{\mathrm{D}}$ of the suspension

$$
\begin{aligned}
O_{\mathrm{D}}= & \log \left(\frac{I_{\mathrm{ref}}}{I_{0}}\right)= \\
& -\log \left(g_{0} 10^{-O_{\mathrm{D} \|}}+\left(1-g_{0}\right) 10^{-O_{\mathrm{D} \perp}}\right)
\end{aligned}
$$

In the above we ignored the disturbing force caused by the flagella. Flagellar motion is complex, so the disturbing force is difficult to calculate. We know however that in natural conditions, magnetotactic bacteria can use the earth magnetic field of about $50 \mu \mathrm{T}$ to navigate. In this low field $m B$ is only $3 \mathrm{kT}$. If the stochastic energy provided by the flagella is much larger than this value, the bacteria would not be able to follow the field. This suggests that for fields in the order of $\mathrm{mT}$, flagellar motion can be ignored.

\section{A.3 Method}

The MagOD system is an alternative for the modified commercial optical density meters that are currently used in magnetotactic bacteria research. It should therefore use compatible cuvettes and have comparable specifications. The 


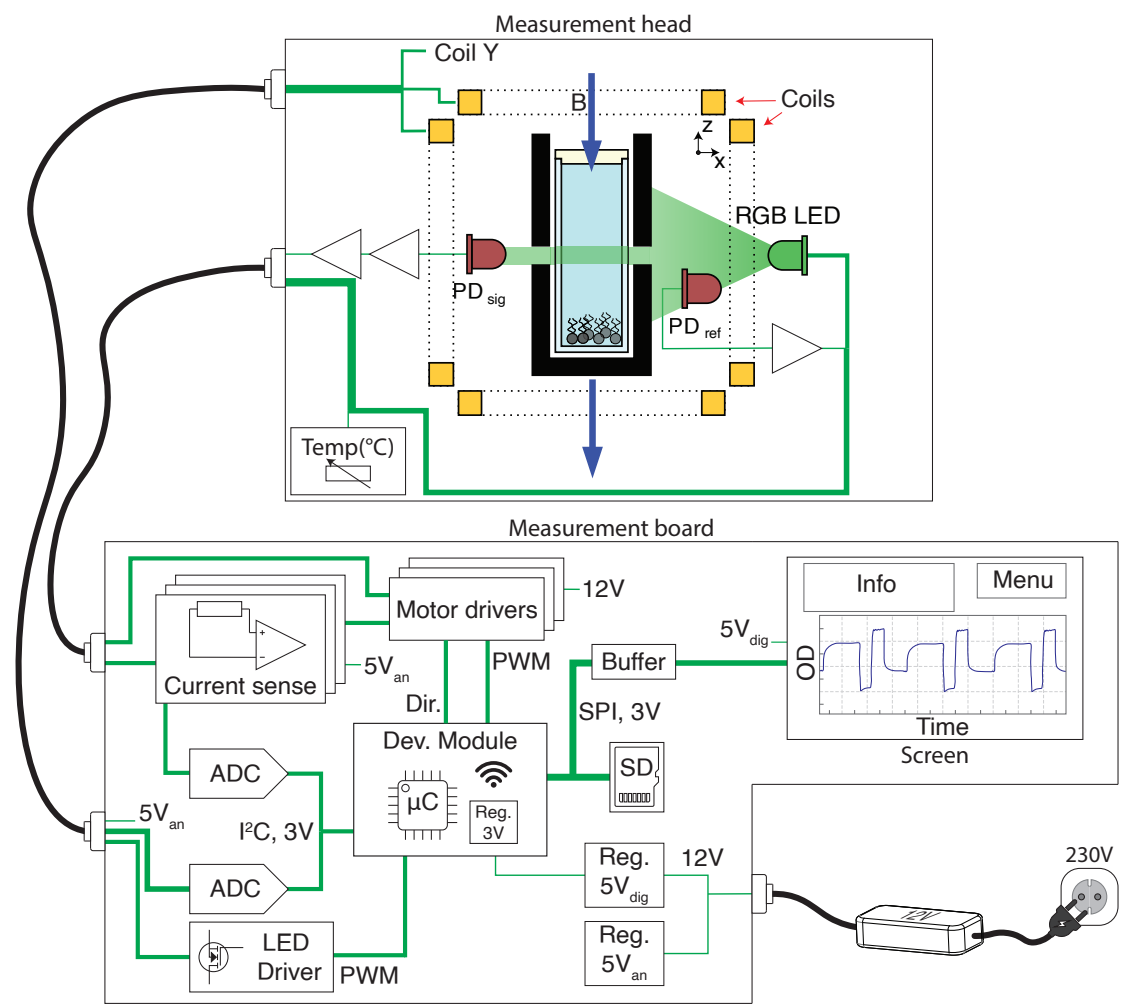

Figure A.4: Diagram of the system: Measurement head: LED, refdiode, diode, amplifier stages, coils, cuvette, bacteria. Measurement board: outlet to 12 supply, $12 \mathrm{~V}$ to $5 \mathrm{~V}$ analog and digital, microcontroller, AD converter, LED driver, motor shields, current sense, HDMI cable, coil cable, SD card, WIFI. User interface: resistive touch screen. Measurement board and user interface reside in the same housing.

preferred wavelength at which absorbance is measured is in around $600 \mathrm{~nm}$ and maximum absorbance is approximately 1.4 225]. Intensity variations due to change in direction of the magnetic field can be as high as $200 \%$, but values as low as $2 \%$ are reported [224]. Fields up to $70 \mathrm{mT}$ are applied [220], but there are indications that saturation occurs already at $2 \mathrm{mT}[224]$. As requirements for our design we therefore would like to have a wavelength of $600 \mathrm{~nm}$, absorbance range of at least 1.5, intensity resolution better than $1 \%$ and magnetic field above $2 \mathrm{mT}$.

The MagOD has two main components, see figure A.4. The cuvette filled with the sample to be investigated is inserted into the Measurement head that holds the light source and photodetector circuit boards, the three coil sets and additional sensors (such as temperature). The measurement head is connected to the Measurement board that holds the analog-digital converters, the drivers 
for the magnetic field generation and the light source. On the measurement board a micro-controller is mounted, which is connected over the board to the analog-digital converters, the data storage card and a touchscreen.

\section{A.3.1 Measurement head}

We designed the measurement head as compact as possible to keep the volume and power consumption low. The dimensions of the standardized cuvette $(12.5 \mathrm{~mm} \times 12.5 \mathrm{~mm} \times 45 \mathrm{~mm})$ determine the size of the coil system, which essentially sets the outer dimensions of the measurement head. The circuit boards for the light source and sensors are embedded inside the coil system, with sensors located as closely to the cuvette as possible.

\section{Mechanical}

Since the measurement head carries all components, it is a complex structure that has to be modified regularly to adapt for changes in component dimensions and added functionality. Therefore we decided to realize the structure by 3D printing, so that modifications can be easily implemented. Printing in metal still is prohibitively expensive, so the measurement head itself cannot act as electromagnetic shielding. Instead, shields will have to be implemented on the circuit boards. However, it is possible to 3D print in black nylon, so that the photodetector is shielded from external light and the parts can be easily disinfected using a $70 \%$ ethanol/water solution.

The measurement head consists of over a dozen parts. The design is parameterised using the open source OpenSCAD language, so that dimensions can be easily changed. The source files are available on Thingiverse.com

\section{Coil system}

We have the choice between permanent magnets or electromagnets with or without cores to apply a magnetic field. Since the field to be applied is relatively low, electromagnets without cores provide the simplest solution. The field is directly proportional to the current and there is no hysteresis, so no additional magnetic field sensors are required. The disadvantage of not having a core is it that the maximum field is limited to a few mT. Higher fields can only be applied for short periods of time, limited by coil heating.

The magnetic field is generated by three sets of two coils located on either side of the sample. The dimensions of the coils are more or less defined by the cuvette height, but we can choose the wire diameter to optimize the number of windings $N$. The field in the coil is proportional to the product of the current $I$ and $N$. The resistance $R$ of the coil scales approximately with $N^{2}$ for fixed coil dimensions. Therefore, the power dissipated in the coils $\left(I^{2} R\right)$ is relatively independent on the number of windings for a given field strength. The inductance of the coil $L$ scales 
Table A.2: Example of coil specifications.

\begin{tabular}{|c|c|c|c|}
\hline Jantzen Audio coil nr. & 1235 & 0996 & \\
\hline Wire gauge & 18 & 22 & AWG \\
\hline Wire diameter & 1.0 & 0.64 & $\mathrm{~mm}$ \\
\hline Resistance & 21 & 53 & $\mathrm{~m} \Omega / \mathrm{m}$ \\
\hline Inner diameter & 42 & 42 & $\mathrm{~mm}$ \\
\hline Outer diameter & 57 & 53 & $\mathrm{~mm}$ \\
\hline Height & 21 & 21 & $\mathrm{~mm}$ \\
\hline Inductance & 0.94 & 2.9 & $\mathrm{mH}$ \\
\hline Resistance & 0.5 & 2.1 & $\Omega$ \\
\hline Cut-off frequency & 85 & 115 & $\mathrm{~Hz}$ \\
\hline Winding 2 & $80(3)$ & $140(7)$ & \\
\hline Current for $1 \mathrm{mT}$ & $0.4^{3}$ & 0.54 & $\mathrm{~A}$ \\
\hline Voltage for $1 \mathrm{mT}$ & 0.44 & 1.1 & $\mathrm{~V}$ \\
\hline Power & 0.4 & 0.5 & $\mathrm{~W}$ \\
\hline
\end{tabular}

with $N^{2}$, so the cut-off frequency (proportional to $R / L$ ) is also fairly independent on the choice of the coil wire diameter. The choice for wire diameter is therefore mainly determined by the availability of power supplies, specifications of $\mathrm{H}$ Bridges and current ratings on connectors. Table A.2 shows the specifications of two commercially available coils (Jantzen Audio 000-1235 and 000-0996) as an example. The number of turns were estimated from the coil resistance (using literature values for wire resistance) and the coil inductance [238]. The MagOD implementation used in this publication incorporates the coil with the larger number of windings (996) to benefit from the substantially lower currents, but at the expense of a slightly higher cut-off frequency and power consumption.

\section{Temperature sensor}

Electromagnets - especially those without cores - produce heat as a byproduct of the magnetic field. In the absence of active cooling the temperature of the sample under investigation can raise quickly. This is especially a problem when working with micro-organisms. Therefore, it is important to monitor the temperature of the cuvette. The best option would be to instert a temperature sensor into the cuvette. This method however is cumbersome and carries the 
risk of exposing the sample to the outside air. The temperature of the coils can be estimated from their resistance, but that would overestimate the temperature of the cuvette. Therefore, we chose to mount a simple NTC temperature sensor in the housing, as closely as possible to the cuvette.

\section{Light source}

Ideally, the absorption pattern of a specimen is measured over a large range of wavelengths. Most optical density meters use a wide spectrum Xenon flash light in combination with a monochromator. This is a rather power-hungry, bulky solution $(>10 \mathrm{~W}, 20 \mathrm{~mm})$, and overkill for the observation of magnetotactic bacteria. Instead, we chose an RGB LED as source. These LEDs are simple to control, can be mounted closely to the cuvette, can be operated in continuous mode and can be easily adjusted in intensity using pulse width modulation (PWM). The wavelength however cannot be chosen continuously, but the wavelength spectrum is determined by the LED type. Moreover, the wavelength bandwidth per color is rather large ( $25 \mathrm{~nm}$ compared to $5 \mathrm{~nm}$ for monochromators). Finally, the light intensity of a LED is small compared to Xenon lights or lasers. Based on manufacturers data, we estimate that in our current implementation the LED power is approximately $0.2,0.1$ and $0.7 \mu \mathrm{W} / \mathrm{mm}^{2}$ for 645 (red), 520 (green) and $460 \mathrm{~nm}$ (blue) light respectively. This however is sufficient for most suspensions of magnetotactic bacteria.

The LED has a non-diffuse housing such that the light output in the direction of the sample is optimal. The LEDs can easily be exchanged for e.g. a yellow or UV LED, since they are mounted on a separate board.

The LED is mounted in common anode configuration such that it can be driven by NPN mosfets and the supply difference between the LED $(5 \mathrm{~V})$ and the microcontroller $(3 \mathrm{~V})$ does not cause an issue. The frequency of the PWM signal is well above the cut-off frequency of the photodetector amplifier. The brightness of LEDs decreases with time. To monitor the LED intensity, a photodiode is placed in close vicinity, before the light enters the cuvette.

\section{Photodiode}

The detection of the light passing the cuvette can be done with photo-multiplier tubes, avalange photodiodes and silicon photodiodes. Photo-multipliers have very high sensitivity, but are quite bulky, require high voltages and perform less well at long wavelengths. Avalange photodiodes are also very sensitive, but suffer from non-linearity, noise, high temperature dependence, and also require high voltages to operate. Since the transmission of light through most magnetotactic bacteria suspensions is hight and we work at low acquisition frequencies, the silicon photodiode sensitivity is sufficient. We can take advantage of its small form factor, linearity and ease of operation. We used the more light-sensitive 
large-area photodiodes (PD) to boost sensitivity. The diode is operated in photovoltaic mode. In this mode the bias voltage is zero, so the dark current, which is highly temperature sensitive, is minimized.

In the current MagOD implementation, the PD current is amplified by a two-stage opamp circuit since the signal is too weak for a single stage amplifier. The first stage is a current to voltage converter. A low noise JFET opamp is applied, because this type of opamp has a low input current offset, which reduces DC errors and noise at the output. The first stage has the largest amplification in order to minimize the amplification of noise. The amplifier circuit is located right behind the $\mathrm{PD}$ inside a EM-protective casing, so that noise picked up by the cabling to the main board is not amplified and interference is minimized.

\section{A.3.2 Measurement board}

Placing the photodiode amplifier directly behind the photodiode is a good way to suppress interference. We have the option to transport the amplified photodiode signal directly to the measurement board or to include the AD converter next to the amplifier in the measurement head and convert the analog to a digital signal. The analog option has the advantage of a small form factor for the circuit board and better access for testing. The digital option will suffer less from interference and allows for simpler cabling. Since the current implementation of the MagOD system is very much a development instrument, we chose to move the $\mathrm{AD}$ converters to a separate measurement board, together with the microprocessor and other peripherals.

\section{AD converter}

The measurement board has two analog-to-digital (AD) converters to read out the various analog signals on the system. As the measurements are normally performed on a larger timescale, we chose converters which are able to perform measurements with a sampling rate up to $860 \mathrm{~Hz}$ and have integrated anti-aliasing filters. A resolution of 16-bit provides an upper limit to the absorbance of 4.8, which is more than sufficient. In practise, the absorbance range is limited by stray light scattering around the sample.

The AD converters have a free-running mode, which performs measurements at an internally defined clock rate. A data-ready pin functions as an external interrupt such that the microcrontroller can be freed for other tasks while waiting for the $\mathrm{AD}$ converter to finalize its acquisition step.

\section{Microcontroller}

Since data acquisition rates are low, the MagOD system can be easily controlled by a microcontroller $(\mu \mathrm{C})$. We can benefit from recent developments in cheap, versatile $\mu \mathrm{C}$ development platforms. Rather than embedding the microprocessor 
directly on the the electronic board, we chose to include the $\mu \mathrm{C}$ as a development board. This way, the system can be easily assembled, debugged and repaired.

The current implementation of the MagOD instrument is built around an ESP32 development board. The ESP32 $\mu \mathrm{C}$ has several characteristics that make it very suitable for this application: it has a small form factor, a fast 32-bits dual core processor operating at $240 \mathrm{MHz}, \mathrm{WiFi}$ and Bluetooth and several peripheral interfaces such as SPI and $\mathrm{I}^{2} \mathrm{C}$. This $\mu \mathrm{C}$ is very popular, resulting in a large amount of dedicated libraries, examples and discussions in internet fora. Additionally, there is a plugin for the Arduino IDE and many libraries are natively compatible, so inexperienced developers can start with little effort.

\section{Display}

A resistive touch screen is added to conveniently control the system either with or without protective gloves. Additionally, the screen provides the user with information on the current and past states of the measurement and levels of the signals. Line drivers on the main board ensure communication is reliable.

\section{Storage}

The storage of the acquired data and the recipes is done on a Secure Digital (SD) card. These cards are readily available in a variety of capacities, are widely applied in DIY projects, and are replaceable in case of a damaged card. The $\mathrm{SD}$ card can be interfaced to the $\mu \mathrm{C}$ in the SPI, the 1-bit SD, and the 4-bit SD mode. While the data transfer is faster using the 4-bit SD mode, we chose the SPI mode since it is well supported and the write speed is sufficient for our purpose. However, the write time to an SD card over an SPI interface using the ESP32 micro-controller is unpredictable, with SD card induced peaks in write time of at least $50 \mathrm{~ms}$. Fortunately, the ESP32 has two cores, so unpredictable processes like access to the SD card, reaction to touch screen input and WiFi file transfers can be moved to a separate core.

\section{Current drivers}

The current through the coils needs to be controlled to obtain a specific magnitude of the magnetic field. We use PWM and benefit from the fact that the high inductance of the coil provides a low frequency cut-off filter for free. The use of PWM minimizes power dissipation in the supply, but results in a current ripple and consequently a ripple in the magnetic field. This ripple can be suppressed by choosing a sufficiently high PMW frequency. We use commercial motordrivers because these are specialized to drive high currents through a coil in two directions based on a simple two-wire control. The currently employed drivers work with frequencies up to $20 \mathrm{kHz}$, suppressing the ripple by at least a 
factor of 100 . The drivers can be interchanged by alternative motor drivers with similar capabilities.

The magnetic field is linearly dependent on the current. However, the current is not linearly dependent on the PWM duty cycle, as the internal resistance of the coil will vary due to temperature changes. A precise measurement of the current is necessary to close the loop and to assess the applied magnetic field. Therefore, a shunt resistor is placed in series with each coil. The voltage drop over this resistor is amplified using a current sensing amplifier and digitized with the AD converter. The measured signal can either be used to determine the true current, or applied in a feedback loop to compensate for coil heating.

\section{Power Supply}

The measurement board electronics operate at low voltages ( 3 or $5 \mathrm{~V})$. However, the magnetic coil system is preferably operated at higher voltages, to limit the currents and subsequent requirements for cabling and connectors. For reasonable winding wire diameters, the currents are in the range of a few ampere and the resistance of the coils in the order of a few ohm. Therefore, we selected for the main on-board supply $12 \mathrm{~V}$, for which a wide range of external power supplies are available and which even allows for operation from a car battery while in the field.

In the current MagOD implementation, the three coil sets have a combined resistance of $4.2 \Omega$ at room temperature. The maximum current is close to $3 \mathrm{~A}$ with $12 \mathrm{~V}$. This maximum current through each coil set simultaneously would require a power supply of at most $120 \mathrm{~W}$.

The analog and digital circuitry have a separate $5 \mathrm{~V}$ supply line to prevent noise originating from the switching nature of the digital circuitry to interfere with the measurement. The analog $5 \mathrm{~V}$ supply is built using an ultra low noise linear regulator, whereas the digital $5 \mathrm{~V}$ is built with a switching regulator. The latter is more efficient, but produces inherently more electronic noise. The $3 \mathrm{~V}$ needed for the $\mu \mathrm{C}$ originates from a linear regulator integrated on the development board.

\section{Enclosure}

The device is enclosed in a lasercut plastic housing. The choice for plastic was made since it does not block the WiFi signal. We do not have to worry about interference signals, since the measurement signal is amplified in the measurement head and the unshielded sections of the leads to the AD converter are kept very short.

The design is made such that no extra materials are needed for assembly. Additionally, the parts can be manufactured with a $3 \mathrm{D}$ printer. The source code for the enclosure design is available on github. 


\section{A.3.3 Cabling}

While designing the MagOD system, it was envisioned that measurements could take place inside controlled environments, such as incubators and fridges. Therefore the system was separated into two parts, connected by cabling. Components that did not need to be on the measurement head were moved to a separate module. This approach has the disadvantage of the additional complication of cabling and connectors. To mitigate this problem, we chose to resort to commercially available cabling where possible.

For the communication with the amplifier boards in the measurement head we chose an HDMI cable. These contain shielded twisted pair cables with separate non-isolated ground line that are perfectly suited to transmit analog signals with low interference (5V, signal, ground). The HDMI interface progressed through several standards. The HDMI2.1 + Internet standard has five shielded twisted pair that can be used for measurement signals (for instance 3 photodiodes, NTC and Hall sensor) and four separate wires that can be used for control signals (3 LEDs). The connectors on the main board, amplifier boards and motor drives are standard Molex connectors. The coils are connected to standard measurement leads with banana connectors. The connection from the banana plugs to the measurement head is based on a Hirose RP 6-pole connectors, which is the only cable that cannot be purchased in assembled form.

\section{A.3.4 Software}

Most modifications to the MagOD system will be at the software level, which will primarily be done by students. Generally, (electrical) engineering students and many hobbyists are skilled in programming of Arduino development boards. Therefore, the microprocessor (ESP32) was programmed in the same way as an Arduino project, using $\mathrm{C}++$ and the native Arduino IDE both as compiler and uploader. This has the major advantage of a neglible entry barrier for inexperienced microprocessor programmers.

The disadvantage of the Arduino IDE is that it is not very suitable for larger projects. The current implementation is already exceeding 5000 lines of code. To partially relieve this issue, the code was set up in a highly modular way to assist new programmers in navigation, using only one main source file (.ino, .h) of 1000 lines, and a dozen local library source files ( src/*.cpp) for e.g. screen access, readout of the ADC, writing to Flash memory, Wifi access. The source code can be found on github.

The data is collected on the SD card and transferred over WiFi is in a format that can be easily imported and displayed in a spreadsheet program. For more advanced analysis, python scripts are available on github. 


\section{A.4 Results}

We analyse the performance of the current implementation of the MagOD, and compared it to a commerical spectrophotometer in the first part of this results section. To illustrate the possiblities of the new instrument, we give three examples in section A.4.2

\section{A.4.1 Performance}

Several iterations of MagOD systems have been realised based on the design considerations of section A.3. We expect that more iterations will follow, not only by our team but also by others in the field of magnetotactic bacteria. The most recent implementation can be found online at github. We measured the performance on the current implementation of the MagOD meter (version 2) with respect to the optical and magnetic components to provide a baseline for future improvement.

\section{LED and Photodetector}

Photodectector sensitivity The MagOD systems is equipped with a three color LED which allows selection of three wavelenghts (peak intensities at 645, 520 and $460 \mathrm{~nm}$ ), either individual or in combination. The LEDs are indvidually driven by a PWM voltage to adjust their intensity, for instance to match the transmission of light through the liquid in the cuvette. A reference photodiode is mounted adjacent to the LEDs which captures a small fraction of the LEDs' light, to monitor variations in the emitted light intensity. Figure A.5 shows the signal of the detector and reference photodiodes as a function of the average LED power, for the three different wavelenghts. The light pattern is shown in figure A.18 in the appendix, with a video in the Supplementary Material.

Space restrictions forced us to design the two stage amplifier such that the output decreases with increasing LED power. The reference photodiode, which has only one amplifier stage, has an increasing output with increasing intensity.

The relation between output voltage and intensity is linear for the red and green LED, but not for the blue LED at higher intensities. Measurement with liquids of different absorbance (figure A.19 in the appendix) confirm that the senstivity to the blue light drops at high intensity of the incident light.Therefore, the blue LED should only be used for accurate absorbance at low indicent power (signal above $2 \mathrm{~V}$ ). At low intensity, the sensitivities of the red and blue channels are approximately equal, and twice as high as the green channel for the chosen combination of LED and photodetector. The sensitivity of the reference photodiode to red and blue is however clearly different. This again may be related to the placement of the diodes in the LED housing. 


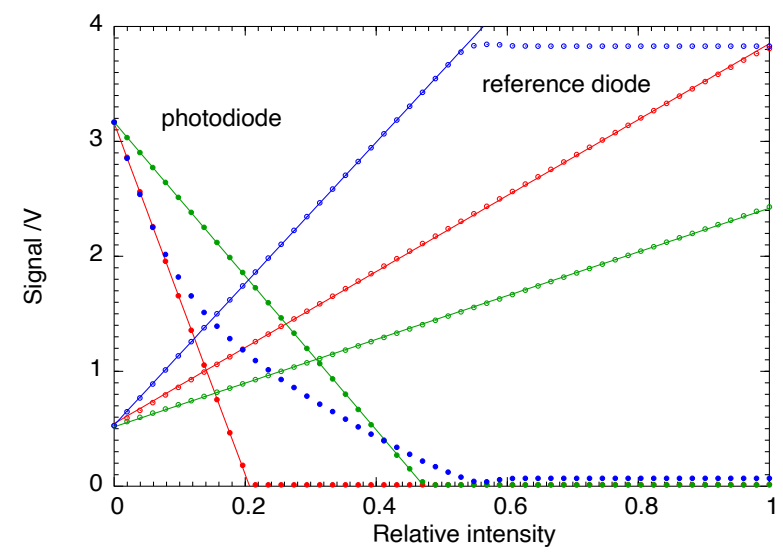

Figure A.5: Photodiode and reference diode signal versus the LED dutycycle (proportional to effective power). Note that the signal on the photodiode decreases with increasing light intensity, due to the particular two-stage design of the amplifier. The reference diode amplifier is based on a conventional one-stage design. The photodiode signal is linear with the duty cycle for the red $(645 \mathrm{~nm})$ and green $(520 \mathrm{~nm})$ LED, but not for the blue $(460 \mathrm{~nm})$ LED. Similarly, the reference diode signal is linear with the duty cycle of the red and green LEDs, but not for the blue. The fit parameters for the linear fits are shown in table A.3

Table A.3: Linear fits to measurements of figure A.5 The blue LED has a non-linear response and is not tabulated.

\begin{tabular}{|c|c|c|c|c|c|}
\hline \multicolumn{2}{|c|}{ LED } & \multicolumn{2}{|c|}{ photodiode } & \multicolumn{2}{|c|}{ reference diode } \\
\hline $\begin{array}{l}\text { Peak } \\
\mathrm{nm}\end{array}$ & $\begin{array}{c}I_{\max } \\
\mathrm{mA}\end{array}$ & $\begin{array}{l}\text { offset } \\
\mathrm{V}\end{array}$ & $\begin{array}{c}\text { slope } \\
\mathrm{V} / I_{\max }\end{array}$ & $\begin{array}{l}\text { offset } \\
\text { V }\end{array}$ & $\begin{array}{c}\text { slope } \\
\mathrm{V} / I_{\max }\end{array}$ \\
\hline 645 (red) & $20(2)$ & $4.19(5)$ & $-48(1)$ & $0.554(1)$ & $2.105(1)$ \\
\hline 520 (green) & $20(2)$ & $4.10(2)$ & $-22.7(2)$ & $0.553(1)$ & $1.391(1)$ \\
\hline
\end{tabular}

The linear fits to the data are listed in table A.3 The offsets are in agreement with the manufacturers specification of the ADS115 of $4.096 \mathrm{~V}$.

Absorbance validation To validate performance with respect to standard photospectroscopy measurements, we compared the MagOD system with a commercial optical density meter (Eppendorf BioPhotoMeter Plus). Figure A.6 shows the absorbance $\left(O_{\mathrm{D}}\right)$ relative to water as a function of the wavelenght of the light for a range of dilutions of a suspension of magnetic nanoparticles (FerroTec EMG 304). The transmission of light measured by the MagOD meter was averaged for a range of photodiode intensities ranging from zero to saturation. For the blue LED however, care was taken to measure only at low intensities, where the response is linear (see figure A.5.

As expected, the absorbance increases with increasing nanoparticle concen- 


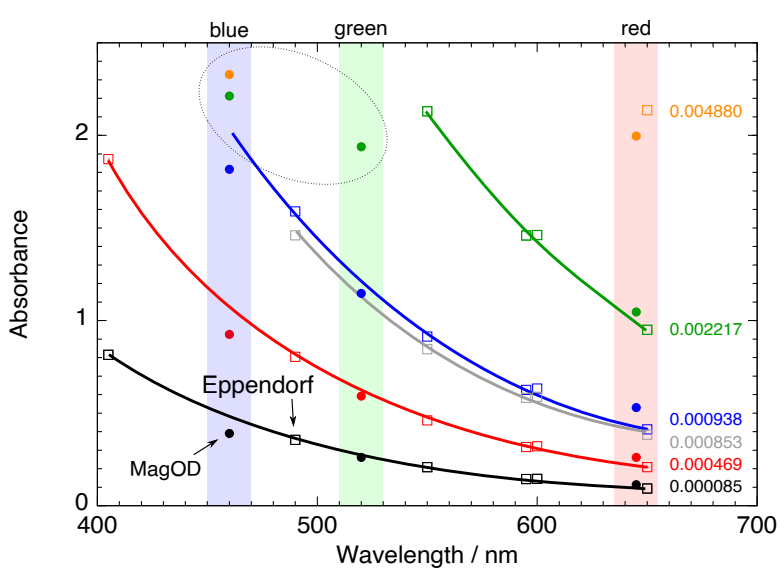

Figure A.6: Absorbance relative to water measured with the MagOD meter (closed circles in the colored bands) for the three LEDs, compared to the OD measured by an Eppendorf BioPhotoMeter (open squares) measured as a function of the wavelength. A range of dilutions of a water based ferrofluid was used (FerroTec EMG 304, the dilution factor is indicated on the right). The datapoints for every dilution are indicated by a line to guide the eye. The difference between the MagOD meter and the commercial instrument is larger than the estimated measurement error, but less than 0.2 for absorbances below 2. Above this value, the estimate is unreliable (points inside dotted loop). The absorbance of the blue LED is systematically lower than the commercial instrument. The maximum absorbance measured was 1.82 , which is slightly lower than the commercial instrument $(2.14)$. The uncertainty on the measurements is smaller than the symbol size, and is omitted for clarity.

tration (indicated on the right of the graphs). The absorbance increases with decreasing wavelength, which is in agreement with the observation that the solution has a brown appearance. Care was taken to determine the accuracy of the measurement as accurately as possible. At this precision, it is clear that the novel MagOD meter and the commercial instrument deviate. This deviation is however never larger than 0.2 for absorbances below 2. Above this value, the deviation becomes considerable (datapoints inside dotted loop), probably because of light scattering onto the photodetector through other paths.

The blue LED seems to systematically underestimate the absorbance, which may be related to the fact that the response of the detector is ill defined. The maximum absorbance is comparable to the commercial instrument. We therefore conclude that the instrument works satisfactory as a conventional absorbance meter, especially the red and green channels.

Time response and noise level The ADS1115 AD converter has a maximum sampling rate of 860 samples/s, which means a sampling time of $1.2 \mathrm{~ms}$. Figure A.7 shows a time sequence of the sampled photodiode signal at that rate. The red LED was switched on and modulated from 46 to 47 bits on a full 
range of 255 (relative intensity approximately 0.18 ) every 250 samples. The total acquisition of 1300 samples took $4023 \mathrm{~ms}$, so the effective sample-rate was only $323 \mathrm{samples} / \mathrm{s}$. The reduction in data-rate is due to communication overhead with the AD converter, and can be optimized.

The data in figure A.7 shows two clear levels, without any measurement points in transition from one to the other. Therefore, we can safely conclude that the response of the MagOD meter at the highest sample-rate is better than $3.1 \mathrm{~ms}$. This is in agreement with the filter applied in the feedback loop of the amplifier, which has a $-3 \mathrm{~dB}$ point at $800 \mathrm{~Hz}(1.25 \mathrm{~ms})$.

The ADS1115 has an internal filter that matches the bandwidth, which can be selected from discrete values of $8,32,64,128,250,475$ and 860 samples/s. Therefore, the noise should decrease at lower sample-rates. Figure A.8 shows the standard deviation of 1000 samples, which is equal to the RMS noise, as a function of the sample-rate. As expected, the noise increases with increasing sample-rate, but much steeper than can be expected from a white noise spectrum (noise proportional to the square root of the bandwidth). There is a strong jump in noise above 64 samples/s. Most likely, this is caused by the presence of a $50 \mathrm{~Hz}$ cross-talk signal. At 64 samples/s and below, the noise is in the order of 1 bit or $125 \mu \mathrm{V}$. Since the full range of the detector circuit is $3.1 \mathrm{~V}$, this corresponds to a dynamic range of $88 \mathrm{~dB}$ or a theoretical upper limit to the detectable absorbance of 4.4. This compares very favourably to the commercial Eppendorf system, which has a resolution in OD of $1 \times 10^{-3}$ on full range of approximately 2. Assuming that the noise level of the Eppendorf system is comparable to the resolution, this would correspond to a dynamic range of only $53 \mathrm{~dB}$.

At 64 samples/s the noise level is $16 \mu \mathrm{V} / \sqrt{\mathrm{Hz}}$. Spice simulations indicate that the theoretical noise level of the amplifier is in the order of $0.5 \mu \mathrm{V} / \sqrt{\mathrm{Hz}}$, so we have not yet reached the full potential of the electronics.

\section{Magnetic field system}

Figure A.9 shows the magnetic field in the center of the system, as a function of the current through each of the three coil sets. The coils generate approximately $2 \mathrm{mT} / \mathrm{A}$, with around $5 \%$ variation between the coils. The maximum field that can be generated is slightly higher than $5 \mathrm{mT}$ at full current of approximately $2.5 \mathrm{~A}$. The pulse width of the modulation of the driver circuits can be set with a resolution of at maximum $16 \mathrm{bit}$, corresponding to a theoretical field resolution of about $70 \mathrm{nT}$. In practise, we operate the PWM at 8 bit resolution which gives a setpoint resolution of about $20 \mu \mathrm{T}$.

Since we drive the coils with a PWM signal, the current through the coils is not constant but follows the modulation frequency. At zero and maximum current, the ripple is absent. The ripple has a maximum at $50 \%$ duty cycle. The filtering action of the coil system dampens the modulation. At a PWM drive 


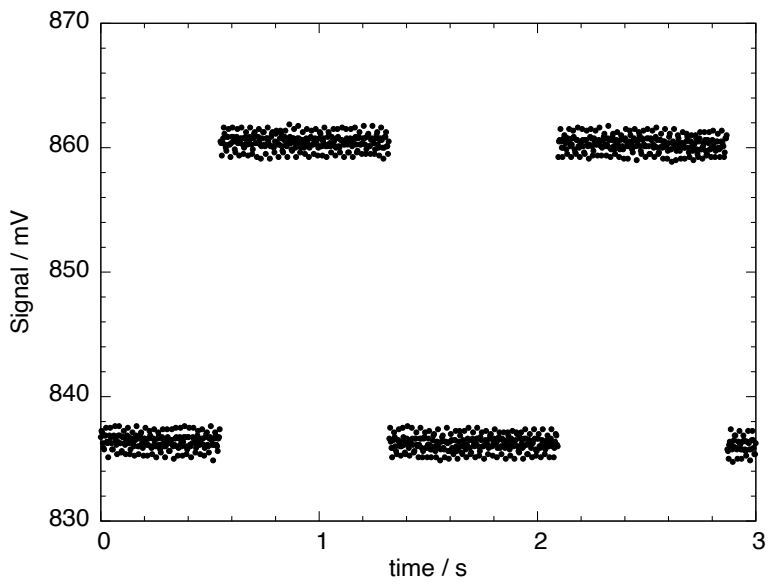

Figure A.7: The detector photodiode voltage sampled by the AD converter at a rate of 860 samples/s, while the red LED power is modulated by $0.4 \%$. The effective sample-rate was 323 samples/s. No transitions between the levels can be observed, so the time response of the detector photodiode is better than $3.1 \mathrm{~ms}$.

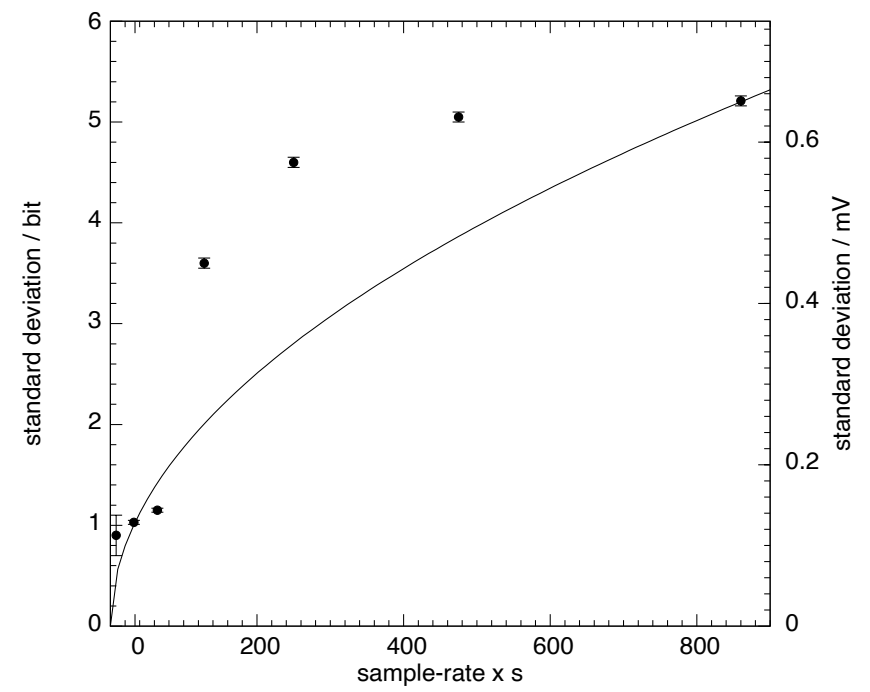

Figure A.8: Standard deviation (rms noise) over 1000 samples taken by the AD converter of the detector photodiode signal, as function of the sample-rate. The noise increases with increasing sample-rate, but not proportional to the square root of the bandwidth (solid line). Above 64 samples/s there is a strong increase in noise. 


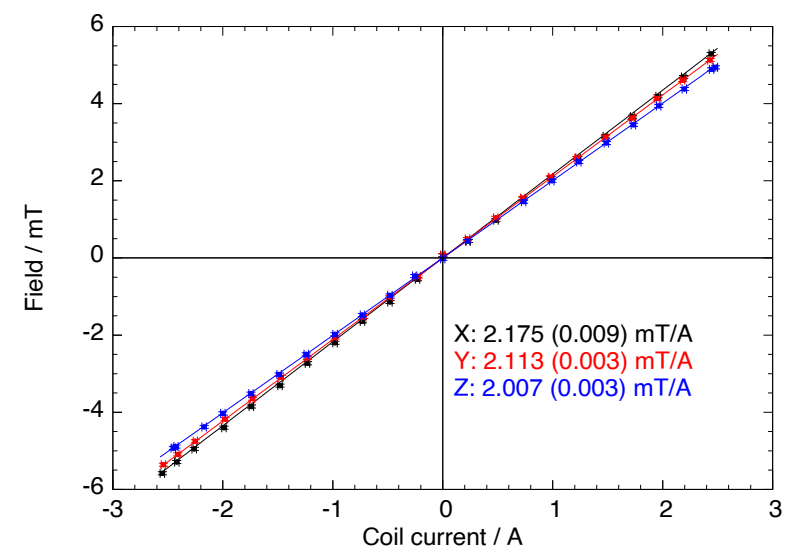

Figure A.9: Magnetic field in the center of the cuvette holder as a function of average coil current. The fields in $x, y$ and $z$ direction with a field to current ratio of 2.175(9), 2.113(3) and $2.007(3) \mathrm{mT} / \mathrm{A}$ respectively.

frequency of $20 \mathrm{kHz}$ and $50 \%$ modulation we measured a triangular current signal with a peak-peak amplitude of 24(2) $\mathrm{mA}$ on mean current of 1.2 A. Simulations considering only the LR nature of the coils, with a corner frequency of $115 \mathrm{~Hz}$, give a theoretical amplitude of $18 \mathrm{~mA}$, so there probably is some additional capacitive coupling. The current variation corresponds to a maximum field variation in the field of approxmately $50 \mu \mathrm{T}$ or $1.2 \%$.

At the maximum current of $2.5 \mathrm{~A}$, the coils dissipate about $13.1 \mathrm{~W}$ each. Since the coil system has no active cooling the heating of the sample area can be considerable at prolonged measurement times. An NTC temperature sensor mounted on the body of the measurement chamber to monitor the temperature. Figure A.10 shows the temperature rise of the NTC mounted on the chamber. We also measured the temperature in the chamber with a simple alcohol thermometer for comparison. The temperature of the coils can be estimated from the increase in coil resistance assuming the temperature coefficient of copper $(0.393 \% / \mathrm{K})$.

At a drive current of $0.5 \mathrm{~A}$ (field strength of $1 \mathrm{mT}$ ) the heating of the chamber is barely noticeable (about $1 \mathrm{~K} / \mathrm{h}$ ). The average temperature of the coils increases with approximately $8 \mathrm{~K} / \mathrm{h}$. At a drive current of $1.2 \mathrm{~A}$, the temperature of the coils increase by $21 \mathrm{~K}$. The temperature increase of the chamber is substantial, with an initial increase of approximately $0.25 \mathrm{~K} / \mathrm{min}$, flattening out at 7 to $8 \mathrm{~K}$ after $40 \mathrm{~min}$.

\section{A.4.2 Applications}

We present four experiments to illustrate the application of the MagOD meter in the analysis of magneto-tactic bacteria. We measure (1) the scattering of Magnetospirillum gryphiswaldense (MSR-1) as a function of their angle to the 

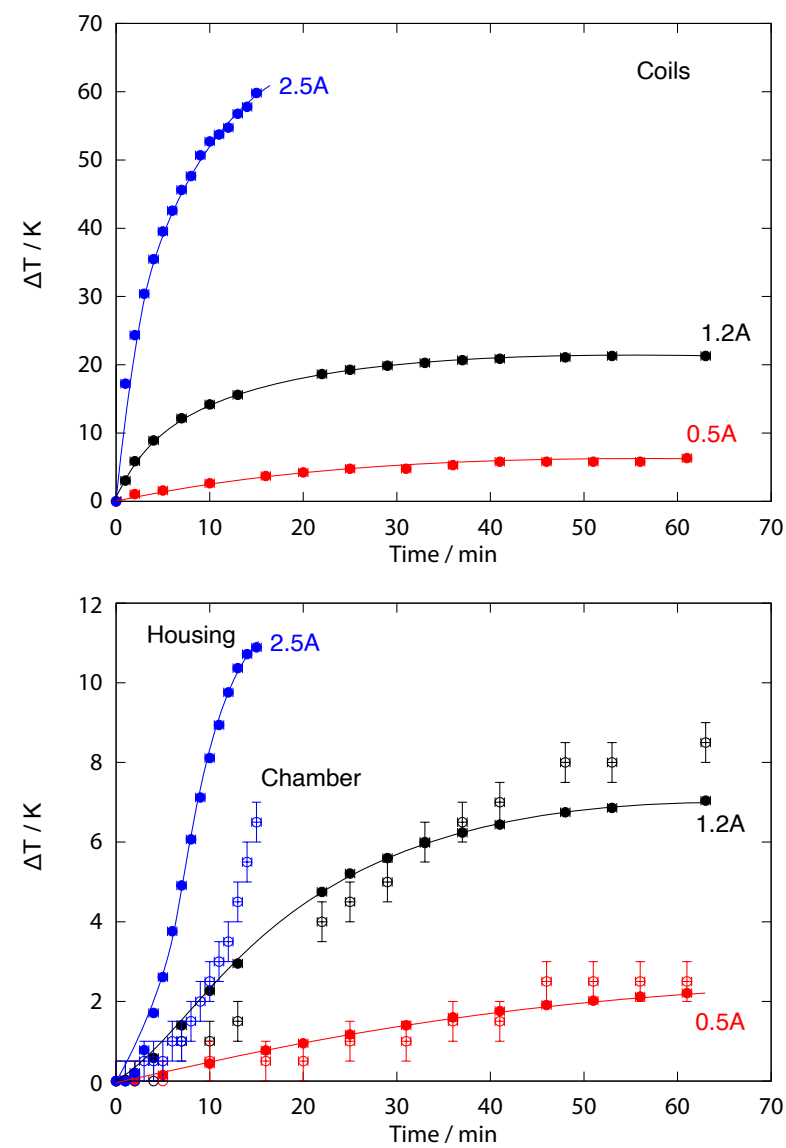

Figure A.10: Top: Temperature of the coil system as a function of time, for different drive currents. At the maximum drive current of $2.5 \mathrm{~A}$, corresponding to $5 \mathrm{mT}$, the coils heat rapidly and operation should be limited to a few minutes. Bottom: MagOD frame temperature (closed symbols) and air temperature in the chamber (open symbols) as a function of time. At a drive current below $1.2 \mathrm{~A}(2.4 \mathrm{mT})$ the temperature increase of the frame is limited to about $6 \mathrm{~K}$. 
incident light, (2) their rotational velocity as a result of a rotation of the external magnetic field on timescales of seconds and (3) the development of a culture over a period of several days. In the last experiment (4), we measured the velocity distribution of the unipolar Magnetococcus marinus (MC-1) as a function of time.

\section{Transmission as a function of angle (MSR-1)}

With the coil system of the MagOD meter we can apply a field in any direction in three-dimensional space. This allows us to study the transmission of light as a function of the orientation of the bacteria and check the model presented in section A.2.

For this, a cuvette with MSR-1 bacteria, grown according to [234], with an OD of approximately 0.1 was inserted in the MagOD system. We measured the intensity on the photodetector as a function of the angle of the magnetic field with steps of approximately $5^{\circ}$. The magnetic field varied with angle, but was always over $1 \mathrm{mT}$. As the optical density of the sample is continuously fluctuating due to activity and sedimentation within the cuvette, the measurement was repeated 20 times. The resulting curves were normalised to a range of 0 to 1 and averaged to obtain the the angle-dependent scatter factor $g(\theta)$ shown in figure A.11.

The simple inverted sine model of section A.2 fits surprisingly well. The strongest deviation is around the parallel alignment, which is understandable. The MSR-1 are not infinitely thin rods, but spirals. Therefore, the projected area will be less sensitive to variations in the angle around the long axis. Additionally, the culture of MSR will have a distribution in angles (due to Brownian motion and/or flagellar motion), which will 'smear out' the sharp corner at $\theta=0$. An illustration of this effect for $m B=60 \mathrm{kT}$ is shown in the red curve, which still does not fit the measurement very well. It seems therefore likely that the actual bacteria shape, and maybe also their distribution, should be included in the model.

\section{Response as function of field strength and time (MSR-1)}

In our lab, we most commonly perform measurements in which the sample of MSR-1 is subjected to a field switching between parallel and perpendicular to the light path, with varying field strengths. Figure A.12 shows the measured response for a set of field cycles 5 At a high field value of $3 \mathrm{mT}$ the field is switched from parallel to perpendicular after $10 \mathrm{~s}$. For the lower field value of $0.4 \mathrm{mT}$ we can take a longer reversal times since coil heating is no issue.

\footnotetext{
${ }^{5}$ In this measuremen, the absorbance is high (transmission of light is low) when the field is aligned along the light path. This measurement was performed with an older, single stage photodiode amplifier, unlike the measurement in figure A.5 made with the new amplifier that has an inverted response
} 


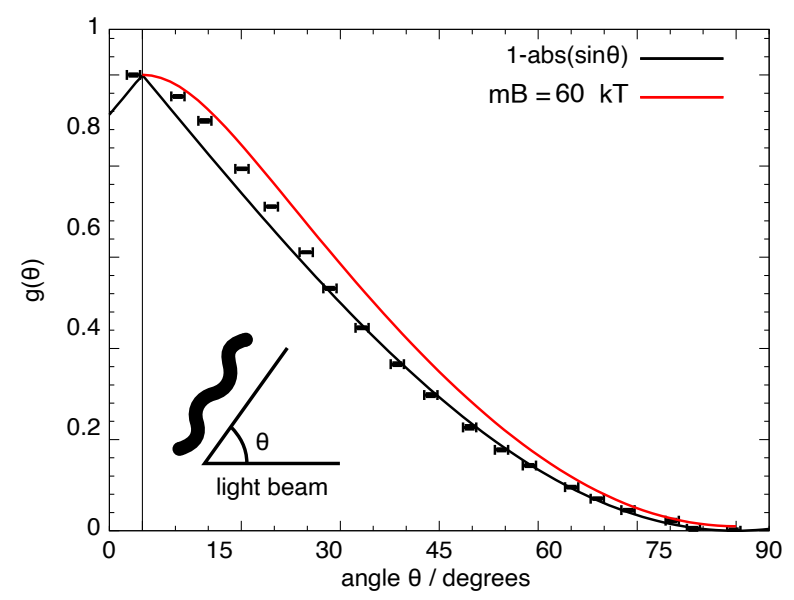

Figure A.11: The scattering of a culture of MTB is dependent on orientation of the external magnetic field; it is highest when the field (so also the bacteria) are aligned parallel with the light beam and lowest when the field is aligned orthogonal. By normalizing from $[0,1]$, we obtain the angle-dependent scatter factor $g(\theta)$ that can be relatively well approximated by a sine. The flattening of the curve around $0^{\circ}$ can be partly explained by additional brownian motion of the bacteria (red curve).

From the difference in detector signals, we can calculate $\Delta_{\text {OD }}$ using equation A.4. The signal of the growth medium without bacteria $\left(I_{\text {ref }}\right)$ was $301(1) \mathrm{mV}$, so we can calculate $C_{\text {mag }}$ using equation A.3.

The difference in transmission between in-plane and perpendicular alignment is higher at $3 \mathrm{mT}$ as compared to $0.4 \mathrm{mT}$. This is in agreement with the predicted increase of scatter factor with increasing field (Figure A.3). Figure A.13 shows the calculated difference in scatter factor as a function of the magnetic field, scaled to $k T / m$. From previous analysis of MSR-1 [234] we estimated that the mean magnetic moment $m$ of the magnetosome chain is $0.25 \mathrm{fAm}^{2}$, with a $10-90 \%$ cutoff of the distribution of 0.07 and $0.57 \mathrm{fAm}^{2}$ respectively. We can convert these ranges of moments to the energy ratio $m B / k T$ for the two difference field values. The ranges are indicated on the top axis of the graph by lines and the mean values by red circles on the red line. The predicted reduction between the average scatter factors (0.20) at the two field values is less than observed in figure A.12 (0.5). However, considering that we have a simplified model and our previous estimate of the magnetic moment may be different for the current sample, the agreement with the measurement is acceptable. Next to a decrease in step height, the time response also decreases with decreasing field. The time constant is estimated from a fit of equation A.5 to the data using the sum of squared errors criterion. The time constant of the transitions to $3 \mathrm{mT}$ is $1.7(5) \mathrm{s}$, which is approximately 13 times higher than the time constant of 5.4(8) s of the transition to $0.4 \mathrm{mT}$. The ratio is on the high side, but still within measurement 


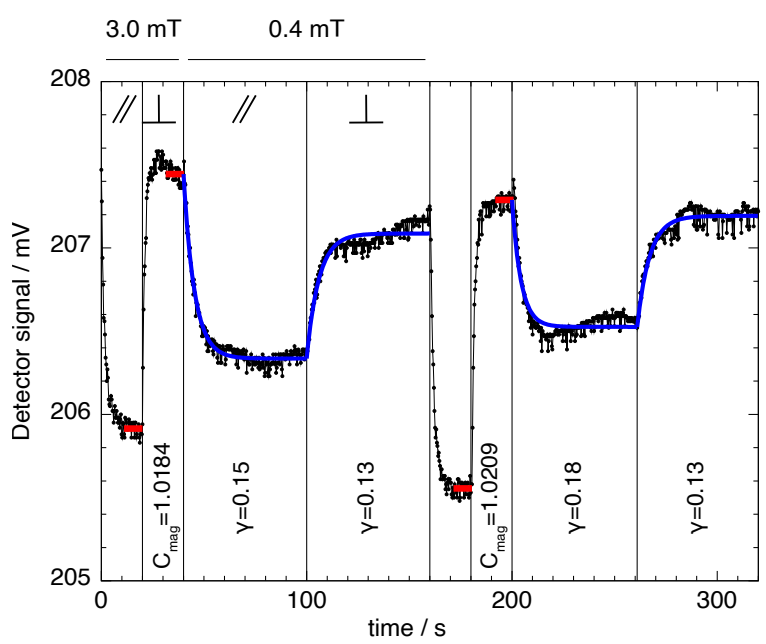

Figure A.12: Two cycles of a measurement sequence. The magnetic field is alternatively aligned parallel and perpendicular to the light beam. At high field $(3.0 \mathrm{mT})$, we can determine $\Delta_{\text {OD }}$ from the difference between the averages of the detector signals (red lines), from which we can calculate $C_{\text {mag }}$ (The signal of the growth medium without bacteria was $301(1) \mathrm{mV}$ ). The difference between the two directions of the field drops considerably at low field $(0.4 \mathrm{mT})$, whereas the response time increases. These low fields are suitable for estimation of the the time constant $\tau$ from a fit to an exponential (blue line). Using the field magnitude, we can calculate $\gamma(\mathrm{rad} / \mathrm{mTs})$.

error equal to the ratio of the fields, as predicted by the model of section A.2.

\section{Long term growth monitoring (MSR-1)}

When cultivating magnetotactic bacteria such as MSR-1, it is important to check regularly whether the bacteria remain magnetic. When growing under laboratory conditions, random mutation may lead to a culture of magnetotactic bacteria that has lost the ability to form magnetosomes 239]. In our lab, MSR-1 are grown in $2 \mathrm{~mL}$ tubes. The tubes are closed with a small air head space to ensure a proper reduction in oxygen concentration when the culture grows. Even though this method is simple, its major disadvantage is that we have no information whether the magnetosome formation is as we expect. We cannot open the tubes to take samples, because we will let oxygen in. The better option would be to grow in bioreactors that allow for sampling without disturbing the oxygen concentration, but these are complex and costly and provide quantities that are overkill for lab-on-chip experiments.

The MagOD system offers a solution since we can monitor the growth of MSR-1 bacteria and the magnetosome by keeping cultures in cuvettes inside 


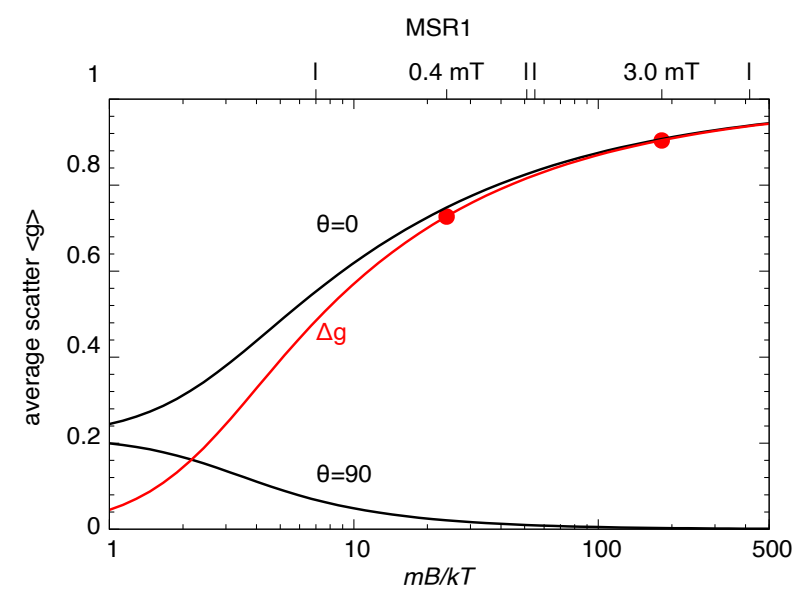

Figure A.13: Calculation of the average scatter factor as a function of the product of the magnetic moment of the magnetsome chain $(m)$ and applied magnetic field $(B)$, for the magnetic field aligned parallel $\left(\theta=0^{\circ}\right)$, and perpendicular $\left(\theta=90^{\circ}\right)$ to the light path (see Figure A.3. The difference in average scatter factor between the orientations is indicated by a red line $(\Delta g)$. From earlier work 234] we estimated that the mean magnetic moment of the magnetosome chain is $0.25 \mathrm{f} \mathrm{Am}^{2}$, with a $10-90 \%$ cutoff of the distribution of 0.07 and $0.57 \mathrm{fAm}^{2}$ respectively. The resulting ranges in $m B / k T$ are indicated on the top axis and by red circles for the mean values of the moments, for the fields used in the experiment of figure A.12

the MagOD meter for long periods. During the growth period we continuously measure the absorbance under change of the external magnetic field. In this way we obtain information on the total number of bacteria, as well as their magnetic response.

We prepared MSR-1 cultures as normal 234], but inserted of tubes we used quartz cuvettes with a PTFE stopper (Hellma QS 110-10-40) to avoid any leaking of oxygen into the cuvette. For the long term observations of figure A.14, the magnetic field was set to loop through cycles of $100 \mathrm{~s}$ consisting of a vertical field of $1.0 \mathrm{mT}(20 \mathrm{~s})$, a horizontal field of $2.9 \mathrm{mT}(20 \mathrm{~s})$ and a vertical field of $0.1 \mathrm{mT}$ $(60 \mathrm{~s})$.

The first transition is at a relatively strong fields, guaranteeing reliable estimations of $C_{\text {mag }}$. The second transition guarantees a relative large time constant which is helpful for accurately estimating $\tau$.

Figure A.14 shows the measured parameters of a sample of MTB over a period of five days. From top to bottom we plot the the optical density (OD), relative $\left(C_{\text {mag }}\right)$ and absolute $\left(\Delta_{\mathrm{OD}}\right)$ magnetic response and relative rotation velocity $(\gamma$, proportional to the ratio between magnetic moment and rotational friction coefficient).

The optical density OD is typical for a bacteria growth sequence. After a lag phase (L), a transition into the exponential growth phase (E) occurs followed by 
the stationary phase $(\mathrm{S})$ where the bacteria concentration remains more or less constant. After three days however, the density increases unexpectedly (X).

During the exponential growth phase, the $C_{\text {mag }}$ decreases sharply. Since $\Delta_{\text {OD }}$ remains constant, we assume that the increase in concentration is entirely due bacteria without magnetosomes. Only after two days we see a gradual increase in magnetic signal (M) due to an increase in the fraction of bacteria with magnetosomes. With the increase in magnetic signal, also $\gamma$ increases, so the magnetic moment of the magnetosome increases compared to the average bacteria length. The observation over the first three days would be consistent with the mechanism that after seeding with magnetic bacteria, growth first proceeds by an increase in non-magnetic bacteria. When that growth stops, the bacteria start to form magnetosomes. This mechanism is in contradiction with electron microscope observations by Stanisland and Yang that magnetosome crystals are equally divided over both parts of the divided cell [240, 241], and will require further experiments.

After approximately 3.3 days a sharp transition occurs (X). As the density of the culture increases again, the magnetic response decreases but the $\gamma$ keeps on increasing. This is a feature we often observe in these measurements. Additionally, we sometimes observe a cloudiness in the suspension, which may be caused by aerotaxis or contamination. Since we do not shake the suspensions before measuring like in a standard optical density meter, these clouds may float in front of the detector and complicate the analysis. It may be possible that rather than rotating individual bacteria, we rotate the entire cloud.

\section{Marathon test: MC-1 velocity measurement}

In contrast to MSR-1 bacteria, that reverse frequently, magnetotactic bacteria of type MC-1 tend to swim for long periods into the same direction 225]. This allows us to collect a large number of bacteria at the bottom of a cuvette, simply by applying a vertical field. After reversing the direction of the field, all bacteria swim upward in a band-shaped cloud. In the MagOD, the passing of this cloud translates to a drop in the photodetector signal. The time between reversal of the field and the response on the photodetector is a measure for the velocity of the bacteria. We nickname this method the 'marathon' test.

To obtain sufficient bacteria for this experiment, we cultivated MC-1 bacteria in a high viscosity agarose based medium in an oxygen gradient, as described by Bazylinski [242], but using low melt agarose instead of bacto agar. The bacteria form a band in the reaction tube, a few $\mathrm{mm}$ below the surface of the medium 243. The easiest way to free the MC-1 from the medium is to pipette a small amount from the band and insert this into a cuvette filled with a low viscosity growth medium from which the agarose has been omitted. The transfer of some agarose cannot be avoided, especially if large quantities of bacteria are desired. 


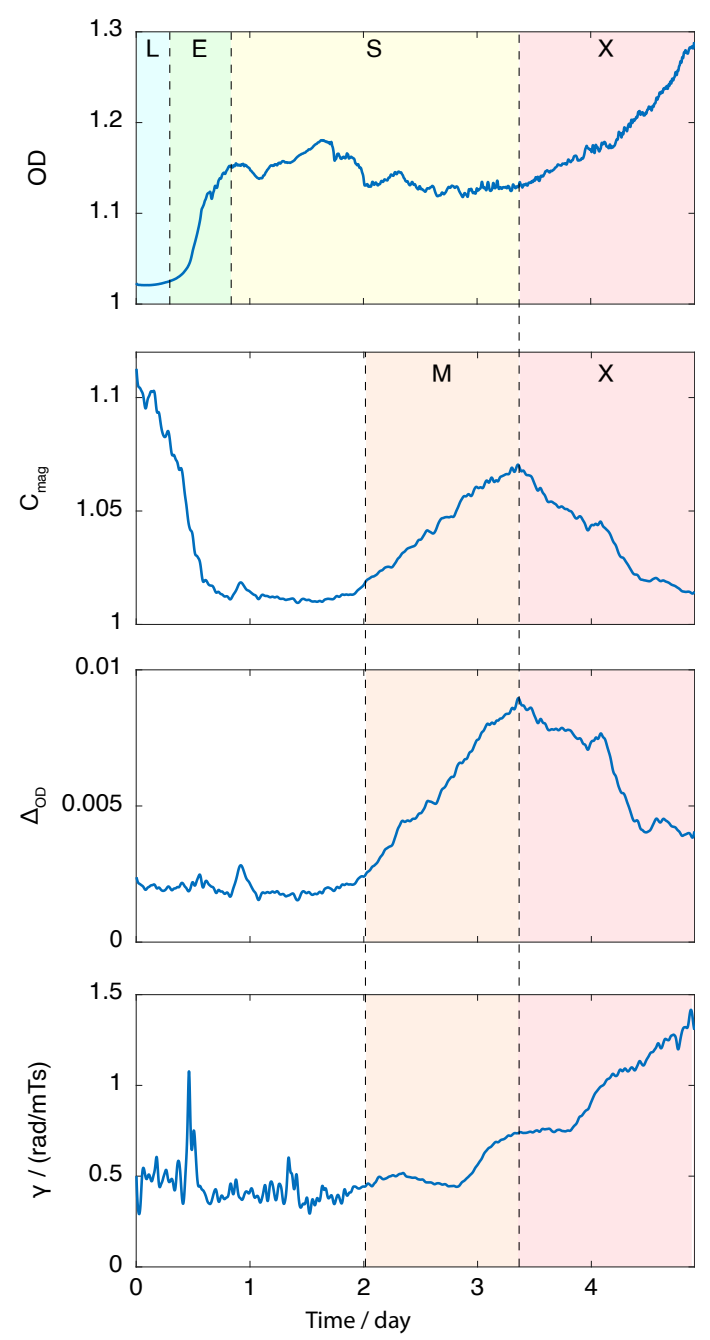

Figure A.14: Four bacteria suspension parameters measured over a span of five days; the optical density (OD), the $C_{\text {mag }}$ (quantifying the ratio of magnetic to nonmagnetic bacteria), the $\Delta_{\mathrm{OD}}$ (quantifying the amount of magnetic bacteria), and the $\gamma$ (quantifying how strong the bacteria respond to magnetic fields). The following phases can be identified: lag phase $(\mathrm{L})$, exponential phase (E), stationary phase (S), magnetic growth phase (M) and an some undefined phase $(\mathrm{X})$. 
Alternatively, one can place a droplet of agarose with bacteria on a side, and position a droplet of growth medium with agarose next to it so that they merge. The MC- 1 can than be directed out of the agarose to the clean droplet with a magnet and collected. This method suffers from a limited amount of bacteria that can be collected and introduction of oxygen is difficult to avoid.

The method we prefer is to pass the mixture of bacteria and agarose through a Pasteur pipette filled with a small plug of cotton. Our assumption is that the cotton breaks up the agarose matrix and perhaps even captures it. By using compressed nitrogen to drive the growth medium with bacteria through the plug, exposure to oxygen can be avoided. To further reduce oxygen exposure, we performed this procedure under nitrogen atmosphere. For this, we simply use a glas beaker with a parafin cover through which the Pasteur pipette is inserted into the cuvette.

Since the MagOD is equipped with a 3D magnetic coil configuration, application of a vertical field, along the cuvette, is simple. A field of $1 \mathrm{mT}$ is applied in the positive $z$-direction for 220 s to collect south seeking bacteria at the bottom of the cuvette. Than the field is reversed so that the collected bacteria swim upwards towards the photodetector. We let the bacteria swim upward for $200 \mathrm{~s}$ and than sequence is repeated again. The asymmetry in time ensures that an sufficient amount of bacteria can assemble at the bottom of the cuvette again. The cloud of bacteria that leaves the bottom of the cuvette disperses due to a distribution of bacteria velocities. To keep the peak sharp and intensity variation high, we reduce the distance between the bottom of the cuvette and the light path to $2.5 \mathrm{~mm}$ by using a special insert.

Figure A.15 shows the output of the photodetector as a function of time since the magnetic field reversal. A series of eight experiments are shown. For each experiment, after approximately $30 \mathrm{~s}$ the cloud reaches the light path, with a maximum density at about $90 \sqrt[6]{6}$ As time progresses, the curves show a similar shape, but at lower amplitude. Apparently, less and less bacteria are collecting at the bottom of the cuvette. The decrease in amplitude, shown in figure A.16 fits very well to an exponential decay $(\exp (-t / \tau))$ with a time constant of approximately half an hour. This suggests that we loose a fixed fraction of bacteria per iteration. The reason for the loss is unclear to us, and would require further investigation.

The arrival time $t$ (s) of MC-1 at the detector fits well to a log-normal distribution (shown as red curves in figure A.15,

$$
f_{\mathrm{t}}(t)=\frac{1}{t \sigma \sqrt{2 \pi}} \exp \left(\frac{-(\ln (t)-\mu)^{2}}{2 \sigma^{2}}\right)(1 / \mathrm{s}),
$$

where $\mu$ (with unit $\ln (s)$ ) and $\sigma$ (unitless) are the mean and standard deviation

\footnotetext{
${ }^{6}$ These experiments are performed with the novel amplifier. Lower intensity results in a higher detector voltage, see figure A.5
} 
of the natural logarithm of $t$. From the distance of the bottom of the cuvette to the light beam $a(2.5 \mathrm{~mm})$ we can calculate the distribution of the velocities $v$ $(\mathrm{m} / \mathrm{s})$,

$$
f_{\mathrm{v}}(v)=\frac{1}{v \sigma \sqrt{2 \pi}} \exp \left(\frac{-\left(\ln \left(\frac{a}{v}\right)-\mu\right)^{2}}{2 \sigma^{2}}\right)(\mathrm{s} / \mathrm{m}) .
$$

The most likely velocity, or mode of this distribution, i: 7

$$
v_{\mathrm{m}}=a \exp \left(-\left(\mu+\sigma^{2}\right)\right)(\mathrm{m} / \mathrm{s}) .
$$

The resulting velocity distributions are shown in figure A.15 in the bottom graph. The curves are offset vertically for clarity, the top curve is the first measurement. The figure shows clearly that the velocity distribution of the bacteria does not change significantly with time. Since the experiment duration was limited to $200 \mathrm{~s}$, the minimum velocity that can be determined is $12.5 \mu \mathrm{m} / \mathrm{s}$. The most likely velocity is on the order of $20 \mu \mathrm{m} / \mathrm{s}$, the fastest bacteria swim approximately $80 \mathrm{\mu m} / \mathrm{s}$.

Figure A.15 is a typical example; we have measured faster as well a slower average velocities. The measured velocity is however considerably lower than that observed by Lefèvre and colleagues by high speed microscopy [219]. From experiments in microfluidic chips, we noted that the velocity distribution is strongly dependent on the duration the MC-1 have been growing in the semisolid medium, temperature (both too high and too low reduce velocity) and oxygen concentation. Further experiments are required to determine the relation between the velocity and these environmental conditions.

\section{A.5 Discussion}

The MagOD magnetic absorbance instrument has proven to be a versatile system that can be successfully applied in the research on magnetotactic bacteria. All designs and source codes are made available, so that the system can be easily replicated, modified and improved. The data presented in this paper may serve as benchmark for future systems. We hope our efforts will inspire colleagues to improve and apply the MagOD in their research. In the following we address possible improvements and suggestions for further research.

\section{A.5.1 Possible improvements}

There are a few issues that will need attention in future iterations of the system, on the level of the measurement head, measurement board and software.

\footnotetext{
${ }^{7}$ Note that the most likely arrival time is $\exp \left(\mu-\sigma^{2}\right)$, so one cannot simply divide the distance travelled by the most likely arrival time to obtain the most likely velocity.
} 

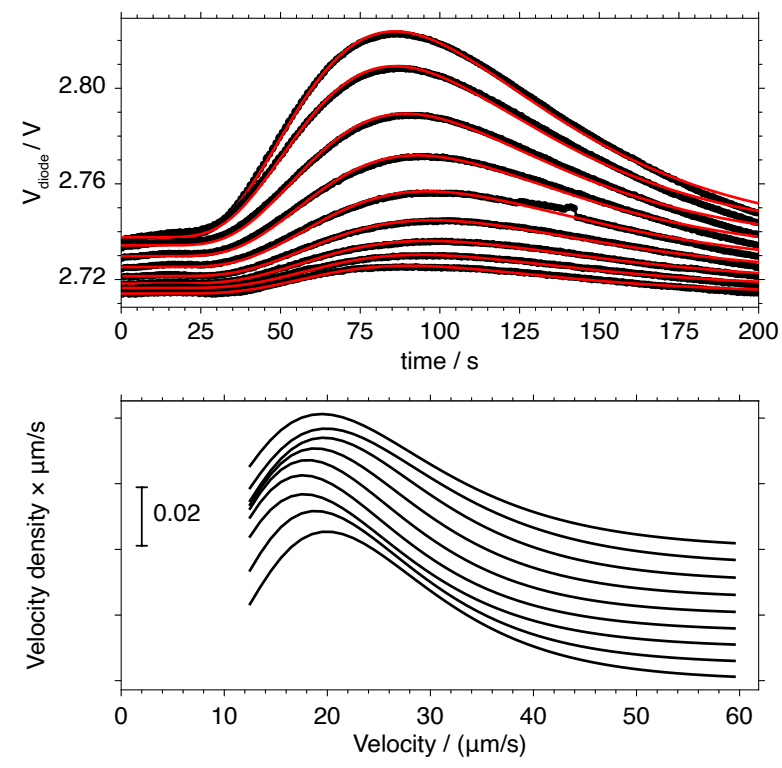

Figure A.15: Output of photodetector as a function of time passed since reversal of the magnetic field. After approximate $30 \mathrm{~s}$, the first MC-1 pass the light beam and scatter the light. Maximum light extinction is reached about $90 \mathrm{~s}$. The experiment is repeated eight times, with intervals of $440 \mathrm{~s}$ between the measurements. The responses fit relatively well to a log-normal distribution (red lines). These fits can be inverted to obtain the velocity distribution of the MC-1 (bottom curve). For clarity, these curves are offset by 0.005 from top to bottom. The velocity distribution remains more or less constant.

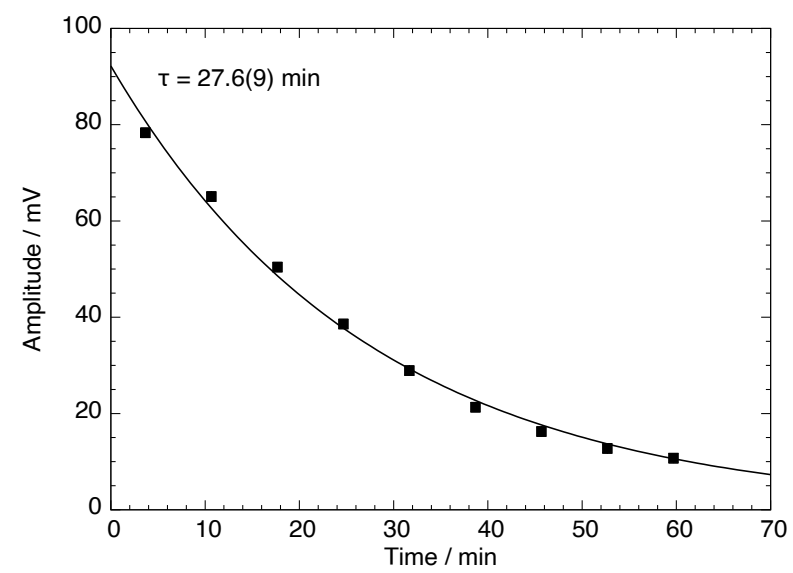

Figure A.16: The decrease in amplitude of the marathon curves of figure A.15 with increasing time fits very well to an exponential decay. 


\section{Measurement head}

The response of the photodiode to the blue LED is non-linear (Figure A.5), for which we do not have a good explanation. Furthermore, the fact that the signal is inversely proportional to the light intensity is counter-intuitive. It may be possible to redesign the amplifier while still maintaining the required footprint. The noise floor in the current design is still a factor of 30 above the theoretical limit, and there are signs that $50 \mathrm{~Hz}$ cross-talk is deteriorating the signal (Figure A.8). One may consider moving the AD converter from the measurement board to the measurement head, so that the signal is better protected from interference. To compensate for drift, automatic offset correction can be applied by modulating the LED intensity periodically.

One may consider moving the drivers for the LEDs to the measurement head, so that the high frequency PWM signal does not have to be transported over the HDMI signal cable and we free up ports on the ESP32. For this, RGB led drivers that communicate over $\mathrm{I}^{2} \mathrm{C}$ are readily available. Care should be taken that the $\mathrm{I}^{2} \mathrm{C}$ clock signal does not interfere with the detection electronics.

In case suspensions with higher densities need to be observed, one may consider solid state lasers that offer at least 100 times higher light intensity.

In contrast to commercial absorbance meters, the MagOD system does not have a piezo actuator to disperse the suspension before measurement. One may consider integrating such a piezo actuator. Alternatively, one may make use of the existing coils and attempt a voice coil actuation principle using a soft magnetic element, additional small coil or even a small permanent magnet mounted in such a way that it does not interfere with the field.

\section{Measurement board}

In future designs of the measurement board, a number of improvements can be made as well. Even though the AD converter is capable of data acquisition at $860 \mathrm{samples} / \mathrm{s}$, we only reach 323 samples/s in practise. We assume this discrepancy is caused by communication overhead that can be optimized.

The current implementation of the current measurement circuits only allows for positive currents. The modification to allow for bi-directional currents is straightforward, for instance by applying an ina266 integrated current monitor.

Finally, the small form factor of micro-SD cards poses a problem in biolab environments since they are easily lost. Removal of the SD card can be avoided if WiFi access is available, but a USB stick may be a better option.

\section{Software}

We expect most development in the software for the MagOD system. Next to improvements in the user interface, currently, the main restriction is that measurement recipes are based on feedforward instructions only (iterations of 
'for this amount of time, set the field and LED color'). There is no capability to react on changes in the detected signal. For instance, it would be very useful if the LED intensity could automatically be adjusted to the absorbance of the suspension under investigation. In marathon experiments, it would be convenient if the field reversal takes place at a fixed delay after occurence of the peak. The current recipe language definition is not capable of handling this type of feedback. We suspect a complete redesign of the software is required, taking full advantage of the EPS32 capabilities. This would be a very interesting task for an (software) engineering student.

\section{A.5.2 Possible future applications}

The four experiments we presented are just some of the possiblities that the novel MagOD systems offers. Without additional modification, we can imagine more experiments, which we report here to inspire future work.

\section{Flagelar motion}

Since the MagOD system has precise field control, it allows for a simple study of the relation between field strength and $C_{\text {mag }}$. It would be interesting to check if the swimming activity of the bacteria themselves contributes to their random motion, which effectively would increase $k T$ and could explain the observed difference. For instance, it would be sufficient to measure $C_{\text {mag }}$ as a function of field before and after killing the MSR-1 (by for example intense UV-light or formaldehyde).

\section{Multi-color OD}

So far we have measured the transmission through MSR-1 cultures only under green light. We notice however that the colour of cultures changes as time passed by. We speculate that these colour changes may be caused by an increase in bacteria size and/or formation of magnetosomes. For long term analysis as in figure A.14, it may therefore be usefull to measure at different wavelengths. In the MagOD system, this can easily achieved by measuring iteratively with the red, green and blue LED. Multiple wavelengths may be combined with the addition of an indicator agent that changes its absorbance spectrum based on changes in conditions.

An example of such an indicator is Resazurin, which reacts to an increase in oxygen concentration with a shift in the absorbance spectrum toward the red. The ratio between the absorbance in the red and green channels could therefore be a measure for the oxygen concentration in the culture, using the blue channel for calibration. 


\section{Modulated light intensity}

The intensity of the LEDs can be varied rapidly, as is illustated in figure A.7. One can use this modulation for differential measurements to correct for intereference signals due to changes in environmental light or cross-talk on the analog signal wiring.

Modulation of the light intensity would also provide information on the photosensitivity of the bacteria. One could for instance measure the $C_{\text {mag }}$ in the red channel before and after a pulse with intense blue light.

\section{Combined marathon and $C_{\text {mag }}$}

We demonstrated $C_{\text {mag }}$ measurements on MSR-1 bacteria and marathon tests on MC-1 bacteria. It is straightforward to combine the marathon test with $C_{\text {mag }}$ measurements. The vertical field ( $z$-direction) should than be switched between zero and for instance a postive value, whereas the field along the light path $(x-$ direction) should be switch between zero and alternatively positive and negative values (So $(x, z)=(0,1),(1,0),(0,1),(-1,0)$, etc). Such an experiment may reveal if the distribution in velocity is related to a distribution in magnetosome strength as well.

\section{Sedimentation}

We often observe an initial increase of light transmission after loading a sample with bacteria. We therefore usually wait until the signal settles. However, there may be information we can extract. We suspect the increase in transmission is caused by sedimentation of debris, such as dead bacteria. If the dead bacteria have magnetosomes, they will still rotate in the magnetic field. So a measurement of $C_{\text {mag }}$ during sedimentation may provide additonal information on the status of the culture.

Moreover, it is very simple to drive only one coil of the vertical coil set. In this way one can generate field gradients that would pull magnetic debris either up or down, thus decelerating or accelerating the sedimentation.

\section{Suspensions of magnetic nanoparticles}

We often try the MagOD system with suspension of magnetic micro- and nanoparticles. This works particularly well for magnetic needles 244] or magnetic discs 245. In principle, spherical particles should not show a change in transmission under rotation of an external field. Magnetic particles however have a tendency to form chains that align with the field (see for instance the work by Yang Gao 246]). Angle and field dependent transmission measurements in the MagOD could therefore provide information on the dynamic interaction 
between particles. Perhaps one can extend of the use of the MagOD beyond the magnetotactic research community.

\section{A.6 Conclusion}

We constructed a magnetic spectrophotometer (magnetic optical density meter, or MagOD), which analyses the amount of light transmitted through a suspension of a magnetotactic bacteria in a transparent cuvette under application of a magnetic field.

Light transmission measurements with the novel MagOD system were compared with a commercial instrument (Eppendorf BioPhotoMeter), using of a dilution series of a magnetic nanoparticle suspension. The deviation between the new OD meter and the commercial instrument is below 0.2 in terms of relative absorbance for wavelengths ranging from 460 to $645 \mathrm{~nm}$. The blue channel however suffers from non-linearity and should only be used at low intensity. The dynamic range (from noise level to maximum signal) of the new system is $88 \mathrm{~dB}$ (OD of 4.4), where the commercial system reaches $53 \mathrm{~dB}$ (OD of 2.6). The MagOD system is considerably faster, with a sample rate of 323 samples/s. The commercial instrument has a sampling time in excess of $1 \mathrm{~s}$.

The magnetic field can be applied in three directions, with a setpoint resolution of $70 \mathrm{nT}$ and a ripple of less than $50 \mu \mathrm{T}$. The maximum field is $5.1(1) \mathrm{mT}$, but limited in duration due to coil heating. When a field of $1.0 \mathrm{mT}$ is continuously applied, the temperature increase of cuvette is approximately $1 \mathrm{~K} / \mathrm{h}$ and limited to $2.1(3) \mathrm{K}$.

The MagOD system was used to characterize various aspects of MSR-1 and MC-1 magnetotactic bacteria.

By means of the magnetic field, MSR-1 were oriented at different angles with respect to the light path. The transmission is high when bacteria are aligned along the light beam and reduces when the bacteria are aligned perpendicular to the light path. The relation between angle and optical density can be approximated relatively well by a sine.

From the difference in transmission, we can derive a measure for the amount of magnetic bacteria. This fraction is commonly expressed as a ratio $\left(C_{\text {mag }}\right)$, which is a parameter that increases with the relative fraction of magnetic bacteria compared to the total number of bacteria. It can also be expressed as a difference $\left(\Delta_{\mathrm{OD}}\right)$, which is a measure for the absolute amount of magnetic bacteria. Both parameters increase with applied field strength in a way that is within measurement error in agreement with a simple model based on Brownian motion.

We used the MagOD system to continuously monitor the development of a culture of MSR-1 magnetotactic bacteria over 5 days. We recorded the optical density $\left(O_{\mathrm{D}}\right)$, change in light transmission under rotation of the magnetic field 
$\left(C_{\mathrm{mag}}\right.$ and $\left.\Delta_{\mathrm{OD}}\right)$ and the rotation velocity of the bacteria $(\gamma)$. We could clearly distinguish separate growth phases (lag, exponential growth, stationary). The increase in magnetic response $\left(C_{\mathrm{mag}}\right.$ and $\left.\Delta_{\mathrm{OD}}\right)$ takes place during the stationary phase.

Unipolar bacteria, such as MC-1, can be collected at the bottom of the cuvette with a vertical magnetic field. Upon reversal of the field the entire group departs from the bottom and will arrive at the light beam, causing a dip in the transmitted light. This 'marathon' test allows us to measure the velocity distribution.

The arrival times can be accurately described by a lognormal distribution, with a mode (most occurring velocity) of $20 \mu \mathrm{m} / \mathrm{s}$. The maximum velocity observed is in the order of $80 \mu \mathrm{m} / \mathrm{s}$. The amount of bacteria participating in the marathon test decreases exponentially with each test with a time constant of approximately half an hour.

The dedicated magnetic optical density meter presented here is relatively simple and inexpensive, yet the data that can be extracted from magnetotactic bacteria cultures is rich in detail. All information for the construction of the device, including 3D print designs, printed circuit board layouts and code for the microprocessor has been made available online. The authors trust that the magnetotactic bacteria community will benefit from our work, and that the MagOD instrument will become a valuable tool for research on magnetotactic bacteria.

\section{A.7 $C_{\text {mag }}^{*}$ and $C_{\text {mag }}$ approximations}

The effect of a rotation of the magnetic field is usually small. It therefore is useful to express the variation with respect to the average intensity or absorbance 8

$$
\begin{array}{r}
I_{\mathrm{s}}=\frac{I(0)+I(90)}{2}, \\
A=\log \left(I_{\mathrm{ref}} / I_{\mathrm{s}}\right) .
\end{array}
$$

by a small deviation $\alpha$

$$
\begin{aligned}
A(0) & =(1+\alpha) A \\
A(90) & =(1-\alpha) A
\end{aligned}
$$

so that

$$
\Delta_{\mathrm{A}}=2 \alpha A
$$

\footnotetext{
${ }^{8} A=O_{\mathrm{D}}$
} 
and

$$
C_{\mathrm{mag}}=\frac{(1+\alpha)}{(1-\alpha)} \approx 1+2 \alpha=1+\frac{\Delta_{\mathrm{A}}}{A} .
$$

The approximation is better than $5 \%$ in terms of $C_{\text {mag }}-1$ for $C_{\text {mag }}<1.1$.

Similarly, we can define for the estimate of $C_{\mathrm{mag}}^{*}$

$$
\Delta I=2 \beta I_{\mathrm{s}}
$$

so that

$$
C_{\mathrm{mag}}^{*} \approx 1+\frac{I_{\mathrm{s}}}{I_{\mathrm{ref}}-I_{\mathrm{s}}} 2 \beta=1+\frac{\Delta I}{I_{\mathrm{ref}}-I_{\mathrm{s}}}
$$

Both definitions of $C_{\text {mag }}$ can be related by realizing that

$$
\frac{I(0)}{I_{\mathrm{ref}}}=\left(\frac{I_{\mathrm{s}}}{I_{\mathrm{ref}}}\right)^{1+\alpha} \approx \frac{I_{\mathrm{s}}}{I_{\mathrm{ref}}}\left(1+\alpha \ln \left(I_{\mathrm{s}} / I_{\mathrm{ref}}\right)\right)
$$

and similarly for $I(90)$ with $-\alpha$, so that

$$
\Delta I=-2 \alpha I_{\mathrm{s}} \ln \left(I_{\mathrm{s}} / I_{\text {ref }}\right)
$$

So in the approximation for $C_{\text {mag }}$ close to unity, the relation between both definitions is

$$
\begin{gathered}
\frac{C_{\mathrm{mag}}-1}{C_{\mathrm{mag}}^{*}-1} \approx \frac{\Delta_{\mathrm{A}}}{A} \frac{I_{\mathrm{ref}}-I_{\mathrm{s}}}{\Delta I}= \\
\frac{I_{\mathrm{ref}}-I_{\mathrm{s}}}{I_{\mathrm{s}} \ln \left(I_{\mathrm{ref}} / I_{\mathrm{s}}\right)}=\frac{\left(I_{\mathrm{ref}}-I_{s}\right) \log (e)}{I_{\mathrm{s}} A} .
\end{gathered}
$$

The definitions converge for $I_{\mathrm{s}} \rightarrow I_{\text {ref }}$, so for samples with very low optical density.

\section{A.8 Cotangens approximation}

For fitting purposes, the rather complicated cotangens expression of equation A.5 can be approximated by a much simpler exponential function. The fit was performed in gnuplot, resulting in a fit parameter $0.85(1)$. The error is less than $0.065 \mathrm{rad}$, as shown in figure A.17 


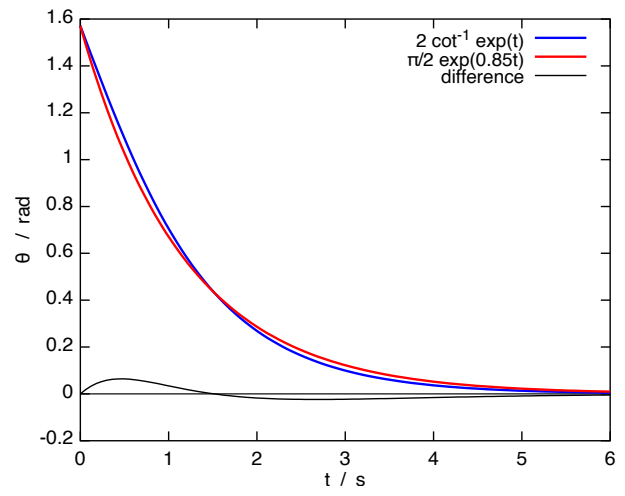

Figure A.17: Approximation by an exponential function of the exact solution to the differential equation for the rotation of the bacterium as a function of time (equation A.5. The optimal fit is for a prefactor $0.85(1)$, in which case the error is smaller than $0.065 \mathrm{rad}$.

\section{A.9 Measurements}

Figure A.18 shows the projection on a white paper sheet of the light exiting from the measurement head (with the photodetector circuit board removed). The pictures are snapshots of a video taken with an iPhone camera for a range in LED duty cycles (measurement of figure A.19). The full video is available in the Supplementary Material (MagODLEDprojection.mov). The opening behind the cuvette is a square hole of $3 \mathrm{~mm} \times 3 \mathrm{~mm}$, which is clearly visible. The photodetector itself has an area of $2.7 \mathrm{~mm} \times 2.7 \mathrm{~mm}$, so is collecting the inner fraction of this pattern. For the green and blue led, some echo images can be observed. The three patterns do not align, which is most likely caused by the fact that the three LED in the WP154 housing are not centered to the axis of the front lens. From the distance between the projected image and the LED (approximately $15 \mathrm{~cm}$ ) and the maximum shift of about $5 \mathrm{~mm}$ we estimate that the misalignment is in the order of $2^{\circ}$. Since the photodetector is mounted directly behind the opening behind the cuvette, this misalignment is of no consequence.

Figure A.19 shows the intensity on the photodetector as a function of the duty cycle of each of the three LEDs, for a cuvette filled with water and three different dilutions of a EMG304 magnetic nanoparticle suspension. From the difference in slopes the absorbance relative to the water filled cuvette is determined. This measurement is used for the MagOD datapoints in figure A.6. The blue LED suffers from artefacts. The slope is not constant, but reduce at high intensity. Furthermore, there is a small step at an intensity of about 0.6. For the estimate of absorbance of the blue LED, only the linear region at low intensity was used. 


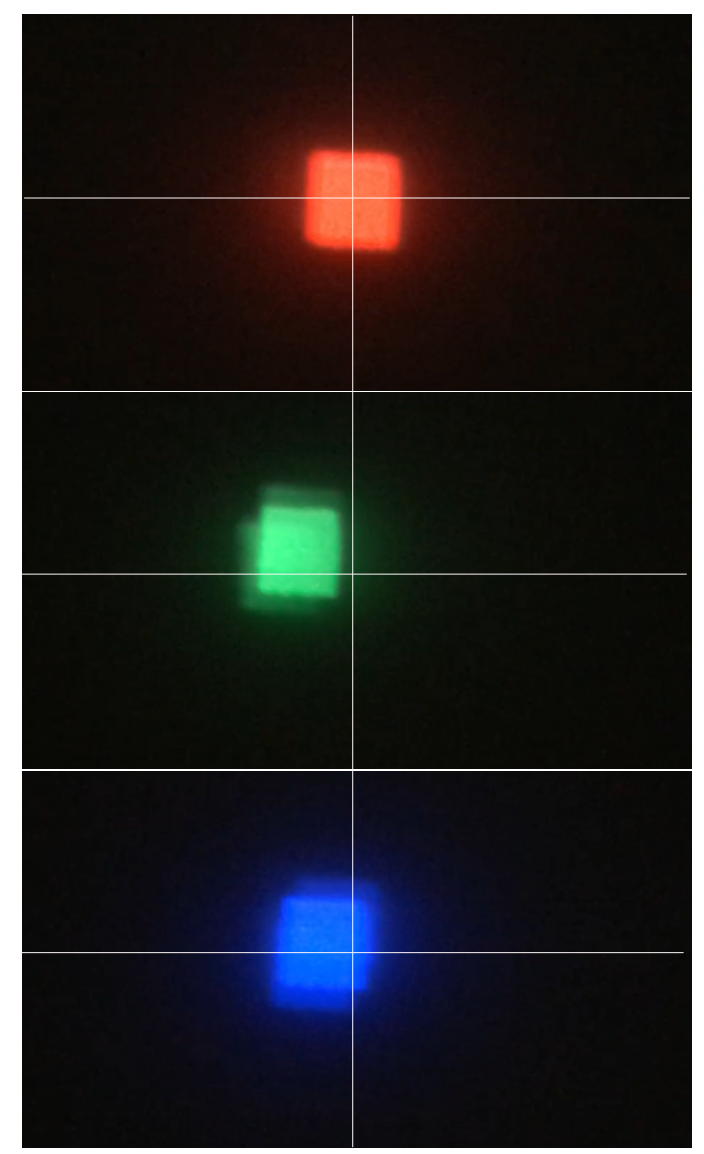

Figure A.18: Projection of the light pattern of the three different LEDs. The $3 \mathrm{~mm} \times 3 \mathrm{~mm}$ opening at the back of the cuvette can clearly be seen. The green and blue led show some reflection, and the patterns are not aligned. The estimated misalignment is in the order of $2^{\circ}$. Since the photodetector has a sensitive area of $2.7 \mathrm{~mm} \times 2.7 \mathrm{~mm}$ and is directly mounted behind the opening in the holder, we do not expect any adverse effects from the reflections of misalignment. A full video of the pattern shape as function of the intensity is available in the supplementary material (Supplementary Material ). 


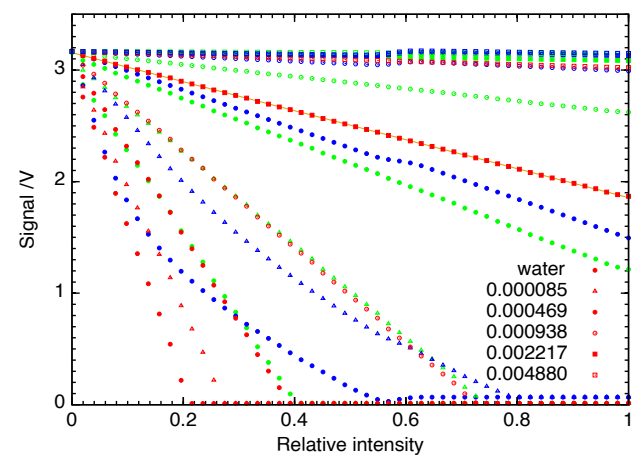

Figure A.19: Signal as a function of the duty cycle of the LED, for the three different LED wavelengths and for a water reference and three different dilutions of a EMG304 magnetic nanoparticle suspension. 


\section{References}

[1] B. Siciliano and O. Khatib, Springer Handbook of Robotics, B. Siciliano and O. Khatib, Eds. Cham: Springer International Publishing, 2016, pp. 1-2227, ISBN: 978-3-319-325507. DOI: $10.1007 / 978-3-319-32552-1$

[2] "Presentation World Robotics by the International Federation of Robotics." (2020), [Online]. Available: https://ifr.org/downloads/press2018/Presentation\%7B\%5C_ \%7DWR\%7B\%5C_\%7D2020.pdf (visited on 12/14/2020).

[3] "Presentation Service Robots by the International Federation of Robotics." (2020), [Online]. Available: https://ifr.org/downloads/press2018/Presentation\%7B\%5C_ $\%$ 7DService\%7B\%5C_\%7DRobots\%7B\%5C_\%7D2020\%7B\%5C_\%7DReport . pdf (visited on $12 / 14 / 2020)$.

[4] P. Cooke, "Gigafactory Logistics in Space and Time: Tesla's Fourth Gigafactory and Its Rivals," Sustainability, vol. 12, no. 5, p. 2044, Mar. 2020. DOI: 10.3390/su12052044

[5] Y. Gao and S. Chien, "Review on space robotics: Toward top-level science through space exploration," Science Robotics, vol. 2, no. 7, eaan5074, Jun. 2017. DOI: 10.1126/ scirobotics.aan5074

[6] B. Arad, J. Balendonck, R. Barth, O. Ben-Shahar, Y. Edan, T. Hellström, J. Hemming, P. Kurtser, O. Ringdahl, T. Tielen, and B. Tuijl, "Development of a sweet pepper harvesting robot," Journal of Field Robotics, vol. 37, no. 6, pp. 1027-1039, Sep. 2020. DOI: $10.1002 /$ rob.21937.

[7] T. Detert, S. Charaf Eddine, J.-C. Fauroux, T. Haschke, F. Becchi, B. Corves, R. Guzman, F. Herb, B. Linéatte, and D. Martin, "Bots2ReC: introducing mobile robotic units on construction sites for asbestos rehabilitation," Construction Robotics, vol. 1, no. 1-4, pp. 29-37, Dec. 2017. DOI: 10.1007/s41693-017-0007-1

[8] I. Tsitsimpelis, C. J. Taylor, B. Lennox, and M. J. Joyce, "A review of ground-based robotic systems for the characterization of nuclear environments," Progress in Nuclear Energy, vol. 111, no. May 2018, pp. 109-124, Mar. 2019. DoI: 10.1016/j.pnucene.2018 10.023

[9] L. Lopes, N. Zajzon, B. Bodo, S. Henley, G. Žibret, and T. Dizdarevic, "UNEXMIN: developing an autonomous underwater explorer for flooded mines," Energy Procedia, vol. 125, pp. 41-49, Sep. 2017. DOI: 10.1016/j.egypro.2017.08.051

[10] L. Marconi, C. Melchiorri, M. Beetz, D. Pangercic, R. Siegwart, S. Leutenegger, R. Carloni, S. Stramigioli, H. Bruyninckx, P. Doherty, A. Kleiner, V. Lippiello, A. Finzi, B. Siciliano, A. Sala, and N. Tomatis, "The SHERPA project: Smart collaboration between humans and ground-aerial robots for improving rescuing activities in alpine environments," in International Symposium on Safety, Security, and Rescue Robotics (SSRR), IEEE Robotics and Automation Society, Nov. 2012, pp. 1-4, ISBN: 978-1-47990165-4. DOI: $10.1109 /$ SSRR.2012.6523905.

[11] R. L. Finn and D. Wright, "Unmanned aircraft systems: Surveillance, ethics and privacy in civil applications," Computer Law \& Security Review, vol. 28, no. 2, pp. 184-194, Apr. 2012. DOI: $10.1016 / \mathrm{j} . \mathrm{cls} .2012 .01 .005$

[12] E. R. Teoh and D. G. Kidd, "Rage against the machine? Google's self-driving cars versus human drivers," Journal of Safety Research, vol. 63, pp. 57-60, Dec. 2017. DOI: $10.1016 / j \cdot j$ sr.2017.08.008 
[13] J. Jones, "Robots at the tipping point: the road to iRobot Roomba," IEEE Robotics E Automation Magazine, vol. 13, no. 1, pp. 76-78, Mar. 2006. DOI: 10.1109/MRA. 2006 1598056 arXiv: $\mathbf{z} 0022$

[14] V. Groenhuis, M. Chandrapal, S. Stramigioli, and X. Chen, "Controlling pneumatic artificial muscles in exoskeletons with surface electromyography," 14th Mechatronics Forum International Conference, MECHATRONICS 2014, pp. 451-457, 2014.

[15] S. DiMaio, M. Hanuschik, and U. Kreaden, "The da Vinci Surgical System," in Surgical Robotics, J. Rosen, B. Hannaford, and R. M. Satava, Eds., Boston, MA: Springer US, 2011, pp. 199-217, ISBN: 978-1-4419-1126-1. DOI: 10.1007/978-1-4419-1126-1_9

[16] C. Causer, "Disney tech: Immersive storytelling through innovation," IEEE Potentials, vol. 38, no. 5, pp. 10-18, Aug. 2019. DOI: 10.1109/MPOT.2019.2919851

[17] C. Freschi, V. Ferrari, F. Melfi, M. Ferrari, F. Mosca, and A. Cuschieri, "Technical review of the da Vinci surgical telemanipulator," The International Journal of Medical Robotics and Computer Assisted Surgery, vol. 9, no. 4, pp. 396-406, Dec. 2013. DOI: 10.1002/rcs.1468.

[18] D. B. Camarillo, T. M. Krummel, and J. K. Salisbury, "Robotic technology in surgery: Past, present, and future," American Journal of Surgery, vol. 188, no. 4 SUPPL. 1, pp. 2-15, 2004. DOI: 10.1016/j.amjsurg.2004.08.025

[19] G. Z. Yang, J. Cambias, K. Cleary, E. Daimler, J. Drake, P. E. Dupont, N. Hata, P. Kazanzides, S. Martel, R. V. Patel, V. J. Santos, and R. H. Taylor, "Medical roboticsRegulatory, ethical, and legal considerations for increasing levels of autonomy," Science Robotics, vol. 2, no. 4, pp. 1-2, 2017. DOI: 10.1126/scirobotics.aam8638

[20] J. Troccaz, G. Dagnino, and G. Z. Yang, "Frontiers of Medical Robotics: From Concept to Systems to Clinical Translation," Annual Review of Biomedical Engineering, vol. 21, pp. 193-218, 2019. DOI: 10.1146/annurev-bioeng-060418-052502

[21] J. Troccaz, M. Peshkin, and B. Davies, "Medical Robotic Systems in ComputerIntegrated Surgery," in CVRMed-MRCAS'97, J. Troccaz, E. Grimson, and R. Mösges, Eds., Springer Berlin Heidelberg, Jun. 1997, pp. 725-736. DOI: $10.1097 / 01$.sgs $0000081179.89384 . \mathrm{ba}$

[22] M. Caversaccio, W. Wimmer, J. Anso, G. Mantokoudis, N. Gerber, C. Rathgeb, D. Schneider, J. Hermann, F. Wagner, O. Scheidegger, M. Huth, L. Anschuetz, M. Kompis, T. Williamson, B. Bell, K. Gavaghan, and S. Weber, "Robotic middle ear access for cochlear implantation: First in man," PLoS ONE, vol. 14, no. 8, pp. 1-12, 2019. DOI: 10.1371/journal.pone.0220543

[23] O. Boubaker, Medical robotics. Elsevier Inc., 2020, pp. 153-204, ISBN: 9780128213506. DOI: $10.1016 / \mathrm{b} 978-0-12-821350-6.00007-\mathrm{x}$

[24] F. J. Siepel, B. Maris, M. K. Welleweerd, V. Groenhuis, P. Fiorini, and S. Stramigioli, "Needle and Biopsy Robots: a Review," Current Robotics Reports, vol. 2, no. 1, pp. 7384, Mar. 2021. DOI: 10.1007/s43154-020-00042-1.

[25] R. Taylor, "A Perspective on Medical Robotics," Proceedings of the IEEE, vol. 94, no. 9, pp. 1652-1664, Sep. 2006. DOI: 10.1109/JPROC.2006.880669

[26] H. Sung, J. Ferlay, R. L. Siegel, M. Laversanne, I. Soerjomataram, A. Jemal, and F. Bray, "Global Cancer Statistics 2020: GLOBOCAN Estimates of Incidence and Mortality Worldwide for 36 Cancers in 185 Countries," CA: A Cancer Journal for Clinicians, vol. 71, no. 3, pp. 209-249, May 2021. DOI: 10.3322/caac.21660

[27] H.-P. Sinn and H. Kreipe, "A Brief Overview of the WHO Classification of Breast Tumors, 4th Edition, Focusing on Issues and Updates from the 3rd Edition," Breast Care, vol. 8, no. 2, pp. 149-154, 2013. Dor: 10.1159/000350774 
[28] F. Bray, J. Ferlay, I. Soerjomataram, R. L. Siegel, L. A. Torre, and A. Jemal, "Global cancer statistics 2018: GLOBOCAN estimates of incidence and mortality worldwide for 36 cancers in 185 countries," CA: A Cancer Journal for Clinicians, vol. 68, no. 6, pp. 394-424, Nov. 2018. DOI: 10.3322/caac. 21492

[29] C. H. Lee, D. D. Dershaw, D. Kopans, P. Evans, B. Monsees, D. Monticciolo, R. J. Brenner, L. Bassett, W. Berg, S. Feig, E. Hendrick, E. Mendelson, C. D'Orsi, E. Sickles, and L. W. Burhenne, "Breast Cancer Screening With Imaging: Recommendations From the Society of Breast Imaging and the ACR on the Use of Mammography, Breast MRI, Breast Ultrasound, and Other Technologies for the Detection of Clinically Occult Breast Cancer," Journal of the American College of Radiology, vol. 7, no. 1, pp. 18-27, 2010. DOI: $10.1016 / \mathrm{j} \cdot \mathrm{jacr} .2009 .09 .022$

[30] E. Altobelli, L. Rapacchietta, P. Angeletti, L. Barbante, F. Profeta, and R. Fagnano, "Breast Cancer Screening Programmes across the WHO European Region: Differences among Countries Based on National Income Level," International Journal of Environmental Research and Public Health, vol. 14, no. 4, p. 452, Apr. 2017. DOI: 10.3390/ijerph14040452

[31] R. J. Bleicher and M. Morrow, "MRI and breast cancer: role in detection, diagnosis, and staging," Oncology, vol. 21, no. 12, pp. 1521-1528, Nov. 2007.

[32] K. M. Kelly, J. Dean, W. S. Comulada, and S.-J. Lee, "Breast cancer detection using automated whole breast ultrasound and mammography in radiographically dense breasts," European Radiology, vol. 20, no. 3, pp. 734-742, Mar. 2010. DOI: 10.1007/s00330-009-1588-y

[33] C. R. Saccarelli, A. G. V. Bitencourt, and E. A. Morris, "Breast Cancer Screening in High-Risk Women: Is MRI Alone Enough?" JNCI: Journal of the National Cancer Institute, vol. 112, no. 2, pp. 121-122, Feb. 2020. DOI: 10.1093/jnci/djz130.

[34] C. K. Kuhl, S. Schrading, C. C. Leutner, N. Morakkabati-Spitz, E. Wardelmann, R. Fimmers, W. Kuhn, and H. H. Schild, "Mammography, Breast Ultrasound, and Magnetic Resonance Imaging for Surveillance of Women at High Familial Risk for Breast Cancer," Journal of Clinical Oncology, vol. 23, no. 33, pp. 8469-8476, Nov. 2005. DOI: $10.1200 / \mathrm{JCO} .2004 .00 .4960$

[35] A. S. Tagliafico, M. Piana, D. Schenone, R. Lai, A. M. Massone, and N. Houssami, "Overview of radiomics in breast cancer diagnosis and prognostication," The Breast, vol. 49, pp. 74-80, Feb. 2020. DOI: 10.1016/j.breast.2019.10.018

[36] Q. Hu, H. M. Whitney, and M. L. Giger, "A deep learning methodology for improved breast cancer diagnosis using multiparametric MRI," Scientific Reports, vol. 10, no. 1, pp. 1-11, 2020. DOI: 10.1038/s41598-020-67441-4

[37] U. Bick, R. M. Trimboli, A. Athanasiou, C. Balleyguier, P. A. T. Baltzer, M. Bernathova, K. Borbély, B. Brkljacic, L. A. Carbonaro, P. Clauser, E. Cassano, C. Colin, G. Esen, A. Evans, E. M. Fallenberg, M. H. Fuchsjaeger, F. J. Gilbert, T. H. Helbich, S. H. Heywang-Köbrunner, M. Herranz, K. Kinkel, F. Kilburn-Toppin, C. K. Kuhl, M. Lesaru, M. B. I. Lobbes, R. M. Mann, L. Martincich, P. Panizza, F. Pediconi, R. M. Pijnappel, K. Pinker, S. Schiaffino, T. Sella, I. Thomassin-Naggara, A. Tardivon, C. V. Ongeval, M. G. Wallis, S. Zackrisson, G. Forrai, J. C. Herrero, and F. Sardanelli, "Image-guided breast biopsy and localisation: recommendations for information to women and referring physicians by the European Society of Breast Imaging," Insights into Imaging, vol. 11, no. 1, p. 12 , Dec. 2020. DOI: 10.1186/s13244-019-0803-x

[38] D. M. Plecha, C. Garlick, C. Dubchuck, C. Thompson, and N. Constantinou, "Comparing cancer detection rates of patients undergoing short term follow-up vs routine follow-up after benign breast biopsies, is follow-up needed?" Clinical Imaging, vol. 42, pp. 37-42, Mar. 2017. DOI: 10.1016/j.clinimag.2016.11.007. 
[39] G. Suman and A. Patra, "Current Imaging Techniques in Breast Cancer: An Overview," in Current Advances in Breast Cancer Research: A Molecular Approach, Bentham Science Publishers, Apr. 2020, ch. 2, pp. 30-60, ISBN: 978-1681087719. DOI: 10.2174/ 9789811451447120010005 .

[40] J. Y. Zhou, J. Tang, Z. L. Wang, F. Q. Lv, Y. K. Luo, H. Z. Qin, and M. Liu, “Accuracy of 16/18G core needle biopsy for ultrasound-visible breast lesions," World Journal of Surgical Oncology, vol. 12, no. 1, pp. 1-7, 2014. DOI: 10.1186/1477-7819-12-7

[41] G. Schueller, C. Schueller-Weidekamm, and T. H. Helbich, "Accuracy of ultrasoundguided, large-core needle breast biopsy," European Radiology, vol. 18, no. 9, pp. 17611773, Sep. 2008. DOI: 10.1007/s00330-008-0955-4

[42] S. Chikarmane, B. Jin, and C. Giess, "Accuracy of MRI-directed ultrasound and subsequent ultrasound-guided biopsy for suspicious breast MRI findings," Clinical Radiology, vol. 75, no. 3, pp. 185-193, Mar. 2020. DOI: 10.1016/j.crad.2019.10.013.

[43] C. Meeuwis, J. Veltman, H. N. van Hall, R. D. M. Mus, C. Boetes, J. O. Barentsz, and R. M. Mann, "MR-guided breast biopsy at 3T: diagnostic yield of large core needle biopsy compared with vacuum-assisted biopsy," European Radiology, vol. 22, no. 2, pp. 341-349, Feb. 2012. DOI: 10.1007/s00330-011-2272-6

[44] V. Y. Park, M. J. Kim, E.-K. Kim, and H. J. Moon, "Second-Look US: How to Find Breast Lesions with a Suspicious MR Imaging Appearance," RadioGraphics, vol. 33, no. 5, pp. 1361-1375, Sep. 2013. DOI: $10.1148 /$ rg.335125109

[45] A. L. McGrath, E. R. Price, P. R. Eby, and H. Rahbar, "MRI-guided breast interventions," Journal of Magnetic Resonance Imaging, vol. 46, no. 3, pp. 631-645, Sep. 2017. DOI: $10.1002 / \mathrm{jmri.25738}$

[46] R. H. El Khouli, K. J. Macura, P. B. Barker, L. M. Elkady, M. A. Jacobs, J. VogelClaussen, and D. A. Bluemke, "MRI-guided vacuum-assisted breast biopsy: A phantom and patient evaluation of targeting accuracy," Journal of Magnetic Resonance Imaging vol. 30, no. 2, pp. 424-429, Aug. 2009. DOI: 10.1002/jmri.21831 arXiv: NIHMS150003

[47] A. G. Waks and E. P. Winer, "Breast Cancer Treatment," Journal of the American Medical Association, vol. 321, no. 3, p. 288, Jan. 2019. DOI: 10.1001/jama.2018.19323

[48] K. You, S. Park, J. M. Ryu, I. Kim, S. K. Lee, J. Yu, S. W. Kim, S. J. Nam, and J. E. Lee, "Comparison of core needle biopsy and surgical specimens in determining intrinsic biological subtypes of breast cancer with immunohistochemistry," Journal of Breast Cancer, vol. 20, no. 3, pp. 297-303, 2017. DOI: 10.4048/jbc.2017.20.3.297

[49] J. H. Youk, E.-K. Kim, M. J. Kim, J. Y. Lee, and K. K. Oh, "Missed Breast Cancers at US-guided Core Needle Biopsy: How to Reduce Them," RadioGraphics, vol. 27, no. 1, pp. 79-94, Jan. 2007. DOI: $10.1148 /$ rg. 271065029

[50] T. Vajsbaher, H. Schultheis, and N. K. Francis, "Spatial cognition in minimally invasive surgery: a systematic review," BMC Surgery, vol. 18, no. 1, p. 94, Dec. 2018. DOI: 10.1186/s12893-018-0416-1

[51] E. Y. Chae, H. J. Shin, H. J. Kim, H. Yoo, S. Baek, J. H. Cha, and H. H. Kim, "Diagnostic Performance of Automated Breast Ultrasound as a Replacement for a Hand-Held Second-Look Ultrasound for Breast Lesions Detected Initially on Magnetic Resonance Imaging," Ultrasound in Medicine \& Biology, vol. 39, no. 12, pp. 2246-2254, Dec. 2013. DOI: 10.1016/j.ultrasmedbio.2013.07.005

[52] Y. Kim, B. J. Kang, S. H. Kim, and E. J. Lee, "Prospective Study Comparing Two Second-Look Ultrasound Techniques," Journal of Ultrasound in Medicine, vol. 35, no. 10, pp. 2103-2112, Oct. 2016. DoI: 10.7863/ultra.15.11076 
[53] R. Girometti, M. Zanotel, V. Londero, M. Bazzocchi, and C. Zuiani, "Comparison between automated breast volume scanner (ABVS) versus hand-held ultrasound as a second look procedure after magnetic resonance imaging," European Radiology, vol. 27, no. 9, pp. 3767-3775, Sep. 2017. DOI: 10.1007/s00330-017-4749-4

[54] A. Fausto, M. Bernini, D. La Forgia, A. Fanizzi, M. Marcasciano, L. Volterrani, D. Casella, and M. A. Mazzei, "Six-year prospective evaluation of second-look US with volume navigation for MRI-detected additional breast lesions," European Radiology, vol. 29, no. 4, pp. 1799-1808, Apr. 2019. DOI: 10.1007/s00330-018-5765-8

[55] N. Duric, P. Littrup, C. Li, O. Roy, S. Schmidt, R. Janer, X. Cheng, J. Goll, O. Rama, L. Bey-Knight, and W. Greenway, "Breast ultrasound tomography: bridging the gap to clinical practice," in Medical Imaging 2012: Ultrasonic Imaging, Tomography, and Therapy, J. G. Bosch and M. M. Doyley, Eds., vol. 8320, Feb. 2012, 83200O, ISBN: 9780819489692. DOI: $10.1117 / 12.910988$

[56] D. Amy, Lobar Approach to Breast Ultrasound, D. Amy, Ed. Cham: Springer International Publishing, 2018, pp. 325-335, ISBN: 978-3-319-61680-3. DOI: 10.1007/978-3319-61681-0

[57] C. Schmachtenberg, T. Fischer, B. Hamm, and U. Bick, "Diagnostic Performance of Automated Breast Volume Scanning (ABVS) Compared to Handheld Ultrasonography With Breast MRI as the Gold Standard," Academic Radiology, vol. 24, no. 8, pp. 954961, Aug. 2017. DOI: 10.1016/j.acra.2017.01.021

[58] S. Nakano, M. Yoshida, K. Fujii, K. Yorozuya, J. Kousaka, Y. Mouri, T. Fukutomi, Y. Ohshima, J. Kimura, and T. Ishiguchi, "Real-Time Virtual Sonography, A Coordinated Sonography and MRI System that Uses Magnetic Navigation, Improves the Sonographic Identification of Enhancing Lesions on Breast MRI," Ultrasound in Medicine \& Biology, vol. 38, no. 1, pp. 42-49, Jan. 2012. DOI: 10.1016/j.ultrasmedbio.2011.10.005

[59] S. Nakano, J. Kousaka, K. Fujii, K. Yorozuya, M. Yoshida, Y. Mouri, M. Akizuki, R. Tetsuka, T. Ando, T. Fukutomi, Y. Oshima, J. Kimura, T. Ishiguchi, and O. Arai, "Impact of real-time virtual sonography, a coordinated sonography and MRI system that uses an image fusion technique, on the sonographic evaluation of MRI-detected lesions of the breast in second-look sonography," Breast Cancer Research and Treatment, vol. 134, no. 3, pp. 1179-1188, Aug. 2012. DOI: 10.1007/s10549-012-2163-9.

[60] A. Y. Park and B. K. Seo, "Real-Time MRI Navigated Ultrasound for Preoperative Tumor Evaluation in Breast Cancer Patients: Technique and Clinical Implementation," Korean Journal of Radiology, vol. 17, no. 5, p. 695, 2016. DOI: 10.3348/kjr.2016.17.5 695

[61] E. Aribal, D. Tureli, F. Kucukkaya, and H. Kaya, "Volume Navigation Technique for Ultrasound-Guided Biopsy of Breast Lesions Detected Only at MRI," American Journal of Roentgenology, vol. 208, no. 6, pp. 1400-1409, Jun. 2017. DOI: 10.2214/AJR.16.16808

[62] K. Nakashima, T. Uematsu, T. L. Harada, K. Takahashi, S. Nishimura, Y. Tadokoro, T. Hayashi, J. Watanabe, and T. Sugino, "MRI-detected breast lesions: clinical implications and evaluation based on MRI/ultrasonography fusion technology," Japanese Journal of Radiology, vol. 37, no. 10, pp. 685-693, Oct. 2019. DOI: 10.1007/s11604-019-00866-8

[63] M. A. Mazzei, L. Di Giacomo, A. Fausto, F. Gentili, F. G. Mazzei, and L. Volterrani, "Efficacy of Second-Look Ultrasound with MR Coregistration for Evaluating Additional Enhancing Lesions of the Breast: Review of the Literature," BioMed Research International, vol. 2018, pp. 1-8, Oct. 2018. DOI: 10.1155/2018/3896946 
[64] A. Fausto, G. Rizzatto, A. Preziosa, L. Gaburro, M. J. Washburn, D. Rubello, and L. Volterrani, "A new method to combine contrast-enhanced magnetic resonance imaging during live ultrasound of the breast using volume navigation technique: A study for evaluating feasibility, accuracy and reproducibility in healthy volunteers," European Journal of Radiology, vol. 81, no. 3, e332-e337, Mar. 2012. DOI: 10.1016/j.ejrad.2011. 11.001 .

[65] A. V. Nikolaev, L. De Jong, G. Weijers, V. Groenhuis, R. M. Mann, F. J. Siepel, B. M. Maris, S. Stramigioli, H. H. Hansen, and C. L. De Korte, "Quantitative Evaluation of an Automated Cone-based Breast Ultrasound Scanner for MRI - 3D US Image Fusion," IEEE Transactions on Medical Imaging, vol. 40, no. 4, pp. 1-1, Apr. 2021. DOI: $10.1109 /$ TMI . 2021.3050525

[66] N. Nyayapathi and J. Xia, "Photoacoustic imaging of breast cancer: a mini review of system design and image features," Journal of Biomedical Optics, vol. 24, no. 12, p. 1, Nov. 2019. DOI: $10.1117 / 1 . J B 0.24 .12 .121911$

[67] C. Hennersperger, B. Fuerst, S. Virga, O. Zettinig, B. Frisch, T. Neff, and N. Navab, "Towards MRI-Based Autonomous Robotic US Acquisitions: A First Feasibility Study," IEEE Transactions on Medical Imaging, vol. 36, no. 2, pp. 538-548, 2017. DOI: 10. 1109/TMI.2016.2620723 arXiv: 1607.08371

[68] T. E. Chemaly, F. J. Siepel, S. Rihana, V. Groenhuis, F. van der Heijden, and S. Stramigioli, "MRI and stereo vision surface reconstruction and fusion," in 2017 Fourth International Conference on Advances in Biomedical Engineering (ICABME), vol. 2017Octob, IEEE, Oct. 2017, pp. 1-4, ISBN: 978-1-5386-1642-0. DOI: 10.1109/ICABME. 2017 8167571

[69] P. Chatelain, A. Krupa, and N. Navab, "Confidence-Driven Control of an Ultrasound Probe," IEEE Transactions on Robotics, vol. 33, no. 6, pp. 1410-1424, Dec. 2017. DOI: 10.1109/TRO.2017.2723618

[70] Z. Jiang, M. Grimm, M. Zhou, J. Esteban, W. Simson, G. Zahnd, and N. Navab, "Automatic Normal Positioning of Robotic Ultrasound Probe Based Only on Confidence Map Optimization and Force Measurement," IEEE Robotics and Automation Letters, vol. 5, no. 2, pp. 1342-1349, Apr. 2020. DOI: 10.1109/LRA.2020.2967682

[71] Z. Jiang, M. Grimm, M. Zhou, Y. Hu, J. Esteban, and N. Navab, "Automatic ForceBased Probe Positioning for Precise Robotic Ultrasound Acquisition," IEEE Transactions on Industrial Electronics, vol. 0046, no. c, pp. 1-1, Nov. 2020. DOI: 10.1109/TIE 2020.3036215.

[72] R. Monfaredi, E. Wilson, B. Azizi koutenaei, B. Labrecque, K. Leroy, J. Goldie, E. Louis, D. Swerdlow, and K. Cleary, "Robot-assisted ultrasound imaging: Overview and development of a parallel telerobotic system," Minimally Invasive Therapy 6 Allied Technologies, vol. 24, no. 1, pp. 54-62, Jan. 2015. DOI: 10.3109/13645706.2014.992908

[73] S. Virga, O. Zettinig, M. Esposito, K. Pfister, B. Frisch, T. Neff, N. Navab, and C. Hennersperger, "Automatic force-compliant robotic ultrasound screening of abdominal aortic aneurysms," in 2016 IEEE/RSJ International Conference on Intelligent Robots and Systems (IROS), IEEE, Oct. 2016, pp. 508-513, ISBN: 978-1-5090-3762-9. DOI: 10.1109/IROS.2016.7759101

[74] M. Abayazid, P. Moreira, N. Shahriari, S. Patil, R. Alterovitz, and S. Misra, "Ultrasoundguided three-dimensional needle steering in biological tissue with curved surfaces," Medical Engineering 8 Physics, vol. 37, no. 1, pp. 145-150, Jan. 2015. DOI: 10.1016/j. medengphy.2014.10.005

[75] C. Nadeau and A. Krupa, "Intensity-Based Ultrasound Visual Servoing: Modeling and Validation With 2-D and 3-D Probes," IEEE Transactions on Robotics, vol. 29, no. 4, pp. 1003-1015, Aug. 2013. DOI: 10.1109/TRO.2013.2256690 
[76] Y. J. Kim, J. H. Seo, H. R. Kim, and K. G. Kim, "Development of a control algorithm for the ultrasound scanning robot (NCCUSR) using ultrasound image and force feedback," The International Journal of Medical Robotics and Computer Assisted Surgery, vol. 13, no. 2, e1756, Jun. 2017. DOI: 10.1002/rcs.1756

[77] P. Abolmaesumi, S. Salcudean, Wen-Hong Zhu, M. Sirouspour, and S. DiMaio, "Imageguided control of a robot for medical ultrasound," IEEE Transactions on Robotics and Automation, vol. 18, no. 1, pp. 11-23, Aug. 2002. DOI: 10.1109/70.988970

[78] R. Mebarki, A. Krupa, and F. Chaumette, "2-D Ultrasound Probe Complete Guidance by Visual Servoing Using Image Moments," IEEE Transactions on Robotics, vol. 26, no. 2, pp. 296-306, Apr. 2010. DOI: 10.1109/TRO.2010.2042533

[79] A. Krupa, G. Fichtinger, and G. D. Hager, "Real-time Motion Stabilization with B-mode Ultrasound Using Image Speckle Information and Visual Servoing," The International Journal of Robotics Research, vol. 28, no. 10, pp. 1334-1354, Oct. 2009. DOI: $10.1177 / 0278364909104066$

[80] R. Kojcev, A. Khakzar, B. Fuerst, O. Zettinig, C. Fahkry, R. DeJong, J. Richmon, R. Taylor, E. Sinibaldi, and N. Navab, "On the reproducibility of expert-operated and robotic ultrasound acquisitions," International Journal of Computer Assisted Radiology and Surgery, vol. 12, no. 6, pp. 1003-1011, 2017. DOI: 10.1007/s11548-017-1561-1.

[81] L. Santiago, B. E. Adrada, M. L. Huang, W. Wei, and R. P. Candelaria, "Breast cancer neoplastic seeding in the setting of image-guided needle biopsies of the breast," Breast Cancer Research and Treatment, vol. 166, no. 1, pp. 29-39, Nov. 2017. DOI: 10.1007/s10549-017-4401-7

[82] R. Tyagi and P. Dey, "Needle tract seeding: An avoidable complication," Diagnostic Cytopathology, vol. 42, no. 7, L. Pantanowitz, Ed., pp. 636-640, Jul. 2014. DOI: 10 1002/dc.23137

[83] N. Tanaiutchawoot, C. Wiratkapan, B. Treepong, and J. Suthakorn, "On the design of a biopsy needle-holding robot for a novel breast biopsy robotic navigation system," in The 4 th Annual IEEE International Conference on Cyber Technology in Automation, Control and Intelligent, IEEE, Jun. 2014, pp. 480-484, ISBN: 978-1-4799-3669-4. DOI: 10.1109/CYBER.2014.6917511

[84] N. Tanaiutchawoot, B. Treepong, C. Wiratkapan, and J. Suthakorn, "A path generation algorithm for biopsy needle insertion in a robotic breast biopsy navigation system," in 2014 IEEE International Conference on Robotics and Biomimetics (ROBIO 2014), IEEE, Dec. 2014, pp. 398-403, ISBN: 978-1-4799-7397-2. DOI: 10.1109/ROBIO. 2014 7090363

[85] M. C. Mahoney and M. S. Newell, "Breast Intervention: How I Do It," Radiology, vol. 268, no. 1, pp. 12-24, Jul. 2013. DOI: 10.1148/radiol.13120985

[86] H. H. G. Hansen, L. de Jong, R. Mann, F. Siepel, A. Nikolaev, E. Tagliabue, B. Maris, V. Groenhuis, M. Caballo, I. Sechopoulos, and C. L. de Korte, "Ultrasound-guided breast biopsy of ultrasound occult lesions using multimodality image co-registration and tissue displacement tracking," in Medical Imaging 2019: Ultrasonic Imaging and Tomography, N. V. Ruiter and B. C. Byram, Eds., SPIE, Mar. 2019, p. 45, ISBN: 9781510625570. DOI: $10.1117 / 12.2513630$

[87] N. Bluvol, A. Shaikh, A. Kornecki, D. Del Rey Fernandez, D. Downey, and A. Fenster, "A needle guidance system for biopsy and therapy using two-dimensional ultrasound," Medical Physics, vol. 35, no. 2, pp. 617-628, Jan. 2008. DoI: 10.1118/1.2829871 
[88] L. J. Brattain, C. Floryan, O. P. Hauser, M. Nguyen, R. J. Yong, S. B. Kesner, S. B. Corn, and C. J. Walsh, "Simple and effective ultrasound needle guidance system," in 2011 Annual International Conference of the IEEE Engineering in Medicine and Biology Society, IEEE, Aug. 2011, pp. 8090-8093, ISBN: 978-1-4577-1589-1. DOI: 10.1109/IEMBS.2011.6091995.

[89] J. Suthakorn, N. Tanaiutchawoot, C. Wiratkapan, and S. Ongwattanakul, "Breast biopsy navigation system with an assisted needle holder tool and 2D graphical user interface," European Journal of Radiology Open, vol. 5, no. July, pp. 93-101, Jul. 2018. DOI: $10.1016 /$ j.ejro.2018.07.001

[90] M. D'Souza, J. Gendreau, A. Feng, L. H. Kim, A. L. Ho, and A. Veeravagu, "RoboticAssisted Spine Surgery: History, Efficacy, Cost, And Future Trends," Robotic Surgery: Research and Reviews, vol. Volume 6, pp. 9-23, Nov. 2019. DOI: 10.2147/RSRR.S190720

[91] S. Amack, M. F. Rox, M. Emerson, R. J. Webster, R. Alterovitz, A. Kuntz, J. Mitchell, T. E. Ertop, J. Gafford, F. Maldonado, and J. Akulian, "Design and control of a compact modular robot for transbronchial lung biopsy," in Medical Imaging 2019: Image-Guided Procedures, Robotic Interventions, and Modeling, B. Fei and C. A. Linte, Eds., SPIE, Mar. 2019, p. 17, ISBN: 9781510625495. DOI: 10.1117/12.2513967

[92] G. Minchev, G. Kronreif, M. Martínez-Moreno, C. Dorfer, A. Micko, A. Mert, B. Kiesel, G. Widhalm, E. Knosp, and S. Wolfsberger, "A novel miniature robotic guidance device for stereotactic neurosurgical interventions: preliminary experience with the iSYS1 robot," Journal of Neurosurgery, vol. 126, no. 3, pp. 985-996, Mar. 2017. DOI: 10.3171/2016.1. JNS152005

[93] D. Stoianovici, C. Kim, D. Petrisor, C. Jun, S. Lim, M. W. Ball, A. Ross, K. J. Macura, and M. E. Allaf, "MR Safe Robot, FDA Clearance, Safety and Feasibility of Prostate Biopsy Clinical Trial," IEEE/ASME Transactions on Mechatronics, vol. 22, no. 1, pp. 115-126, Feb. 2017. DOI: 10.1109/TMECH.2016.2618362

[94] E. Franco, D. Brujic, M. Rea, W. M. Gedroyc, and M. Ristic, "Needle-Guiding Robot for Laser Ablation of Liver Tumors Under MRI Guidance," IEEE/ASME Transactions on Mechatronics, vol. 21, no. 2, pp. 931-944, Apr. 2016. DOI: 10.1109/TMECH. 2015.2476556

[95] M. Z. Mahmoud, M. Aslam, M. Alsaadi, M. A. Fagiri, and B. Alonazi, "Evolution of Robot-assisted ultrasound-guided breast biopsy systems," Journal of Radiation Research and Applied Sciences, vol. 11, no. 1, pp. 89-97, Jan. 2018. DoI: 10.1016/j. jrras.2017.11.005

[96] Z. Xinran, D. Haiyan, L. Mingyue, and Z. Yongde, "Breast intervention surgery robot under image navigation: A review," Advances in Mechanical Engineering, vol. 13, no. 6, p. 168781402110281 , Jun. 2021. DOI: 10.1177/16878140211028113

[97] G. Megali, O. Tonet, C. Stefanini, M. Boccadoro, V. Papaspyropoulos, L. Angelini, and P. Dario, "A Computer-Assisted Robotic Ultrasound-Guided Biopsy System for VideoAssisted Surgery," in Niessen W.J., Viergever M.A. (eds) Medical Image Computing and Computer-Assisted Intervention - MICCAI 2001. MICCAI 2001. Lecture Notes in Computer Science, vol. 2208, Springer, 2001, pp. 343-350, ISBN: 3540426973. DOI: 10.1007/3-540-45468-3_41

[98] J. Kettenbach, G. Kronreif, M. Figl, M. Fürst, W. Birkfellner, R. Hanel, and H. Bergmann, "Robot-assisted biopsy using ultrasound guidance: initial results from in vitro tests," European Radiology, vol. 15, no. 4, pp. 765-771, Apr. 2005. DOI: 10.1007/s00330-004-2487-x

[99] R. Kojcev, B. Fuerst, O. Zettinig, J. Fotouhi, S. C. Lee, B. Frisch, R. Taylor, E. Sinibaldi, and N. Navab, "Dual-robot ultrasound-guided needle placement: closing the planning-imaging-action loop," International Journal of Computer Assisted Radiology and Surgery, vol. 11, no. 6, pp. 1173-1181, Jun. 2016. DoI: 10.1007/s11548-016-1408-1 
[100] J. Hong, T. Dohi, M. Hashizume, K. Konishi, and N. Hata, "An ultrasound-driven needle-insertion robot for percutaneous cholecystostomy," Physics in Medicine and Biology, vol. 49, no. 3, pp. 441-455, Feb. 2004. DOI: 10.1088/0031-9155/49/3/007

[101] G. J. Vrooijink, M. Abayazid, and S. Misra, "Real-time three-dimensional flexible needle tracking using two-dimensional ultrasound," in 2013 IEEE International Conference on Robotics and Automation, IEEE, May 2013, pp. 1688-1693, ISBN: 978-1-4673-5643-5. DOI: $10.1109 /$ ICRA.2013.6630797

[102] T. R. Nelson, A. Tran, H. Fakourfar, and J. Nebeker, "Positional Calibration of an Ultrasound Image-Guided Robotic Breast Biopsy System," Journal of Ultrasound in Medicine, vol. 31, no. 3, pp. 351-359, Mar. 2012. DoI: 10.7863/jum.2012.31.3.351

[103] M. Hatano, Y. Kobayashi, R. Hamano, M. Suzuki, Y. Shiraishi, T. Yambe, K. Konishi, M. Hashizume, and M. G. Fujie, "In vitro and in vivo validation of robotic palpationbased needle insertion method for breast tumor treatment," in 2011 IEEE International Conference on Robotics and Automation, IEEE, May 2011, pp. 392-397, ISBN: 978-161284-386-5. DOI: 10.1109/ICRA.2011.5979896

[104] N. Abolhassani, R. Patel, and M. Moallem, "Needle insertion into soft tissue: A survey," Medical Engineering \& Physics, vol. 29, no. 4, pp. 413-431, May 2007. DOI: 10.1016/j.medengphy.2006.07.003

[105] S. G. Orel and M. D. Schnall, "MR Imaging of the Breast for the Detection, Diagnosis, and Staging of Breast Cancer," Radiology, vol. 220, no. 1, pp. 13-30, Jul. 2001. DOI: 10.1148/radiology.220.1.r01j13113

[106] C. T. Coffin, "Work-related musculoskeletal disorders in sonographers: A review of causes and types of injury and best practices for reducing injury risk," Reports in Medical Imaging, vol. 7, no. 1, pp. 15-26, 2014. DOI: 10.2147/RMI.S34724

[107] E. Tagliabue, D. Dall'Alba, E. Magnabosco, C. Tenga, I. Peterlik, and P. Fiorini, "Position-based modeling of lesion displacement in ultrasound-guided breast biopsy," International Journal of Computer Assisted Radiology and Surgery, vol. 14, no. 8, pp. 1329-1339, Aug. 2019. DOI: 10.1007/s11548-019-01997-z.

[108] V. Kumar, J. M. Webb, A. Gregory, M. Denis, D. D. Meixner, M. Bayat, D. H. Whaley, M. Fatemi, and A. Alizad, "Automated and real-time segmentation of suspicious breast masses using convolutional neural network," PLoS ONE, vol. 13, no. 5, pp. 1-18, 2018. DOI: $10.1371 /$ journal.pone.0195816

[109] H. Karimi, A. Fenster, and A. Samani, "A real-time method for breast cancer diagnosis using optical flow," in Medical Imaging 2009: Biomedical Applications in Molecular, Structural, and Functional Imaging, X. P. Hu and A. V. Clough, Eds., vol. 7262, Feb. 2009, 72621A, ISBN: 9780819475138 . DOI: $10.1117 / 12.813896$

[110] M. Kaya, E. Senel, A. Ahmad, and O. Bebek, "Visual tracking of multiple moving targets in 2D ultrasound guided robotic percutaneous interventions," in 2017 IEEE International Conference on Robotics and Automation (ICRA), IEEE, May 2017, pp. 1996-2002, ISBN: 978-1-5090-4633-1. DOI: 10.1109/ICRA.2017.7989231

[111] V. Mallapragada, N. Sarkar, and T. K. Podder, "A robotic system for real-time tumor manipulation during image guided breast biopsy," Proceedings of the 7th IEEE International Conference on Bioinformatics and Bioengineering, BIBE, pp. 204-210, 2007. DOI: $10.1109 /$ BIBE. 2007.4375566

[112] V. G. Mallapragada, N. Sarkar, and T. K. Podder, "Robotic system for tumor manipulation and ultrasound image guidance during breast biopsy," in 2008 30th Annual International Conference of the IEEE Engineering in Medicine and Biology Society, IEEE, Aug. 2008, pp. 5589-5592, ISBN: 978-1-4244-1814-5. DOI: 10.1109/IEMBS. 2008.4650481 
[113] - "Autonomous coordination of imaging and tumor manipulation for robot assisted breast biopsy," Proceedings of the 2nd Biennial IEEE/RAS-EMBS International Conference on Biomedical Robotics and Biomechatronics, BioRob 2008, pp. 676-681, 2008. DOI: 10.1109/BIOROB. 2008.4762815

[114] V. Mallapragada, N. Sarkar, and T. Podder, "Robot-Assisted Real-Time Tumor Manipulation for Breast Biopsy," IEEE Transactions on Robotics, vol. 25, no. 2, pp. 316-324, Apr. 2009. DOI: 10.1109/TRO.2008.2011418.

[115] H. Su, D. C. Cardona, W. Shang, A. Camilo, G. A. Cole, D. C. Rucker, R. J. Webster, and G. S. Fischer, "A MRI-guided concentric tube continuum robot with piezoelectric actuation: A feasibility study," Proceedings - IEEE International Conference on Robotics and Automation, pp. 1939-1945, 2012. DOI: 10.1109/ICRA.2012.6224550.

[116] V. Groenhuis, F. J. Siepel, J. Veltman, J. K. van Zandwijk, and S. Stramigioli, "Stormram 4: An MR Safe Robotic System for Breast Biopsy," Annals of Biomedical Engineering, vol. 46, no. 10, pp. 1686-1696, Oct. 2018. DOI: 10.1007/s10439-018-2051-5

[117] Z. Dong, Z. Guo, K. H. Lee, G. Fang, W. L. Tang, H. C. Chang, D. T. M. Chan, and K. W. Kwok, "High-Performance Continuous Hydraulic Motor for MR Safe Robotic Teleoperation," IEEE Robotics and Automation Letters, vol. 4, no. 2, pp. 1964-1971, 2019. DOI: 10.1109/LRA.2019.2899189

[118] S. T. Kim, Y. Kim, and J. Kim, "Design of an MR-compatible biopsy needle manipulator using pull-pull cable transmission," International Journal of Precision Engineering and Manufacturing, vol. 17, no. 9, pp. 1129-1137, 2016. DOI: 10.1007/s12541-016-0137-2

[119] L. de Jong, M. K. Welleweerd, J. C. van Zelst, F. J. Siepel, S. Stramigioli, R. M. Mann, C. L. de Korte, and J. J. Fütterer, "Production and clinical evaluation of breast lesion skin markers for automated three-dimensional ultrasonography of the breast: a pilot study," European Radiology, vol. 30, no. 6, pp. 3356-3362, Jun. 2020. DOI: 10.1007/s00330-020-06695-y.

[120] A. V. Nikolaev, L. de Jong, V. Groenhuis, M. K. Welleweerd, F. J. Siepel, S. Stramigioli, H. H. G. Hansen, and C. L. de Korte, "Quantitative Evaluation of Automated RobotAssisted Volumetric Breast Ultrasound," in 2020 IEEE International Ultrasonics Symposium (IUS), IEEE, Sep. 2020, pp. 1-4, ISBN: 978-1-7281-5448-0. DOI: 10.1109/ IUS46767.2020.9251310

[121] V. Groenhuis, E. Tagliabue, M. K. Welleweerd, F. J. Siepel, J. D. Munoz Osorio, B. M. Maris, D. Dall'Alba, U. Zimmermann, P. Fiorini, and S. Stramigioli, "Deformation Compensation in Robotically-Assisted Breast Biopsy," in 11th International Conference on Information Processing in Computer-Assisted Interventions, Jun. 2020.

[122] V. Groenhuis, A. Nikolaev, S. H. G. Nies, M. K. Welleweerd, L. de Jong, H. H. G. Hansen, F. J. Siepel, C. L. de Korte, and S. Stramigioli, "3-D Ultrasound Elastography Reconstruction Using Acoustically Transparent Pressure Sensor on Robotic Arm," IEEE Transactions on Medical Robotics and Bionics, vol. 3, no. 1, pp. 265-268, Feb. 2021. DOI: 10.1109/TMRB.2020.3042982

[123] V. Groenhuis, F. J. Siepel, M. K. Welleweerd, J. Veltman, and S. Stramigioli, "Sunram 5: An MR Safe Robotic System for Breast Biopsy," in The Hamlyn Symposium, 2018, pp. 85-86. DOI: $10.31256 / \mathrm{hsmr} 2018.43$

[124] M. Rahimzadeh, A. R. Baghestani, M. R. Gohari, and M. A. Pourhoseingholi, "Estimation of the Cure Rate in Iranian Breast Cancer Patients," Asian Pacific Journal of Cancer Prevention, vol. 15, no. 12, pp. 4839-4842, Jun. 2014. DOI: 10.7314/APJCP 2014.15.12.4839 
[125] F. Pediconi, C. Catalano, A. Roselli, V. Dominelli, S. Cagioli, A. Karatasiou, A. Pronio, M. A. Kirchin, and R. Passariello, "The Challenge of Imaging Dense Breast Parenchyma," Investigative Radiology, vol. 44, no. 7, pp. 412-421, Jul. 2009. DOI: 10.1097/RLI.0b013e3181a53654

[126] C. M. Sommerich, S. A. Lavender, K. Evans, E. Sanders, S. Joines, S. Lamar, R. Z. Radin Umar, W.-t. Yen, J. Li, S. Nagavarapu, and J. A. Dickerson, "Collaborating with cardiac sonographers to develop work-related musculoskeletal disorder interventions," Ergonomics, vol. 59, no. 9, pp. 1193-1204, Sep. 2016. DOI: 10.1080/00140139.2015 1116613

[127] K. Liang, A. J. Rogers, E. D. Light, D. von Allmen, and S. W. Smith, "Simulation of Autonomous Robotic Multiple-Core Biopsy by 3D Ultrasound Guidance," Ultrasonic Imaging, vol. 32, no. 2, pp. 118-127, Apr. 2010. DOI: 10.1177/016173461003200205

[128] - "Three-Dimensional Ultrasound Guidance of Autonomous Robotic Breast Biopsy: Feasibility Study," Ultrasound in Medicine \& Biology, vol. 36, no. 1, pp. 173-177, Jan. 2010. DOI: $10.1016 /$ j.ultrasmedbio.2009.08.014

[129] R. Spoor, M. Abayazid, F. Siepel, V. Groenhuis, and S. Stramigioli, "Design and evaluation of a robotic needle steering manipulator for image-guided biopsy," in $B M E$ 2017, vol. 6, 2017.

[130] Y. Kobayashi, A. Onishi, H. Watanabe, T. Hoshi, K. Kawamura, M. Hashizume, and M. G. Fujie, "Development of an integrated needle insertion system with image guidance and deformation simulation," Computerized Medical Imaging and Graphics, vol. 34, no. 1, pp. 9-18, Jan. 2010. DOI: 10.1016/j.compmedimag.2009.08.008

[131] M. Kaya, E. Senel, A. Ahmad, O. Orhan, and O. Bebek, "Real-time needle tip localization in 2D ultrasound images for robotic biopsies," in 2015 International Conference on Advanced Robotics (ICAR), IEEE, Jul. 2015, pp. 47-52, ISBN: 978-1-4673-7509-2. DOI: $10.1109 /$ ICAR. 2015.7251432

[132] A. O. Andrade, A. A. Pereira, S. Walter, R. Almeida, R. Loureiro, D. Compagna, and P. J. Kyberd, "Bridging the gap between robotic technology and health care," Biomedical Signal Processing and Control, vol. 10, no. 1, pp. 65-78, Mar. 2014. DOI: 10.1016/j.bspc.2013.12.009

[133] R. M. Murray, Z. Li, and S. S. Sastry, A Mathematical Introduction to Robotic Manipulation. CRC Press, Dec. 2017, ISBN: 9781315136370. DOI: 10.1201/9781315136370

[134] S.-Y. Huang, J. M. Boone, K. Yang, N. J. Packard, S. E. McKenney, N. D. Prionas, K. K. Lindfors, and M. J. Yaffe, "The characterization of breast anatomical metrics using dedicated breast CT," Medical Physics, vol. 38, no. 4, pp. 2180-2191, Mar. 2011. DOI: $10.1118 / 1.3567147$.

[135] Y. Xu, Q. Zhang, and G. Liu, "Cutting performance orthogonal test of single plane puncture biopsy needle based on puncture force," in AIP Conference Proceedings, 2017, p. 030016 , ISBN: 9780735415041 . DOI: $10.1063 / 1.4981581$

[136] M. Abayazid, J. op den Buijs, C. L. de Korte, and S. Misra, "Effect of skin thickness on target motion during needle insertion into soft-tissue phantoms," in 20124 th IEEE RAS \& EMBS International Conference on Biomedical Robotics and Biomechatronics (BioRob), IEEE, Jun. 2012, pp. 755-760, ISBN: 978-1-4577-1200-5. DOI: 10.1109/BioRob 2012.6290841

[137] M. Katna. "Thick walled cylinders." (2019), [Online]. Available: http://www . engr mun . ca/\%7B \%7Dkatna/5931/Thick\%20Walled\%20Cylinders (corrected).pdf (visited on 04/24/2019).

[138] J. Barber, "Thick-walled Cylinders and Disks," in Solid Mechanics and Its Applications, G. M. Gladwell, Ed., 2nd, vol. 175, Springer, Dordrecht, 2011, pp. 449-486, ISBN: 9789400702943. DOI: $10.1007 / 978-94-007-0295-0 \_10$ 
[139] M. K. Welleweerd, A. G. de Groot, S. O. H. de Looijer, F. J. Siepel, and S. Stramigioli, "Automated robotic breast ultrasound acquisition using ultrasound feedback," in 2020 IEEE International Conference on Robotics and Automation (ICRA), IEEE, May 2020, pp. 9946-9952, ISBN: 978-1-7281-7395-5. DOI: 10.1109/ICRA40945.2020.9196736

[140] K. Jovanovic, A. Schwier, E. Matheson, M. Xiloyannis, E. Rozeboom, N. Hochhausen, B. Vermeulen, B. Graf, P. Wolf, Z. Nawrat, J. Escuder, M. Mechelinck, B. Sorensen, P. R. Boscolo, M. Obach, S. Tognarelli, M. Jankovic, C. Leroux, G. Ferrigno, F. J. Siepel, and S. Stramigioli, "Digital Innovation Hubs in Health-Care Robotics Fighting COVID-19: Novel Support for Patients and Health-Care Workers Across Europe," IEEE Robotics \& Automation Magazine, vol. 28, no. 1, pp. 40-47, Mar. 2021. DOI: 10.1109/MRA.2020.3044965

[141] J. D. Muñoz Osorio, F. Castañeda, F. Allmendinger, and U. E. Zimmermann, "Time Invariant Motion Controller for Physical Human Robot Interaction," in Volume 5A: 43rd Mechanisms and Robotics Conference, vol. 5A-2019, American Society of Mechanical Engineers, Aug. 2019, ISBN: 978-0-7918-5923-0. DOI: 10.1115/DETC2019-98031

[142] Y. Nakamura, Advanced Robotics: Redundancy and Optimization. Addison-Wesley, 1991, ISBN: 0-201-15198-7.

[143] O. Khatib, "Real-time obstacle avoidance for manipulators and mobile robots," in Proceedings. 1985 IEEE International Conference on Robotics and Automation, vol. 2, Institute of Electrical and Electronics Engineers, 1985, pp. 500-505. DoI: 10.1109/ ROBOT.1985.1087247

[144] H. Han and J. Park, "Robot Control near Singularity and Joint Limit Using a Continuous Task Transition Algorithm," International Journal of Advanced Robotic Systems, vol. 10, no. 10, p. 346, Oct. 2013. DOI: 10.5772/56714

[145] S. Hjorth, J. Lachner, S. Stramigioli, O. Madsen, and D. Chrysostomou, "An Energybased Approach for the Integration of Collaborative Redundant Robots in Restricted Work Environments," in 2020 IEEE/RSJ International Conference on Intelligent Robots and Systems (IROS), IEEE, Oct. 2020, pp. 7152-7158, ISBN: 978-1-7281-6212-6. DOI: $10.1109 /$ IROS45743.2020.9341561

[146] J. D. Muñoz Osorio, M. D. Fiore, and F. Allmendinger, "Operational Space Formulation Under Joint Constraints," in ASME 2018 International Design Engineering Technical Conferences and Computers and Information in Engineering Conference, American Society of Mechanical Engineers, Aug. 2018, pp. 1-10, ISBN: 978-0-7918-5181-4. DOI: 10.1115/DETC2018-86058.

[147] F. Flacco, A. De Luca, and O. Khatib, "Control of Redundant Robots Under Hard Joint Constraints: Saturation in the Null Space," IEEE Transactions on Robotics, vol. 31, no. 3, pp. 637-654, Jun. 2015. DOI: 10.1109/TRO.2015.2418582

[148] M. Rauscher, M. Kimmel, and S. Hirche, "Constrained robot control using control barrier functions," in 2016 IEEE/RSJ International Conference on Intelligent Robots and Systems (IROS), vol. 2016-Novem, IEEE, Oct. 2016, pp. 279-285, ISBN: 978-1-50903762-9. DOI: 10.1109/IROS.2016.7759067.

[149] D. Papageorgiou, A. Atawnih, and Z. Doulgeri, "A passivity based control signal guaranteeing joint limit avoidance in redundant robots," in 2016 24th Mediterranean Conference on Control and Automation (MED), IEEE, Jun. 2016, pp. 569-574, ISBN: 978-1-4673-8345-5. DOI: 10.1109/MED.2016.7535862

[150] C. Ott, Cartesian Impedance Control of Redundant and Flexible-Joint Robots, ser. Springer Tracts in Advanced Robotics. Berlin, Heidelberg: Springer Berlin Heidelberg, 2008, vol. 49, ISBN: 978-3-540-69253-9. DOI: 10.1007/978-3-540-69255-3 
[151] H. Sadeghian, M. Keshmiri, L. Villani, and B. Siciliano, "Null-space impedance control with disturbance observer," IEEE International Conference on Intelligent Robots and Systems, pp. 2795-2800, 2012. DOI: 10.1109/IROS.2012.6385690.

[152] H. Sadeghian, L. Villani, M. Keshmiri, and B. Siciliano, "Task-space control of robot manipulators with null-space compliance," IEEE Transactions on Robotics, 2014. DOI: 10.1109/TRO.2013.2291630

[153] C. Ott, A. Kugi, and Y. Nakamura, "Resolving the problem of non-integrability of nullspace velocities for compliance control of redundant manipulators by using semidefinite Lyapunov functions," Proceedings - IEEE International Conference on Robotics and Automation, no. June, pp. 1999-2004, 2008. DOI: 10.1109/ROBOT.2008.4543500

[154] F. Vigoriti, F. Ruggiero, V. Lippiello, and L. Villani, "Control of redundant robot arms with null-space compliance and singularity-free orientation representation," Robotics and Autonomous Systems, vol. 100, pp. 186-193, Feb. 2018. DOI: 10.1016/j.robot 2017.11 .007

[155] S. Stramigioli, Modeling and IPC control of interactive mechanical systems - A coordinate-free approach, ser. Lecture Notes in Control and Information Sciences. London: Springer-Verlag London, 2001, vol. 266, ISBN: 978-1-85233-395-9. DOI: 10.1007/ BFb0110400.

[156] A. Albu-Schaffer, C. Ott, U. Frese, and G. Hirzinger, "Cartesian impedance control of redundant robots: recent results with the DLR-light-weight-arms," in 2003 IEEE International Conference on Robotics and Automation (Cat. No.03CH37422), vol. 3, IEEE, 2003, pp. 3704-3709, ISBN: 0-7803-7736-2. DOI: 10.1109/ROBOT.2003.1242165

[157] J. Lachner, V. Schettino, F. Allmendinger, M. D. Fiore, F. Ficuciello, B. Siciliano, and S. Stramigioli, "The influence of coordinates in robotic manipulability analysis," Mechanism and Machine Theory, vol. 146, no. 688188, p. 103722 , Apr. 2020. DOI: 10.1016/j.mechmachtheory.2019.103722

[158] D. Dresscher, Y. Brodskiy, P. C. Breedveld, J. F. Broenink, and S. Stramigioli, "Modeling of the youBot in a serial link structure using twists and wrenches in a bond graph," in International conference on simulation, modeling and programming for autonomous robots, 2010, pp. 385-400, ISBN: 978-3-00-032863-3.

[159] G. Schreiber, A. Stemmer, and R. Bischoff, "The fast research interface for the kuka lightweight robot," in IEEE Workshop on Innovative Robot Control Architectures for Demanding (Research) Applications How to Modify and Enhance Commercial Controllers (ICRA 2010), 2010, pp. 15-21.

[160] G. Raiola, C. A. Cardenas, T. S. Tadele, T. de Vries, and S. Stramigioli, "Development of a Safety- and Energy-Aware Impedance Controller for Collaborative Robots," IEEE Robotics and Automation Letters, vol. 3, no. 2, pp. 1237-1244, Apr. 2018. DOI: 10 1109/LRA. 2018.2795639.

[161] F. D. Nandan and B. A. Alladin, "The Role of Ultrasound as a Diagnostic Tool for Breast Cancer in the Screening of Younger Women (Age 25-38) in Guyana," Journal of Medical Diagnostic Methods, vol. 07, no. 03, Jan. 2018. DOI: 10.4172/2168-9784.1000273

[162] Q. Huang and Z. Zeng, "A Review on Real-Time 3D Ultrasound Imaging Technology," BioMed Research International, vol. 2017, pp. 1-20, Mar. 2017. DoI: 10.1155/2017/ 6027029 .

[163] Q. Huang, B. Wu, J. Lan, and X. Li, "Fully Automatic Three-Dimensional Ultrasound Imaging Based on Conventional B-Scan," IEEE Transactions on Biomedical Circuits and Systems, vol. 12, no. 2, pp. 426-436, Apr. 2018. DOI: 10.1109/TBCAS.2017.2782815 
[164] H. T. Sen, M. A. L. Bell, I. Iordachita, J. Wong, and P. Kazanzides, "A cooperatively controlled robot for ultrasound monitoring of radiation therapy," in 2013 IEEE/RSJ International Conference on Intelligent Robots and Systems, IEEE, Nov. 2013, pp. 30713076, ISBN: 978-1-4673-6358-7. DOI: 10.1109/IROS.2013.6696791

[165] N. Hungr, M. Baumann, J.-A. Long, and J. Troccaz, "A 3-D Ultrasound Robotic Prostate Brachytherapy System With Prostate Motion Tracking," IEEE Transactions on Robotics, vol. 28, no. 6, pp. 1382-1397, Dec. 2012. DOI: 10.1109/TR0.2012.2203051

[166] S. Virga, R. Göbl, M. Baust, N. Navab, and C. Hennersperger, "Use the force: deformation correction in robotic 3D ultrasound," International Journal of Computer Assisted Radiology and Surgery, vol. 13, no. 5, pp. 619-627, May 2018. DOI: $10.1007 /$ s11548018-1716-8

[167] P. Chatelain, A. Krupa, and N. Navab, "Optimization of ultrasound image quality via visual servoing," in 2015 IEEE International Conference on Robotics and Automation (ICRA), IEEE, May 2015, pp. 5997-6002, ISBN: 978-1-4799-6923-4. DOI: 10.1109/ICRA. 2015.7140040.

[168] - "Confidence-driven control of an ultrasound probe: Target-specific acoustic window optimization," in 2016 IEEE International Conference on Robotics and Automation (ICRA), vol. 2016-June, IEEE, May 2016, pp. 3441-3446, ISBN: 978-1-4673-8026-3. DOI: 10.1109/ICRA.2016.7487522

[169] R. Nakadate, J. Solis, A. Takanishi, E. Minagawa, M. Sugawara, and K. Niki, "Outof-plane visual servoing method for tracking the carotid artery with a robot-assisted ultrasound diagnostic system," in 2011 IEEE International Conference on Robotics and Automation, IEEE, May 2011, pp. 5267-5272, ISBN: 978-1-61284-386-5. DOI: 10 1109/ICRA.2011.5979594.

[170] M. K. Welleweerd, F. J. Siepel, V. Groenhuis, J. Veltman, and S. Stramigioli, "Design of an end-effector for robot-assisted ultrasound-guided breast biopsies," International Journal of Computer Assisted Radiology and Surgery, vol. 15, no. 4, pp. 681-690, Apr. 2020. DOI: $10.1007 / \mathrm{s} 11548-020-02122-1$

[171] T. Möller and B. Trumbore, "Fast, Minimum Storage Ray-Triangle Intersection," Journal of Graphics Tools, vol. 2, no. 1, pp. 21-28, Jan. 1997. DOI: 10.1080/10867651. 1997.10487468 arXiv: arXiv:1011.1669v3

[172] A. Karamalis, W. Wein, T. Klein, and N. Navab, "Ultrasound confidence maps using random walks," Medical Image Analysis, vol. 16, no. 6, pp. 1101-1112, Aug. 2012. DOI: 10.1016/j.media.2012.07.005

[173] S. Stramigioli, "Energy-aware robotics," in Mathematical Control Theory I, ser. Lecture Notes in Control and Information Sciences, M. K. Camlibel, A. A. Julius, R. Pasumarthy, and J. M. Scherpen, Eds., vol. 461, Cham: Springer International Publishing, 2015, pp. 37-49, ISBN: 978-3-319-20987-6. DOI: 10.1007/978-3-319-20988-3

[174] M. Nothacker, V. Duda, M. Hahn, M. Warm, F. Degenhardt, H. Madjar, S. Weinbrenner, and U.-S. Albert, "Early detection of breast cancer: benefits and risks of supplemental breast ultrasound in asymptomatic women with mammographically dense breast tissue. A systematic review," BMC Cancer, vol. 9, no. 1, p. 335, Dec. 2009. Dor: 10.1186/14712407-9-335

[175] S. Liu, Y. Wang, X. Yang, B. Lei, L. Liu, S. X. Li, D. Ni, and T. Wang, "Deep Learning in Medical Ultrasound Analysis: A Review," Engineering, vol. 5, no. 2, pp. 261-275, Apr. 2019. DOI: $10.1016 / \mathrm{j}$.eng.2018.11.020

[176] F. Mohamed and C. Vei Siang, "A Survey on 3D Ultrasound Reconstruction Techniques," in Artificial Intelligence - Applications in Medicine and Biology, IntechOpen, Jul. 2019, ch. 4. DOI: $10.5772 /$ intechopen. 81628 
[177] C. Graumann, B. Fuerst, C. Hennersperger, F. Bork, and N. Navab, "Robotic ultrasound trajectory planning for volume of interest coverage," in 2016 IEEE International Conference on Robotics and Automation (ICRA), vol. 2016-June, IEEE, May 2016, pp. 736-741, ISBN: 978-1-4673-8026-3. DOI: 10.1109/ICRA.2016.7487201

[178] K. Mathiassen, J. E. Fjellin, K. Glette, P. K. Hol, and O. J. Elle, "An Ultrasound Robotic System Using the Commercial Robot UR5," Frontiers in Robotics and AI, vol. 3, no. February, pp. 1-16, Feb. 2016. DOI: 10.3389/frobt.2016.00001

[179] A. S. B. Mustafa, T. Ishii, Y. Matsunaga, R. Nakadate, H. Ishii, K. Ogawa, A. Saito, M. Sugawara, K. Niki, and A. Takanishi, "Development of robotic system for autonomous liver screening using ultrasound scanning device," in 2013 IEEE International Conference on Robotics and Biomimetics (ROBIO), IEEE, Dec. 2013, pp. 804-809, ISBN: 978-1-4799-2744-9. DOI: 10.1109/ROBIO.2013.6739561

[180] O. Khatib, "A unified approach for motion and force control of robot manipulators: The operational space formulation," IEEE Journal on Robotics and Automation, vol. 3, no. 1, pp. 43-53, Feb. 1987. DOI: 10.1109/JRA.1987.1087068

[181] N. Chernov, $C++$ code for circle fitting algorithms.

[182] A. Gefen and B. Dilmoney, "Mechanics of the normal woman's breast," Technology and Health Care, vol. 15, no. 4, pp. 259-271, Jul. 2007. DOI: 10.3233/THC-2007-15404

[183] A. Samani, J. Zubovits, and D. Plewes, "Elastic moduli of normal and pathological human breast tissues: An inversion-technique-based investigation of 169 samples," Physics in Medicine and Biology, vol. 52, no. 6, pp. 1565-1576, 2007. DOI: 10.1088/00319155/52/6/002

[184] W. Li, B. Belmont, and A. Shih, "Design and Manufacture of Polyvinyl Chloride (PVC) Tissue Mimicking Material for Needle Insertion," Procedia Manufacturing, vol. 1, pp. 866-878, Jun. 2015. DOI: 10.1016/j.promfg.2015.09.078

[185] B. Eiben, V. Vavourakis, J. H. Hipwell, S. Kabus, C. Lorenz, T. Buelow, and D. J. Hawkes, "Breast deformation modelling: comparison of methods to obtain a patient specific unloaded configuration," in Medical Imaging 2014: Image-Guided Procedures, Robotic Interventions, and Modeling, Z. R. Yaniv and D. R. Holmes, Eds., vol. 9036, Mar. 2014, p. 903615, ISBN: 9780819498298. DOI: 10.1117/12.2043607

[186] Z. Neubach and M. Shoham, "Ultrasound-Guided Robot for Flexible Needle Steering," IEEE Transactions on Biomedical Engineering, vol. 57, no. 4, pp. 799-805, Apr. 2010. DOI: $10.1109 /$ TBME. 2009.2030169

[187] B. D. Lucas and T. Kanade, "Iterative Image Registration Technique With an Application To Stereo Vision," in 7th International Joint Conference on Artificial Intelligence (IJCAI' 81), vol. 2, Morgan Kaufmann Publishers Inc., 1981, pp. 674-679.

[188] J.-Y. Bouget, "Pyramidal implementation of the affine Lucas Kanade feature tracker," Intel Corporation, Microprocessor Research Labs, 2000.

[189] J. Canny, "A Computational Approach to Edge Detection," IEEE Transactions on Pattern Analysis and Machine Intelligence, vol. PAMI-8, no. 6, pp. 679-698, Nov. 1986. DOI: 10.1109/TPAMI.1986.4767851

[190] R. O. Duda and P. E. Hart, "Use of the Hough transformation to detect lines and curves in pictures," Communications of the ACM, vol. 15, no. 1, pp. 11-15, Jan. 1972. DOI: $10.1145 / 361237.361242$

[191] N. Hata, P. Moreira, and G. Fischer, "Robotics in MRI-Guided Interventions," Topics in Magnetic Resonance Imaging, vol. 27, no. 1, pp. 19-23, Feb. 2018. DOI: 10.1097/ RMR.0000000000000159. 
[192] F. G. Shellock, T. O. Woods, and J. V. Crues, "MR labeling information for implants and devices: Explanation of terminology," Radiology, vol. 253, no. 1, pp. 26-30, 2009. DOI: $10.1148 /$ radiol.2531091030

[193] H. Su, I. I. Iordachita, J. Tokuda, N. Hata, X. Liu, R. Seifabadi, S. Xu, B. Wood, and G. S. Fischer, "Fiber-Optic Force Sensors for MRI-Guided Interventions and Rehabilitation: A Review," IEEE Sensors Journal, vol. 17, no. 7, pp. 1952-1963, Apr. 2017. DOI: 10.1109/JSEN. 2017.2654489

[194] F. Taffoni, D. Formica, P. Saccomandi, G. Pino, and E. Schena, "Optical Fiber-Based MR-Compatible Sensors for Medical Applications: An Overview," Sensors, vol. 13, no. 10, pp. 14105-14120, Oct. 2013. DOI: 10.3390/s131014105.

[195] R. Gassert, D. Chapuis, H. Bleuler, and E. Burdet, "Sensors for Applications in Magnetic Resonance Environments," IEEE/ASME Transactions on Mechatronics, vol. 13, no. 3, pp. 335-344, Jun. 2008. DOI: 10.1109/TMECH.2008.924113

[196] H. Elhawary, A. Zivanovic, M. Rea, B. L. Davies, C. Besant, D. McRobbie, N. M. Desouza, I. Young, and M. U. Lampérth, "A modular approach to MRI-compatible robotics," IEEE Engineering in Medicine and Biology Magazine, vol. 27, no. 3, pp. 3541, 2008. DOI: 10.1109/EMB.2007.910260

[197] R. Gassert, R. Moser, E. Burdet, and H. Bleuler, "MRI/fMRI-compatible robotic system with force feedback for interaction with human motion," IEEE/ASME Transactions on Mechatronics, vol. 11, no. 2, pp. 216-224, 2006. DOI: 10.1109/TMECH.2006.871897

[198] D. Stoianovici, A. Patriciu, D. Petrisor, D. Mazilu, and L. Kavoussi, "A new type of motor: Pneumatic step motor," IEEE/ASME Transactions on Mechatronics, vol. 12, no. 1, pp. 98-106, 2007. DOI: 10.1109/TMECH.2006.886258

[199] "Micronor MR338 MR safe abslolute rotary encoder." (), [Online]. Available: https: $/ /$ micronor.com/product/mr338 (visited on 11/23/2021).

[200] "Micronor MR303 MR safe linear encoder." (), [Online]. Available: https://micronor com/product/mr303/ (visited on 10/14/2021).

[201] B. Jarrahi, J. Wanek, U. Mehnert, and S. Kollias, "An fMRI-compatible multiconfigurable handheld response system using an intensity-modulated fiber-optic sensor," Proceedings of the Annual International Conference of the IEEE Engineering in Medicine and Biology Society, EMBS, pp. 6349-6352, 2013. DOI: 10.1109/EMBC.2013. 6611006

[202] Y.-s. Kwon and W.-j. Kim, "Development of a New High-Resolution Angle-Sensing Mechanism Using an RGB Sensor," IEEE/ASME Transactions on Mechatronics, vol. 19, no. 5, pp. 1707-1715, Oct. 2014. DOI: 10.1109/TMECH.2013.2293571

[203] A. C. NELSON, "Precision Position Control Using an Rgb Sensor and Linearized Output Variable-Intensity Color Array," Ph.D. dissertation, Texas A\&M University, 2017, p. 96.

[204] P. L. M. Heydemann, "Determination and correction of quadrature fringe measurement errors in interferometers," Applied Optics, vol. 20, no. 19, p. 3382, Oct. 1981. DOI: 10.1364/A0.20.003382

[205] M. Van Kuijk, "Auto calibration of incremental analog quadrature encoders," Master's thesis, University of Eindhoven, 2009.

[206] P. Polygerinos, L. D. Seneviratne, and K. Althoefer, "Modeling of light intensitymodulated fiber-optic displacement sensors," IEEE Transactions on Instrumentation and Measurement, vol. 60, no. 4, pp. 1408-1415, 2011. DOI: 10.1109/TIM.2010.2085270 
[207] J. Peirs, J. Clijnen, D. Reynaerts, H. V. Brussel, P. Herijgers, B. Corteville, and S. Boone, "A micro optical force sensor for force feedback during minimally invasive robotic surgery," Sensors and Actuators A: Physical, vol. 115, no. 2-3, pp. 447-455, Sep. 2004. DOI: $10.1016 /$ j.sna.2004.04.057.

[208] W. Zhu, S. Salcudean, S. Bachmann, and P. Abolmaesumi, "Motion/force/image control of a diagnostic ultrasound robot," in Proceedings 2000 ICRA. Millennium Conference. IEEE International Conference on Robotics and Automation. Symposia Proceedings (Cat. No.00CH37065), vol. 2, IEEE, 2000, pp. 1580-1585, ISBN: 0-7803-5886-4. DOI: 10.1109/ROBOT.2000.844822

[209] F. Conti, J. Park, and O. Khatib, "Interface Design and Control Strategies for a Robot Assisted Ultrasonic Examination System," 2014.

[210] A. Krupa, "Automatic calibration of a robotized 3D ultrasound imaging system by visual servoing," in Proceedings 2006 IEEE International Conference on Robotics and Automation, 2006. ICRA 2006., IEEE, 2006, pp. 4136-4141, ISBN: 0-7803-9505-0. DOI: 10.1109/ROBOT. 2006.1642338

[211] R. Finocchi, F. Aalamifar, T. Y. Fang, R. H. Taylor, and E. M. Boctor, "Co-robotic ultrasound imaging: a cooperative force control approach," in Medical Imaging 201\%: Image-Guided Procedures, Robotic Interventions, and Modeling, R. J. Webster and B. Fei, Eds., vol. 10135, Mar. 2017, p. 1013 510. DOI: 10.1117/12.2255271

[212] M. Akbari, J. Carriere, T. Meyer, R. Sloboda, S. Husain, N. Usmani, and M. Tavakoli, "Robotic Ultrasound Scanning With Real-Time Image-Based Force Adjustment: Quick Response for Enabling Physical Distancing During the COVID-19 Pandemic," Frontiers in Robotics and AI, vol. 8, no. March, Mar. 2021. DOI: 10.3389/frobt.2021.645424

[213] J. Lachner, F. Allmendinger, E. Hobert, N. Hogan, and S. Stramigioli, "Energy budgets for coordinate invariant robot control in physical human-robot interaction," International Journal of Robotics Research, pp. 1-18, 2021. DOI: 10.1177/02783649211011639

[214] A. Ahmad, M. C. Cavusoglu, and O. Bebek, "Calibration of 2D Ultrasound in 3D space for Robotic biopsies," Proceedings of the 17th International Conference on Advanced Robotics, ICAR 2015, pp. 40-46, 2015. DOI: 10.1109/ICAR.2015.7251431

[215] R. WOODS, "Validation of Registration Accuracy," in Handbook of Medical Image Processing and Analysis, Second Edi, Elsevier, 2009, pp. 569-575, ISBN: 9780123739049 DOI: 10.1016/B978-012373904-9.50043-X

[216] A. Felden, J. Vagner, A. Hinz, H. Fischer, S. O. R. Pfleiderer, J. R. Reichenbach, and W. A. Kaiser, "ROBITOM- ROBOT FOR BIOPSY AND THERAPY OF THE MAMMA," Biomedizinische Technik/Biomedical Engineering, vol. 47, no. s1a, pp. 2-5, 2002. DOI: $10.1515 /$ bmte.2002.47.s1a.2

[217] S. Bellini, "On a unique behavior of freshwater bacteria," Chinese Journal of Oceanology and Limnology, vol. 27, no. 1, pp. 3-5, Feb. 2009. DOI: 10.1007/s00343-009-0003-5

[218] R. P. Blakemore, D. Maratea, and R. S. Wolfe, "Isolation and pure culture of a freshwater magnetic spirillum in chemically defined medium," Journal of Bacteriology, vol. 140, no. 2, pp. 720-729, Nov. 1979. DOI: 10.1128/jb.140.2.720-729.1979

[219] C. T. Lefèvre, M. Bennet, L. Landau, P. Vach, D. Pignol, D. A. Bazylinski, R. B. Frankel, S. Klumpp, and D. Faivre, "Diversity of Magneto-Aerotactic Behaviors and Oxygen Sensing Mechanisms in Cultured Magnetotactic Bacteria," Biophysical Journal, vol. 107, no. 2, pp. 527-538, Jul. 2014. DOI: $10.1016 / \mathrm{j} . \mathrm{bpj} .2014 .05 .043$

[220] D. Sch $\tilde{A} 1 / 4$ ler, R. Uhl, and E. BÃ o uerlein, "A simple light scattering method to assay magnetism in Magnetospirillum gryphiswaldense," FEMS Microbiology Letters, vol. 132, no. 1-2, pp. 139-145, Oct. 1995. DOI: 10.1111/j.1574-6968.1995.tb07823.x 
[221] D. Faivre, A. Fischer, I. Garcia-Rubio, G. Mastrogiacomo, and A. U. Gehring, "Development of Cellular Magnetic Dipoles in Magnetotactic Bacteria," Biophysical Journal, vol. 99, no. 4, pp. 1268-1273, Aug. 2010. DOI: 10.1016/j.bpj.2010.05.034

[222] A. Fernández-Castané, H. Li, O. R. Thomas, and T. W. Overton, "Development of a simple intensified fermentation strategy for growth of Magnetospirillum gryphiswaldense MSR-1: Physiological responses to changing environmental conditions," New Biotechnology, vol. 46, pp. 22-30, Nov. 2018. DOI: $10.1016 / \mathrm{j} \cdot \mathrm{nbt.2018.05.1201.}$

[223] J. Yang, S. Li, X. Huang, T. Tang, W. Jiang, T. Zhang, and Y. Li, "A key time point for cell growth and magnetosome synthesis of Magnetospirillum gryphiswaldense based on real-time analysis of physiological factors," Frontiers in Microbiology, vol. 4, p. 210, 2013. DOI: $10.3389 / \mathrm{fmicb} .2013 .00210$

[224] T. Song, L. Zhao, and L.-F. Wu, "A Method for Quantitative Determination of the Number of Magnetosomes in Magnetotactic Bacteria by a Spectrophotometer," IEEE Transactions on Magnetics, vol. 50, no. 11, pp. 1-4, Nov. 2014. DOI: 10.1109/TMAG 2014.2323953 .

[225] C. T. Lefèvre, T. Song, J.-P. Yonnet, and L.-F. Wu, "Characterization of Bacterial Magnetotactic Behaviors by Using a Magnetospectrophotometry Assay," Applied and Environmental Microbiology, vol. 75, no. 12, pp. 3835-3841, Jun. 2009. DoI: 10.1128/ AEM.00165-09

[226] C.-Y. Chen, C.-F. Chen, Y. Yi, L.-J. Chen, L.-F. Wu, and T. Song, "Construction of a microrobot system using magnetotactic bacteria for the separation of Staphylococcus aureus," Biomedical Microdevices, vol. 16, no. 5, pp. 761-770, Oct. 2014. DoI: 10.1007/ s10544-014-9880-2

[227] M. Bennet, D. Gur, J. Yoon, Y. Park, and D. Faivre, "A Bacteria-Based Remotely Tunable Photonic Device," Advanced Optical Materials, vol. 5, no. 1, p. 1600617 , Jan. 2017. DOI: 10.1002 /adom. 201600617

[228] E. Katzmann, M. Eibauer, W. Lin, Y. Pan, J. M. Plitzko, and D. Schüler, "Analysis of Magnetosome Chains in Magnetotactic Bacteria by Magnetic Measurements and Automated Image Analysis of Electron Micrographs," Applied and Environmental Microbiology, vol. 79, no. 24, pp. 7755-7762, Dec. 2013. DOI: 10.1128/AEM.02143-13

[229] J. A. Myers, B. S. Curtis, and W. R. Curtis, "Improving accuracy of cell and chromophore concentration measurements using optical density," BMC Biophysics, vol. 6, no. 1, p. 4, Dec. 2013. DOI: 10.1186/2046-1682-6-4

[230] L. Zhao, D. Wu, L.-F. Wu, and T. Song, "A simple and accurate method for quantification of magnetosomes in magnetotactic bacteria by common spectrophotometer," Journal of Biochemical and Biophysical Methods, vol. 70, no. 3, pp. 377-383, Apr. 2007. DOI: $10.1016 / \mathrm{j} \cdot \mathrm{jbbm} .2006 .08 .010$

[231] C. Lang and D. Schüler, "Expression of Green Fluorescent Protein Fused to Magnetosome Proteins in Microaerophilic Magnetotactic Bacteria," Applied and Environmental Microbiology, vol. 74, no. 15, pp. 4944-4953, Aug. 2008. DOI: 10.1128/AEM.00231-08

[232] D. Schüler and E. Baeuerlein, "Dynamics of Iron Uptake and Fe 3 O 4 Biomineralization during Aerobic and Microaerobic Growth of Magnetospirillum gryphiswaldense," Journal of Bacteriology, vol. 180, no. 1, pp. 159-162, Jan. 1998. DOI: 10.1128/JB.180.1.159162.1998

[233] U. Heyen and D. Schüler, "Growth and magnetosome formation by microaerophilic Magnetospirillum strains in an oxygen-controlled fermentor," Applied Microbiology and Biotechnology, vol. 61, no. 5-6, pp. 536-544, Jun. 2003. DOI: 10.1007/s00253-002-1219$\mathrm{X}$ 
[234] M. Pichel, T. Hageman, I. Khalil, A. Manz, and L. Abelmann, "Magnetic response of Magnetospirillum gryphiswaldense observed inside a microfluidic channel," Journal of Magnetism and Magnetic Materials, vol. 460, pp. 340-353, Aug. 2018. DOI: $10.1016 / \mathrm{j}$ jmmm.2018.04.004

[235] C. Zahn, S. Keller, M. Toro-Nahuelpan, P. Dorscht, W. Gross, M. Laumann, S. Gekle, W. Zimmermann, D. Schüler, and H. Kress, "Measurement of the magnetic moment of single Magnetospirillum gryphiswaldense cells by magnetic tweezers," Scientific Reports, vol. 7, no. 1, p. 3558, Dec. 2017. DOI: 10.1038/s41598-017-03756-z

[236] D. M. S. Esquivel and H. G. P. Lins De Barros, "Motion of Magnetotactic Microorganisms," Journal of Experimental Biology, vol. 121, no. 1, pp. 153-163, Mar. 1986. DOI: 10.1242/jeb.121.1.153.

[237] C. Kittel, Introduction to Solid State Physics (3rd ed.) New York; London: Wiley, 1966.

[238] J. Simpson, J. Lane, C. Immer, and R. Youngquist, "Simple analytic expressions for the magnetic field of a circular current loop," NASA technical documents, 2001.

[239] F. Popp, J. P. Armitage, and D. Schüler, "Polarity of bacterial magnetotaxis is controlled by aerotaxis through a common sensory pathway," Nature Communications, vol. 5 , no. 1, p. 5398, Dec. 2014. DOI: 10.1038/ncomms6398

[240] S. S. Staniland, C. Moisescu, and L. G. Benning, "Cell division in magnetotactic bacteria splits magnetosome chain in half," Journal of Basic Microbiology, vol. 50, no. 4, pp. 392-396, May 2010. DOI: 10.1002/jobm.200900408

[241] C.-D. Yang, H. Takeyama, T. Tanaka, A. Hasegawa, and T. Matsunaga, "Synthesis of Bacterial Magnetic Particles During Cell Cycle of Magnetospirillum magneticum AMB-1," Applied Biochemistry and Biotechnology, vol. 91-93, no. 1-9, pp. 155-160, 2001. DOI: 10.1385/ABAB:91-93:1-9:155

[242] D. A. Bazylinski, T. J. Williams, C. T. Lefèvre, R. J. Berg, C. L. Zhang, S. S. Bowser, A. J. Dean, and T. J. Beveridge, "Magnetococcus marinus gen. nov., sp. nov., a marine, magnetotactic bacterium that represents a novel lineage (Magnetococcaceae fam. nov., Magnetococcales ord. nov.) at the base of the Alphaproteobacteria," International Journal of Systematic and Evolutionary Microbiology, vol. 63, no. Pt_3, pp. 801-808, Mar. 2013. DOI: $10.1099 /$ ijs.0.038927-0

[243] C. T. Lefèvre, P. A. Howse, M. L. Schmidt, M. Sabaty, N. Menguy, G. W. Luther, and D. A. Bazylinski, "Growth of magnetotactic sulfate-reducing bacteria in oxygen concentration gradient medium," Environmental Microbiology Reports, vol. 8, no. 6, pp. 1003-1015, Dec. 2016. DOI: 10.1111/1758-2229.12479

[244] S.-H. Song, J. Yoon, Y. Jeong, Y.-G. Jung, L. Abelmann, and W. Park, "Quantifying and dispensing of magnetic particles in a self-assembled magnetic particle array," Journal of Magnetism and Magnetic Materials, vol. 539, no. May, p. 168341, Dec. 2021. DOI: $10.1016 / \mathrm{j} \cdot \mathrm{jmmm} .2021 .168341$

[245] T. L\{\"o\}thman, Per A. Hageman, J. Hendrix, H. H. Keizer, H. Van Wolferen, K. Ma, M. M. Van de Loosdrecht, B. Ten Haken, T. Bolhuis, and L. Abelmann, "Response of suspensions of microfabricated magnetic discs to time varying fields," in 8th International Workshop on Magnetic Particle Imaging, 2018.

[246] Y. Gao, A. Van Reenen, M. A. Hulsen, A. M. De Jong, M. W. Prins, and J. M. Den Toonder, "Disaggregation of microparticle clusters by induced magnetic dipole-dipole repulsion near a surface," Lab on a Chip, vol. 13, no. 7, pp. 1394-1401, 2013. DOI: 10.1039/c3lc41229f 